\title{
Bildungsevaluierung
}

\section{Ländliche Entwicklung LE 07-13}

\section{Endbericht}

Eine Arbeit im Auftrag des Bundesministeriums

für Land- und Forstwirtschaft, Umwelt und Wasserwirtschaft

24. Oktober 2013

ao Univ.Prof. DI Dr. Christoph Mandl

Mag. Theresa Kuttner, B.A.

MIT UNTERSTÜTZUNG VON BUND, LÄNDERN UND EUROPÄISCHER UNION

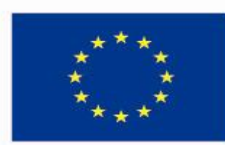


Endbericht Bildungsevaluierung Ländliche Entwicklung LE 07-13 


\section{INHALT}

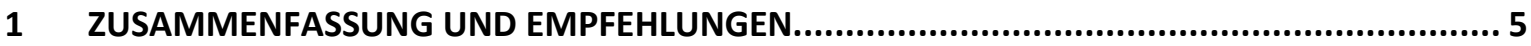

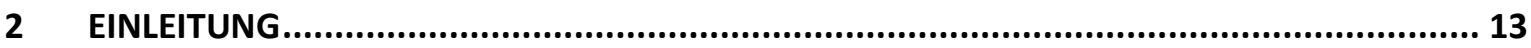

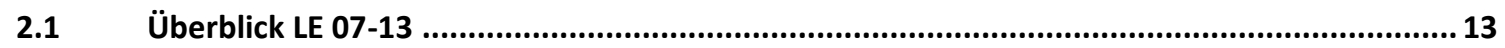

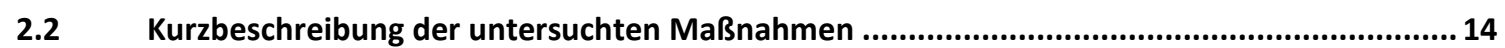

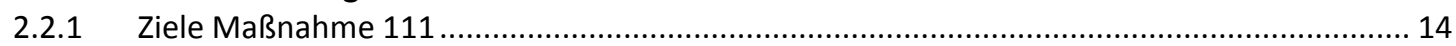

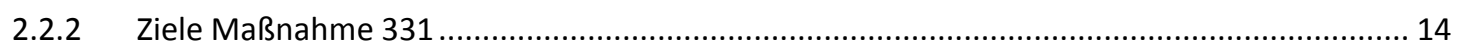

2.2.3 Ziele Agrar-Umwelt und Naturschutz / Agrarumweltmaßnahmen (M 214) ........................... 14

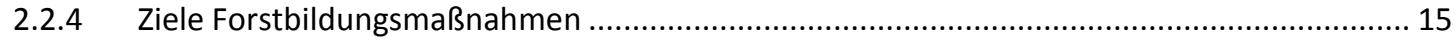

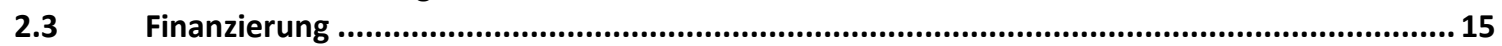

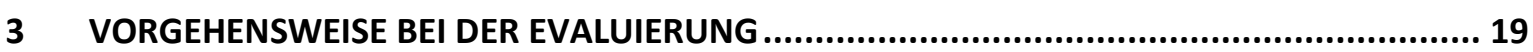

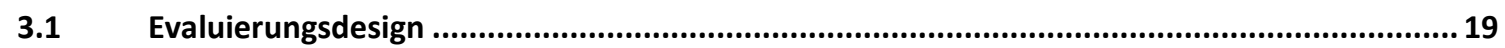

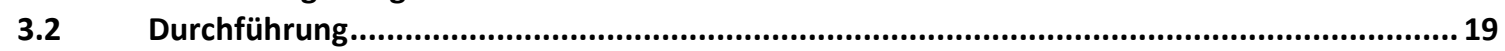

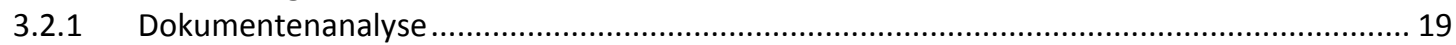

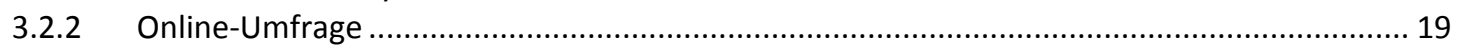

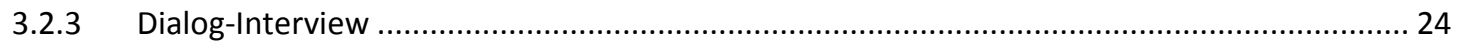

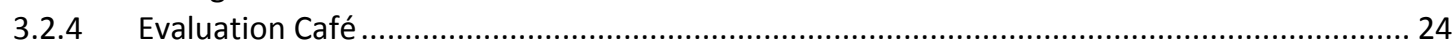

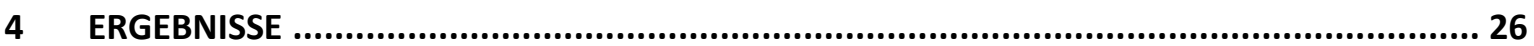

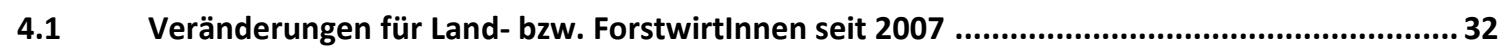

4.2 Wettbewerbsfähigkeit in der Land-, Ernährungs- oder Forstwirtschaft ....................................36

4.3 Nachhaltiges Flächenmanagement und nachhaltiges Management natürlicher Ressourcen .. 38

4.4 Bedarf an Bildungsmaßnahmen und Kohärenz mit anderen Maßnahmen des Programms .... 46

4.5 Verbesserung des Humanpotenzials ........................................................................................62

4.6 Diversifizierung in nicht-agrarische Bereiche ........................................................................67

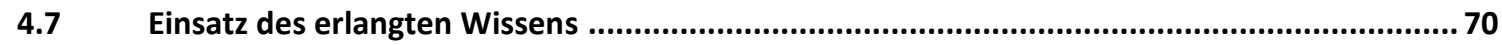

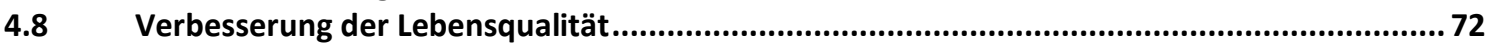

4.9 Regionale Entwicklung, Wissenstransfer und innovative Ideen ...............................................74

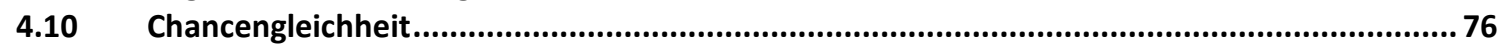

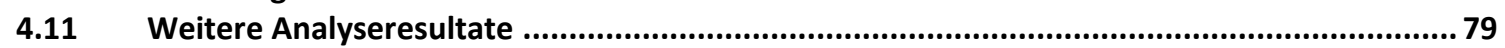

4.11.1 Seminarbäuerinnen, Schule-am-Bauernhof Betriebe, WaldpädagogInnen ......................... 79

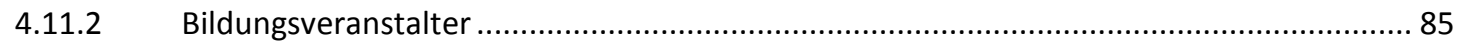

4.11.3 TeilnehmerInnen an Kursen der LE aus anderen Branchen ............................................ 97

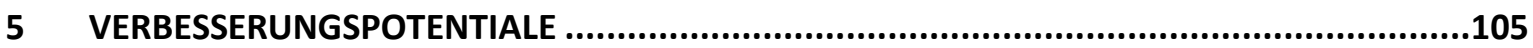

5.1 Prioritäten, Ziele und Schwerpunktsetzungen für LE 14-20 Priorität I ................................... 105

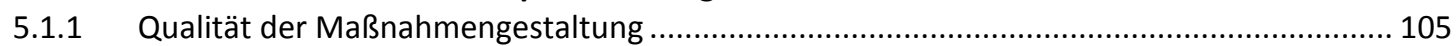

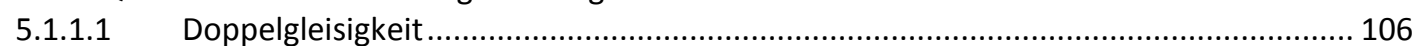

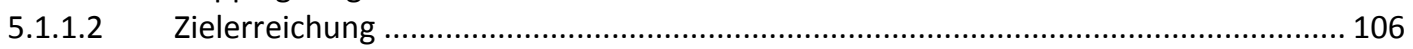

5.1.1.3 Verhältnismäßigkeit zwischen Kosten und Wirkungen ................................................ 106

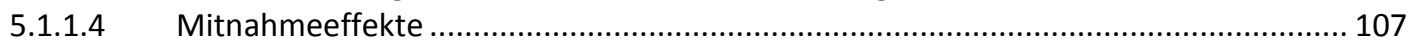

5.1.1.5 Bundesländerübergreifende Bildungsprojekte ...................................................... 107

5.1.1.6 Erreichung der gewünschten Zielgruppen ............................................................ 108

5.1.1.7 Genderspezifische Gestaltung der Bildungsmaßnahmen .......................................... 108

5.1.1.8 Beratungs- und Veranstaltungs-Förderungslandschaft .............................................. 109

5.1.1.9 Verbreitung wissenschaftlicher Erkenntnisse ....................................................... 111

5.1.1.10 Austausch und Vergleich mit anderen EU-Ländern .................................................. 112

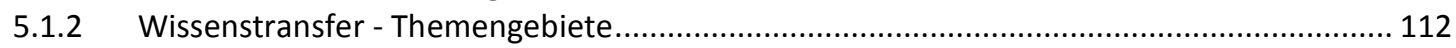

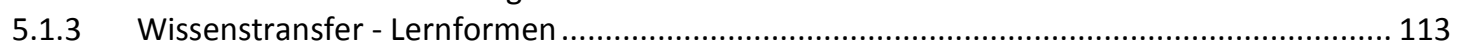

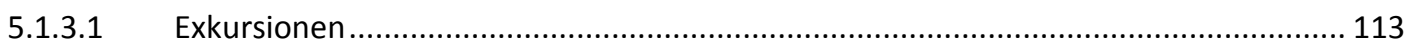

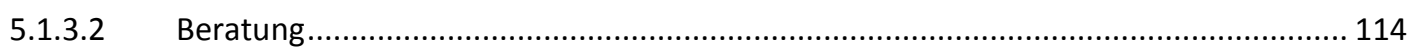

5.2 Strukturierung der Datenbasis für Auswertungen und Ergebnisse ....................................... 115

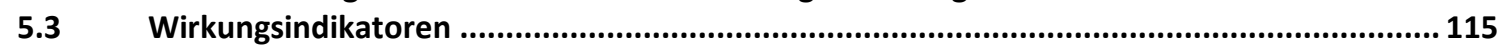


ANHÄNGE. 117

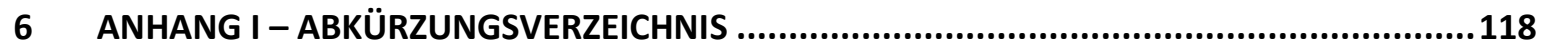

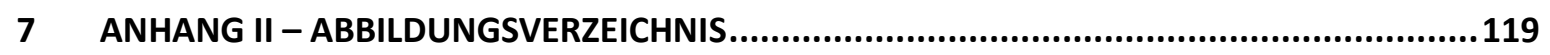

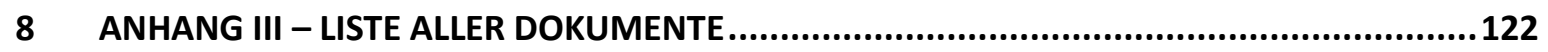




\section{ZUSAMMENFASSUNG UND EMPFEHLUNGEN}

Die ländlichen Gebiete der EU belaufen sich auf rund $91 \%$ der Gesamtfläche der Mitgliedstaaten (siehe auch http://ec.europa.eu/agriculture/rurdev/index_de.htm). Zur Verfolgung der gesetzten Ziele bzgl. Wachstum und Beschäftigung (Lissabon-Strategie) sowie nachhaltige Entwicklung (Strategie von Göteborg) in den ländlichen Räumen, setzt die EU auf eine gemeinschaftliche Politik zur Entwicklung des ländlichen Raums.

Für die Periode 2007 bis 2013 wurde aus der VO (EG) Nr. 1698/2005 das österreichweit gültige Programm für die Entwicklung des Ländlichen Raums abgeleitet. Das mehrere Maßnahmen umfassende Förderprogramm wurde dabei auf die drei, seitens der EU festgelegten Ziele für die Periode 2007 bis 2013 ausgerichtet.

Die Umsetzung des Programms zur Ländlichen Entwicklung unterliegt laut EU-Verordnung laufenden Monitoring- und Evaluierungsprozessen mit expliziten Vorgaben. In Österreich wird diese Aufgabe von der Bundesanstalt für Agrarwirtschaft (AWI) übernommen. Zusätzlich zu der laufenden Evaluierung durch das AWI wurde die vorliegende Studie / Evaluierung in Auftrag gegeben, welche die Wirkung der Bildungsmaßnahmen auf die Gesamtziele des Programms aufzeigen, sowie deren Umsetzung analysieren soll.

Die erwähnten Bildungsmaßnahmen betreffen konkret die Maßnahmen 111 (aus dem Schwerpunkt 1) und 331 (aus dem Schwerpunkt 3) des Programms LE07-13. Ihre Inhalte Ausbildung, Fortbildung, Weiterbildung und Information stellen wichtige Faktoren bei der betrieblichen Existenzsicherung, bei der Diversifizierung für zusätzliche Einkommensquellen und beim Verständnis für Nachhaltigkeit und regionale Entwicklung dar und sind somit wesentliche Bestandteile des Ländlichen Entwicklungsprogramms. Zusätzlich wurde auch auf die Bildungsmaßnahmen aus dem Forst-Bereich sowie aus dem Bereich Agrar-Umwelt und Naturschutz eingegangen.

Der Endbericht zur durchgeführten Studie analysiert zunächst die Wirkungen der Bildungsmaßnahmen, abschließend werden identifizierte Verbesserungsmöglichkeiten ausgearbeitet. Es werden Empfehlungen für die zukünftige Ausgestaltung und Durchführung der Maßnahmen abgeleitet und Indikatoren für die weitere Wirkungsevaluierung der Bildungsmaßnahmen festgelegt.

Insgesamt wurden in den Maßnahmen M 111 und M 331 zwischen in den Jahren 2007-2012 $€ 75.484 .932,30$ an Fördergeldern ausbezahlt. Von den 37 geförderten Veranstaltern erhielten 5, also $13,5 \%$, rund $61 \%$ der gesamten Veranstalterförderung. Dabei wird allerdings jedes LFI getrennt betrachtet. Wenn man die $10 \mathrm{LFIs}$ als eine zusammengehörige Organisation ansieht, dann erhielt diese eine Organisation, also 3,6 \% der 28 geförderten Veranstalter, $67 \%$ der gesamten Veranstalterförderung (Details hierzu siehe Kapitel 2.3). 


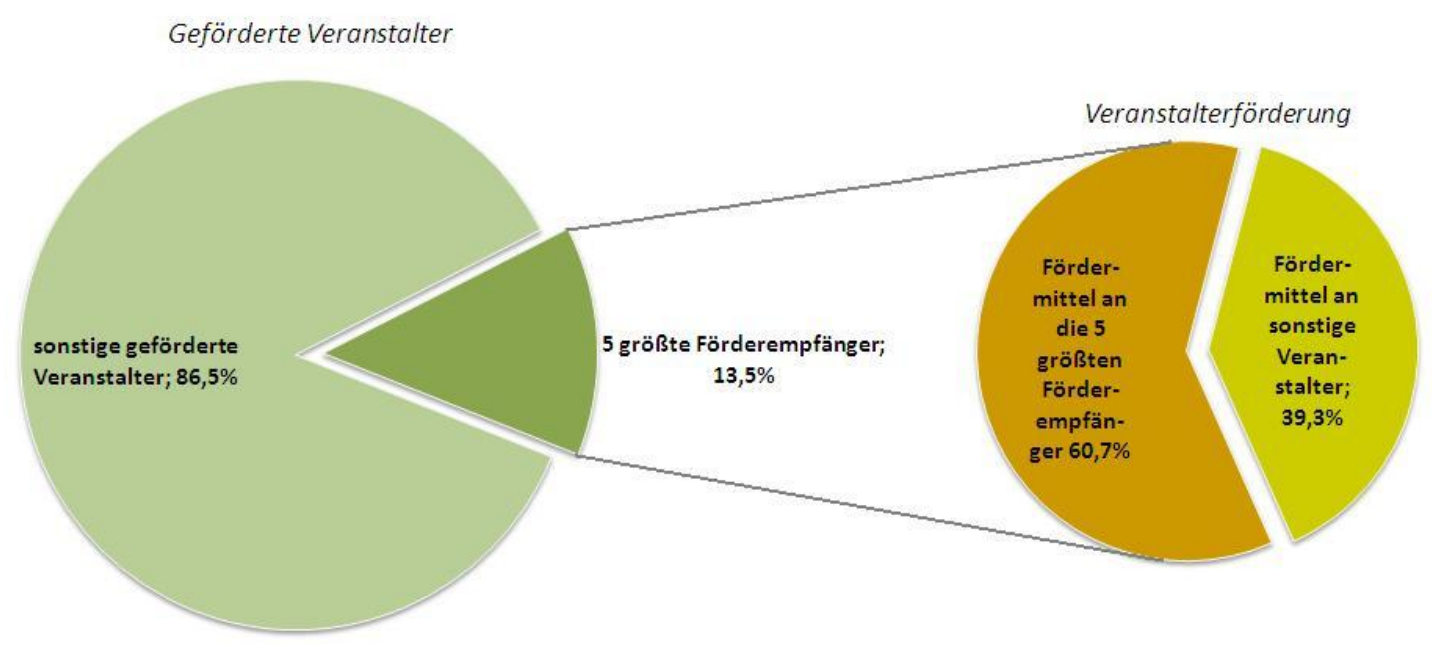

Abbildung II: Verteilung der Veranstalterförderung (M $111+$ M 331) - LFIs zusammengefasst

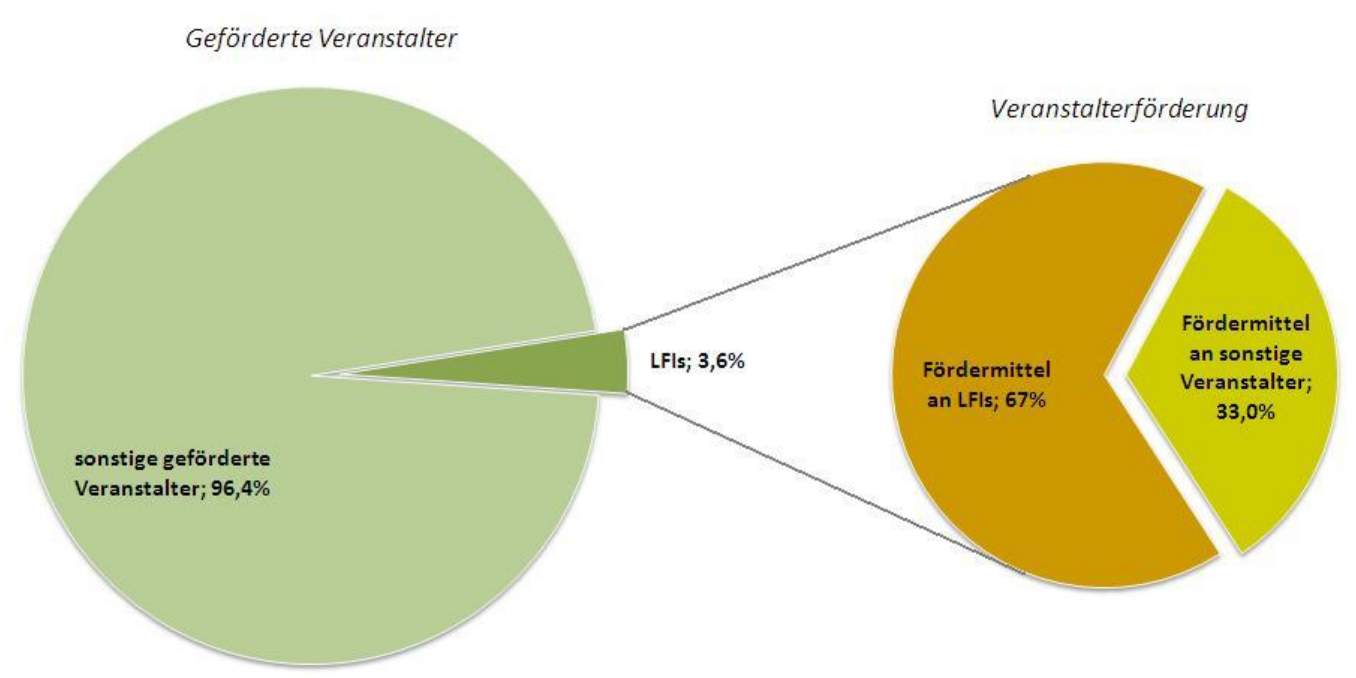

Die folgende Abbildung III zeigt die Antworten jener Personen, die seit 2007 an Bildungsangeboten teilgenommen haben im Vergleich zu jenen, die angaben, an keinen Bildungsangeboten teilgenommen zu haben. Dabei lassen sich merkbare Unterschiede feststellen. Jedoch ist auch festzuhalten, dass die Gruppe der Land- bzw. ForstwirtInnen, die keine Bildungsangebote besucht haben im Fragebogen weitaus geringer vertreten war $(\mathrm{N}=136)$, als die Vergleichsgruppe, die die Bildungsangebote der LE in Anspruch genommen hat $(\mathrm{N}=1030)$, wodurch sich eine gewisse Unschärfe ergibt.

Abbildung III ist somit ein zentrales Ergebnis dieser Studie, da ein wechselseitig verstärkender Zusammenhang zwischen dem Besuch von Bildungsmaßnahmen und der Einschätzung bezüglich der 11 zu Auswahl stehenden Kategorien empirisch feststellbar ist. Dies bedeutet, wie bei allen statistischen Korrelationen, nicht, dass eine Ursache-Wirkungs-Beziehung in die eine und/oder andere Richtung besteht, aber es bedeutet doch, dass alle jene Land- bzw. ForstwirtInnen, welche 
seit 2007 Bildungsmaßnahmen besucht haben, eine deutlich höhere Einschätzung bezüglich der positiven Veränderungen der 11 Kategorien haben als jene Land- bzw. ForstwirtInnen, die keine Bildungsangebote besucht haben, wobei der stärkste Zusammenhang in der Kategorie Wertschöpfung und der geringste Zusammenhang in der Kategorie Erzeugung und Nutzung erneuerbarer Energie festzustellen ist.

Abbildung III: Veränderungen für Land- bzw. ForstwirtInnen seit 2007 - nach Teilnahme

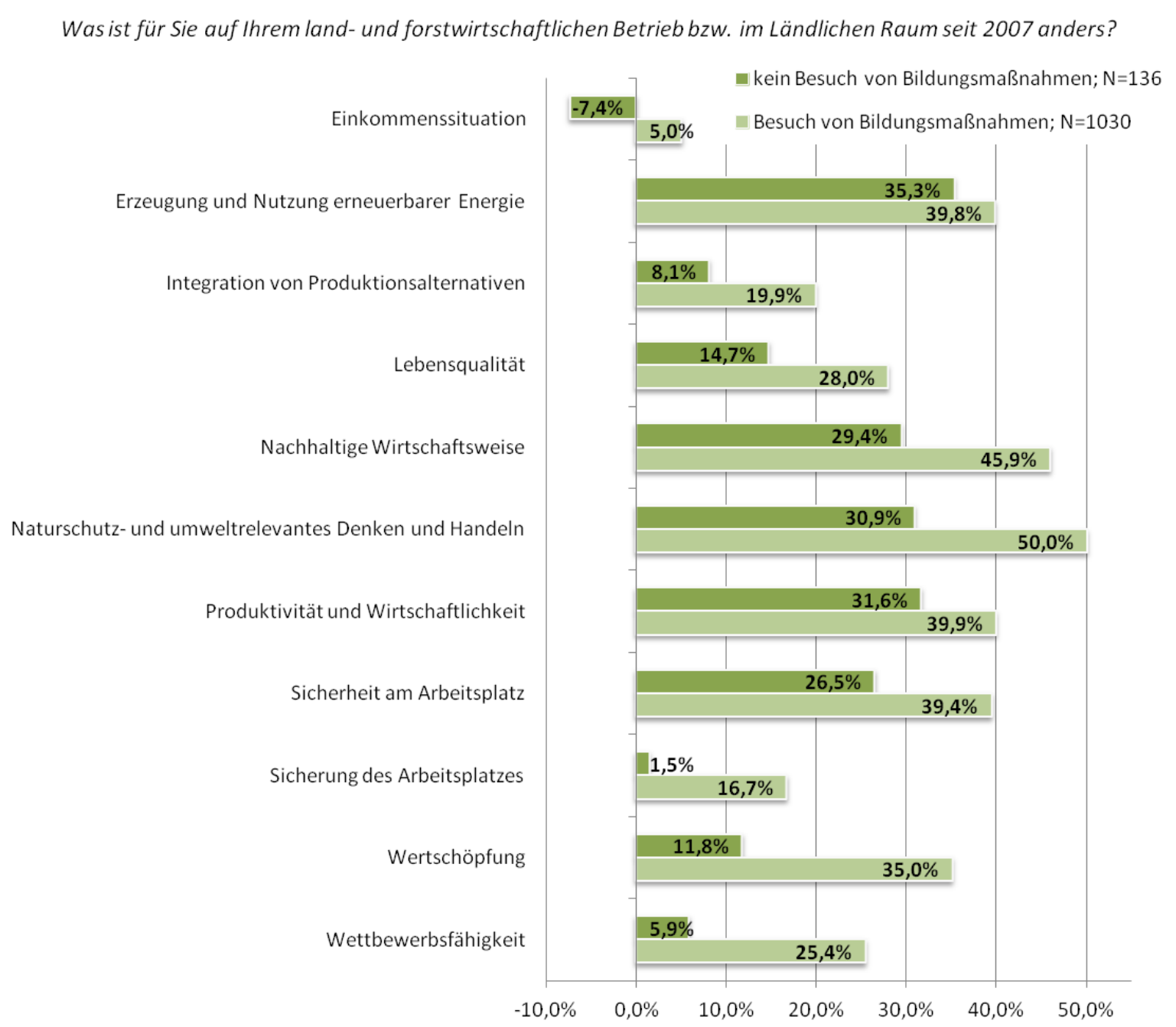

Wie sehr die Bildungsangebote der LE zur Verbesserung des Humanpotenzials beigetragen haben wird nachfolgend anhand der Auswertung der Online-Befragung bzgl. des Nutzens dargestellt. Diese zentrale Frage aus dem Fragebogen wird ergänzt durch eine vergleichende Auswertung zur Bewertung der in den Kursen zum Einsatz gekommenen Lernformen.

\section{Bildungsmaßnahmen M 111 \& M 331}

Im Vergleich zu den Antworten aus den Gruppen Agrar-Umwelt und Naturschutz und Forstbildungsmaßnahmen stuften Personen, die Kurse aus M 111 und M 331 besucht haben den Nutzen etwas weniger positiv ein - in dieser Gruppe wurde die Kategorie "Nützlich“ häufiger gewählt, als in den beiden nachfolgend abgebildeten Gruppen. 
Abbildung IV: Empfundener Nutzen der Bildungsangebote - M 111 \& M 331

Wie nützlich fanden Sie die Bildungsangebote der LE für Ihre berufliche Weiterentwicklung insgesamt?

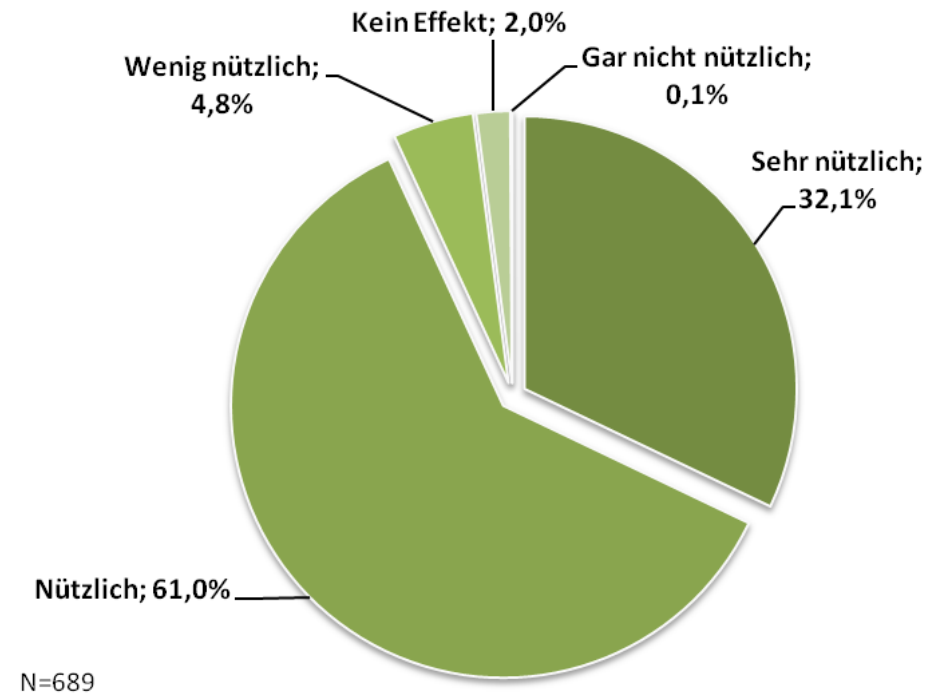

- Sehr nützlich - Hat mich entscheidend weiter gebracht.

- Nützlich - Ich habe neue / weiterführende Impulse für meine berufliche Tätigkeit erhalten.

Wenig nützlich - Geringe Verbesserung zu früher feststellbar.

Kein Effekt - Es ist für mich kein Unterschied zu früher feststellbar.

Gar nicht nützlich - Hat mich auf einen falschen beruflichen Weg gebracht.

\section{Agrar-Umwelt und Naturschutz}

Die Kategorie „gar nicht nützlich“ wurde in dieser Gruppe nicht ausgewählt.

Abbildung V: Empfundener Nutzen der Bildungsangebote - Agrar-Umwelt und Naturschutz

Wie nützlich fanden Sie die Bildungsangebote der LE für Ihre berufliche Weiterentwicklung insgesamt?

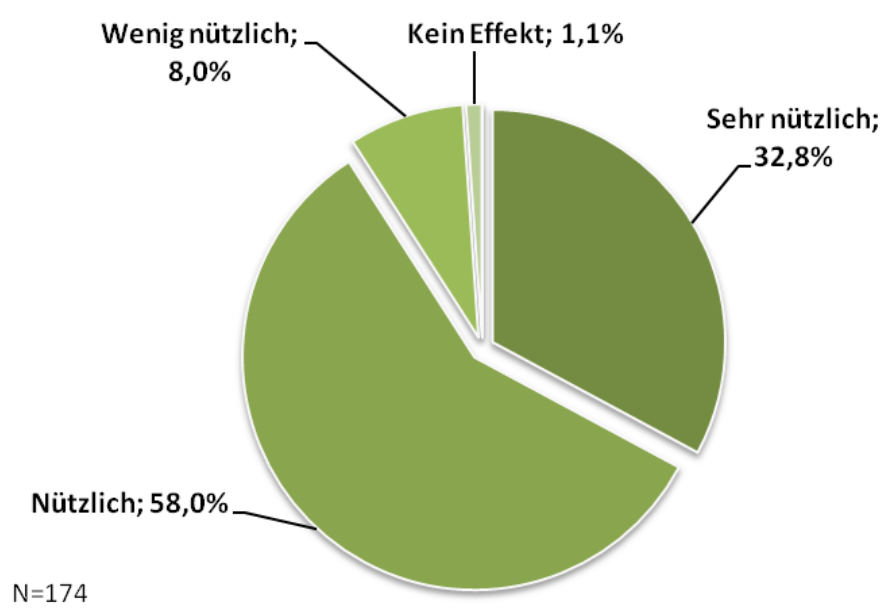
- Sehr nützlich - Hat mich entscheidend weiter gebracht.

Nützlich - Ich habe neue / weiterführende Impulse für meine berufliche Tätigkeit erhalten.

- Wenig nützlich - Geringe Verbesserung zu früher feststellbar.

Kein Effekt - Es ist für mich kein Unterschied zu früher feststellbar.

Ear nicht nützlich - Hat mich auf einen falschen beruflichen Weg gebracht.

\section{Forstbildungsmaßnahmen}

In der Gruppe der Personen, die Bildungsmaßnahmen aus dem forstlichen Bereich besuchten ist der Anteil derer, die diese Bildungsangebote als sehr nützlich empfinden deutlich größer, als bei den zuvor beschriebenen Gruppen. Die Kategorie "gar nicht nützlich“ wurde in dieser Gruppe nicht ausgewählt. 
Abbildung VI: Empfundener Nutzen der Bildungsangebote - Forstbildungsmaßnahmen

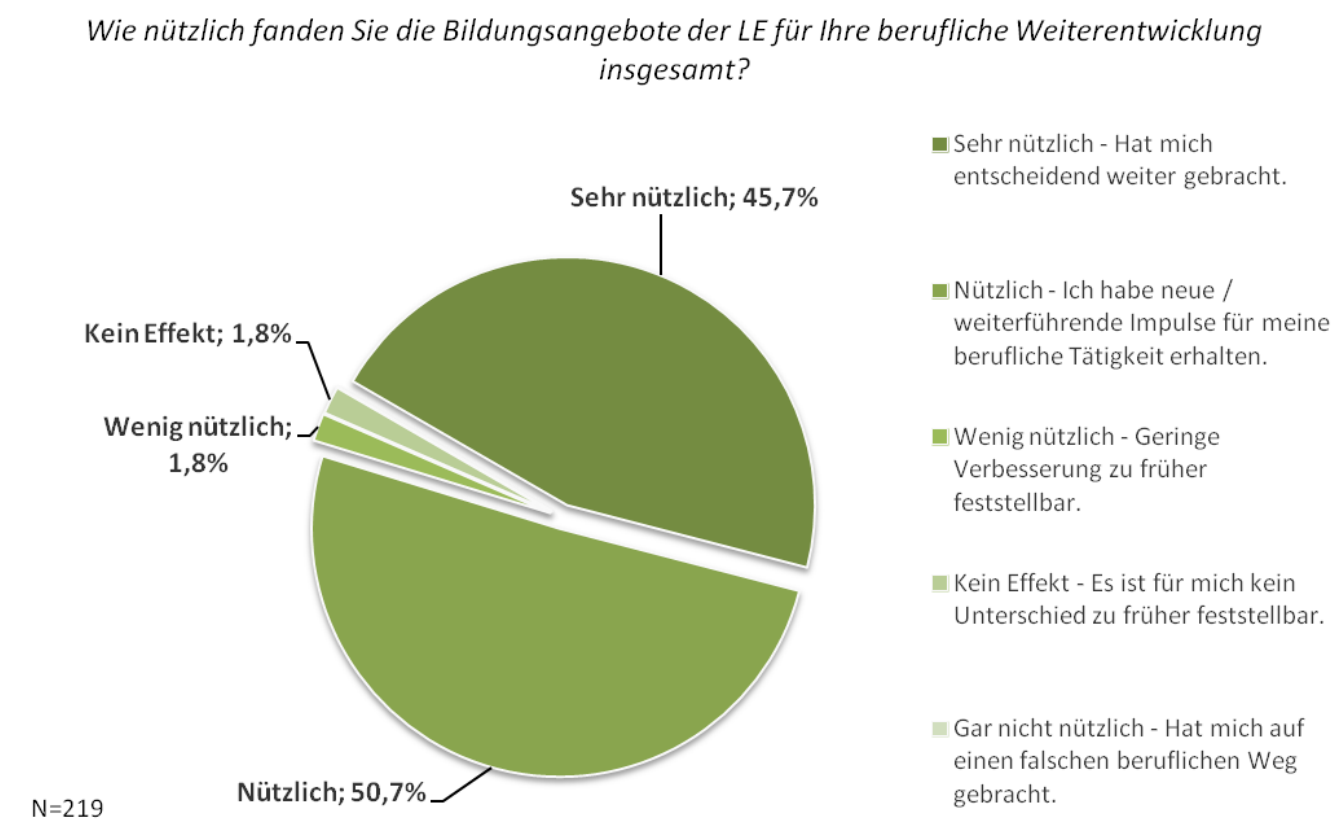

Sehr unterschiedlich sind die Gründe, warum auf eine Teilnahme an Bildungsangeboten der LE verzichtet wurde. Bei Frauen spielt dabei der Faktor Zeit eine wesentlich größere Rolle, als bei den Männern, die den Fragebogen beantworteten. Männer hingegen sehen sich bereits als ausreichend (aus-) gebildet und können keinen Nutzen für die berufliche Tätigkeit erkennen. Hoch ist bei beiden auch der Anteil jener, die angaben, keine Informationen zu den Bildungsangeboten erhalten zu haben. Der Preis für die Kurse und Veranstaltungen spielt sowohl bei den Frauen als auch bei den Männern eine eher unwichtigere Rolle.

Abbildung VII: Chancengleichheit - Gründe für Nicht-Teilnahme

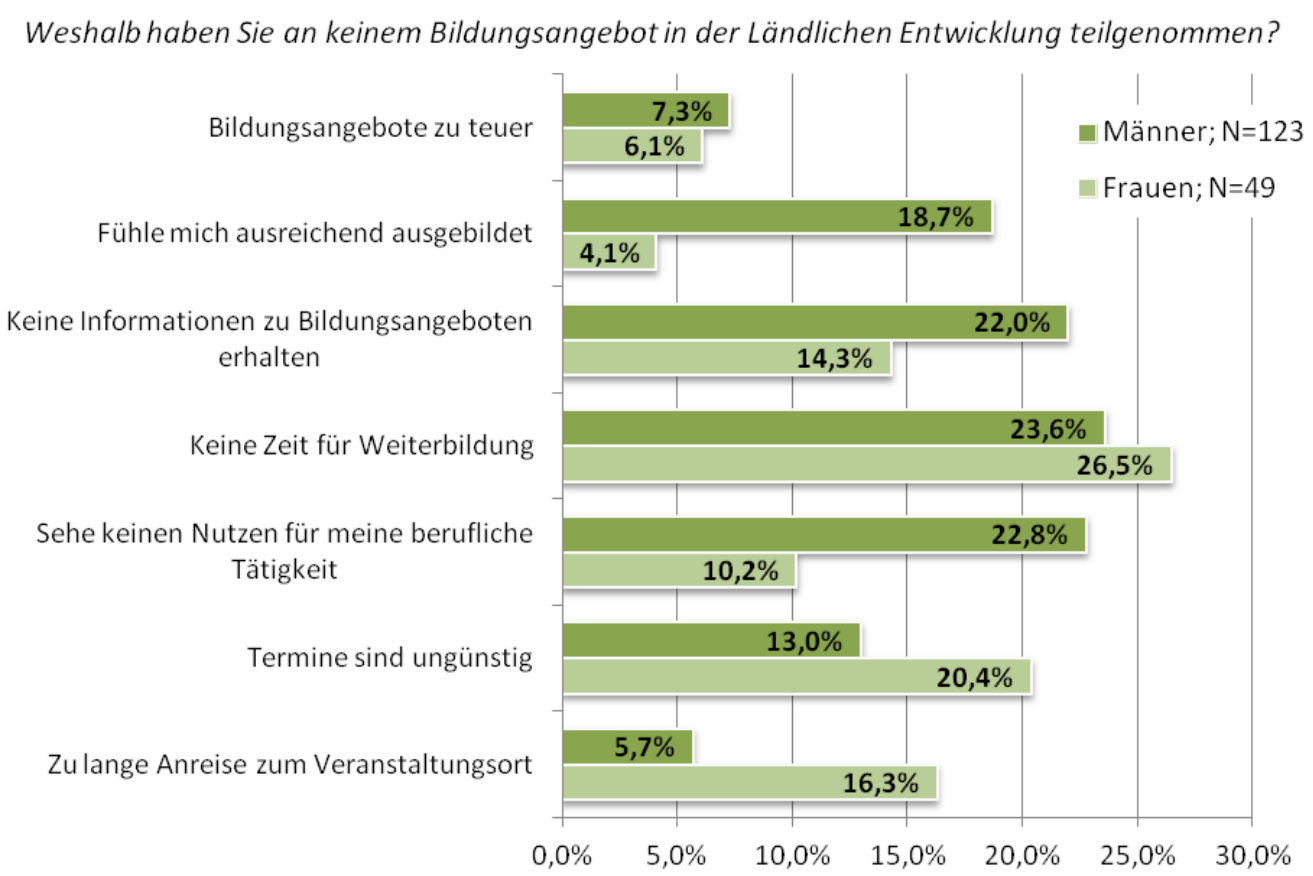




\section{Verbesserungspotenziale (Details hierzu siehe Kapitel 5)}

\section{Verhältnismäßigkeit zwischen Kosten und Wirkungen}

Die Kosten der Förderungsabwicklung sind aus Sicht der geförderten Veranstalter in Relation zum Förderungsbetrag hoch und insbesondere auch höher als in der vorangehenden Periode der LE. Da der Abrechnungsaufwand bei kleineren Förderbeträgen nicht entsprechend niedriger ist als bei großen Förderbeträgen, sind bei kleinen Förderbeträgen, insbesondere auch aber nicht nur bei TeilnehmerInnen-Förderungen die Kosten der Förderungsabwicklung besonders hoch. Eine Vereinfachung der Abrechnungsformalitäten mit dem Ziel, die Kosten der Förderungsabwicklung deutlich zu senken, wird daher empfohlen.

\section{Mitnahmeeffekte}

Der Mitnahmeeffekt bezeichnet die Inanspruchnahme finanzieller Förderungen als Belohnung für ein Verhalten, das auch ohne den zusätzlichen Anreiz stattgefunden hätte. Einiges deutet darauf hin, dass die Mitnahmeeffekte vor allem bei Förderung von "Vorbereitung, Durchführung und Nachbereitung von Berufsbildungs- und Informationsmaßnahmen" sowie bei TeilnehmerInnenFörderungen erheblich sind. Geringere Mitnahmeeffekte zeigen sind hingegen bei der „Erstellung von Bedarfsstudien oder Konzepten“ sowie bei der "Erstellung oder Ankauf von Unterlagen oder Hilfsmittel“. Deshalb wird empfohlen, die Förderung von „Vorbereitung, Durchführung und Nachbereitung von Berufsbildungs- und Informationsmaßnahmen" sowie TeilnehmerInnenFörderungen stark zu reduzieren bzw. auslaufen zu lassen und dafür die Förderung von „Erstellung von Konzepten" sowohl für Weiterbildungsmaßnahmen als auch für Beratungsmaßnahmen ebenso stark auszuweiten. Dies ist auch im Einklang mit der Ordnungspolitik der Europäischen Kommission primär die sogenannten vorwettbewerblichen (pre-competitive) Aktivitäten, wie Forschung, Entwicklung und Konzepterarbeitung, zu fördern und nicht die Erbringung von Dienstleistungen selbst, weil die Förderung der Erbringung von Dienstleistungen selbst stets eine marktverzerrende Wirkung hat zwischen jenen, deren Dienstleistungserbringung gefördert werden, und jenen, die eine solche Förderung nicht erhalten.

\section{Bundesländerübergreifende Bildungsprojekte}

Bundesländerübergreifende Bildungsprojekte nehmen eine wichtige Sonderstellung aus zwei Gründen ein: Zum Einen war die Förderung der „Erstellung von Bedarfsstudien oder Konzepten“ sowie der „Erstellung oder Ankauf von Unterlagen oder Hilfsmittel“ nach Aussagen in den DialogInterviews ausschließlich im Rahmen bundesländerübergreifender Bildungsprojekte möglich. Zum Anderen konnten Landwirtschaftskammer-ferne Veranstalter Förderungen nur im Rahmen bundesländerübergreifender Bildungsprojekte in Anspruch nehmen, da nur das BMLFUW den Landwirtschaftskammer-fernen Veranstaltern diese Möglichkeit bot, wohingegen in den Bundesländern selbst fast ausschließlich die LFIs Förderungen erhielten. Innovative Bildungsprojekte finden sich daher vorzugsweise in der Gruppe der bundesländerübergreifenden Bildungsprojekte, wohingegen die Bildungsprojekte in den Bundesländern eher durch die Förderung bestehender LFIAngebote charakterisiert sind, mit vermuteten hohen Mitnahmeeffekten.

\section{Erreichung der gewünschten Zielgruppen}

Bei den Zielgruppen fällt auf, dass bei der Online-Umfrage immerhin 23\% der Personen, welche an Kursen der Ländlichen Entwicklung teilgenommen haben, keine Land- oder ForstwirtInnen 
sind. Umgekehrt gaben bei der Online-Umfrage 13\% der Land- oder ForstwirtInnen an, seit 2007 keine Kurse der Ländlichen Entwicklung besucht zu haben. Gemäß Abb. 112 „Besuchte Kurse Vergleich nach Branche" zeigt sich indes, dass diese $23 \%$ der Personen vor allem Bildungsmaßnahmen M 331 und zu einem deutlich geringeren Teil Bildungsmaßnahmen M 111 in Anspruch genommen haben. Damit fällt die Erreichung der gewünschten Zielgruppen durchaus positiv aus. Trotzdem muss darauf hingewiesen werden, dass gemäß Abb. 112 auch Personen, die keine Landoder ForstwirtInnen sind, an Bildungsmaßnahmen M 111 teilgenommen haben. Darüber hinaus ist anzumerken, dass die Zielgruppen bei der Bildungsmaßnahme 331 nicht sehr präzise beschrieben sind. Um daher für die nächste Periode die Begünstigung von Personen, die nicht zur Zielgruppe gehören, zu vermeiden, bietet sich zur Umsetzung der Verordnung an, eine Förderungsabwicklung basierend auf dem Modell des Innovationsschecks, wie von der Österreichischen Forschungsförderungsgesellschaft (FFG) angewendet (Details siehe Kapitel 5.1.1.6), zu realisieren.

\section{Genderspezifische Gestaltung der Bildungsmaßnahmen}

Wie aus den Auswertungen (siehe auch Kapitel 4.10 insbes. Abbildung 79) hervorgeht, gibt es noch großes Potenzial, die Zielgruppe der Frauen für Bildungsangebote zu gewinnen. Denn während Männer sich zu einem großen Teil schon als genug ausgebildet / wissend einstufen, und daher eine Inanspruchnahme von Bildung als nicht attraktiv gesehen wird, sehen die befragten Frauen eher eine Wissenslücke. Als wichtiger, hinderlicher Faktor bzgl. Teilnahme an den diversen Angeboten wurde seitens der Frauen "Zeit" identifiziert. In Zukunft gilt es daher, besonders auf die Bedürfnisse von Frauen einzugehen. Die hohen Antwortzahlen in den Kategorien "Termine sind ungünstig“ und „Zu lange Anreise zum Veranstaltungsort" zeigen konkret Bereiche auf, wo rasch und unkompliziert reagiert werden kann, um mehr Frauen die Möglichkeit der Teilnahme zu bieten.

\section{Beratungs- und Veranstaltungs-Förderungslandschaft}

Eine wichtige Ursache für die vergleichsweise geringe Zuteilung von Fördermitteln an andere Veranstalter als Landwirtschaftskammern und LFIs dürfte sein, dass die Fördergeber als formale Voraussetzung den Nachweis eines zertifizierten Qualitätsmanagementsystems verlangen. So sinnvoll dies für große Industriebetriebe ist, so wenig bewährt sich ein solches zertifiziertes QM-System bei der Überprüfung, ob eine Organisation, welche Beratungs- oder Weiterbildungsleistungen anbietet, das entsprechende Wissen hat. Es wird daher empfohlen, bei der Auswahl von Projekten in LE 2020 das Formalkriterium "zertifiziertes QM-System" abzuschaffen und durch das Formalkriterium Gewerbeschein, Ingenieurkonsulent, Land- und Forstwirt bzw. Wissenschaftseinrichtung (Universitäten und Fachhochschulen) zu ersetzen. Weiters wird empfohlen, über entsprechende, regelmäßig stattfindende und stichtagsbezogene öffentliche Ausschreibungen zur Einreichung von Projekten aufzufordern und dann die, im Sinne der LE 2020-Ziele bestgeeignetsten Projekte durch eine ExpertInnen-Jury zur Förderung auszuwählen und die Ablehnung von Projekten so zu begründen, dass die Förderwerber davon lernen und es beim nächsten Mal besser machen können. Orientieren könnte sich diese Vorgehensweise bei der Auswahl förderbarer Projekte an der 2004 gegründeten Österreichischen Forschungsförderungsgesellschaft.

\section{Verbreitung wissenschaftlicher Erkenntnisse}

Beim Vergleich mit Programmen der LE anderer Europäischer Länder wie bspw. Schweden fällt auf, dass besonders in der Maßnahme 111 der Teilaspekt betreffend die Verbreitung wissenschaftlicher Erkenntnisse und innovativer Verfahren in der Umsetzung des Programms in den Hin- 
tergrund tritt. In Hinblick auf den, im Programm für die Periode 2014-2020 wichtigen neuen Schwerpunkt Wissenstransfer und Innovation sollte indes besonderes Augenmerk auf folgende Aspekte gelegt werden: Schaffung von "Meeting-Places“ zum Austausch von Informationen zwischen Wissenschaftlern und land- und forstwirtschaftlichen Betrieben; Erstellung einer Wissensdatenbank bzw. Webplattform, um den in der Land- und Forstwirtschaft tätigen Personen direkten Zugriff zu Ergebnissen aus der Forschung zu ermöglichen; insbesondere aber auch die Öffnung der Beratungs- und Veranstaltungs-Förderungslandschaft.

\section{Austausch und Vergleich mit anderen EU-Ländern}

Generell wird ein systematischer Austausch und Benchmarking sowohl auf der Ebene der Landund ForstwirtInnen, als auch auf der Ebene der Beratungs- und Weiterbildungsanbieter sowie auf der Ebene der Förderstellen mit anderen Ländern der EU empfohlen, denn Wissenstransfer und Innovation erfolgt umso effektiver, je größer der Wissenspool ist, aus dem geschöpft wird.

\section{Exkursionen}

Exkursionen wurden in der LE zuletzt nicht mehr gefördert. Im Vorschlag zur EU-Verordnung für die Programmperiode 2014-2020 heißt es dazu: „Der Wissenstransfer und die Informationsaktionen sollten nicht nur über herkömmliche Schulungen erfolgen, sondern den Bedürfnissen der ländlichen Akteure angepasst sein. Daher sollten Workshops, Coaching, Demonstrationstätigkeiten, Informationsaktionen, aber auch kurzzeitige Austausch und Besuchsprogramme für Landwirte unterstützt werden."

\section{Beratung}

Beratung und Weiterbildung sollten als zusammengehörig und einfach als zwei verschiedene Formen des Wissenstransfers gesehen werden. Darüber hinaus ist das Wissen, welches für eine moderne, innovative Land- und Forstwirtschaft erforderlich ist, bereits viel zu aufgefächert und diversifiziert, als dass dies bei den Landwirtschaftskammern alleine angesiedelt werden kann.

Deshalb wird empfohlen, dass die Förderung

- sowohl von Beratung als auch von Bildung gleich gestaltet wird,

- offen für alle Organisationen wird, welche zur Erbringung von Beratungs- oder BildungsLeistungen nach österreichischem Recht befugt bzw. konzessioniert sind, und

- der vorhandenen Marktdominanz bei Beratung und Bildung in der Land- und Forstwirtschaft entgegenwirkt. 


\section{EINLEITUNG}

\section{1 Überblick LE 07-13}

Die ländlichen Gebiete der EU belaufen sich auf rund 91\% der Gesamtfläche der Mitgliedstaaten (siehe auch http://ec.europa.eu/agriculture/rurdev/index_de.htm). Zur Verfolgung der gesetzten Ziele bzgl. Wachstum und Beschäftigung (Lissabon-Strategie) sowie nachhaltige Entwicklung (Strategie von Göteborg) in den ländlichen Räumen, setzt die EU auf eine gemeinschaftliche Politik zur Entwicklung des ländlichen Raums.

Für die Periode 2007 bis 2013 wurde aus der VO (EG) Nr. 1698/2005 das österreichweit gültige Programm für die Entwicklung des Ländlichen Raums abgeleitet. Das mehrere Maßnahmen umfassende Förderprogramm wurde dabei auf die drei, seitens der EU festgelegten Ziele für die Periode 2007 bis 2013 ausgerichtet. Die erwähnten Ziele aus der EU-Verordnung lauten:

- Steigerung der Wettbewerbsfähigkeit der Landwirtschaft und der Forstwirtschaft durch die Förderung der Umstrukturierung, der Entwicklung und der Innovation,

- Verbesserung der Umwelt und der Landschaft durch Förderung der Landbewirtschaftung,

- Steigerung der Lebensqualität im ländlichen Raum und Förderung der Diversifizierung der Wirtschaft.

Diese übergeordneten Ziele werden im laufenden Förderprogramm mittels der folgenden Schwerpunktsetzungen verfolgt:

Schwerpunkt 1 „Verbesserung der Wettbewerbsfähigkeit der Land- und Forstwirtschaft"

Schwerpunkt 2 "Verbesserung der Umwelt und der Landschaft"

Schwerpunkt 3 "Lebensqualität im ländlichen Raum und Diversifizierung der ländlichen Wirtschaft"

Schwerpunkt 4 „LEADER"

Die Umsetzung des Programms zur Ländlichen Entwicklung unterliegt laut EU-Verordnung laufenden Monitoring- und Evaluierungsprozessen mit expliziten Vorgaben. Zusätzlich wurde die vorliegende Studie / Evaluierung in Auftrag gegeben, um die Wirkung der Bildungsmaßnahmen auf die Gesamtziele des Programms aufzuzeigen, sowie deren Umsetzung zu analysieren.

Die erwähnten Bildungsmaßnahmen betreffen konkret die Maßnahmen 111 (aus dem Schwerpunkt 1) und 331 (aus dem Schwerpunkt 3) des Programms LE07-13. Ihre Inhalte Ausbildung, Fortbildung, Weiterbildung und Information stellen wichtige Faktoren bei der betrieblichen Existenzsicherung, bei der Diversifizierung für zusätzliche Einkommensquellen und beim Verständnis für Nachhaltigkeit und regionale Entwicklung dar und sind somit wesentliche Bestandteile des Ländlichen Entwicklungsprogramms. Zusätzlich wurde auch auf die Bildungsmaßnahmen aus dem Forst-Bereich sowie aus dem Bereich Agrar-Umwelt und Naturschutz eingegangen.

Der vorliegende Endbericht zur durchgeführten Studie analysiert zunächst die Wirkungen der Bildungsmaßnahmen, abschließend werden identifizierte Verbesserungsmöglichkeiten ausgearbeitet. Es werden Empfehlungen für die zukünftige Ausgestaltung und Durchführung der Maßnahmen abgeleitet und Indikatoren für die weitere Wirkungsevaluierung der Bildungsmaßnahmen festgelegt. 


\subsection{Kurzbeschreibung der untersuchten Maßnahmen}

In den nachstehenden Unterkapiteln werden jeweils die Ziele der im Rahmen dieser Evaluierung untersuchten Maßnahmen angeführt. Mittels der folgenden Fördergegenstände / förderbaren Aktivitäten wird die Erreichung dieser Ziele angestrebt:

1. Erstellung von Bedarfsstudien oder Konzepten für (Aus-) Bildungs- und Informationsmaßnahmen und (Aus-) Bildungs-und Informationsprodukte;

2. Erstellung oder Ankauf von Unterlagen oder Hilfsmitteln für den Einsatz bei (Aus-) Bildungs- und Informationsmaßnahmen

3. Vorbereitung, Durchführung und Nachbereitung von (Aus-) Bildungs- und Informationsmaßnahmen

\subsubsection{Ziele Maßnahme 111}

In der Maßnahme „Berufsbildung und Informationsmaßnahmen, einschließlich der Verbreitung wissenschaftlicher Erkenntnisse und innovativer Verfahren (M 111)" wird der Schwerpunkt 1 „Verbesserung der Wettbewerbsfähigkeit der Land- und Forstwirtschaft" herunter gebrochen auf die Zielsetzungen

- Erhöhung der Produktivität und Wirtschaftlichkeit

- Erhöhung der Wertschöpfung

- Steigerung von Produktionsalternativen und Erneuerbarer Energie

- Erhöhung der nachhaltigen Wirtschaftsweise

- Sicherung des Arbeitsplatzes

- Stärkung des naturschutz- und umweltrelevanten Denkens und Handelns.

\subsubsection{Ziele Maßnahme 331}

Für die Maßnahme "Ausbildung und Information (M 331)“ wurden aus dem Schwerpunkt 3 "Lebensqualität im ländlichen Raum und Diversifizierung der ländlichen Wirtschaft" folgende Ziele abgeleitet:

- Verbesserung der Lebensqualität in ländlichen Regionen

- Diversifikation wirtschaftlicher Aktivitäten

- Stärkung territorialer Kohärenz und Synergien

- Verbesserung des Humanpotentials zur Diversifikation der lokalen Wirtschaft und Bereitstellung von lokalen Serviceleistungen.

\subsubsection{Ziele Agrar-Umwelt und Naturschutz / Agrarumweltmaßnahmen (M 214)}

Im Rahmen der hier beschriebenen Bildungsevaluierung wurde jener Teil des Österreichischen Programms zur Förderung einer umweltgerechten, extensiven und den natürlichen Lebensraum schützenden Landwirtschaft (ÖPUL) - eingebettet in den Agrarumweltmaßnahmen M 214 - betrachtet, der sich auf Bildungsmaßnahmen der LE bezieht, die seit 2007 zu Themen aus diesem Programm angeboten wurden. Speziell wurden in der vorliegenden Studie in diesem Zusammenhang Bildungsangebote zum Thema "Biologische Wirtschaftsweise" hinsichtlich ihrer Wirkung auf die folgenden Ziele der Agrarumweltmaßnahmen betrachtet:

- Förderung der nachhaltigen Entwicklung des ländlichen Raums;

- Abdeckung der steigenden gesellschaftlichen Nachfrage nach Umweltdienstleistungen; 
- Ermutigung der LandwirtInnen, im Dienste der gesamten Gesellschaft Produktionsverfahren beizubehalten oder einzuführen, die mit dem Schutz und der Verbesserung der Umwelt, des Landschaftsbildes, des ländlichen Lebensraumes, der natürlichen Ressourcen, der Böden und der genetischen Vielfalt vereinbar sind.

\subsubsection{Ziele Forstbildungsmaßnahmen}

Die Zielsetzungen der Forstbildungsmaßnahmen setzen sich aus jenen der Maßnahmen 111 und 331 zusammen, betrachten dabei aber jeweils gesondert jene Bildungsangebote, die dem ForstBereich zuzuordnen sind (111c \& 111d und 331c \& 331d).

\subsection{Finanzierung}

Insgesamt wurden in den Maßnahmen M 111 und M 331 zwischen in den Jahren 2007-2012 $€ 75.484 .932,30$ an Fördergeldern ausbezahlt.

Im Förderungszeitraum 2007-2012 wurden in der Maßnahme 111 insgesamt Fördergelder in der Höhe von $€ 48.648 .841$,31 vergeben. Davon wurden 12,4\% als TeilnehmerInnen-Förderung (111a TeilnehmerInnen-Förderung Landwirtschaft \& 111c TeilnehmerInnen-Förderung Forstwirtschaft) vergeben, die restlichen $87,6 \%$ als Veranstalterförderung (111b Veranstalterförderung Landwirtschaft \& 111d Veranstalterförderung Forstwirtschaft) - siehe Abbildung 1. Abbildung 2 zeigt, welche Organisationen wie viel an Veranstalterförderung erhielten, gereiht nach Förderhöhe. Etwaige Kooperationen der angeführten Veranstalter mit anderen Institutionen wurden mangels Information dabei nicht erfasst.

Abbildung 1: Maßnahme 111 (Berufsbildung und Information) - Gesamtförderung

Gesamtförderung M111 (€48.619.561,31)

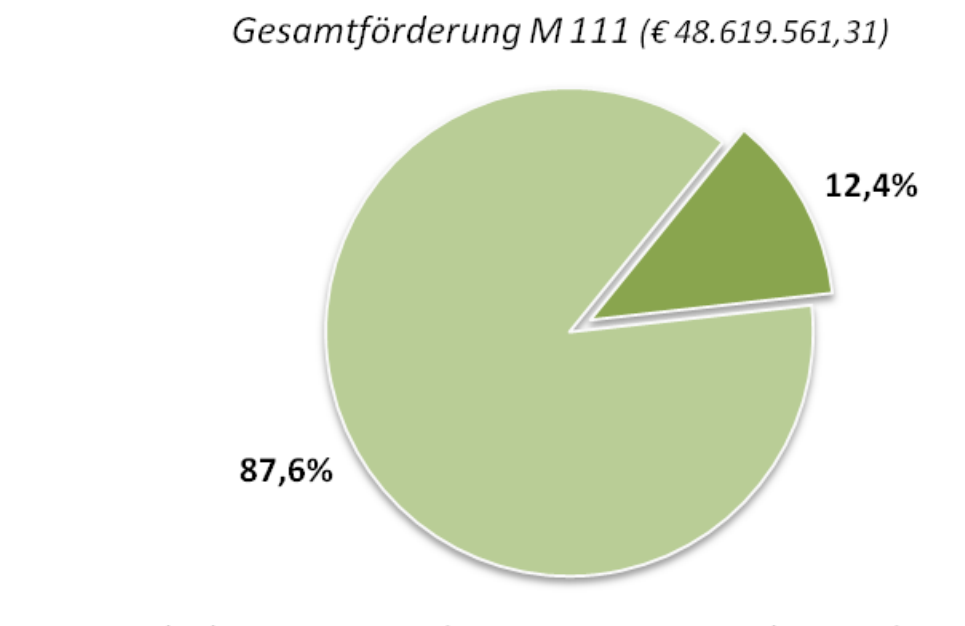

- TeilnehmerInnen-Förderung घVeranstalter-Förderung

Quelle (Daten): Bundesanstalt für Agrarwirtschaft (2013) / INVECOS 
Abbildung 2: Maßnahme 111 (Berufsbildung und Information) - Veranstalterförderung

Maßnahme 111 - Veranstalterförderung gesamt (M 111b + M 111d) 2007-2012 nach Veranstalter

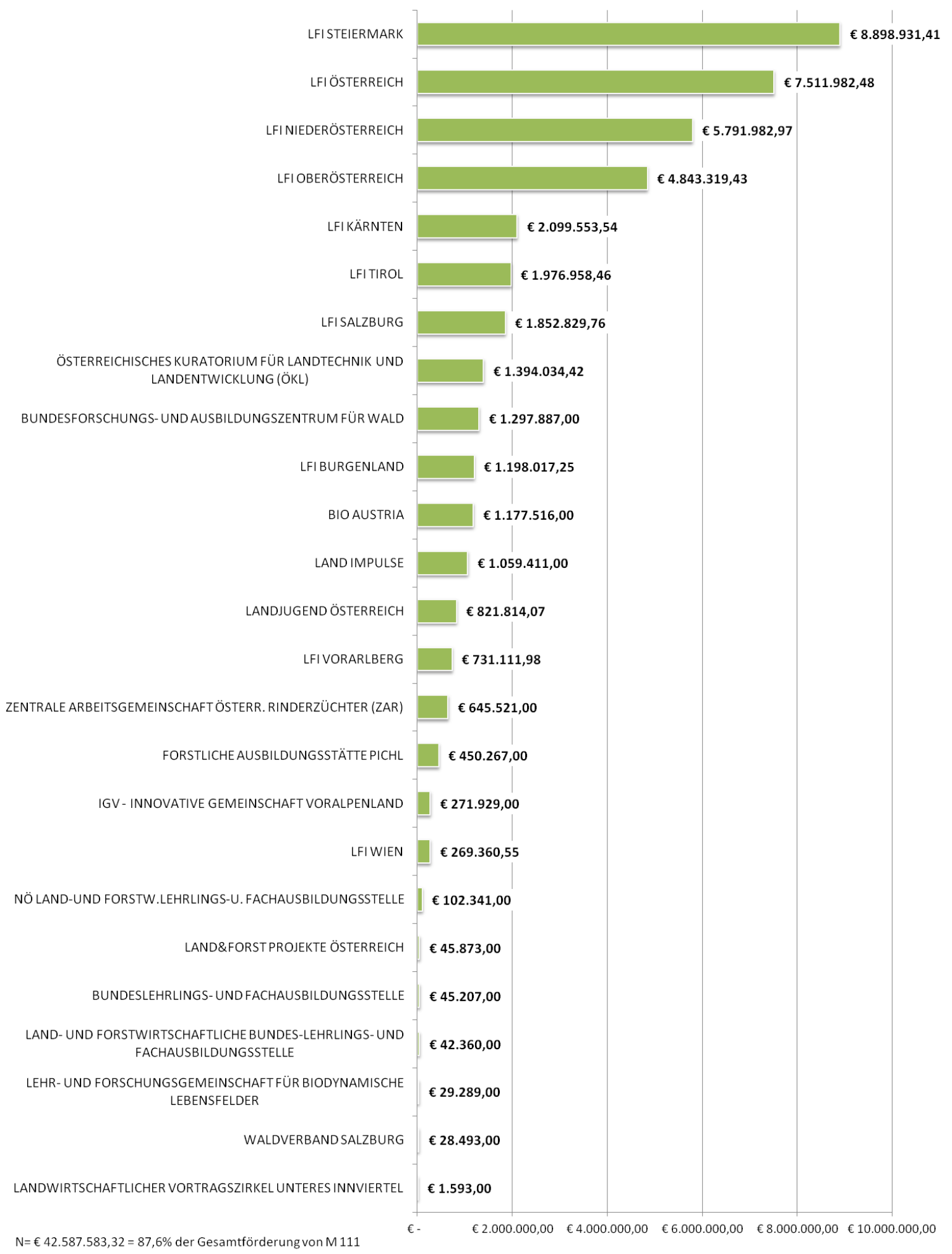

Quelle (Daten): Bundesanstalt für Agrarwirtschaft (2013) / INVECOS 
In Maßnahme 331 wurden in der laufenden Förderperiode LE 07-13 von 2007-2012 insgesamt $€$ 26.865.374,00 an Förderungen vergeben. Der prozentmäßige Anteil der TeilnehmerInnenFörderung (331a TeilnehmerInnen-Förderung Landwirtschaft \& 331c TeilnehmerInnen-Förderung Forstwirtschaft) beträgt dabei 4,6\%, die restlichen $95,4 \%$ wurden als Veranstalterförderung (331b Veranstalterförderung Landwirtschaft \& 331d Veranstalterförderung Forstwirtschaft) vergeben (Abbildung 3). Die Förderempfänger seitens der Veranstalter sind, gereiht nach Höhe der erhaltenen Förderung, in Abbildung 4 angeführt. Etwaige Kooperationen der angeführten Veranstalter mit anderen Institutionen wurden (mangels Information) nicht erfasst.

Abbildung 3: Maßnahme 331 (Ausbildung und Information) - Gesamtförderung

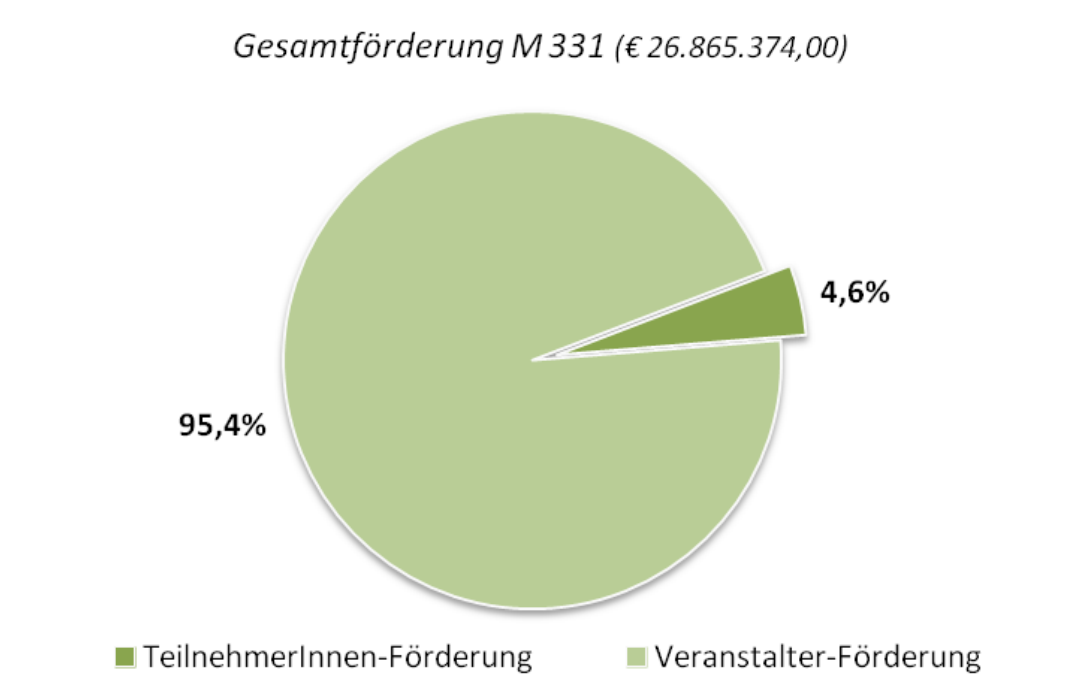

Quelle (Daten): Bundesanstalt für Agrarwirtschaft (2013) / INVECOS 
Abbildung 4: Maßnahme 331 (Ausbildung und Information) - Veranstalterförderung

Maßnahme 331 - Veranstalterförderung gesamt ( M 331b + M 331d) 2007-2012 nach Veranstalter

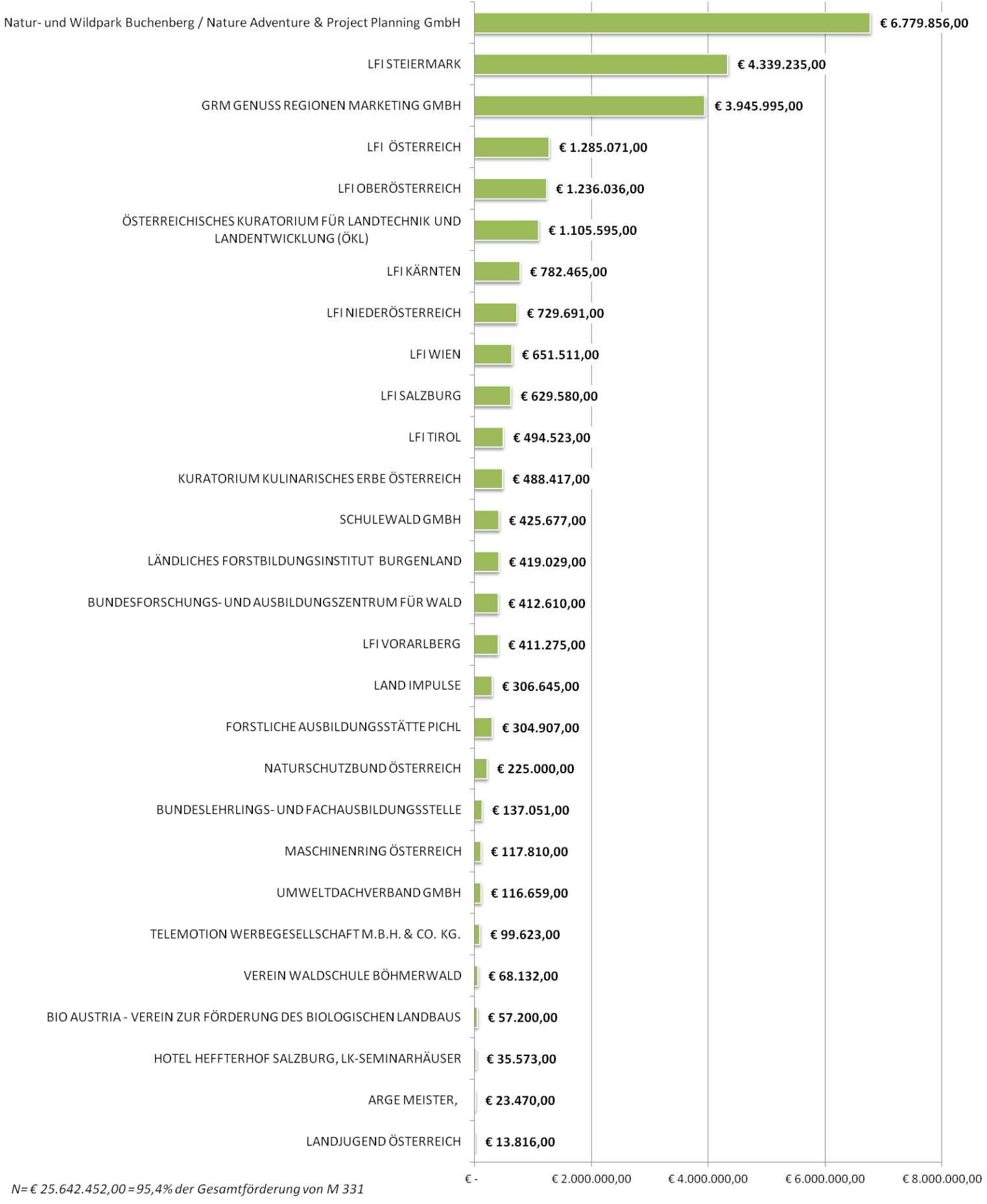

Quelle (Daten): Bundesanstalt für Agrarwirtschaft (2013) / INVECOS

Insgesamt erhielten somit nur $13,5 \%$ aller geförderten Veranstalter $60,7 \%$ der gesamten Veranstalterförderung. Nimmt man die $10 \mathrm{LFIs}$ als eine, zusammengehörige Organisation, dann erhielten überhaupt nur 3,6 \% aller geförderten Veranstalter $67 \%$ der gesamten Veranstalterförderung - eine bemerkenswerte Konzentration der Fördermittel. 


\section{VORGEHENSWEISE BEI DER EVALUIERUNG}

\subsection{Evaluierungsdesign}

Es wurden vier Methoden der empirischen Sozialforschung eingesetzt. Diese wurden so gewählt und eingesetzt, dass die Ergebnisse einer Methode mit Hilfe einer weiteren vertieft werden können und sich somit eine Schärfung ergibt. Die ausgewählten Methoden sind darauf abgestimmt, einander zu ergänzen, und als Methodenmix die Validität und Relevanz der Ergebnisse sicherzustellen.

Folgende Methoden kamen bei der Evaluierung zum Einsatz:

- Dokumentenanalyse

- Online-Umfrage unter Nutzung von http://de.surveymonkey.com/

- Dialog-Interviews - siehe etwa http://www.presencing.com/tools/dialogue-interviews

- Evaluation Cafés - siehe etwa http://www.weitzenegger.de/content/?page id=1781

\subsection{Durchführung}

Die Erhebungen zur Evaluierung begannen nach Auftragserteilung im Juli 2012. Die Unterlagen zur Dokumentenanalyse wurden einerseits vom Lebensministerium geliefert, andererseits stammen sie von der Webseite des Lebensministeriums. Dokumentenanalyse, Dialog-Interviews und Erstellung der Online-Umfrage fanden parallel zueinander statt. Der Online-Fragebogen wurde im Survey-Tool „SurveyMonkey" erstellt und war nach einem Testlauf von Anfang März bis Ende April 2013 geöffnet. Die drei Evaluation Cafés in Salzburg, Bruck a. d. Mur und Wien fanden im April und Mai statt. Die Auswertungen und Analysen der Evaluation Cafés und der Online-Umfrage wurden im Mai und Juni durchgeführt und in diesen Endbericht eingearbeitet. Das Projekt wurde seitens des Lebensministerium durch mehrere Steuerungsgruppen-Besprechungen begleitet.

\subsubsection{Dokumentenanalyse}

Diverse Unterlagen wurden in elektronischer Form durch das Lebensministerium übermittelt bzw. stammen von der Website des Ministeriums. Daneben wurden weitere Dokumente durch OnlineRecherchen ausfindig gemacht. Zusätzlich wurden von den Anbietern von Bildungsmaßnahmen (meist LFI) diverse Broschüren und Programmhefte zur Verfügung gestellt.

Eine Übersicht über alle analysierten Dokumente (mit Ausnahme der diversen Broschüren zum aktuellen Bildungsprogramm der verschiedenen Veranstalter) befindet sich in Anhang III.

\subsubsection{Online-Umfrage}

Der Fragebogen wurde zu 52,7\% (= 1.298 absolut) von Personen aus der Land- bzw. Forstwirtschaft beantwortet. Öffentliche Stellen und Veranstalter von Bildungsmaßnahmen sind zu 26,4\% vertreten. Weitere 20,9\% sind Personen aus anderen Branchen, die zum Teil auch Bildungsangebote in Anspruch genommen haben (siehe Abbildung 6). In diesem Kapitel wird graphisch ein Überblick über die Personen, die an der Online-Umfrage teilgenommen haben gegeben. Die Auswertungen zu den Fragestellungen des Lebensministeriums befinden sich in Kapitel 4. 
Abbildung 5: Teilnehmerlnnen am Fragebogen (gesamt)

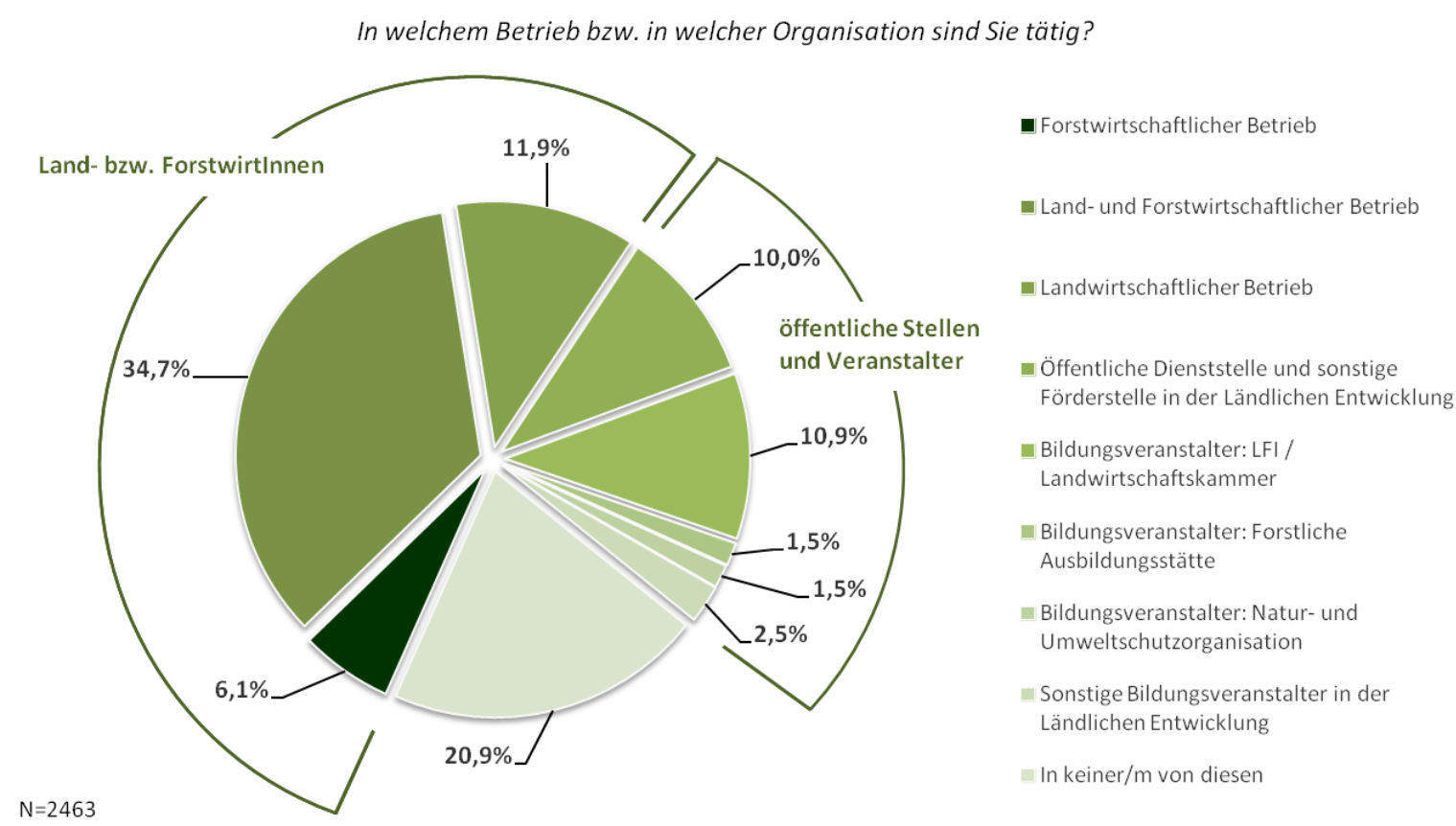

Der Fragebogen war auch jenen Personen zugänglich, die angaben, weder in der Land- und Forstwirtschaft noch in öffentlichen Dienststellen oder bei Veranstaltern tätig zu sein ( $N=515)$. Davon gaben 395 Personen (76,7\%) an, seit 2007 an Bildungsmaßnahmen der Entwicklung des Ländlichen Raumes teilgenommen zu haben. Diese Personen wurden ebenfalls zu ihren Erfahrungen und Einschätzungen bzgl. der Wirkung der Bildungsmaßnahmen befragt. Jenen übrigen 120 Personen, die an keinen Bildungsmaßnahmen teilgenommen haben, wurden keine weiteren Fragen gestellt, da für sie keine Berührungspunkte mit den Bildungsmaßnahmen bestehen.

Abbildung 6: Teilnehmerlnnen aus anderen Branchen - Besuch von Bildungsangeboten der LE

Haben Sie seit dem Jahr 2007 an Bildungsangeboten in der Ländlichen Entwicklung teilgenommen?

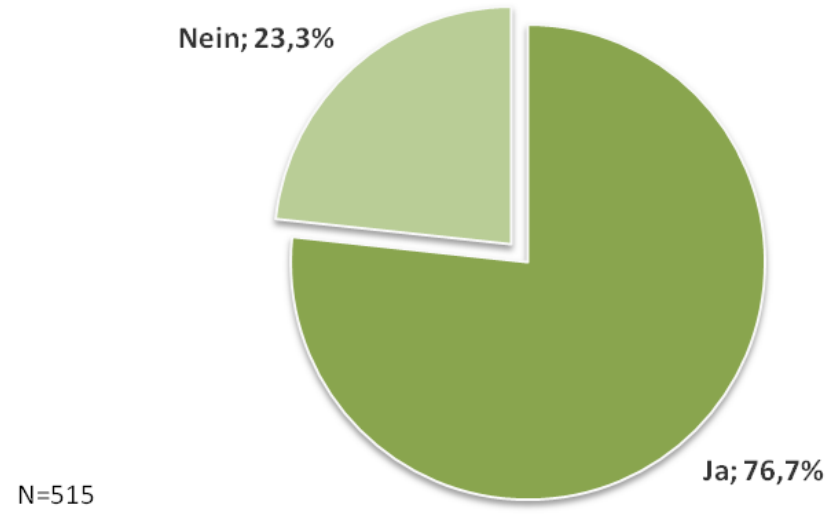

Die Beteiligung der Frauen am Fragebogen war geringer, als die der Männer. In Kapitel 4 wird auf die Unterschiede zwischen den weiblichen und männlichen TeilnehmerInnen am Fragebogen de- 
tailliert eingegangen. Dazu werden zu den einzelnen Fragestellungen jeweils vergleichende Graphiken präsentiert.

Abbildung 7: Aufteilung der TeilnehmerInnen (gesamt) nach Gender

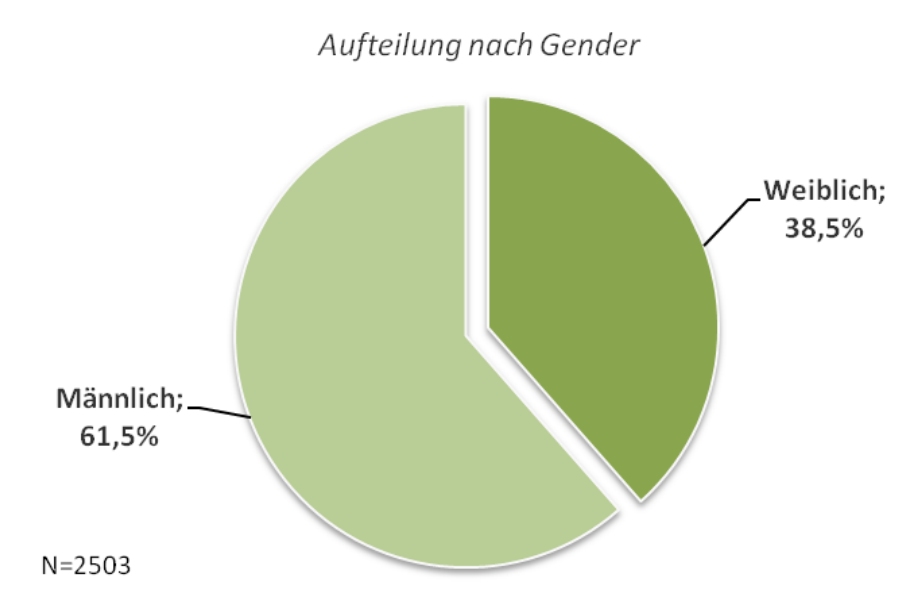

Die Altersverteilung zeigt, dass die größte Beteiligung in der Gruppe der 25-54jährigen liegt.

Abbildung 8: TeilnehmerInnen (gesamt) nach Altersgruppe

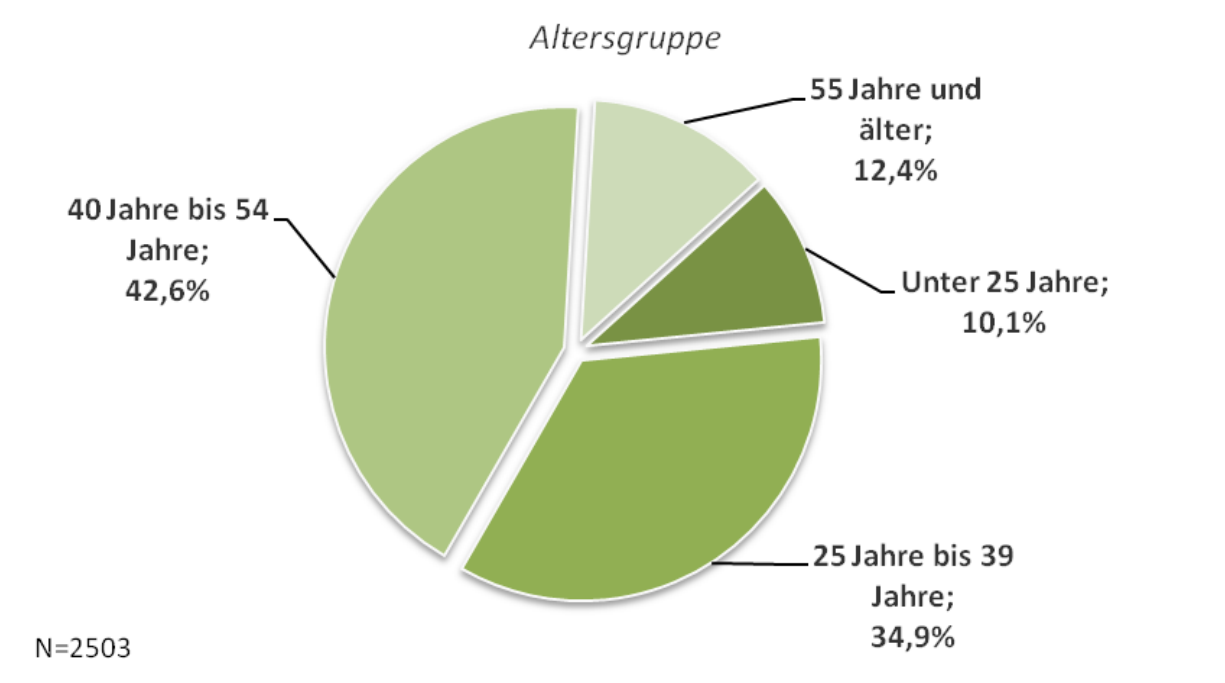

Die Bundesländer Oberösterreich und Steiermark sind am stärksten im Fragebogen vertreten. Wie Abbildung 9 zeigt, konzentrierte sich die Beteiligung an der Online-Umfrage vor allem auf den östlichen Teil Österreichs. 
Abbildung 9: Teilnehmerlnnen (gesamt) nach Bundesland

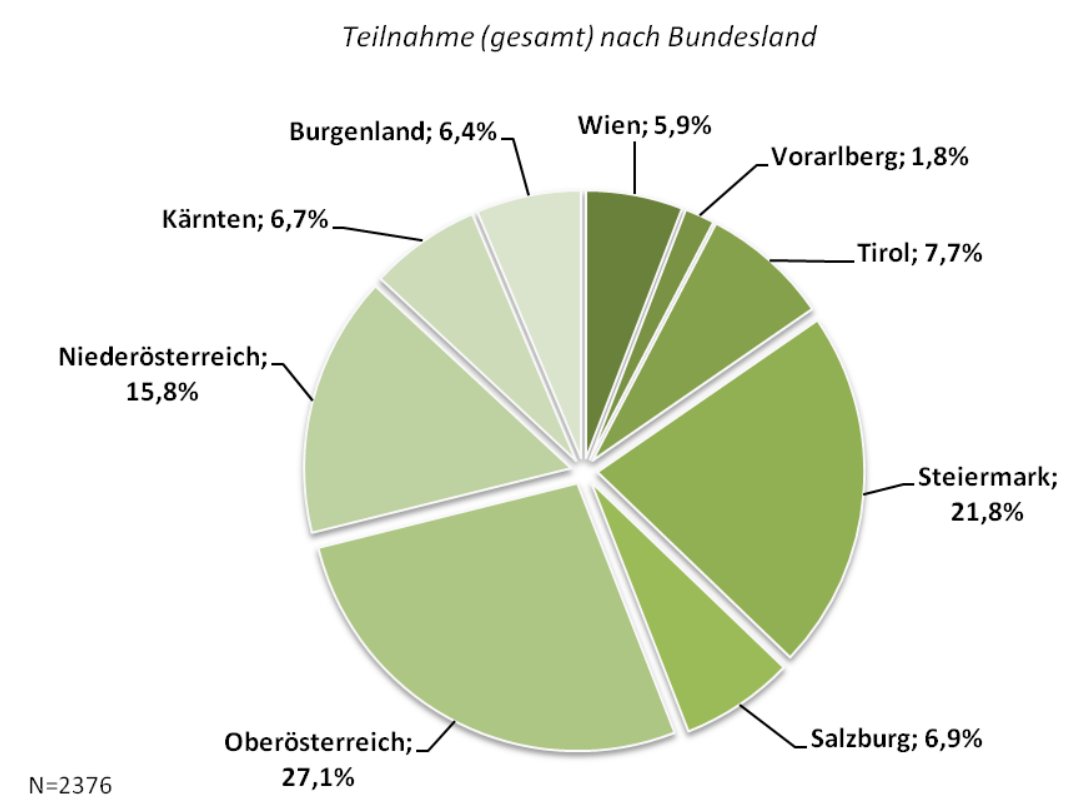

Die Verteilung der Land- bzw. ForstwirtInnen nach Bundesländern ist in nachstehender Abbildung 10 zu sehen.

Abbildung 10: TeilnehmerInnen (Land-/ ForstwirtInnen) nach Bundesland Teilnahme (Land-/ ForstwirtInnen) am Fragebogen nach Bundesland

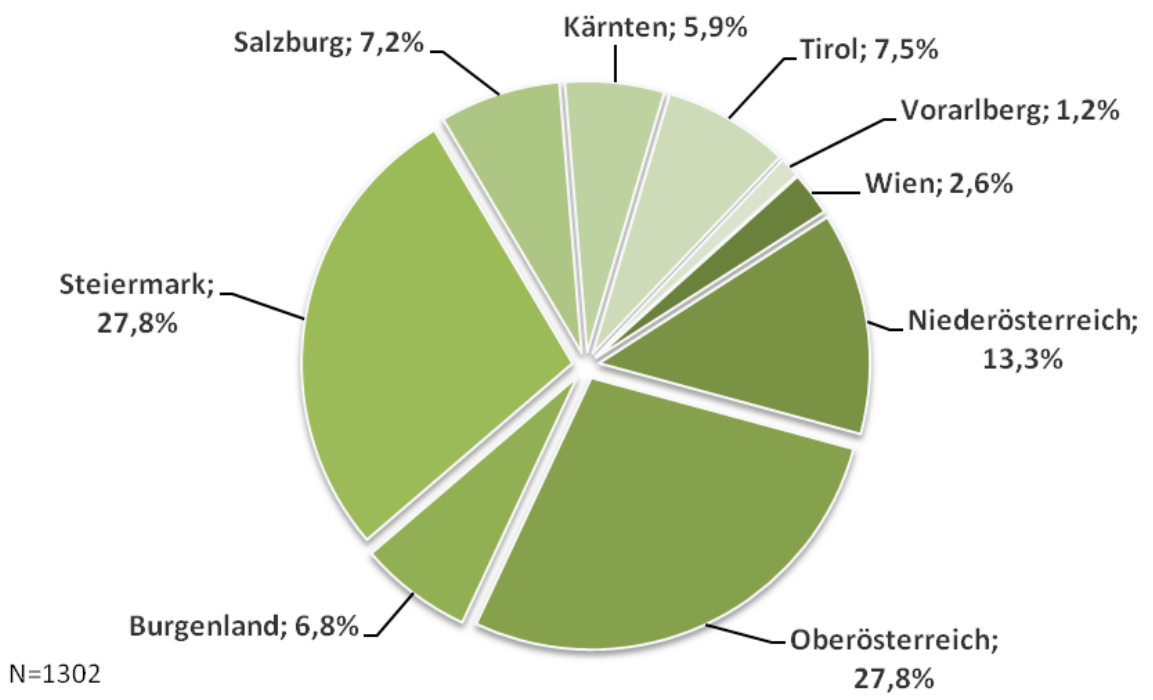

Ein Drittel der Personen, die nicht in der Land- oder Forstwirtschaft tätig sind, aber ebenfalls an Kursen der Ländlichen Entwicklung teilgenommen, und auch den Fragebogen beantwortet haben, kommt aus Oberösterreich (Abbildung 11). Auf diese Gruppe der Nicht-Land- bzw. NichtForstwirtInnen wird in Kapitel 4.11.3 näher eingegangen. 
Abbildung 11: TeilnehmerInnen (KursbesucherInnen aus anderen Branchen) nach Bundesland Teilnahme (KursbesucherInnen aus anderen Branchen) am Fragebogen nach Bundesland

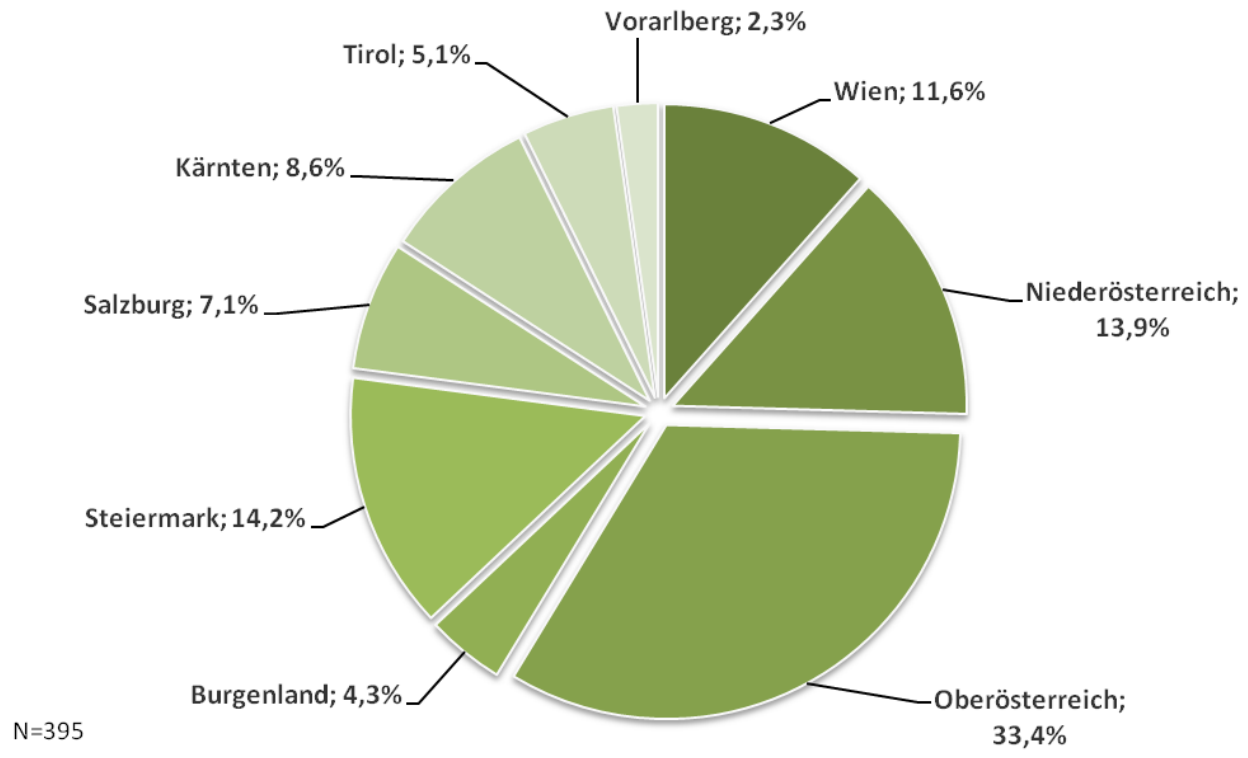

Die Bundesländer Oberösterreich, Steiermark und Niederösterreich sind auch in der Gruppe der Personen von öffentlichen Dienststellen und Bildungsveranstaltern am stärksten vertreten.

Abbildung 12: TeilnehmerInnen (öffentliche Dienststellen und Veranstalter) nach Bundesland

Teilnahme (öffentliche Dienststellen und Veranstalter) am Fragebogen nach Bundesland

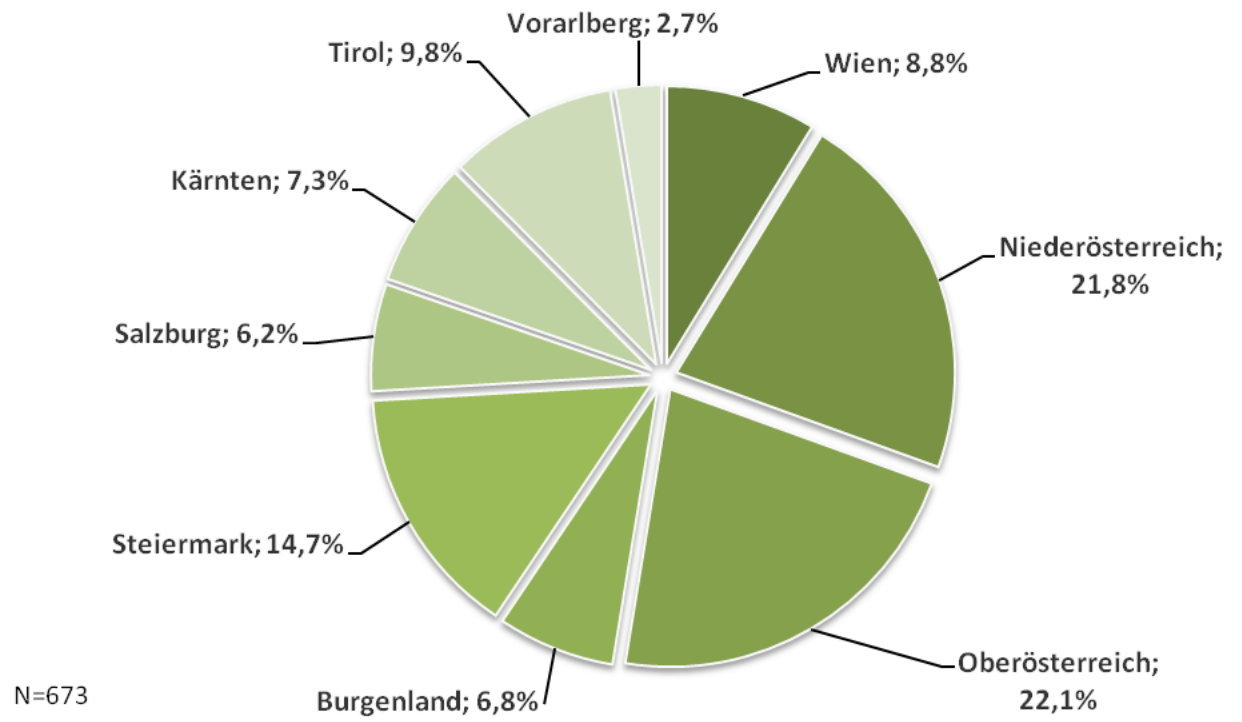


In Abbildung 13 ist die Verteilung aller Teilnehmer am Fragebogen nach Schulbildung zu sehen.

Abbildung 13: Aufteilung der Teilnehmerlnnen (gesamt) nach Schulbildung

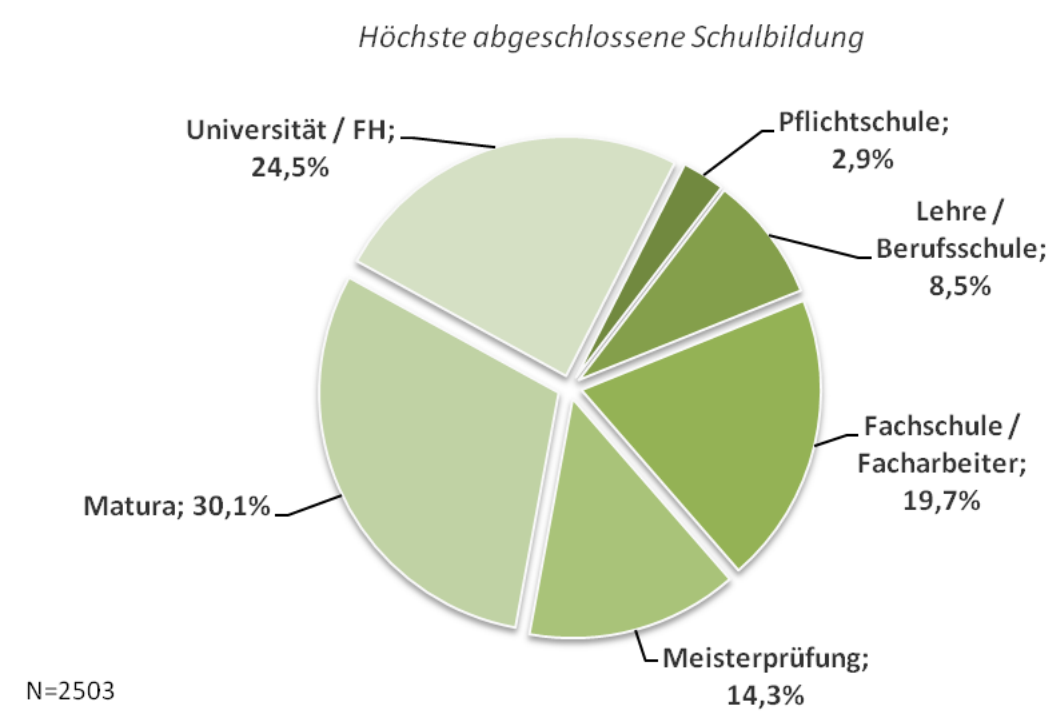

\subsubsection{Dialog-Interview}

Beim Dialog-Interview handelt es sich um einen neuen Typus von Interview - siehe etwa http://www.presencing.com/tools/Dialog-interviews -, das zum Ziel hat, nicht nur bestehende Informationen und Meinungen von der interviewten Person in Erfahrung zu bringen, sondern während und durch das Interview zu neuen Einsichten und Erkenntnissen zu gelangen. Diese Form ist dem sogenannten Tiefeninterview nicht unähnlich, hat aber einen strikt fachlichen und keinen persönlichen Fokus. Vorteil des Dialog-Interviews ist, dass ehrliche, offene Antworten gegeben werden und nicht nur solche, von denen die interviewte Person glaubt, dass sie vom Interviewer bzw. vom dahinter stehenden Auftraggeber erwünscht sind. In der Regel dauert ein solches Interview 1,5- 2 Stunden.

Besonders auf Fragestellungen zu Verbesserungspotentialen bzw. -vorschlägen und zur Wirkung des Programms lassen sich durch diese Methode hochwertige und weiterführende Antworten gewinnen.

Im Zuge der Evaluierung wurden 40 Dialog-Interviews durchgeführt. Diese Interviews wurden Großteils als Einzelinterviews, teilweise auch als Paarinterviews, mit Personen, die vom Auftraggeber als Interviewpartner vorgeschlagen wurden, durchgeführt. Eine Übersicht über die Interviewpartner, sowie Details zu den Interviewterminen finden sich im Technischen Anhang in Beilage I.

\subsubsection{Evaluation Café}

Dies ist eine besonders partizipative Form der Fokusgruppe, welche sich vom World Café ableitet. Sie wird genutzt, um einer Gruppe von Personen (bei einer Gruppengröße von ca. 25 Personen) zu ermöglichen, sich über spezifische Fragen der Evaluation auszutauschen. Der Nutzen dieser Methode besteht darin, dass in nur 2 Stunden die Sichtweisen von ca. 25 Personen, die in unterschiedlichem Bezug zum Programm Ländliche Entwicklung stehen, in Erfahrung gebracht werden können. Der Nutzen für die Teilnehmenden besteht im intensiven Erfahrungsaustausch. 
Im Rahmen der in diesem Bericht beschriebenen Evaluierung der Bildungsmaßnahmen der LE wurden drei Evaluation Cafés, jeweils eines in Salzburg, Bruck a. d. Mur und in Wien organisiert. Die Aussendung der Einladungen zu den Evaluation Cafés erfolgte im März und April 2013 durch das Lebensministerium. Eine Übersicht über die TeilnehmerInnen an den Evaluation Cafés findet sich in Beilage II des technischen Anhangs.

Bei jedem Evaluation Café konnten die TeilnehmerInnen in mehreren Runden zu folgenden drei Fragestellungen ihre Erfahrungen und Ideen austauschen. Die Fragen wurden gemeinsam mit der Steuerungsgruppe des BMLFUW erarbeitet.

(1) Was hat im geförderten Bildungsbereich im Programm Ländliche Entwicklung viel bewirkt? (2) Es wurden zwei alternative Fragestellungen formuliert, die bei den Cafés alternierend gestellt wurden:

a. Auf welche großen, ungelösten Herausforderungen der Entwicklung des ländlichen Raums sollten sich die zukünftigen Fördermaßnahmen von Wissenstransfer und Innovation konzentrieren?

b. Auf welche großen, ungelösten Herausforderungen bei Wissenstransfer und Innovation zur Entwicklung des ländlichen Raums sollten sich die zukünftigen Fördermaßnahmen konzentrieren?

(3) Wie sollte das Lebensministerium im Rahmen von LE 2020 Wissenstransfer und Innovation gestalten, um möglichst viel zu bewirken?

Die Notizen der TeilnehmerInnen zu den einzelnen Fragen sowie die zusammenfassenden Aussagen der Kleingruppen wurden in einem Fotoprotokoll dokumentiert und jeweils an die Teilnehmerlnnen versandt. Die Ergebnisse aus den drei Evaluation Cafés flossen zudem in die Kapitel 4 und 5 dieses Evaluationsberichtes mit ein. Die erwähnten Fotoprotokolle finden sich ebenfalls im Technischen Anhang, Beilage III. 


\section{ERGEBNISSE}

Für die Beantwortung der Fragestellungen hinsichtlich der Wirkungen der Bildungsmaßnahmen wird zunächst besonders auf die Antworten von Land- bzw. ForstwirtInnen aus dem Fragebogen und den Interviews eingegangen. Um ein klareres Bild dieser Land- bzw. ForstwirtInnen zu erhalten, werden zunächst einige Hintergrundinformationen zu diesen angeführt.

Die Graphiken beziehen sich im Folgenden jeweils auf Auswertungen der Online-Befragung.

Im Anschluss an die Auswertungen der Antworten der Land- bzw. ForstwirtInnen werden im Kapitel 4.11 Weitere Analyseresultate die Antworten seitens der TeilnehmerInnen aus anderen Branchen sowie der Bildungsveranstalter und öffentlichen Dienststellen präsentiert.

Die gendermäßige Verteilung der Land- bzw. ForstwirtInnen spiegelt die zuvor in Abbildung 7 gezeigte Verteilung der Gesamt-Teilnehmer am Fragebogen wider.

Abbildung 14: Aufteilung der Land- bzw. ForstwirtInnen nach Gender

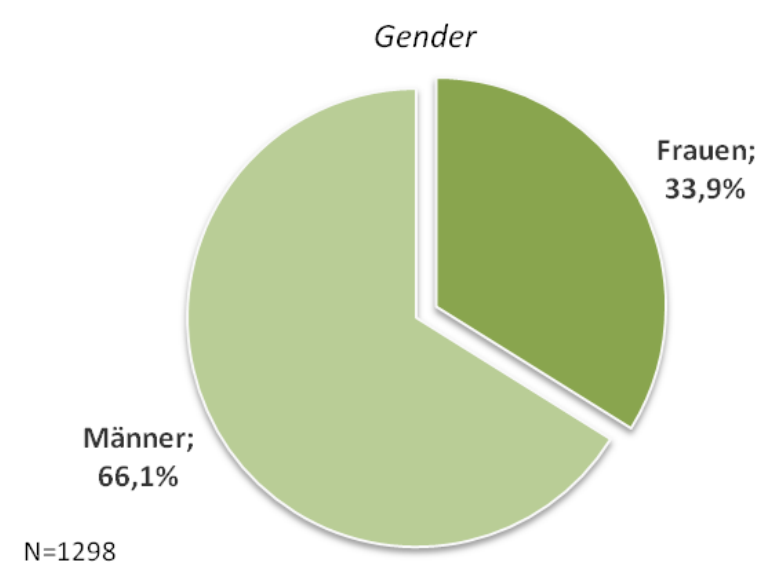

Auch die Verteilung nach Altersgruppen zeigt große Ähnlichkeiten mit der Verteilung in Abbildung 8.

Abbildung 15: Aufteilung der Land-bzw. ForstwirtInnen nach Altersgruppe

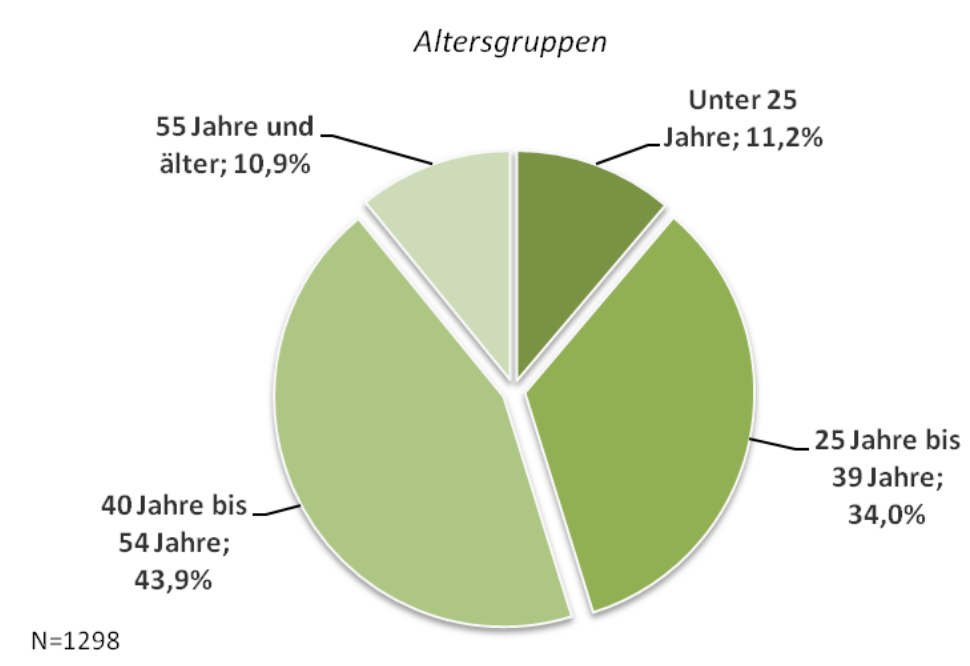


Die meisten der teilnehmenden Land- bzw. ForstwirtInnen gaben bei der Frage nach der höchsten abgeschlossene Schulbildung an, die Matura gemacht zu haben. Fachschule / Facharbeiter und Meisterprüfung kommen dabei an zweiter und dritter Stelle.

Abbildung 16: Aufteilung der Land- bzw. ForstwirtInnen nach Schulbildung

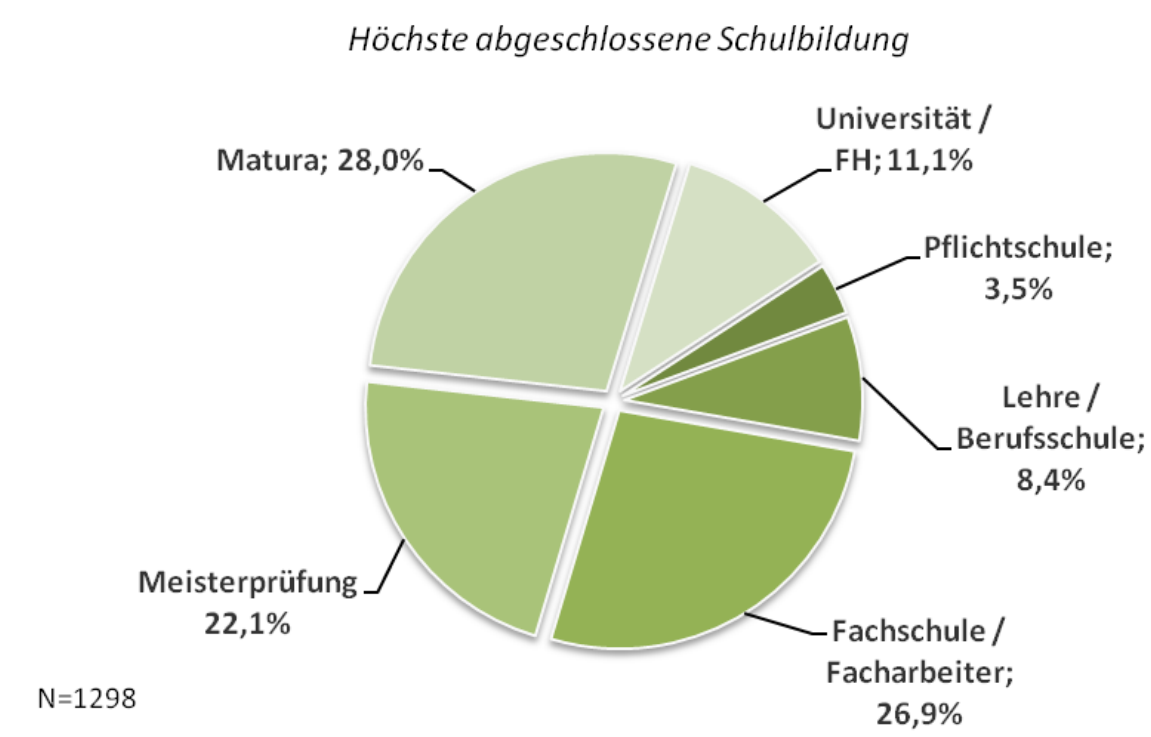

Von den teilnehmenden Land- bzw. ForstwirtInnen sind mehr als die Hälfte im Haupterwerb als solche tätig.

Abbildung 17: Aufteilung der Land-bzw. ForstwirtInnen nach Haupt- und Nebenerwerb In welchem Ausmaß sind Sie in diesem Betrieb tätig?

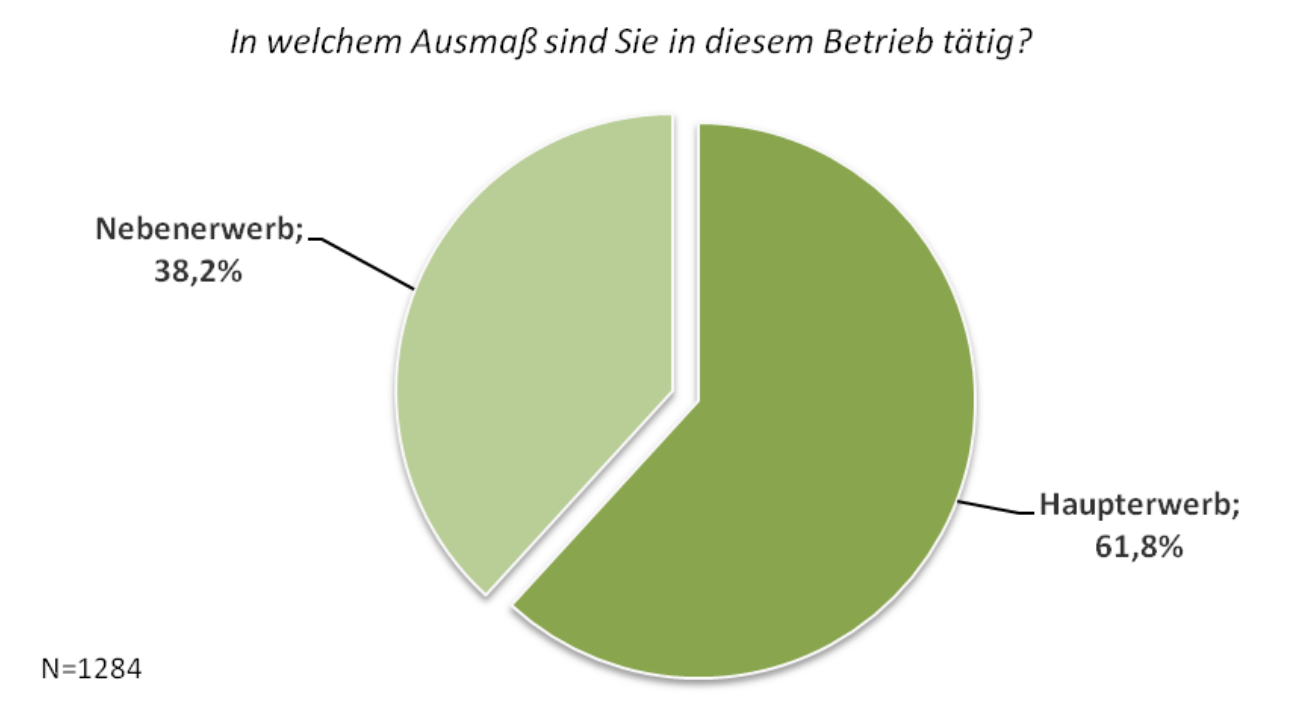


Mehr als ein Viertel der teilnehmenden Land- bzw. ForstwirtInnen sind in einem Bio-Betrieb tätig.

Abbildung 18: Anteil Bio-Betriebe

Ist dieser Betrieb ein Bio-Betrieb?

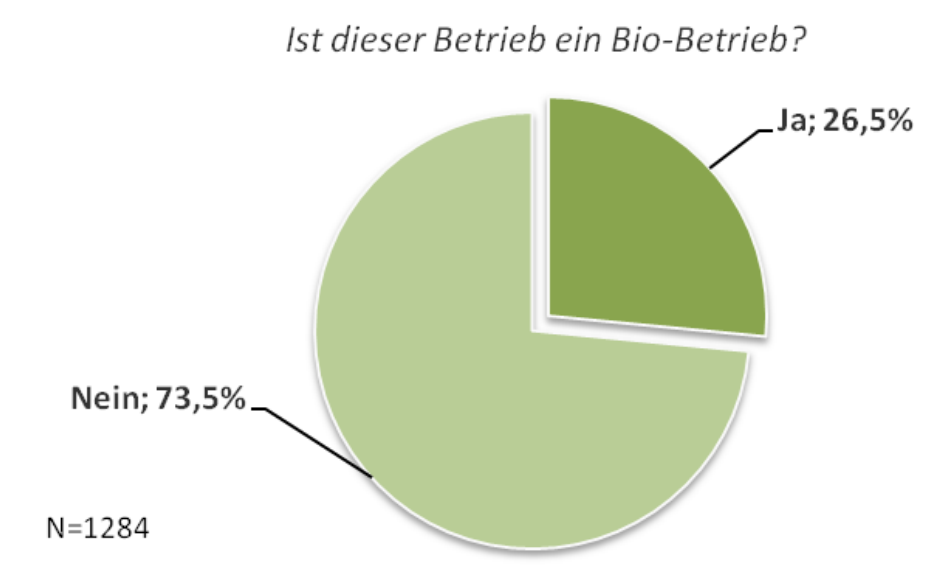

Daneben wurden die Land- bzw. ForstwirtInnen gebeten anzugeben, ob sie zusätzlich in nichtagrarischen Bereichen tätig sind (Abbildung 19). Diese Frage wurde von etwas mehr als der Hälfte $(50,8 \%)$ der Befragten verneint. Es konnten dabei maximal zwei Antwortmöglichkeiten gewählt werden. Die meisten Nennungen derjenigen, die auch in anderen Bereichen tätig sind, erhielt die Kategorie "Tourismus, Gastronomie und Direktvermarktung".

\section{Abbildung 19: Zusatzbereiche}

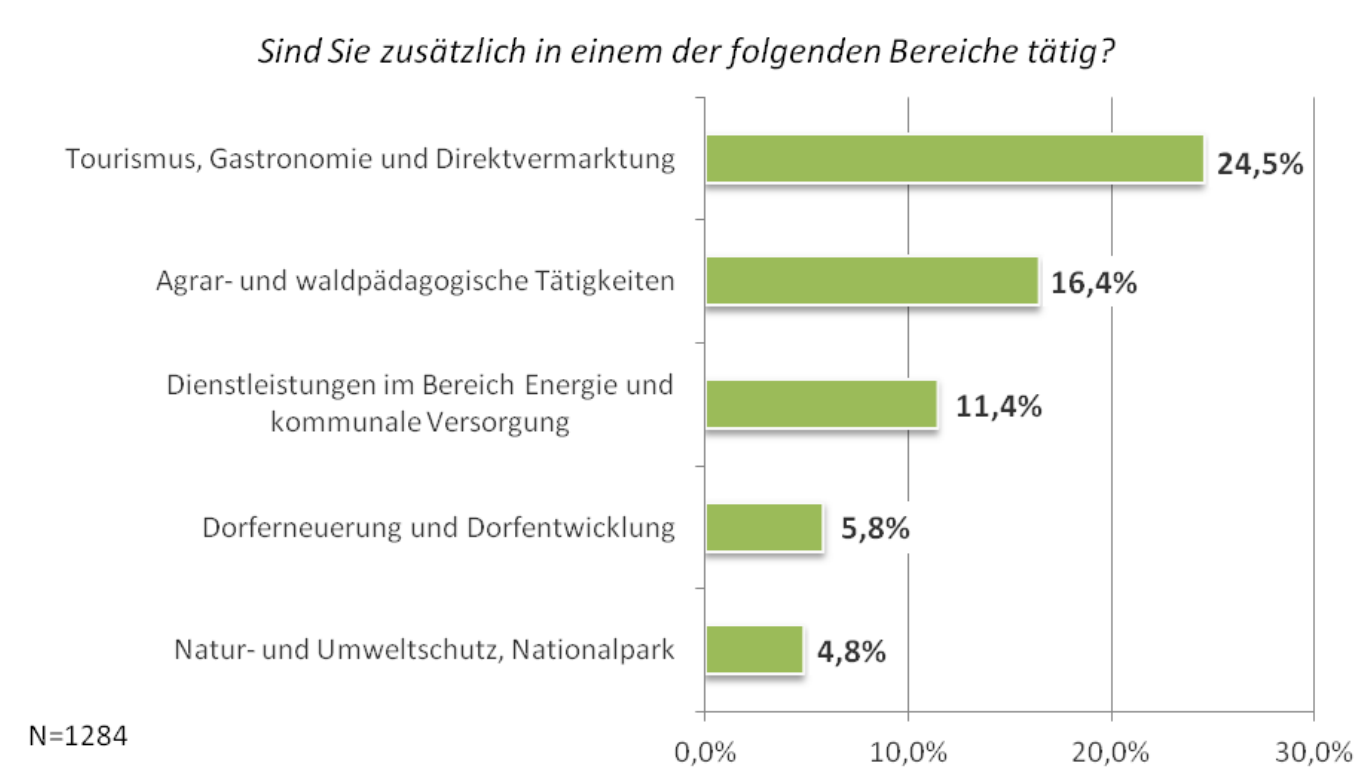

Die überwiegende Mehrheit der Land- bzw. ForstwirtInnen nahm in der Programmperiode 07-13 an Bildungsveranstaltungen der Ländlichen Entwicklung teil (siehe Abbildung 20). 
Abbildung 20: Teilnahme an Bildungsveranstaltungen der LE 07-13

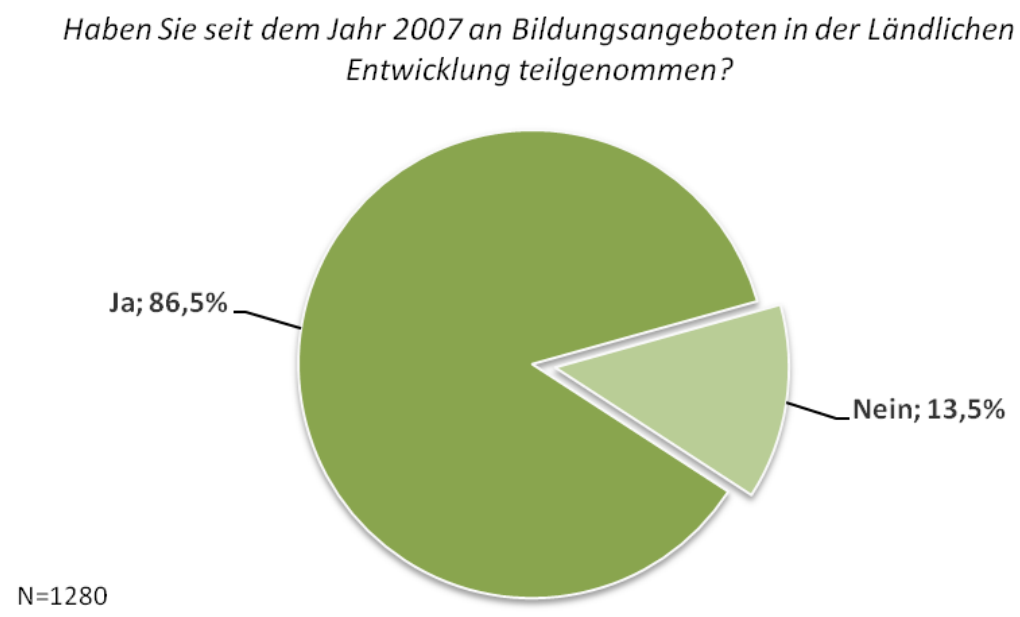

Dazu wurde in den Dialog Interviews folgendes erwähnt:

„Die Tendenz, Weiterbildung zu machen, ist steigend."

Jene Land- bzw. ForstwirtInnen, die angaben, keine Bildungsangebote in Anspruch genommen zu haben, wurden gebeten, Gründe für diese Entscheidung zu nennen. Dabei konnten mehrere Antwortmöglichkeiten gewählt werden. Das nach der Kategorie "Aus einem anderen Grund" meist genannte Hindernis zur Inanspruchnahme von Bildungsangeboten ist die mangelnde Zeit. Rund $20 \%$ derjenigen, die diese Frage gestellt bekamen gaben an, keine Informationen zu Bildungsangeboten erhalten zu haben. Weitere $19 \%$ können für sich keinen speziellen Nutzen, den eine Teilnahme an Bildungsangeboten der ländlichen Entwicklung bringen könnte, erkennen.

Dieser Gruppe der Land- bzw. ForstwirtInnen wurden in weiterer Folge ebenfalls Fragen zur Einschätzung des zu verschiedenen Themen zur Verfügung stehenden Bildungsangebotes, sowie zur Veränderung der persönlichen / beruflichen Situation seit 2007 gestellt.

Abbildung 21: Begründung für die Nicht-Teilnahme an Bildungsangeboten der LE

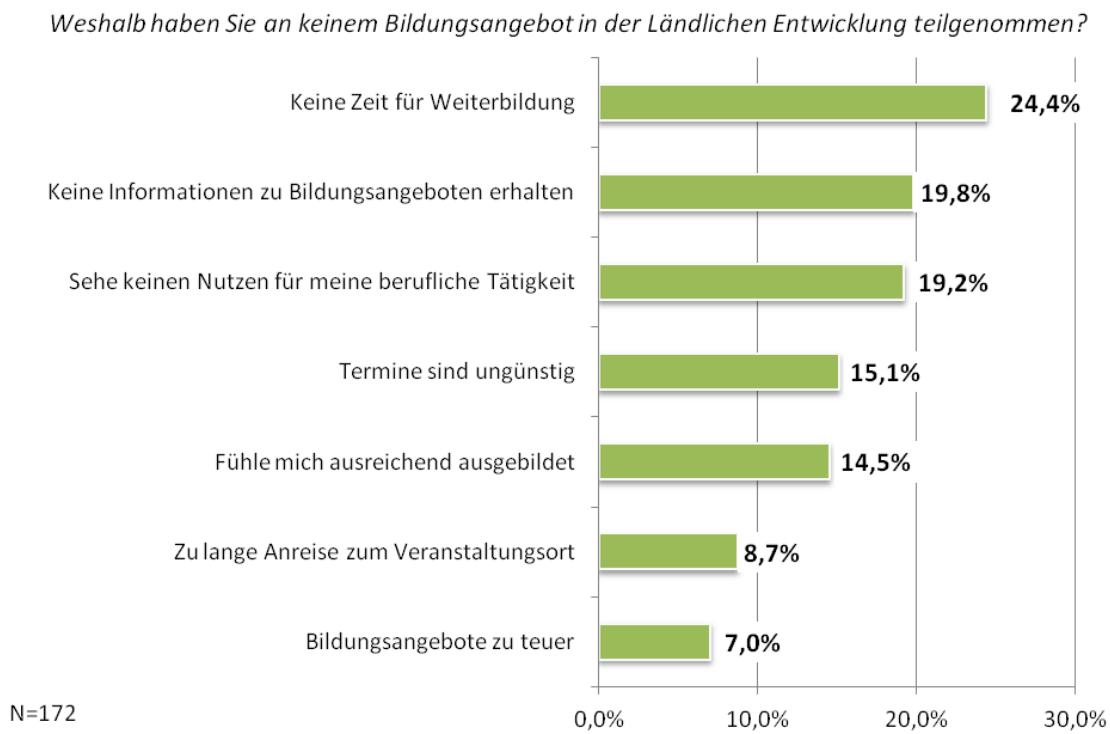


Bei den Begründungen lassen sich Unterschiede in den Antworten der Personen mit unterschiedlichen schulischen Abschlüssen feststellen. Dabei muss jedoch auch auf die kleinen GruppengröBen ( $\mathrm{N}$; siehe Legende in Abbildung 22) hingewiesen werden.

\section{Abbildung 22: Begründung für die Nicht-Teilnahme an Bildungsangeboten nach höchster abgeschlossener Schulbildung}

Weshalb haben Sie an keinem Bildungsangebot in der Ländlichen Entwicklung teilgenommen?

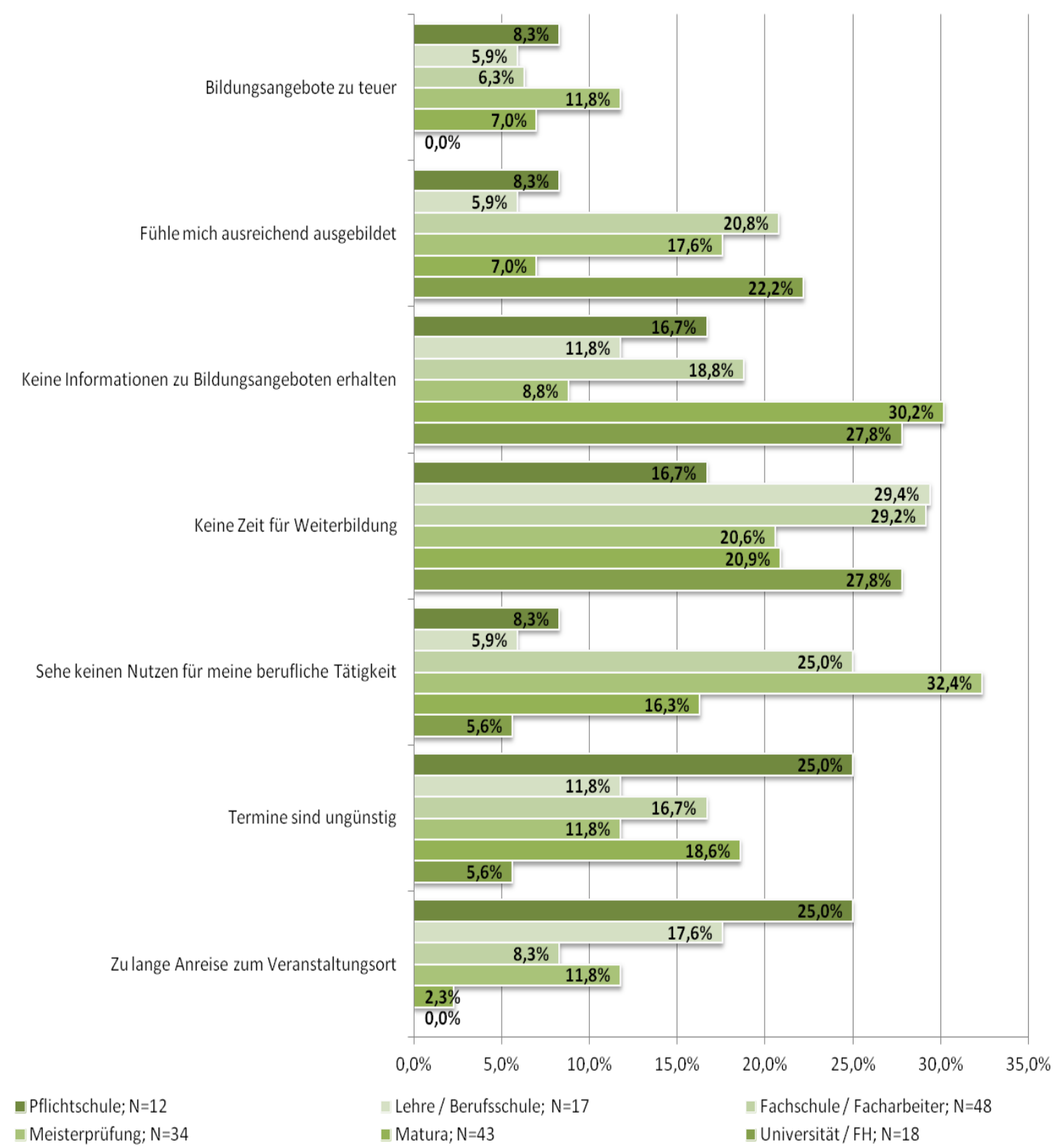

Abbildung 23 zeigt, bei welchen Veranstaltern die Bildungsangebote in Anspruch genommen wurden. Dabei konnten die Befragten mehrere Veranstalter auswählen. Die Meisten (77,6\%) der befragten Land- bzw. ForstwirtInnen haben Kurse der LFIs besucht. 
Abbildung 23: Veranstalter, bei welchen die Land-bzw. ForstwirtInnen Kurse der LE besucht haben

Bei welchem Veranstalter haben Sie Bildungsangebote der LE besucht?

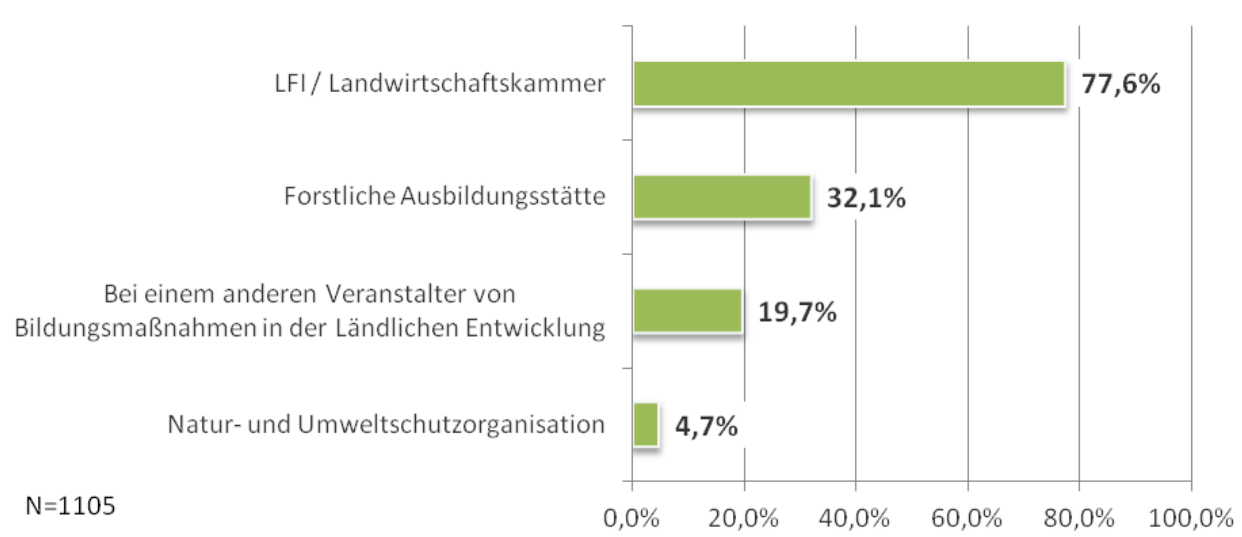

Bzgl. der Bildungsveranstalter wurde in den Dialog Interviews folgendes gesagt:

„Außer in NÖ werden in allen Ländern nur vom LFI Kurse angeboten. LFI monopolisiert Kurse in den Bundesländern."

Entsprechend des Auftrages zielt diese Studie auf die Wirkungen der Bildungsmaßnahmen differenziert nach den vier Bildungsmaßnahmen-Kategorien Agrar-Umwelt und Naturschutz, Forstbildungsmaßnahmen, Agrarische Bildungsmaßnahmen (M 111) sowie Bildungsmaßnahme M 331 ab. Eine solche Differenzierung ist methodisch diffizil und nur dadurch möglich, dass die Personen, welche den Fragebogen beantworteten, in Gruppen bzw. Kohorten aufgeteilt werden, entsprechend den von ihnen primär in Anspruch genommenen Bildungsmaßnahmen-Kategorien. Die folgende Einteilung erfolgt daher, um die Meinungen und Äußerungen der Personen zu den verschiedenen Bildungsangeboten möglichst klar trennen zu können. Kriterien der Gruppen- bzw. Kohortenbildung sind jene Bildungsangebote, die sich eindeutig den Bildungsmaßnahmen AgrarUmwelt und Naturschutz bzw. Forstbildungsmaßnahmen zuordnen lassen.

Zur Beantwortung der, ab Kapitel 4.2 behandelten Fragestellungen des Auftraggebers werden daher die im Folgenden präsentierten Ergebnisse des Fragebogens jeweils aus Sicht der folgenden drei Gruppen betrachtet:

(a) Personen, welche Bildungsmaßnahmen im Bereich Agrar-Umwelt und Naturschutz besucht haben, im Folgenden kurz Agrar-Umwelt und Naturschutz bzw. in den Abbildungen Agrar-Umwelt genannt;

(b) Personen, welche Forstbildungsmaßnahmen besucht haben, aber ohne jene Personen der Gruppe (a), im Folgenden kurz Forstbildungsmaßnahmen bzw. in den Abbildungen Forst genannt ;

(c) Personen, welche Bildungsmaßnahmen M 111 / M 331 besucht haben aber ohne die Personen der Gruppen (a) und (b), im Folgenden kurz Bildungsmaßnahmen M 111 \& M 331 bzw. in den Abbildungen M111 \& M331 genannt.

Die Aufteilung in die Gruppen basiert somit auf den gewählten Antwortmöglichkeiten zur Frage "Zu welchen der folgenden Themen haben Sie Bildungsangebote besucht?", die jeweils den Inhalten der oben genannten Maßnahmen entsprechen und dementsprechend zugeordnet werden können (siehe auch Abbildungen 48, 50 und 52 in Kapitel 4.4). Die Einteilung basiert somit auf den 
besuchten Kursen, und nicht auf der Herkunft (Land- oder forstwirtschaftlicher Betrieb) der TeilnehmerInnen, weil der Einfluss der Bildungsmaßnahmen auf die TeilnehmerInnen untersucht wird. Daneben erfolgt eine Gegenüberstellung der Antworten weiblicher und männlicher TeilnehmerInnen (Land- bzw. ForstwirtInnen) am Fragebogen, sowie - wo möglich und sinnvoll - eine Gegenüberstellung aller Land- bzw. ForstwirtInnen, die in der Periode 07-13 Bildungsangebote der LE in Anspruch genommen haben versus jenen Land- bzw. ForstwirtInnen, die keine Kurse besucht haben.

Kapitel 4.11.2 geht auf die Auswertungen der Fragen, die sich an die öffentlichen Dienststellen und Bildungsveranstalter richteten ein und stellt Vergleiche zwischen den Antworten der Landbzw. ForstwirtInnen und den Angaben und Einschätzungen der Veranstalter dar. Zusätzlich wird in Kapitel 4.11.3 die Analyse um Auswertungen der Antworten jener Personen ergänzt, die nicht in land- bzw. forstwirtschaftlichen Betrieben tätig sind, aber dennoch Bildungsangebote der LE in Anspruch genommen haben.

\subsection{Veränderungen für Land- bzw. ForstwirtInnen seit 2007}

Um mögliche Wirkungen der Bildungsmaßnahmen zu identifizieren, wurden die Teilnehmerlnnen gebeten, die seit 2007 bemerkten Veränderungen betreffend verschiedener Aspekte rund um den Betrieb bzw. den Ländlichen Raum mittels der Kategorien "weniger", "unverändert" und "mehr" einzuschätzen. Dieses Kapitel geht auf die Gesamt-Auswertung dieser Frage ein, die nachfolgenden Kapitel beschäftigen sich, neben weiteren Fragestellungen, mit Detailauswertungen zu einzelnen Antwortkategorien aus dieser Fragestellung.

Die nachstehende Abbildung 24 zeigt eine Gegenüberstellung der drei Gruppen (Agrar-Umwelt und Naturschutz, Forstbildungsmaßnahmen, M 111 \& M 331) bezüglich der Antworten zu dieser Frage. Die dargestellten Werte können dabei im Bereich zwischen $-100 \%$ und $+100 \%$ Wert liegen. Dabei bedeutet $-100 \%$, dass alle Personen "weniger" angeben, $+100 \%$, dass alle Personen "mehr" angeben. Ein Wert dazwischen ist der jeweilige Mittelwert, wobei die Angabe „unverändert" mit $0 \%$ quantifiziert wird.

Aus Abbildung 24 ist nun ersichtlich, dass der Mittelwert der Antworten in jeder Kategorie und für jede Personengruppe eine Zunahme gegenüber dem Jahr 2007 ergibt, wobei die geringste Zunahme in der Kategorie „Einkommenssituation" bei jenen Personen, welche Forstbildungsmaßnahmen besucht haben, zu verzeichnen ist und die höchste Zunahme in der Kategorie "Naturschutz- und umweltrelevantes Denken und Handeln" bei jenen Personen, welche Bildungsmaßnahmen im Bereich Agrar-Umwelt und Naturschutz besucht haben, zu verzeichnen ist. 
Abbildung 24: Veränderungen für Land- bzw. ForstwirtInnen seit 2007 - nach Bildungsmaßnahme

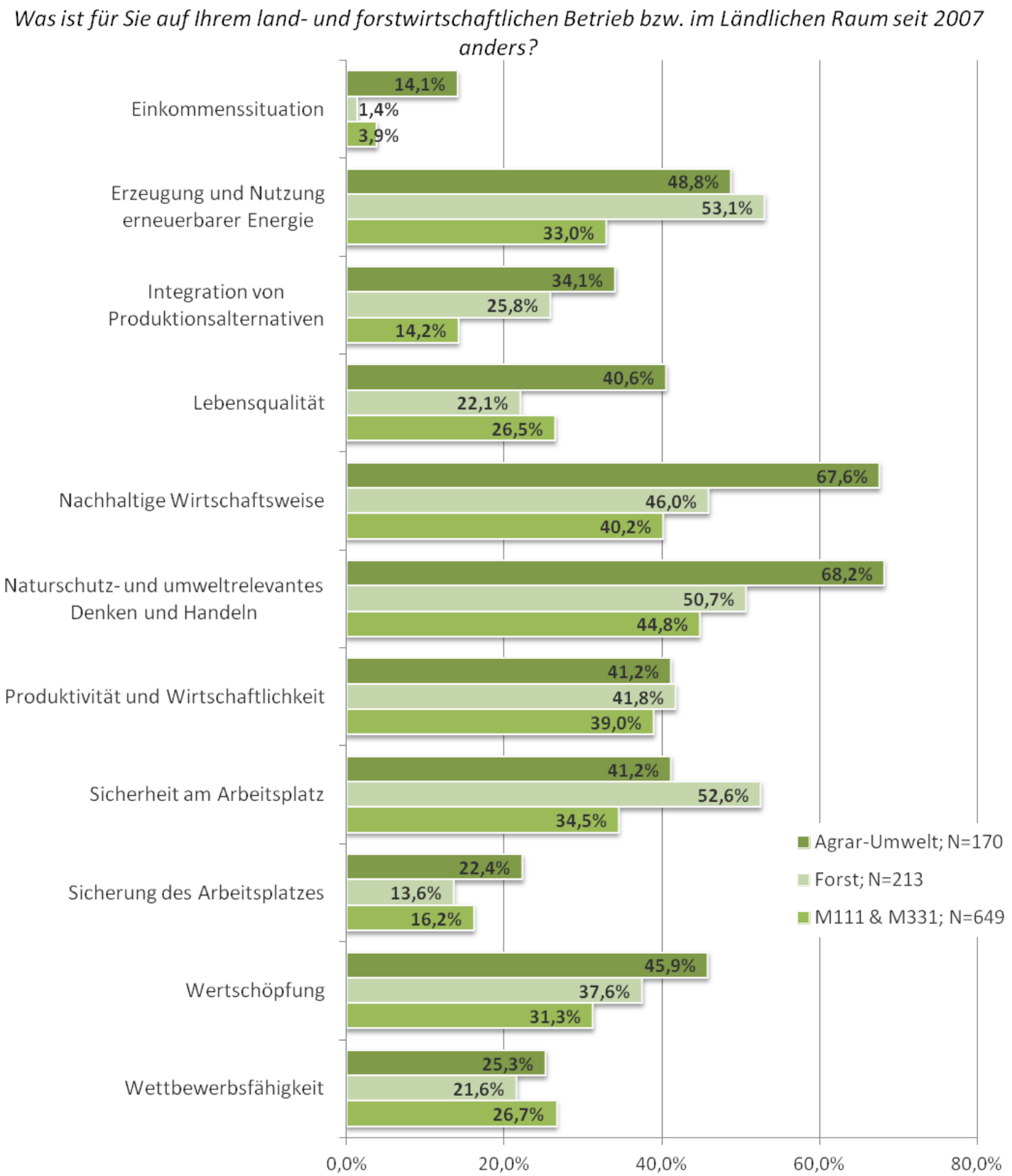

Die folgende Abbildung 25 zeigt die Antworten jener Personen, die seit 2007 an Bildungsangeboten teilgenommen haben im Vergleich zu jenen, die angaben, an keinen Bildungsangeboten teilgenommen zu haben. Dabei lassen sich merkbare Unterschiede feststellen. Jedoch ist auch festzuhalten, dass die Gruppe der Land- bzw. ForstwirtInnen, die keine Bildungsangebote besucht haben im Fragebogen weitaus geringer vertreten war ( $N=136)$, als die Vergleichsgruppe, die die Bildungsangebote der LE in Anspruch genommen hat ( $N=1030)$, wodurch sich eine gewisse Unschärfe ergibt.

Abbildung 25 ist somit ein zentrales Ergebnis dieser Studie, da ein wechselseitig verstärkender Zusammenhang zwischen dem Besuch von Bildungsmaßnahmen und der Einschätzung bezüglich der 11 zu Auswahl stehenden Kategorien empirisch feststellbar ist. Dies bedeutet, wie bei allen 
statistischen Korrelationen, nicht, dass eine Ursache-Wirkungs-Beziehung in die eine und/oder andere Richtung besteht, aber es bedeutet doch, dass alle jene Land- bzw. ForstwirtInnen, welche seit 2007 Bildungsmaßnahmen besucht haben, eine deutlich höhere Einschätzung bezüglich der positiven Veränderungen der 11 Kategorien haben als jene Land- bzw. ForstwirtInnen, die keine Bildungsangebote besucht haben, wobei der stärkste Zusammenhang in der Kategorie "Wertschöpfung" und der geringste Zusammenhang in der Kategorie „Erzeugung und Nutzung erneuerbarer Energie" festzustellen ist.

Abbildung 25: Veränderungen für Land- bzw. ForstwirtInnen seit 2007 - nach Teilnahme

Was ist für Sie auf Ihrem land- und forstwirtschaftlichen Betrieb bzw. im Ländlichen Raum seit 2007 anders?

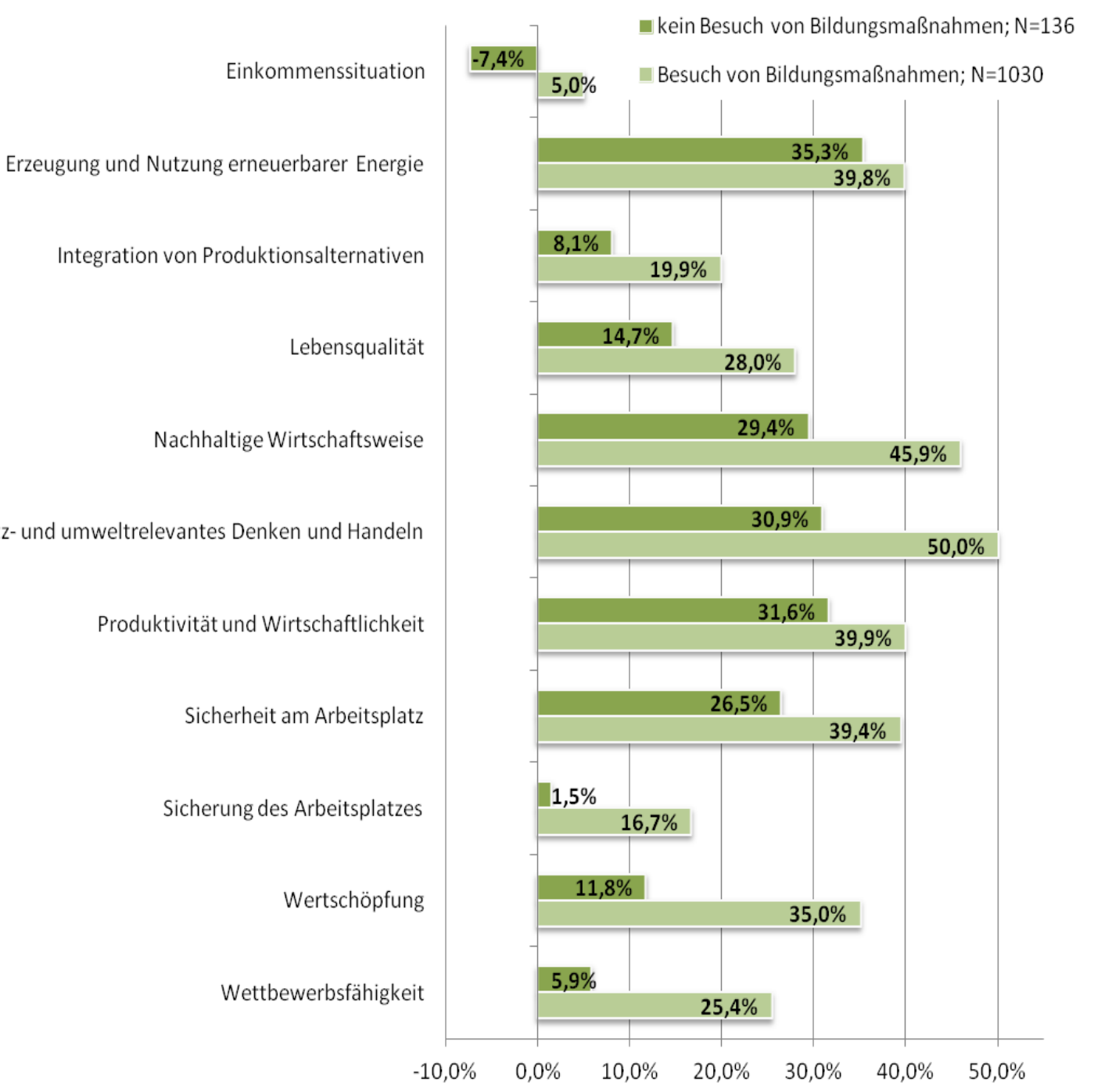


Vergleichsweise geringere Einschätzungs-Unterschiede zeigen sich zwischen Frauen und Männern, am deutlichsten jedoch bei der Erzeugung und Nutzung erneuerbarer Energie sowie bei der Lebensqualität.

Abbildung 26: Gegenüberstellung der Einschätzungen zwischen 2007 und 2013 nach Gender

Was ist für Sie auf Ihrem land- und forstwirtschaftlichen Betrieb bzw. im Ländlichen Raum seit 2007 anders?

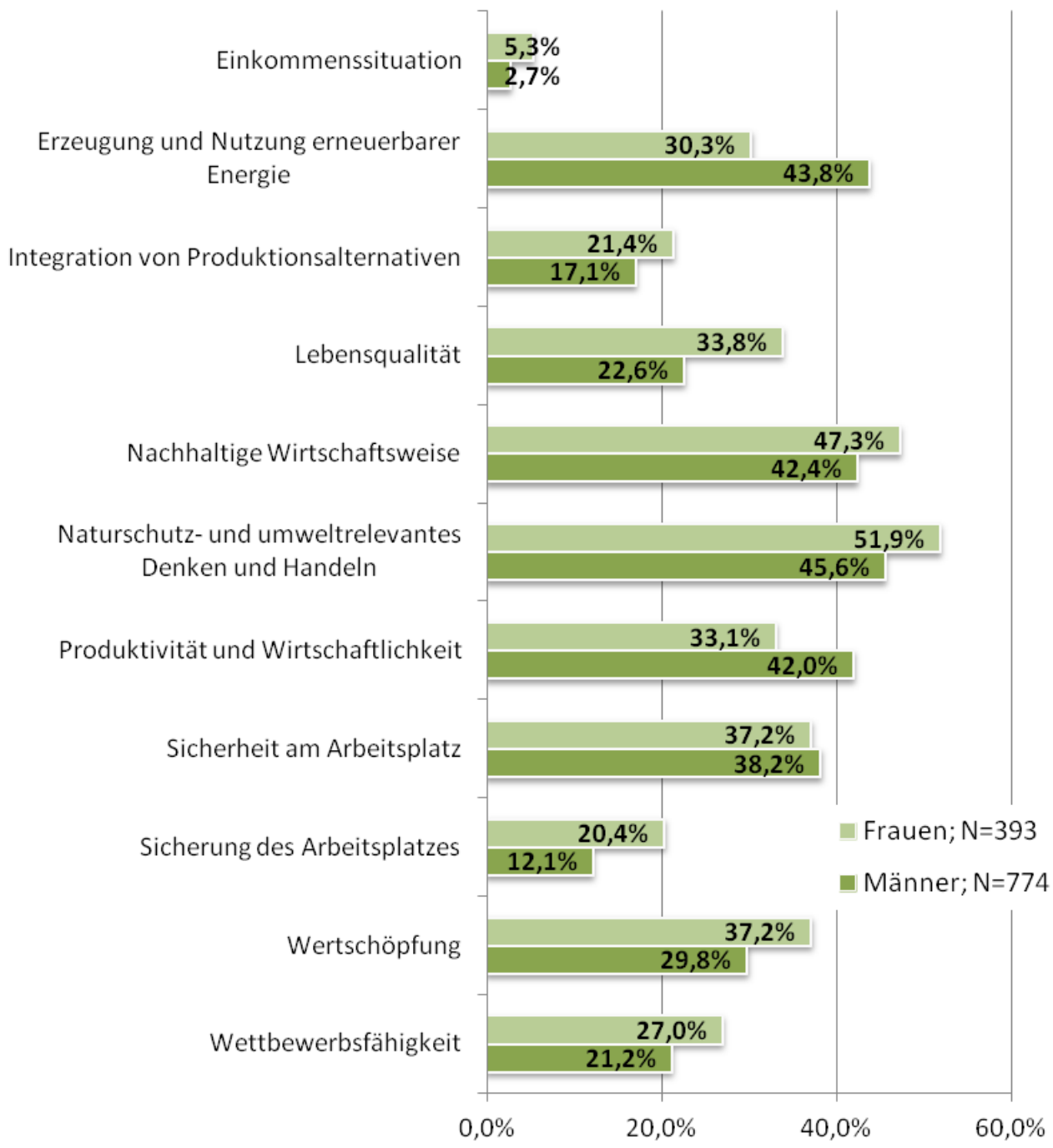

In den nachfolgenden Unterkapiteln werden nun die zuvor beschriebenen Gruppen im Detail sowie vergleichend hinsichtlich der konkreten Fragestellungen des Auftraggebers betrachtet. 


\subsection{Wettbewerbsfähigkeit in der Land-, Ernährungs- oder Forstwirtschaft}

\section{Bildungsmaßnahmen M 111 \& M 331}

Der Großteil der Land- bzw. ForstwirtInnen, die Kurse der Maßnahmen 111 und 331 besuchten, schätzt die Wettbewerbsfähigkeit als unverändert bzw. vermehrt ein.

Abbildung 27: Veränderungen bzgl. Wettbewerbsfähigkeit seit 2007 - M 111 \& M 331

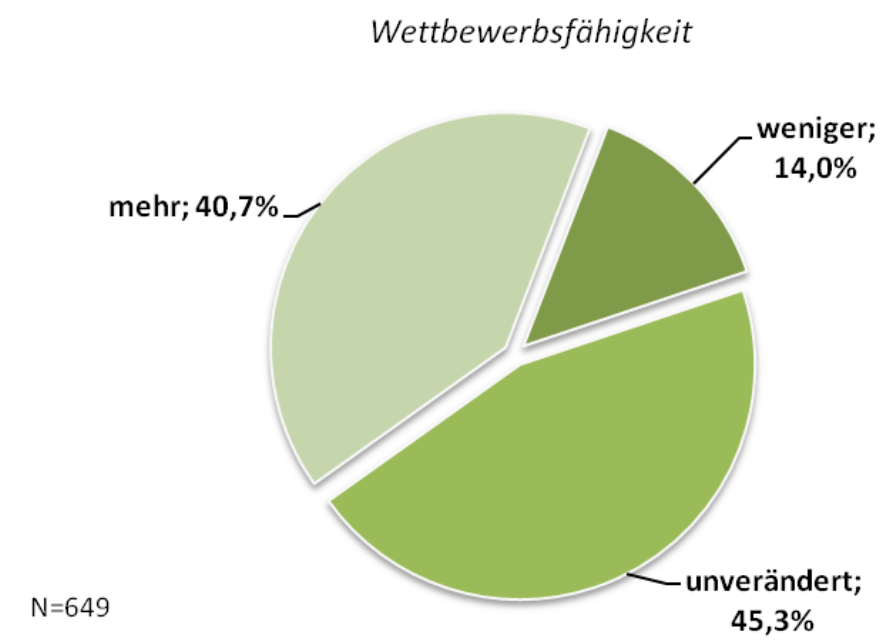

\section{Agrar-Umwelt und Naturschutz}

Bei jenen, die Bildungsangebote zu Agrar-Umwelt und Naturschutz in Anspruch genommen haben, sieht die Bewertung sehr ähnlich aus.

Abbildung 28: Veränderungen bzgl. Wettbewerbsfähigkeit seit 2007 - Agrar- Umwelt und Naturschutz

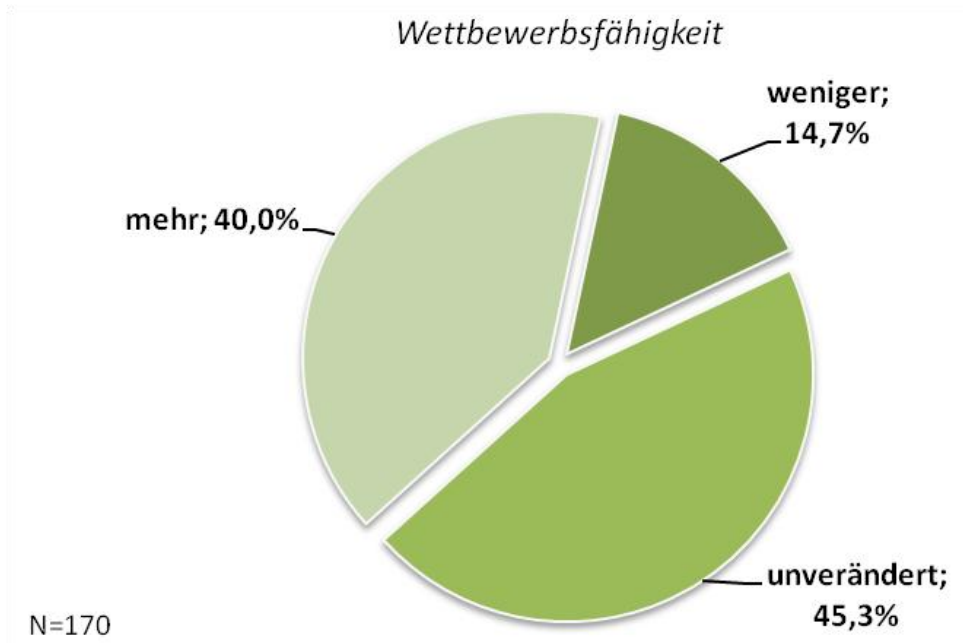




\section{Forstbildungsmaßnahmen}

Bei den Personen, die Bildungsangebote im Bereich Forst besucht haben, schätzt mehr als die Hälfte die eigene Wettbewerbsfähigkeit als unverändert im Vergleich zu der Zeit vor 2007 ein.

Abbildung 29: Veränderungen bzgl. Wettbewerbsfähigkeit seit 2007 - Forstbildungsmaßnahmen

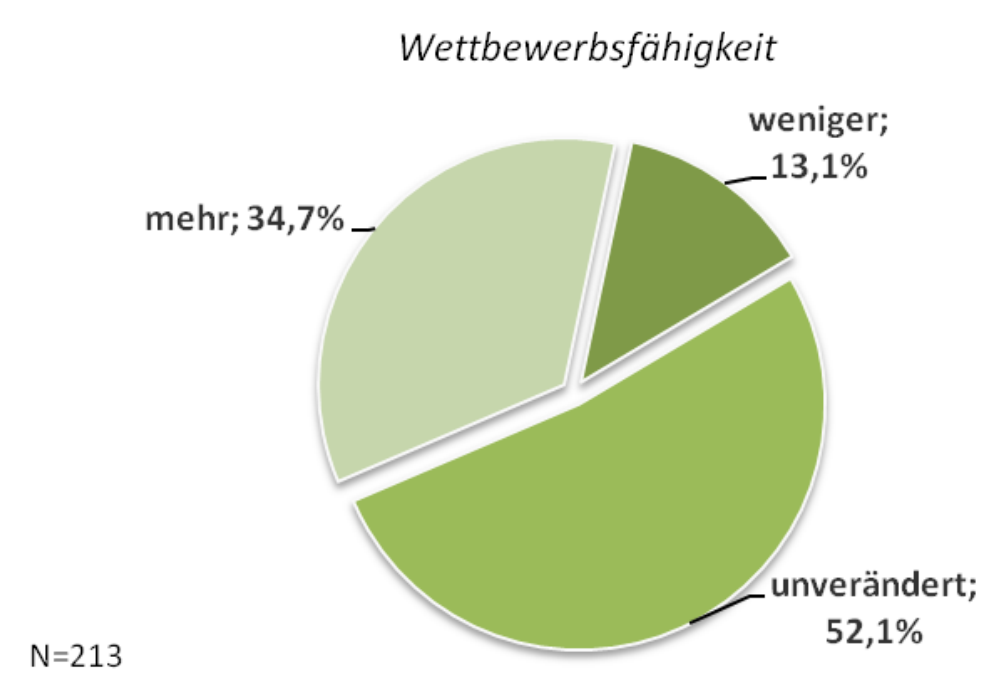

Land- bzw. ForstwirtInnen, die keine Bildungsangebote besucht haben

Bei der Gruppe von Personen, die in der Online-Befragung angaben, keine Kurse der LE besucht zu haben, wurde die Wettbewerbsfähigkeit, verglichen mit den vorhergehenden Darstellungen stärker mit „weniger" beurteilt. Hier ist auch der Anteil der Personen, welche die Bewertungskategorie „mehr" wählten geringer als bei den zuvor beschriebenen Gruppen.

Abbildung 30: Veränderungen bzgl. Wettbewerbsfähigkeit seit 2007 - keine Bildungsangebote besucht

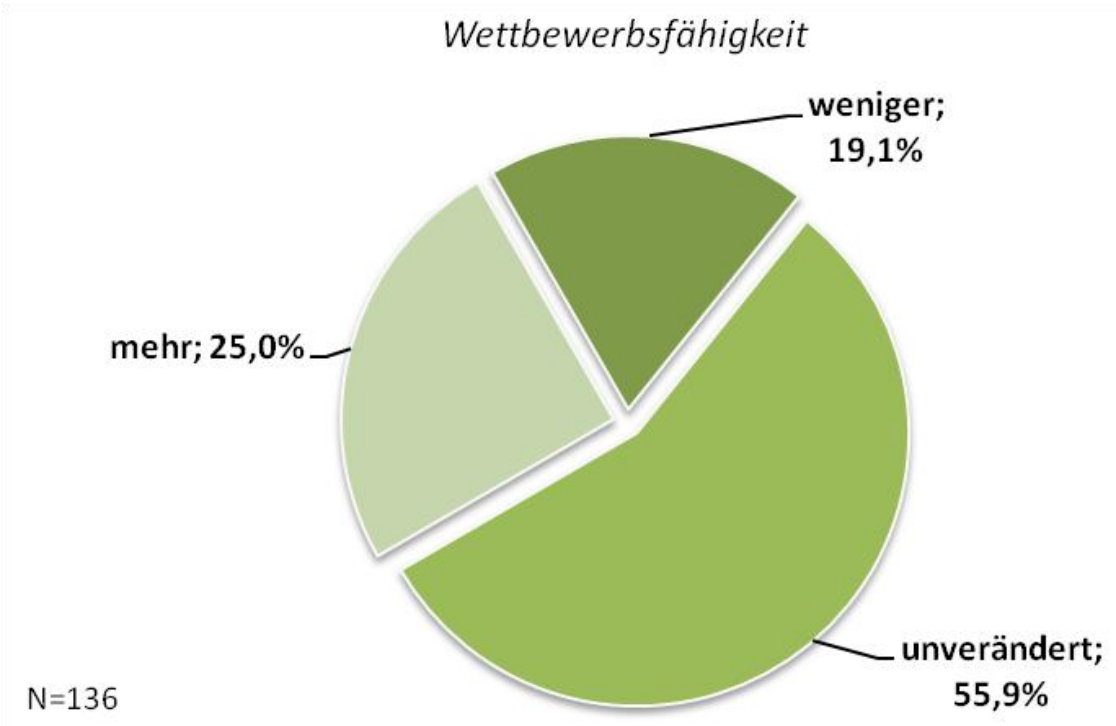




\section{Gender}

Die Unterschiede in der Einschätzung zwischen Frauen und Männern sind gering. Frauen schätzten die Veränderung der Wettbewerbsfähigkeit etwas positiver ein als Männer.

Abbildung 31: Veränderungen bzgl. Wettbewerbsfähigkeit seit 2007 - Gender

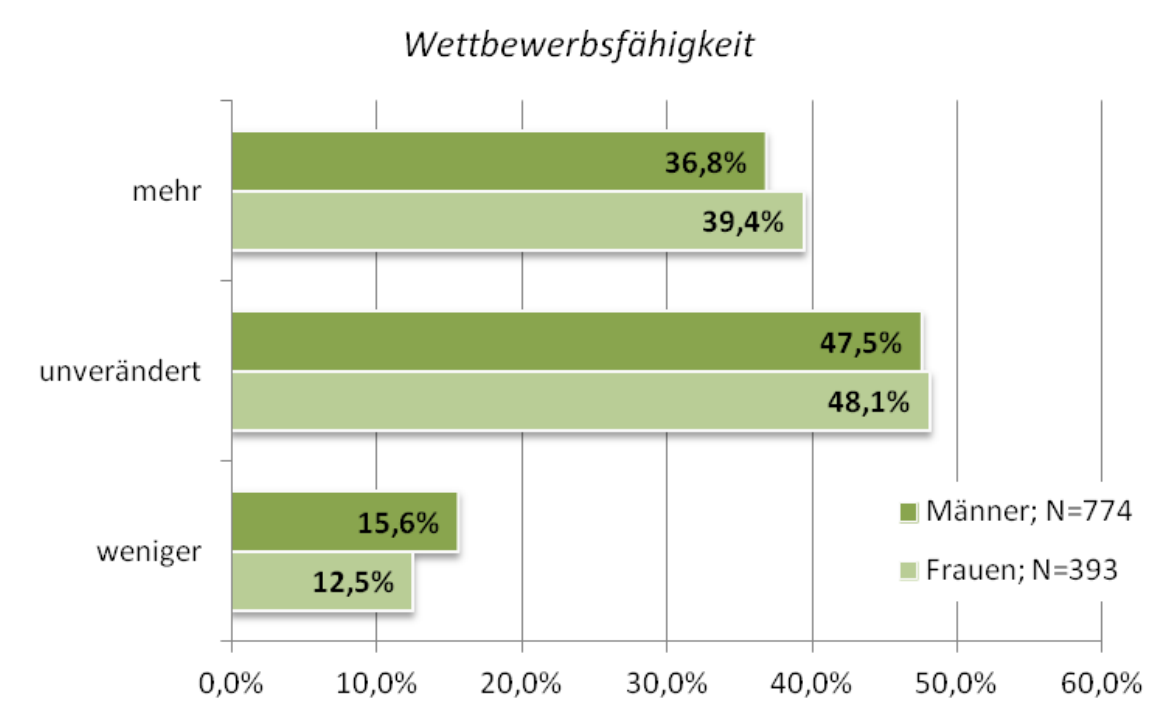

\subsection{Nachhaltiges Flächenmanagement und nachhaltiges Management natürlicher Ressourcen}

Die Wirkungen der Bildungsmaßnahmen der LE im Bereich "Nachhaltiges Flächenmanagement und Nachhaltiges Management natürlicher Ressourcen" wird im Folgenden anhand der Bewertungen in den Kategorien "Nachhaltige Wirtschaftsweise", "Naturschutz- und umweltrelevantes Denken und Handeln“ und „Erzeugung und Nutzung erneuerbarer Energie“ betrachtet.

\section{Bildungsmaßnahmen M 111 \& M 331}

Die Mehrheit dieser Personen schätzt, wie aus den Abbildungen 32-34 hervorgeht, die Situation in den drei angeführten Kategorien als seit 2007 unverändert ein, allerdings sieht eine ebenfalls große Gruppe eine Veränderung in Richtung „mehr".

Abbildung 32: Veränderungen bzgl. nachhaltiger Wirtschaftsweise seit 2007 - M 111 \& M 331

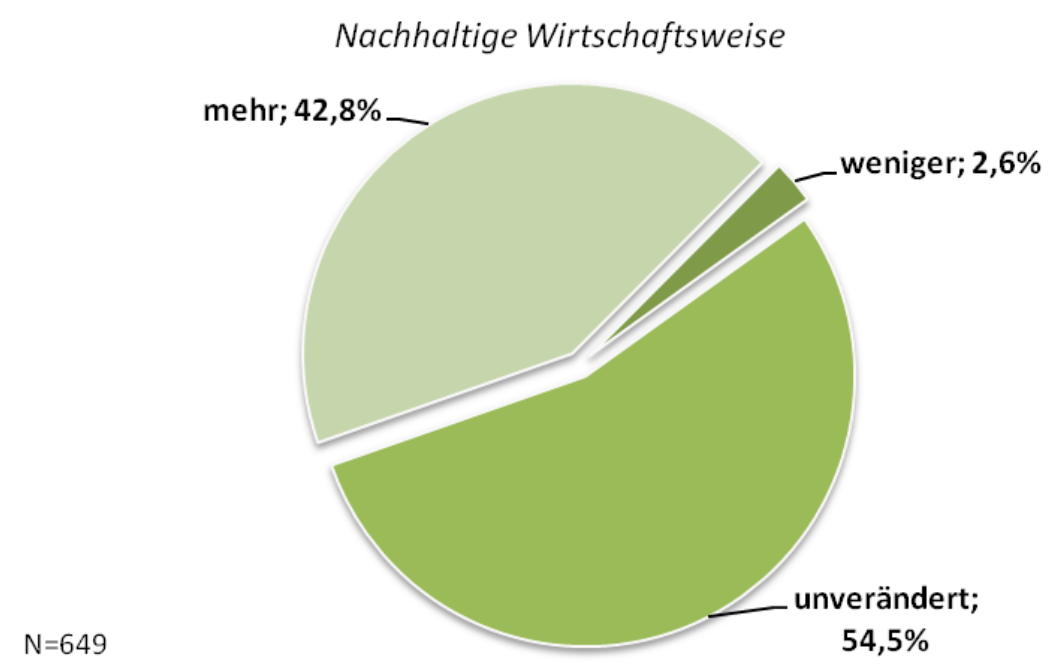


Abbildung 33: Veränderungen bzgl. Naturschutz- und umweltrelevantem Denken und Handeln seit 2007 M 111 \& M 331

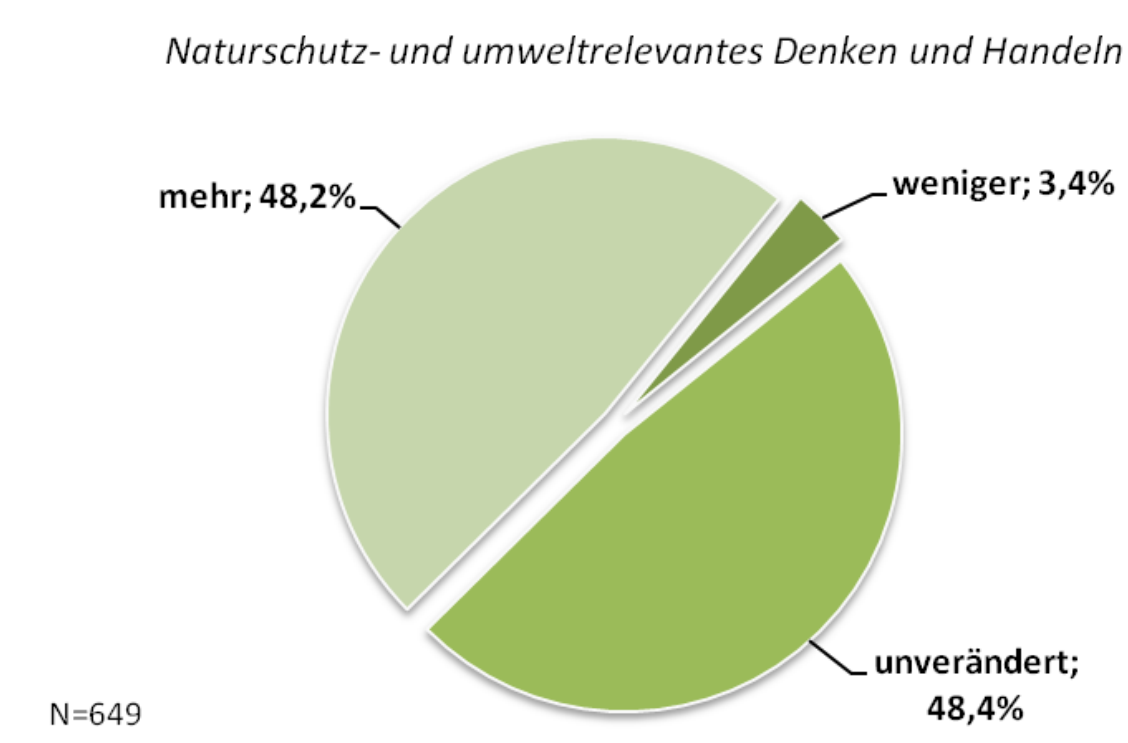

Abbildung 34: Veränderungen bzgl. erneuerbarer Energie seit 2007 - M 111 \& M 331

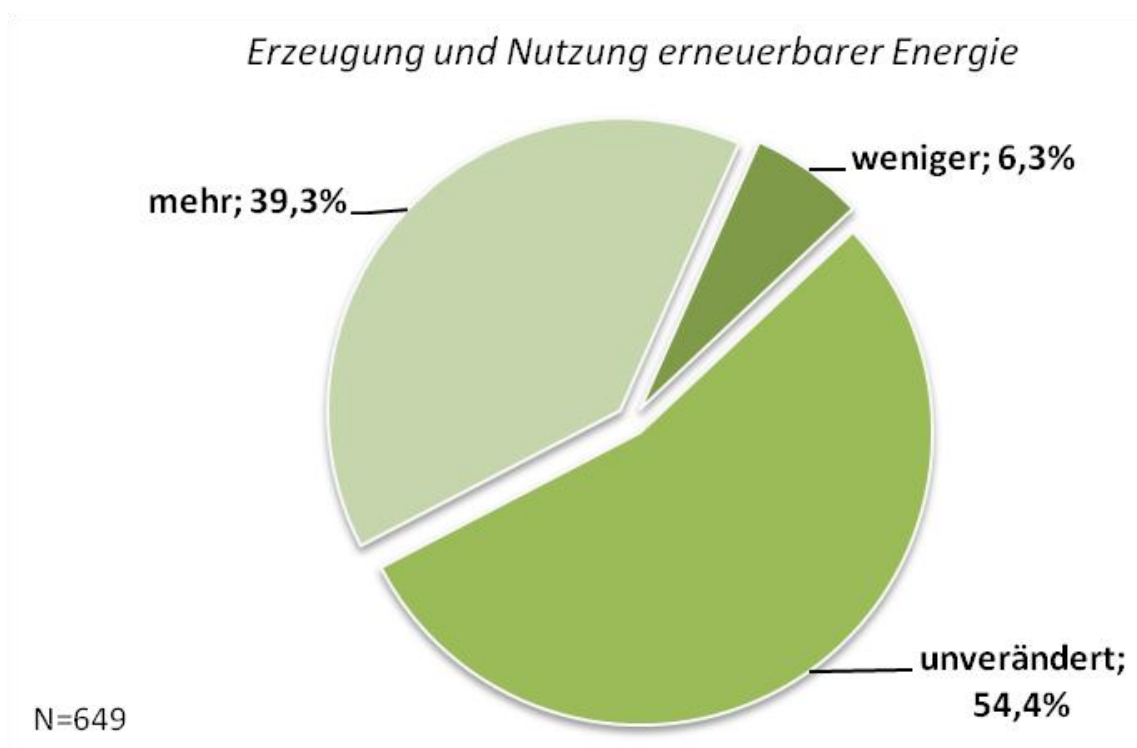




\section{Agrar-Umwelt und Naturschutz}

Für diese Gruppe gab es seit 2007 starke Veränderungen zu einer nachhaltigen Wirtschaftsweise.

Abbildung 35: Veränderungen bzgl. nachhaltiger Wirtschaftsweise seit 2007 - Agrar- Umwelt und Naturschutz

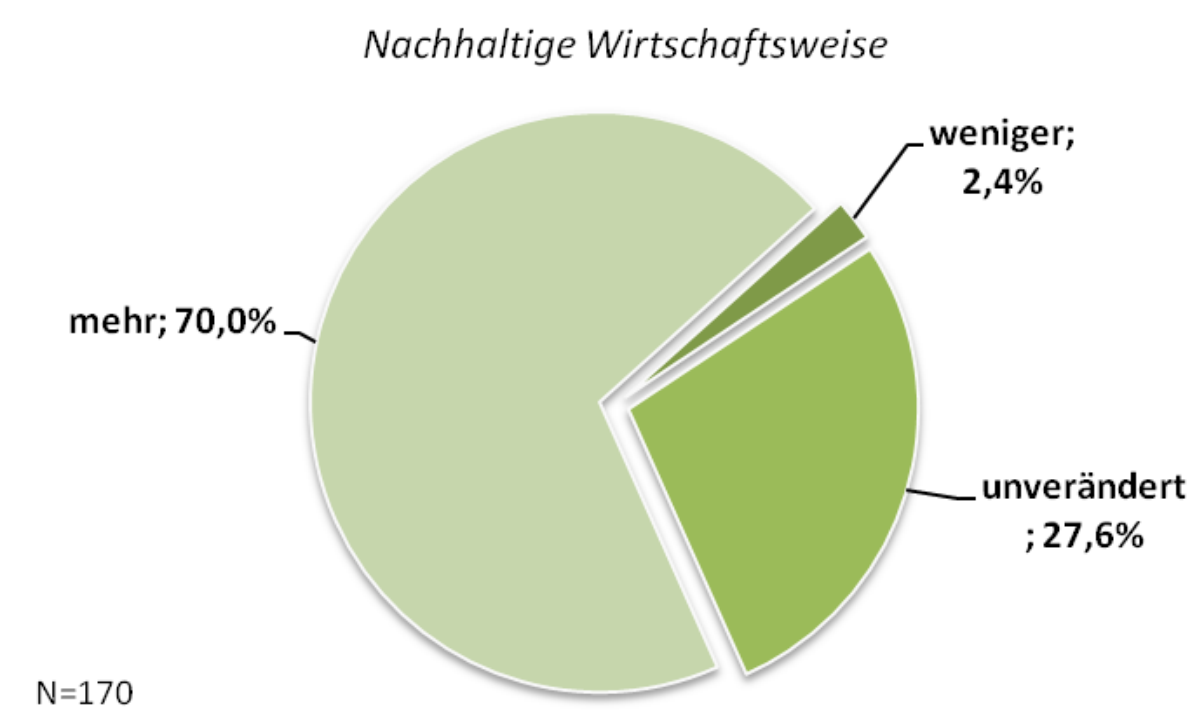

Fast drei Viertel der Befragten in dieser Gruppe sieht zudem seit 2007 eine Steigerung im persönlichen Naturschutz- und umweltrelevanten Denken und Handeln.

Abbildung 36: Veränderungen bzgl. Naturschutz- und umweltrelevantem Denken und Handeln seit 2007 Agrar- Umwelt und Naturschutz

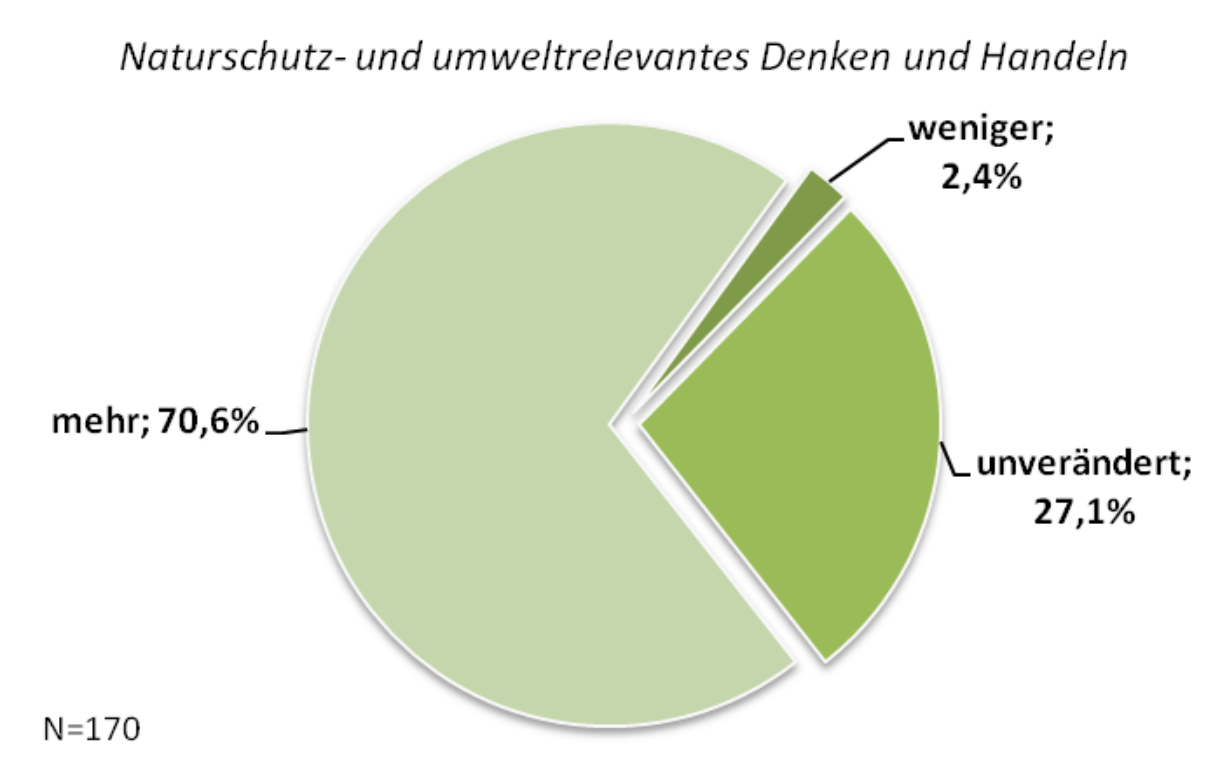

Auch hinsichtlich „Erzeugung und Nutzung erneuerbarer Energie“ wählten mehr als 50\% der Befragten die Kategorie "mehr". 
Abbildung 37: Veränderungen bzgl. erneuerbarer Energie seit 2007 - Agrar- Umwelt und Naturschutz

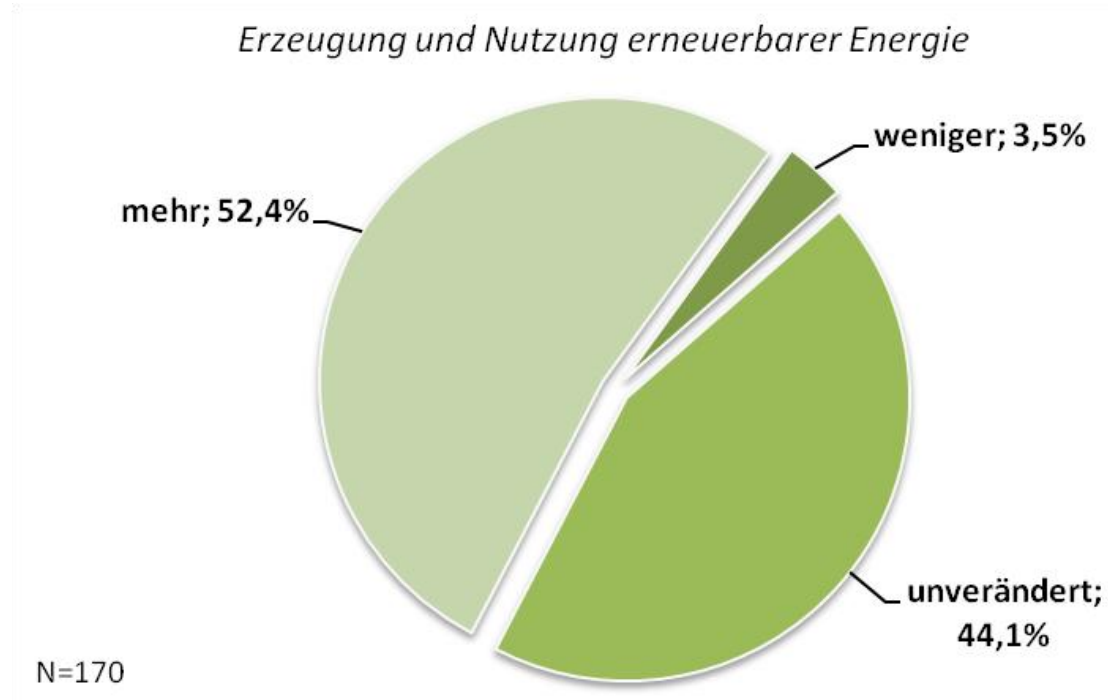

\section{Forstbildungsmaßnahmen}

Auch bei den Personen, die forstwirtschaftliche Kurse besuchten, liegen die Einschätzungen in allen drei Bereichen zum Großteil in den Bewertungskategorien „mehr" und „unverändert".

Abbildung 38: Veränderungen bzgl. nachhaltiger Wirtschaftsweise seit 2007 - Forstbildungsmaßnahmen

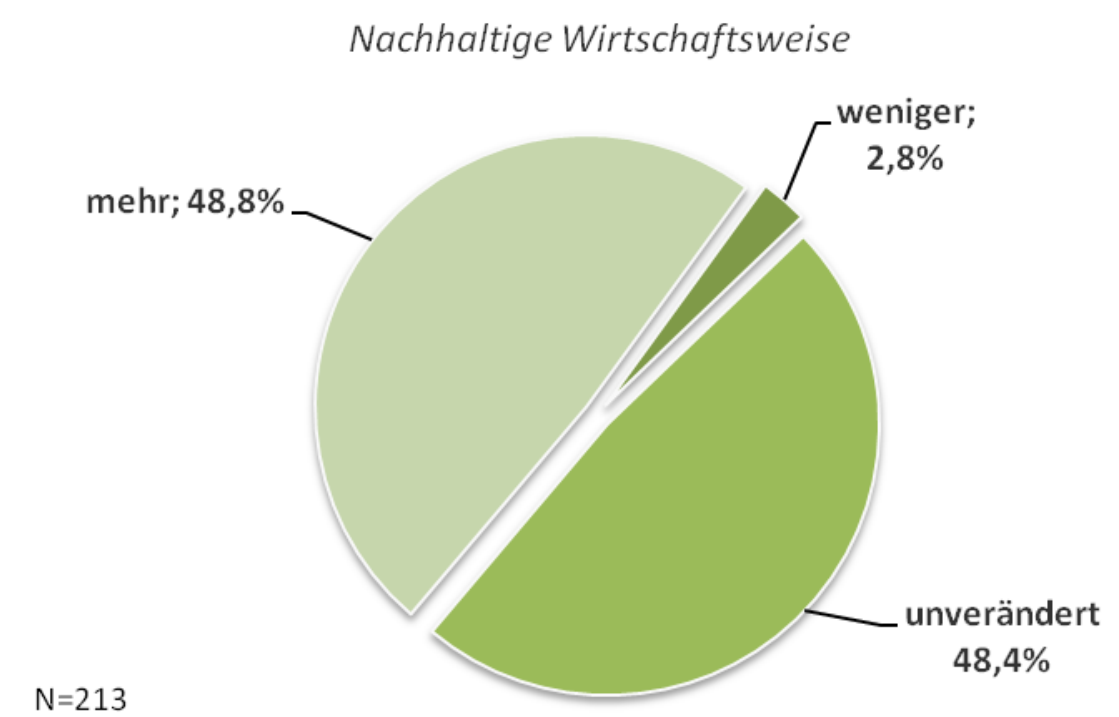


Abbildung 39: Veränderungen bzgl. Naturschutz- und umweltrelevantem Denken und Handeln seit 2007 Forstbildungsmaßnahmen

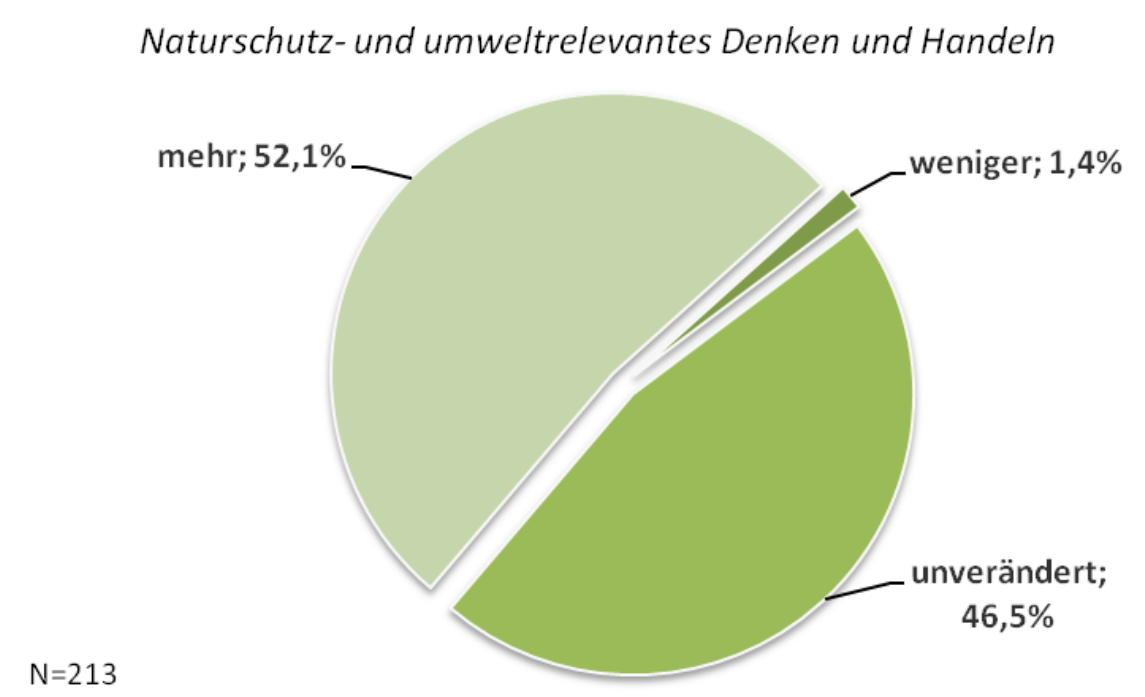

Abbildung 40: Veränderungen bzgl. erneuerbarer Energie seit 2007 - Forstbildungsmaßnahmen

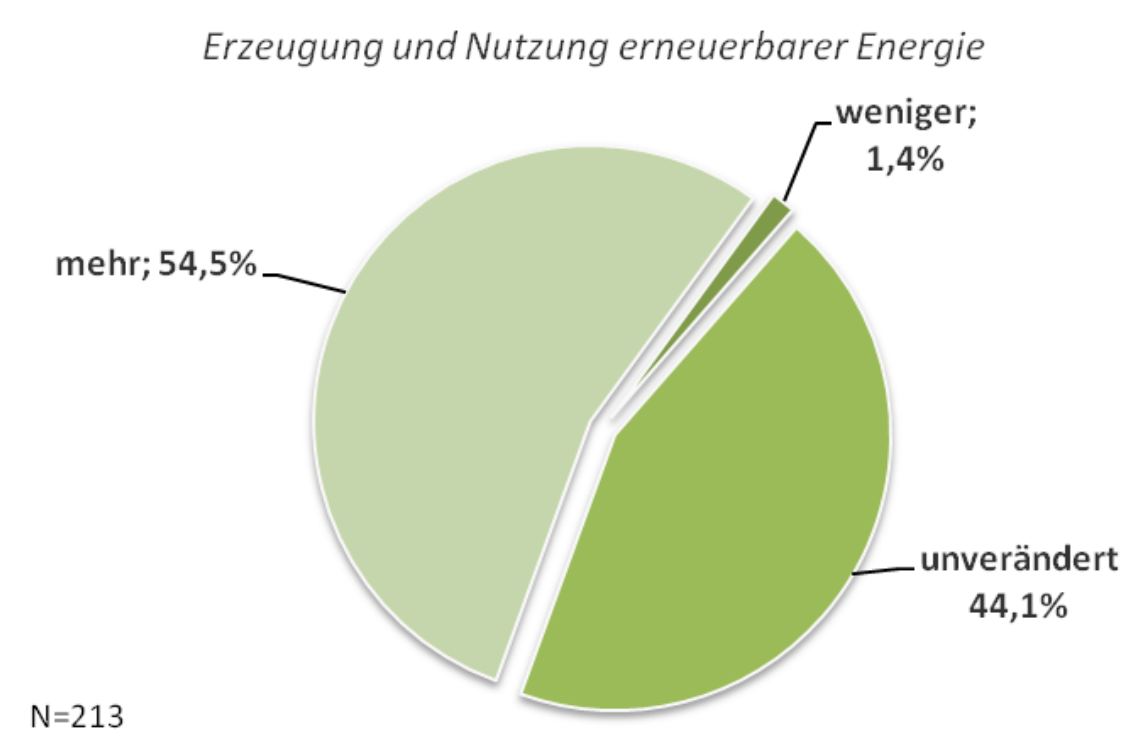

Dies passt gut überein mit den folgenden Aussagen, die Bezüglich der Wirkung der Bildungsmaßnahmen im Bereich Forst in den Dialog-Interviews gemacht wurden:

„Der Wald wird besser bewirtschaftet - ökonomisch und ökologisch."

„Mögliche Effekte sind extrem langfristig, weil Wald generell nur sehr langfristig Wirkungen zeigt. Eine Generation trifft Entscheidungen und setzt Maßnahmen, aber erst die nächste Generation kann sagen, ob es gute Entscheidungen waren und die Maßnahmen sinnvoll gesetzt wurden." 


\section{Land-bzw. ForstwirtInnen, die keine Bildungsangebote besucht haben}

Im Vergleich zwischen Land- bzw. ForstwirtInnen, die seit 2007 Bildungsangebote der LE besucht haben mit jenen, die keine Kurse besuchten zeigt sich, dass besonders in den Bereichen „Nachhaltige Wirtschaftsweise" und "Naturschutz- und umweltrelevantes Denken und Handeln" für Erstere ein größerer Anstieg in beiden Bereichen feststellbar ist.

Abbildung 41: Nachhaltige Wirtschaftsweise - Vergleich

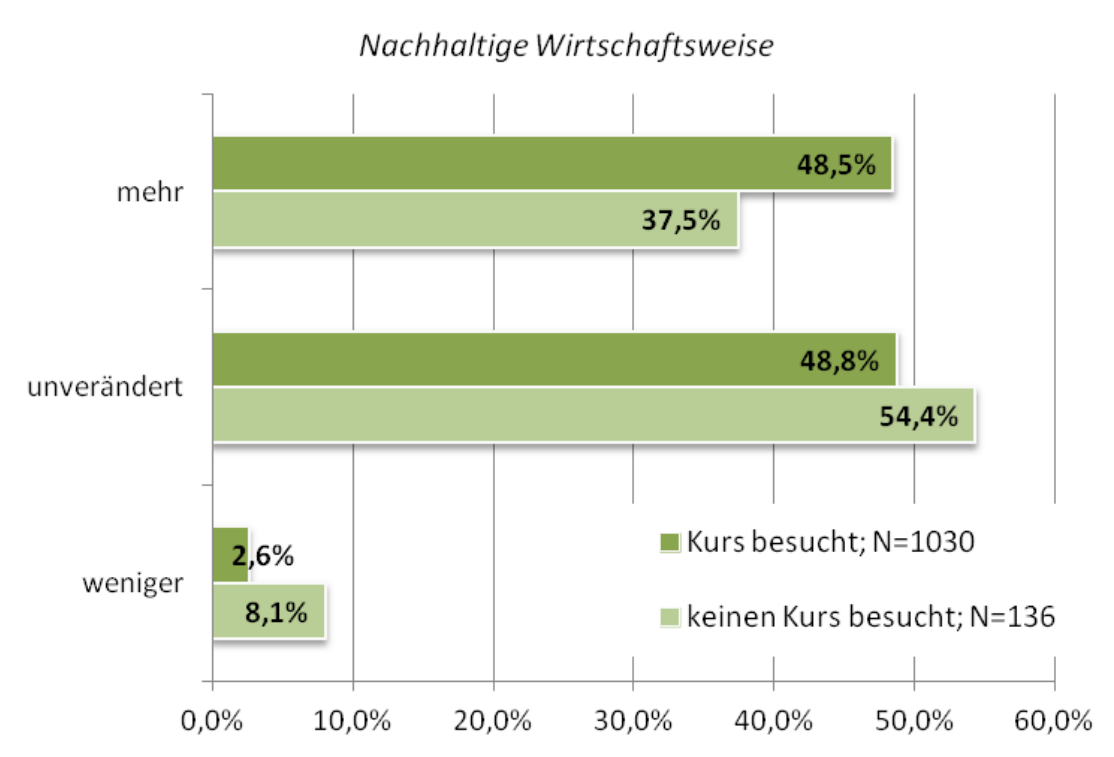

Abbildung 42: Naturschutz- und umweltrelevantem Denken und Handeln - Vergleich

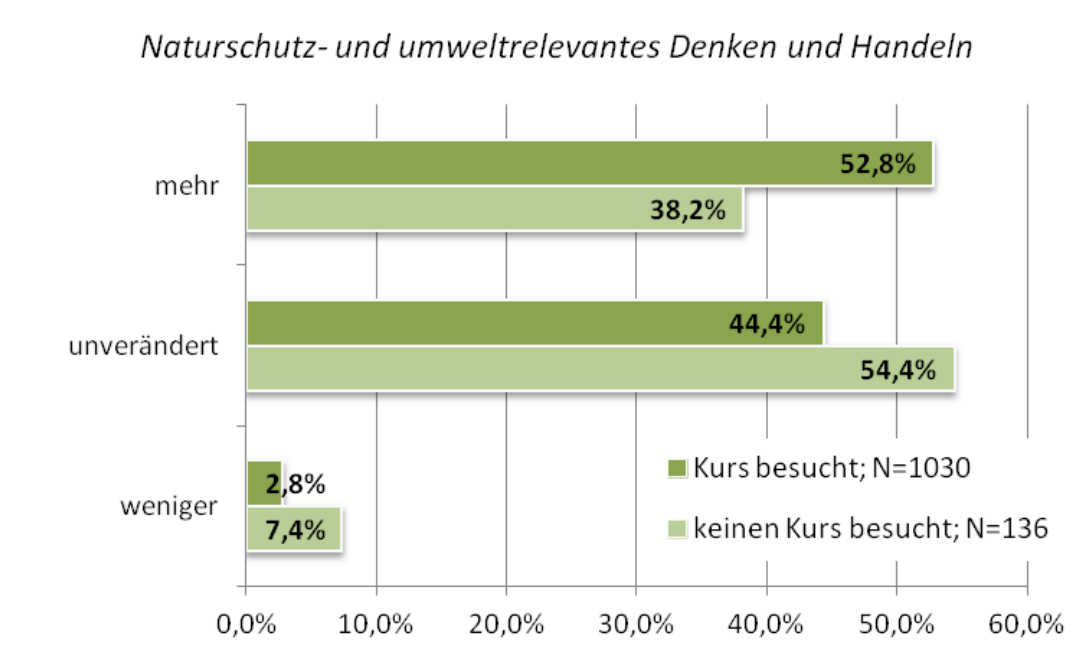

Die Wahrnehmung bzgl. der Veränderung in der Erzeugung und Nutzung erneuerbarer Energie ist bei beiden Gruppen ähnlich. Die meisten Antworten wurden hier in der Kategorie "unverändert" gegeben. 
Abbildung 43: Erzeugung und Nutzung erneuerbarer Energie - Vergleich

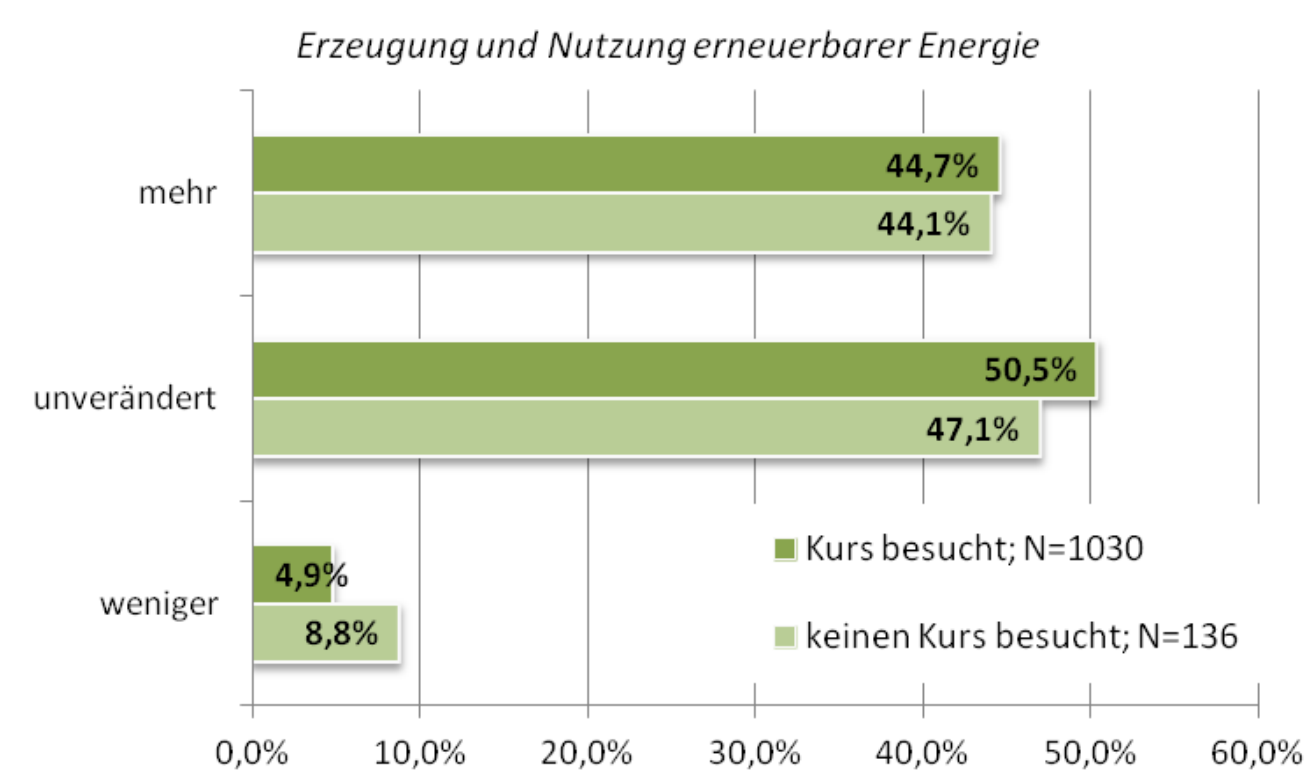

\section{Gender}

In den Bereichen "Nachhaltige Wirtschaftsweise" und "Naturschutz- und umweltrelevantes Denken und Handeln" schätzten die teilnehmenden Frauen die Veränderung mehrheitlich als Steigerung ein, während Männer dies nur in letzterem Bereich mehrheitlich so einschätzen.

Abbildung 44: Veränderungen bzgl. nachhaltiger Wirtschaftsweise seit 2007 - Gender

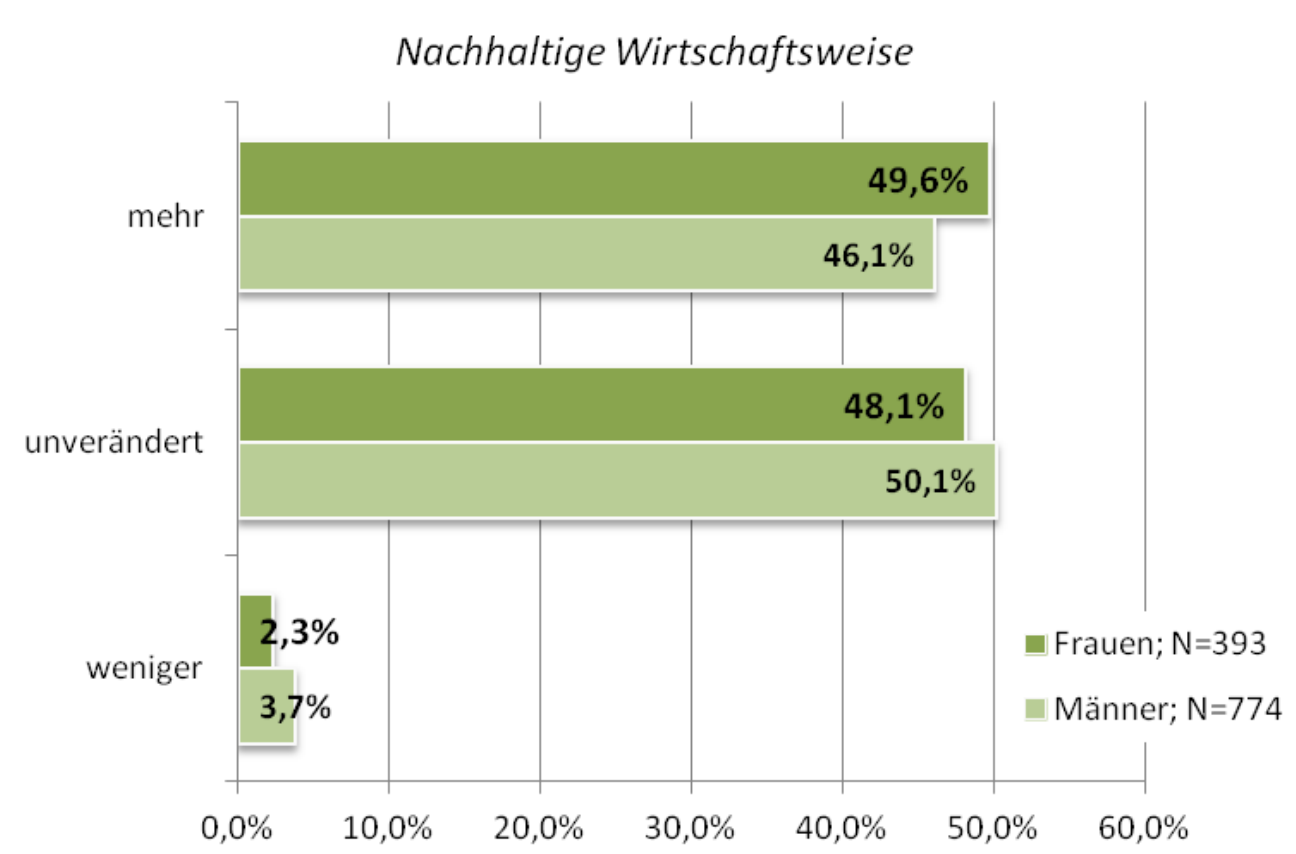


Abbildung 45: Veränderungen bzgl. Naturschutz- und umweltrelevantem Denken und Handeln seit 2007 Gender

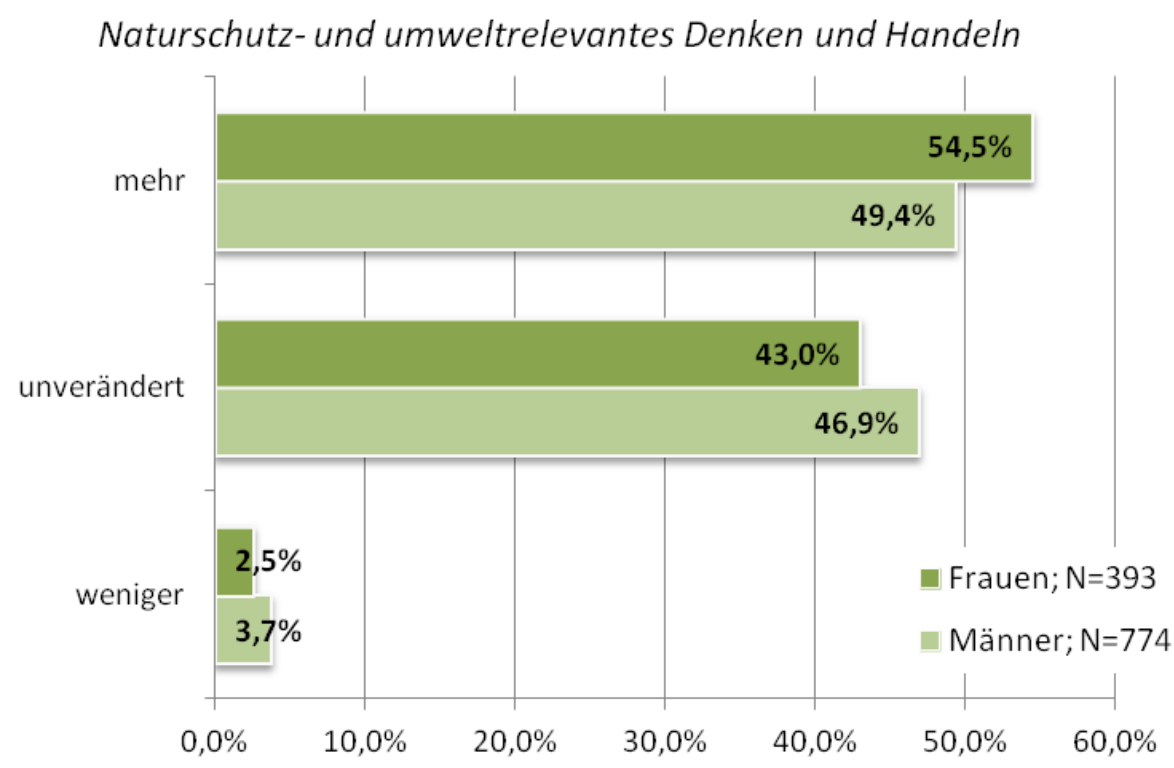

Ein signifikanter Unterschied zwischen Frauen und Männern besteht bei der Einschätzung bzw. Wahrnehmung der Veränderung bzgl. erneuerbarer Energie. Während Frauen die Situation mehrheitlich klar mit "unverändert" beurteilten, liegen bei den Männern die Antwortzahlen von "mehr" und "unverändert" doch deutlich näher beisammen.

Abbildung 46: Veränderungen bzgl. erneuerbarer Energie seit 2007 - Gender

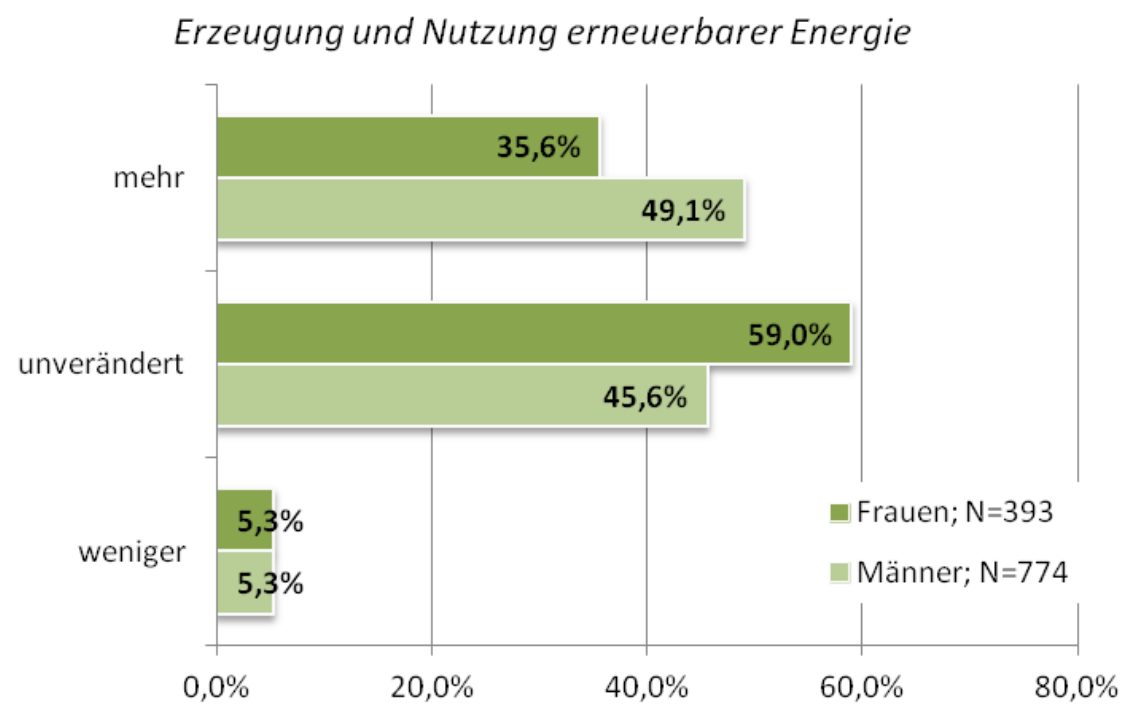




\subsection{Bedarf an Bildungsmaßnahmen und Kohärenz mit anderen Maßnahmen des Programms}

Der Bedarf bzw. die Nachfrage seitens der Land- bzw. ForstwirtInnen nach (Aus-) Bildungs- und Informationsangeboten wird in diesem Kapitel anhand zweier Fragen aus der Online-Umfrage analysiert. Im Fragebogen wurden die TeilnehmerInnen dazu einerseits gebeten anzugeben, zu welchen Themen sie selbst seit 2007 Kurse der LE besucht haben, andererseits sollten sie bewerten, wie die verschiedenen Themen aus ihrer Sicht im Bildungsangebot vertreten sind.

In den Dialog-Interviews wurden zum Thema „Bedarf“ folgende Aussagen getätigt:

„Geplant waren 60 Veranstaltungen, tatsächlich wurden jedoch nur 40 durchgeführt, weil der Bedarf nicht so groß war bzw. es zu viele andere Bildungsangebote gleichzeitig gab."

„Das Bildungsangebot im Rahmen der verpflichtenden Weiterbildung für Schule am BauernhofBetreiberInnen ist nicht 100\% interessant, vor allem wenn man schon die meisten Kurse besucht hat. Wünschenswert wäre, dass man auch die Angebote anderer Veranstalter außerhalb des Schule am Bauernhof Programms nutzen kann und gefördert bekommt."

"Die Nachfrage sollte geprüft werden, denn es besteht ein Überangebot an Kursen."

„Es wird zu wenig Persönlichkeitsbildung angeboten."

Nachfolgend werden die Auswertungen der Online-Befragung getrennt nach den Bildungsmaßnahmen betrachtet.

Bildungsmaßnahmen M 111 \& M 331

Bei der Bewertung des Bildungsangebotes wurden in der Gesamtbetrachtung von den 15 aufgelisteten Themen (siehe z.B. Abbildung 47a) 13 mehrheitlich mit "gerade richtig" bewertet. Bei „Erhaltung und Verbesserung des ländlichen Erbes (Kulturlandschaft,...)" und "Kommunale und soziale Dienstleistungen, Dorferneuerung und Dorfentwicklung" wurde allerdings mehrheitlich jeweils die Option "keine Angabe“ (nicht in der Abbildung dargestellt) gewählt. 
Abbildung 47a: Bewertung des Bildungsangebotes (absolut) - M 111 \& M 331

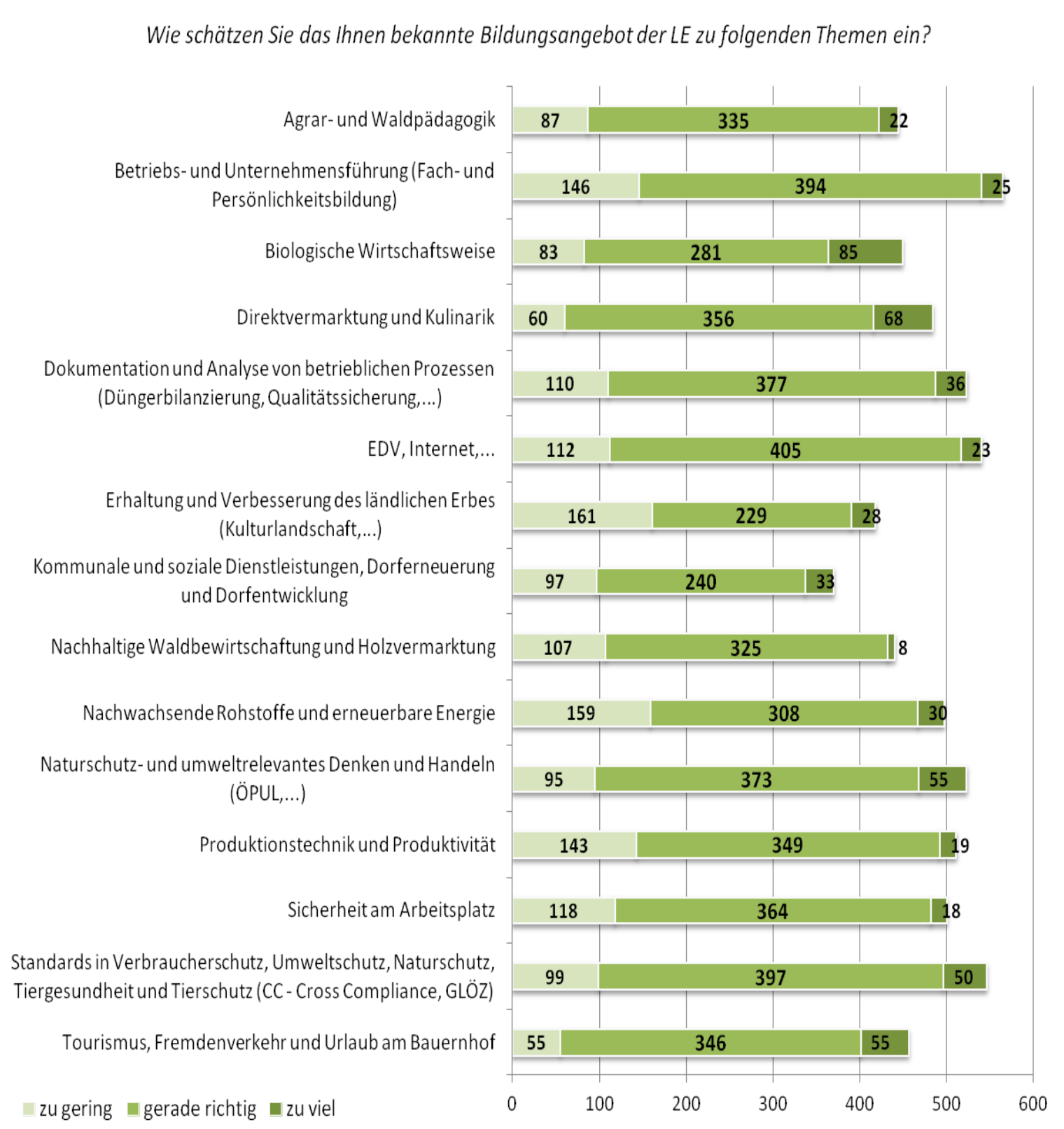


Abbildung 47b: Bewertung des Bildungsangebotes (in \%) - M 111 \& M 331

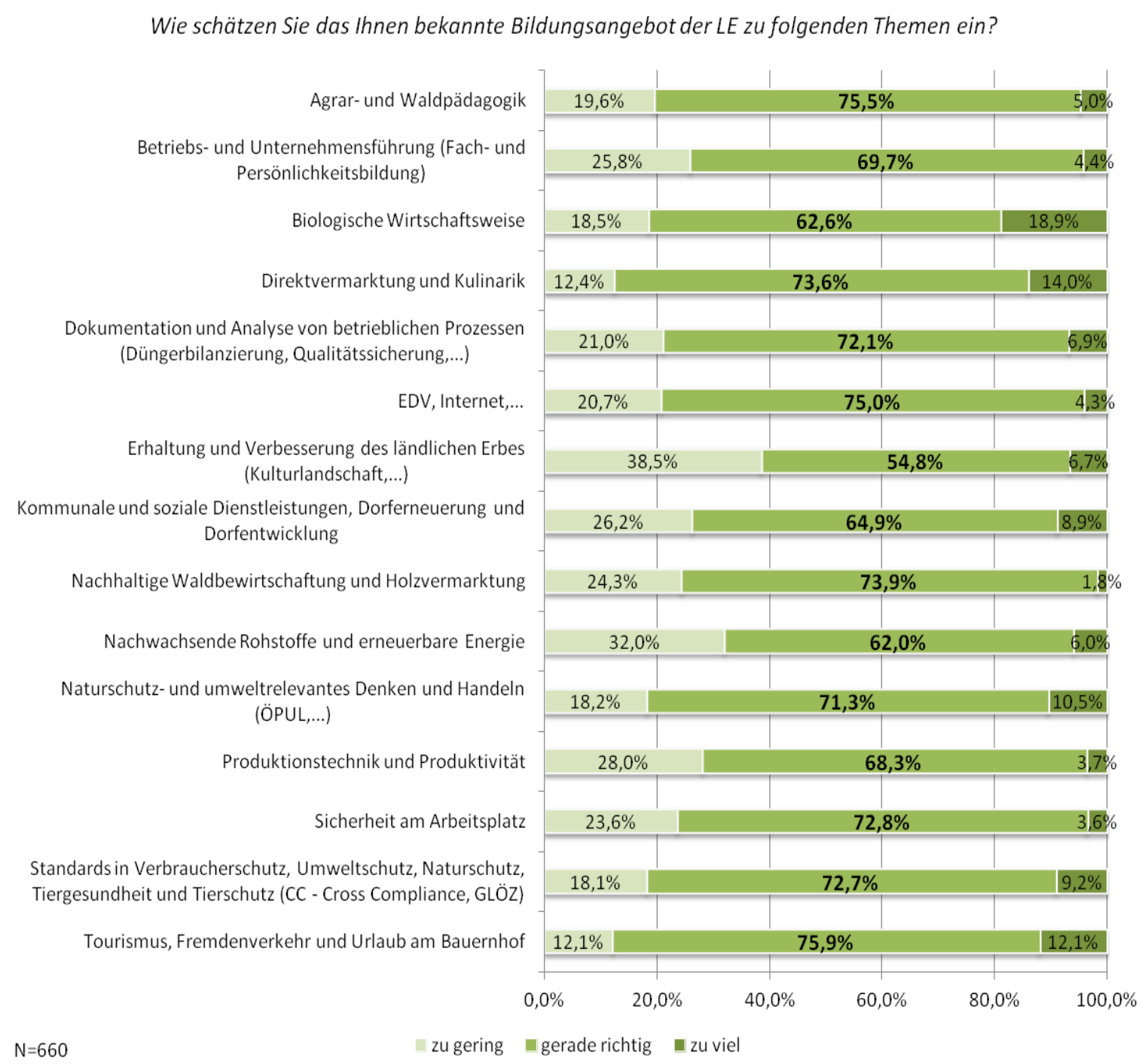

Die meisten der befragten Personen aus dieser Gruppe gaben an, Kurse zu „Betriebs- und Unternehmensführung (Fach- und Persönlichkeitsbildung)“ gewählt zu haben. An zweiter und dritter Stelle wurden Kurse zu "Standards in Verbraucherschutz, etc. " und "Produktionstechnik und Produktivität" absolviert. Bei dieser Frage konnten mehrere Antworten gewählt werden.

Wie zu Beginn des Kapitels 4 erwähnt, erfolgte für die Auswertungen die Einteilung in die hier und in den vorhergehenden Kapiteln betrachteten Gruppen (Bildungsmaßnahmen M 111 / M 331; Agrar-Umwelt und Naturschutz; Forstbildungsmaßnahmen) anhand der, in der folgenden Abbildung 48 dargestellten Frage aus der Online-Befragung. Die Gruppe „Bildungsmaßnahmen M 111 \& M 331" beinhaltet keine Personen, die angaben, Kurse zu „Biologische Wirtschaftsweise“ und "Nachhaltige Waldbewirtschaftung und Holzvermarktung" absolviert zu haben, was in der Abbildung ersichtlich ist. Diese beiden Themenfelder werden in den Gruppen „Agrar-Umwelt und Naturschutz" und „Forstbildungsmaßnahmen“ abgedeckt (siehe Abbildungen 50 und 52). 
Abbildung 48: Besuchte Kurse - M 111 \& M 331

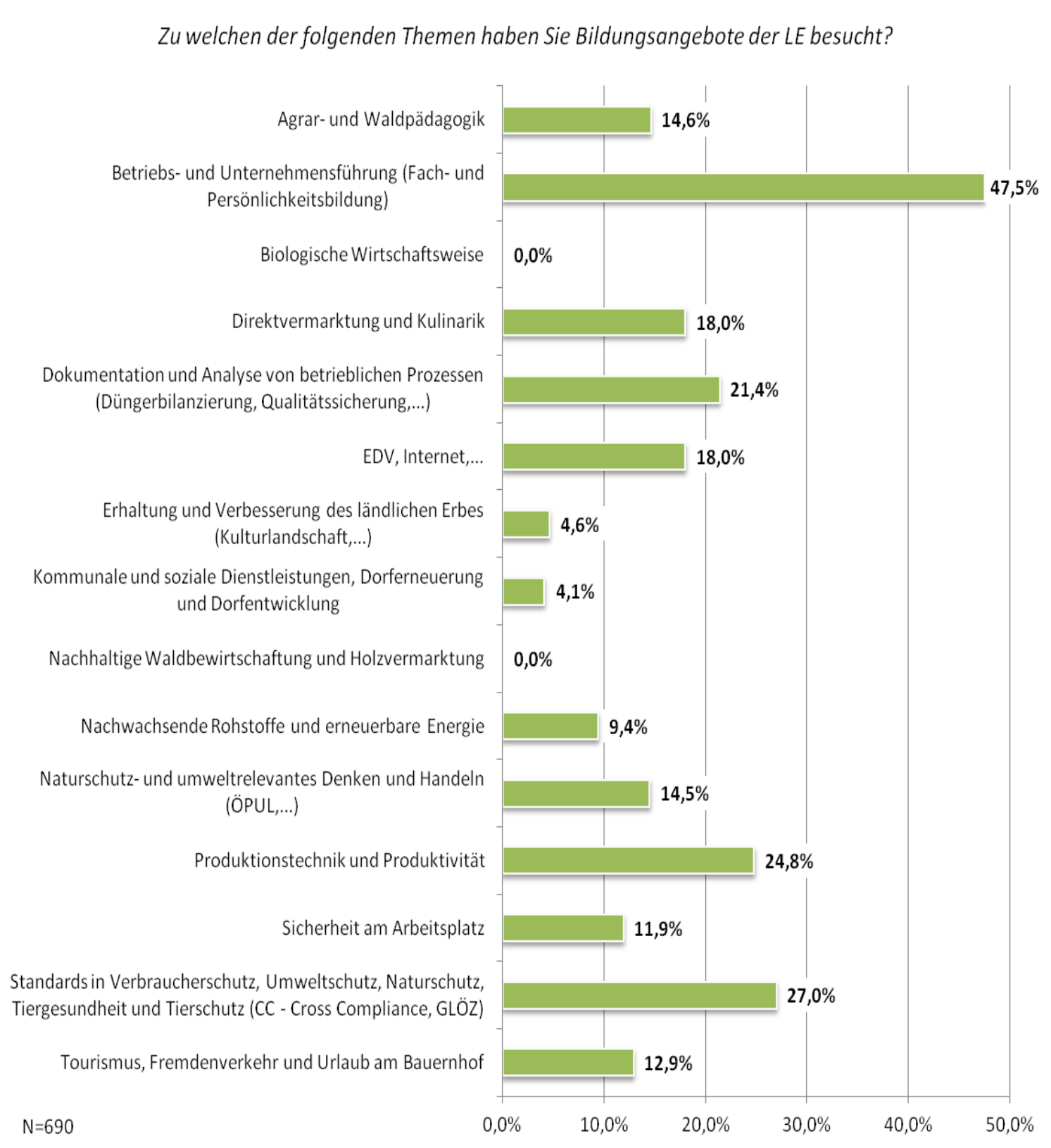




\section{Agrar-Umwelt und Naturschutz}

Hier fällt bei der Betrachtung der Auswertungen zur Einschätzung des Bildungsangebotes auf, dass in den Bereichen "Biologische Wirtschaftsweise", "Erhaltung und Verbesserung des ländlichen Erbes (Kulturlandschaft,...)", "Nachwachsende Rohstoffe und erneuerbare Energie" und "Naturschutz- und umweltrelevantes Denken und Handeln" jeweils der Anteil des Bewertungskriteriums „zu wenig“ relativ groß ist (über 30\%).

Abbildung 49a: Bewertung des Bildungsangebotes (absolut) - Agrar-Umwelt und Naturschutz

Wie schätzen Sie das Ihnen bekannte Bildungsangebot der LE zu folgenden Themen ein?

Dokumentation und Analyse von betrieblichen Prozessen (Düngerbilanzierung, Qualitätssicherung,...)

EDV, Internet,...

Erhaltung und Verbesserung des ländlichen Erbes (Kulturlandschaft,...)

Kommunale und soziale Dienstleistungen, Dorferneuerung und Dorfentwicklung

Nachhaltige Waldbewirtschaftung und Holzvermarktung

Nachwachsende Rohstoffe und erneuerbare Energie

Naturschutz- und umweltrelevantes Denken und Handeln (ÖPUL,...)

Produktionstechnik und Produktivität

Sicherheit am Arbeitsplatz

Standards in Verbraucherschutz, Umweltschutz, Naturschutz, Tiergesundheit und Tierschutz (CC - Cross Compliance, GLÖZ)

Tourismus, Fremdenverkehr und Urlaub am Bauernhof
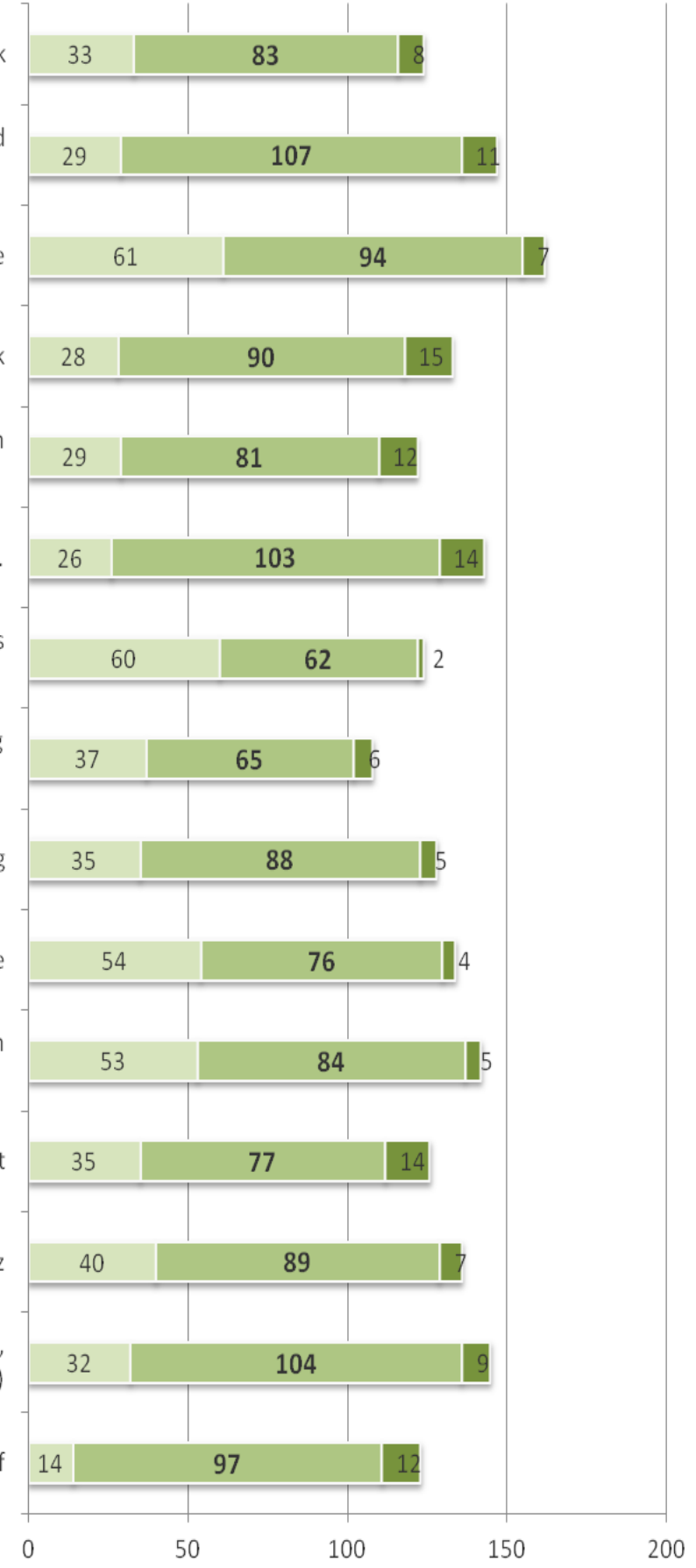


\section{Abbildung 49b: Bewertung des Bildungsangebotes (in \%) - Agrar-Umwelt und Naturschutz}

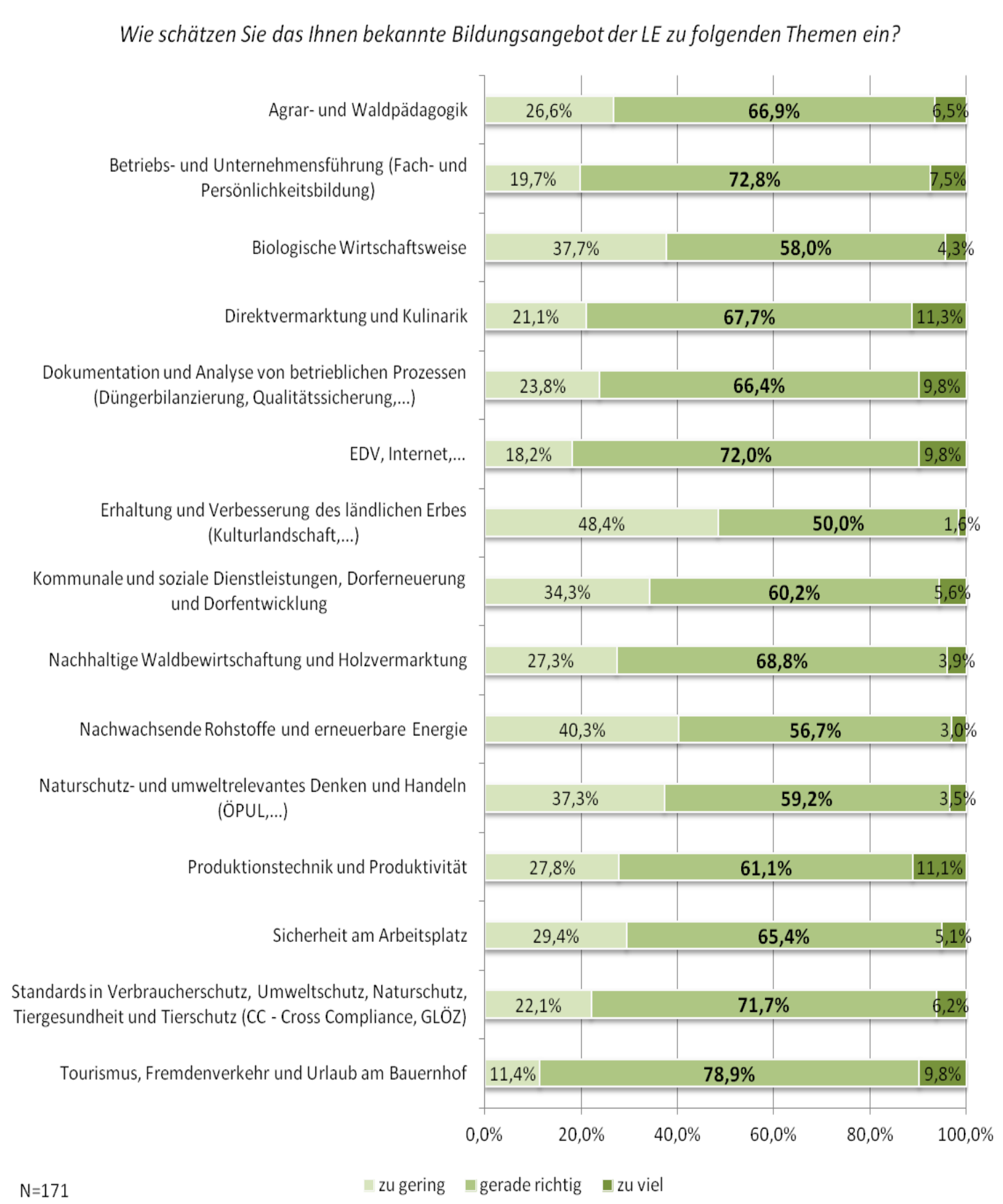


Auch in dieser Gruppe waren, neben Kursen zu biologischer Wirtschaftsweise, jene zu Betriebsführung und Standards am meisten besucht. Deutlich häufiger als in der Gruppe der Bildungsmaßnahmen M 111 \& M 331 wurde hier auch das Thema „Direktvermarktung und Kulinarik" gewählt.

\section{Abbildung 50: Besuchte Kurse - Agrar-Umwelt und Naturschutz}

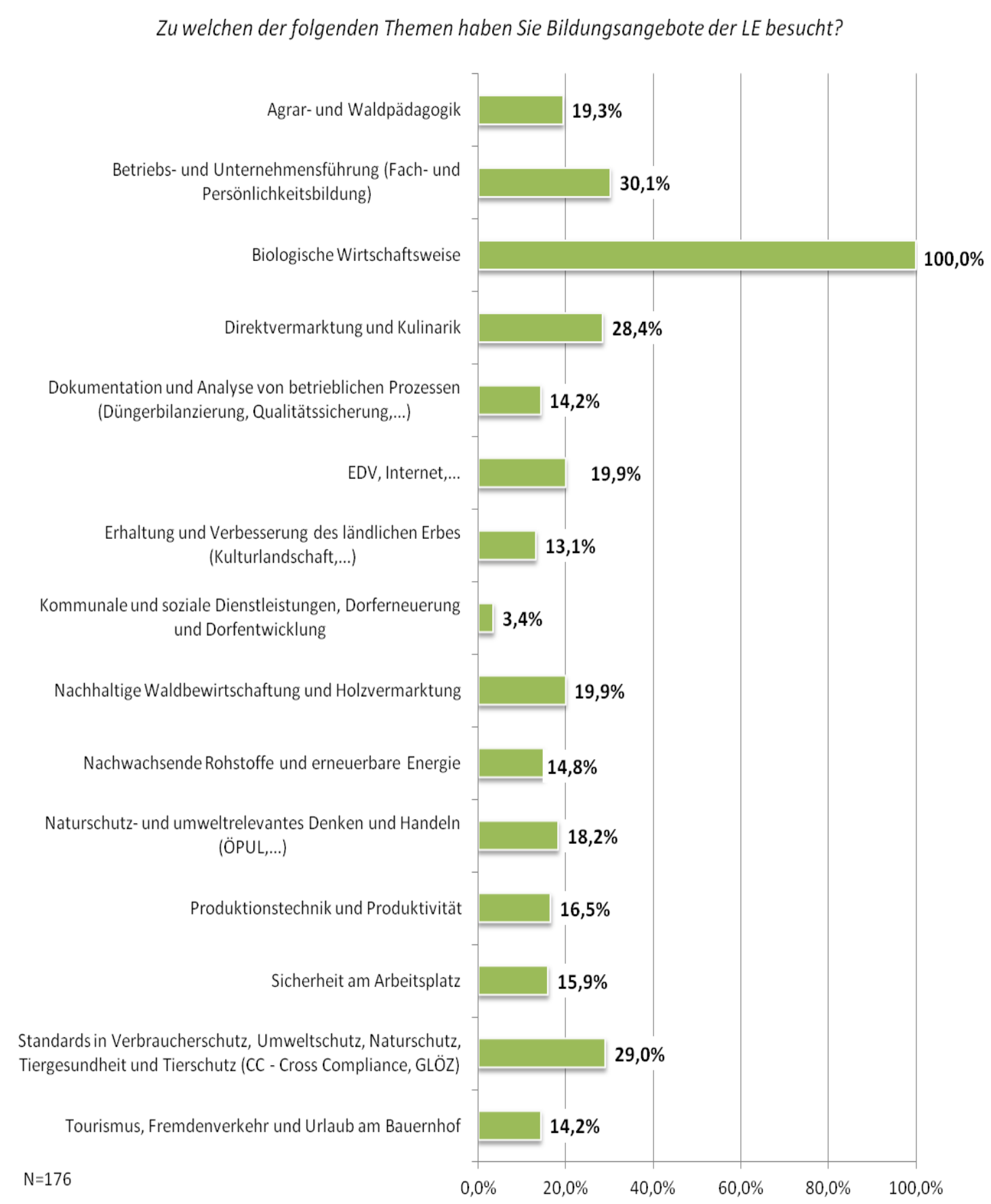




\section{Forstbildungsmaßnahmen}

Personen aus der Land- bzw. Forstwirtschaft, die seit 2007 an Forstbildungsmaßnahmen teilgenommen haben, wählten bei den Themen "Kommunale und soziale Dienstleistungen", "Tourismus, Fremdenverkehr und Urlaub am Bauernhof", "Direktvermarktung und Kulinarik" sowie "Erhaltung und Verbesserung des ländlichen Erbes" und "Biologische Wirtschaftsweise" mehrheitlich jeweils die Option „keine Angabe“ (nicht in der Abbildung dargestellt).

\section{Abbildung 51a: Bewertung des Bildungsangebotes (absolut) - Forstbildungsmaßnahmen}

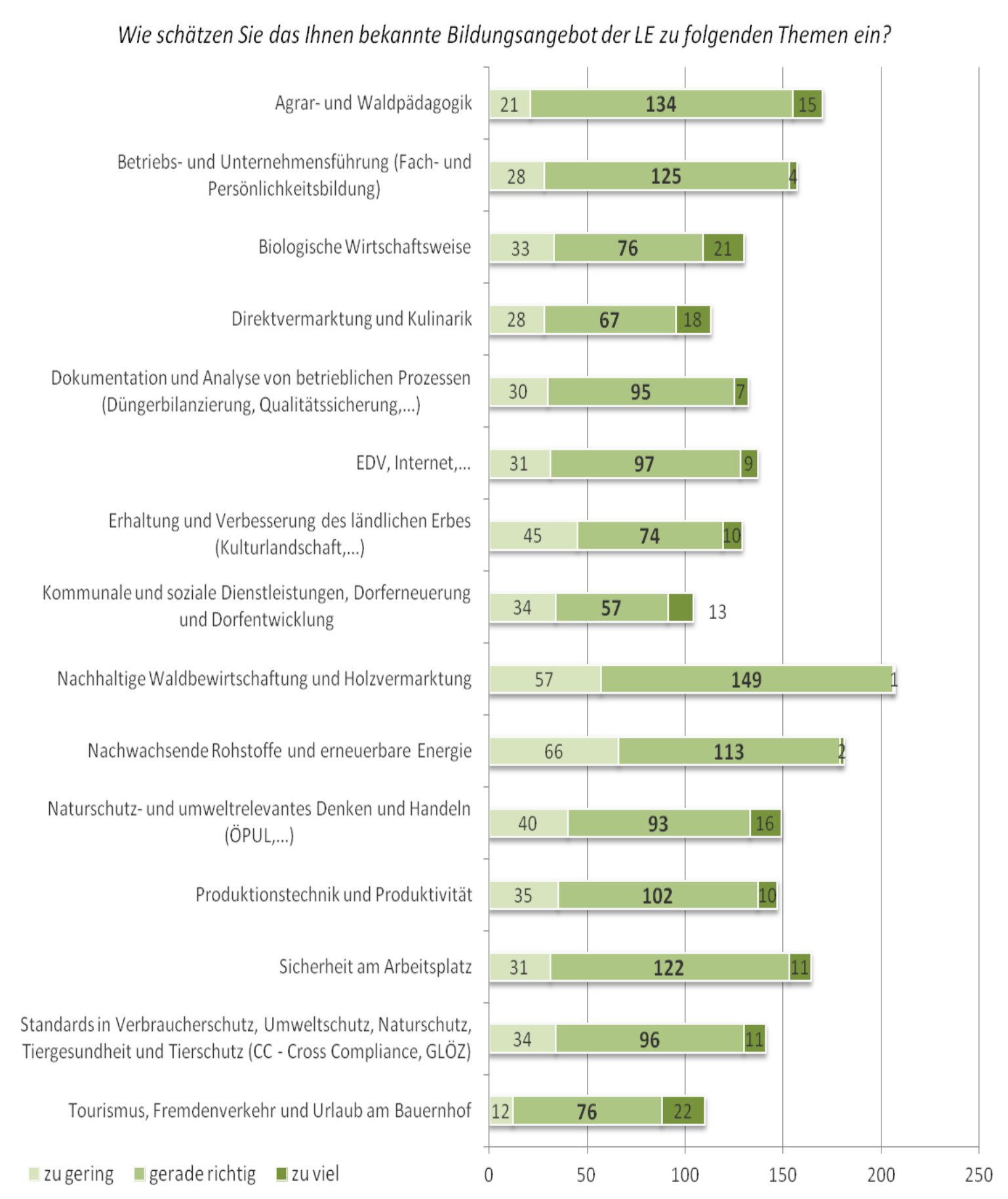


Abbildung 51b: Bewertung des Bildungsangebotes (in \%) - Forstbildungsmaßnahmen

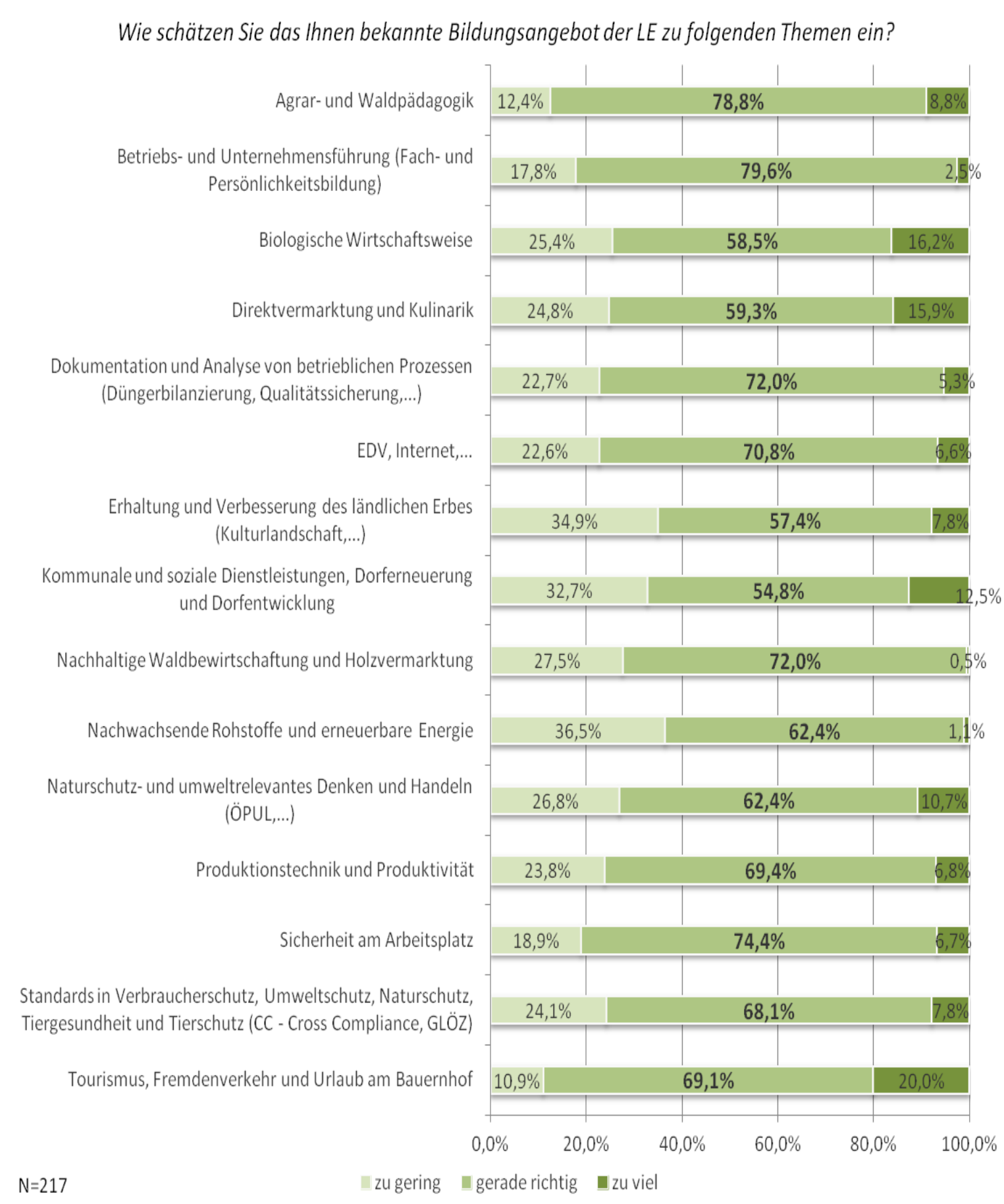


Neben Kursen zu nachhaltiger Waldbewirtschaftung und zu Betriebsführung nutzte diese Personengruppe vor allem auch noch Angebote $\mathrm{zu}$ "Nachwachsende Rohstoffe und erneuerbare Energie".

Abbildung 52: Besuchte Kurse - Forstbildungsmaßnahmen

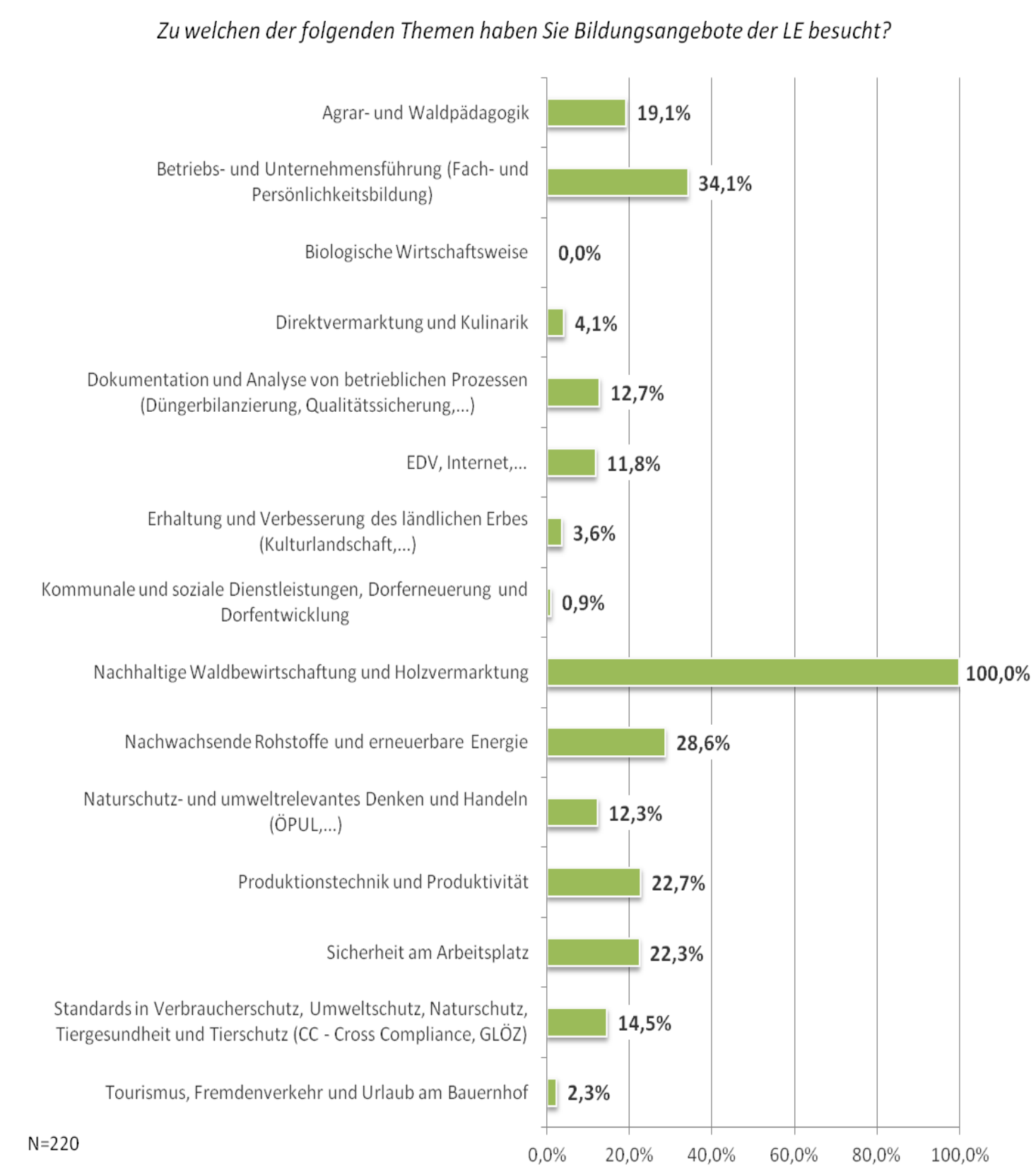

Im Zusammenhang mit dem Bedarf wurden die Land- bzw. ForstwirtInnen auch gefragt, mittels welcher Informationsquellen sie von den Angeboten erfuhren. Bei dieser Frage konnten bis zu drei Antwortmöglichkeiten ausgewählt werden.

Eine Gegenüberstellung der Antworten der Land- bzw. ForstwirtInnen (gesamt) mit jenen der Veranstalter findet sich in Kapitel 4.11.2. 
Abbildung 53: Informationsquellen zu den Bildungsangeboten - Vergleich nach Bildungsmaßnahmen

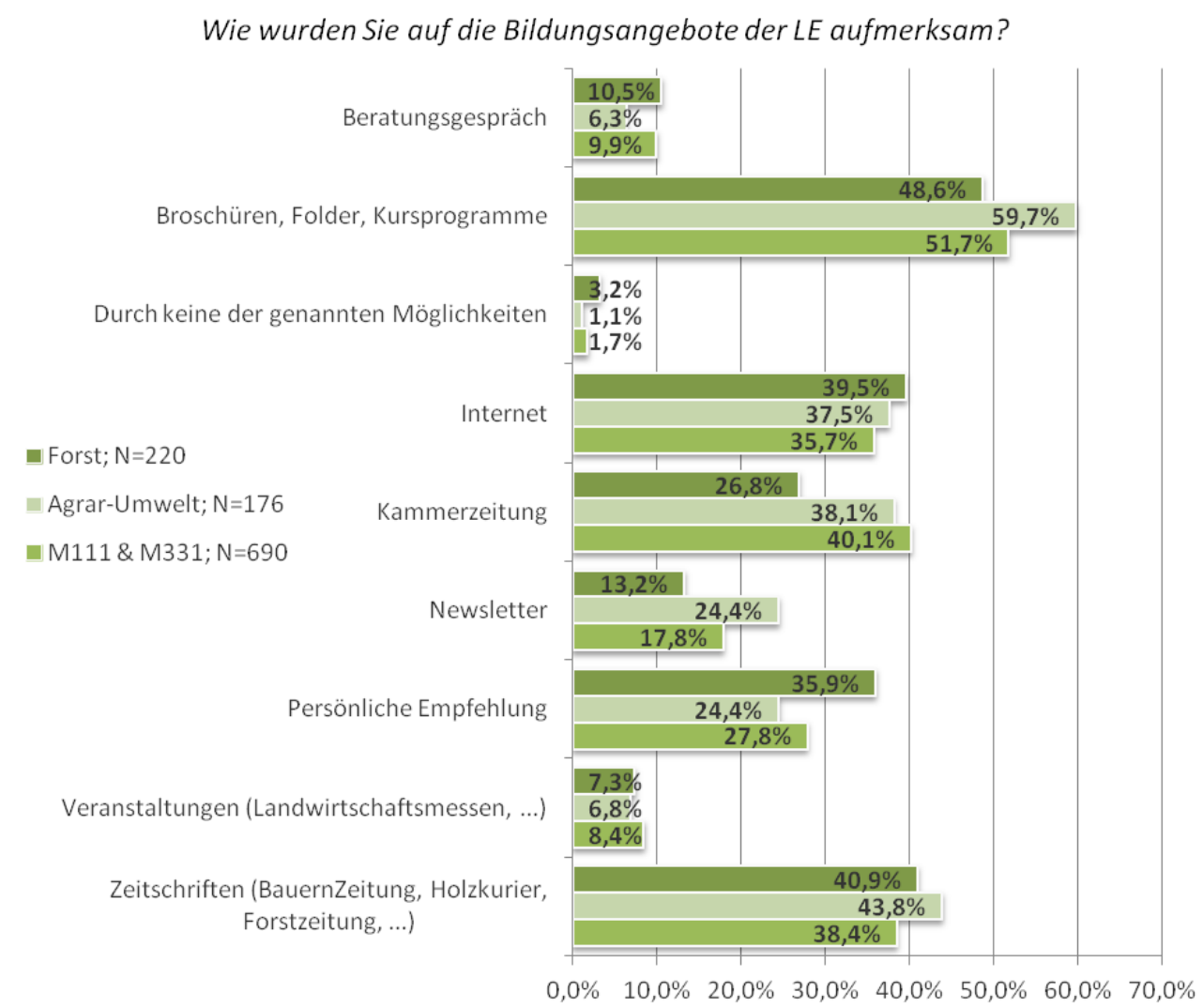

Die Bewerbung der Bildungsangebote wurde auch in den zur Verfügung gestellten Dokumenten beschrieben:

„Präsentation der FAST Ossiach bei der Austrofoma 2011: Die Forstfachmesse Austrofoma bietet der FAST die Möglichkeit ihr Bildungsangebot einem breiten forstlichen Publikum näher zu bringen. Auf einem eigenen Stand standen an den drei Besuchertagen Mitarbeiter der FAST für Bildungsberatung zur Verfügung, unterstützt durch Informationsposter und diverse Folder. Mehr als 20.000 Personen besuchten die Fachmesse im Forstbetrieb Stift Rein in der Stmk." (siehe Anhang III, Dok. [11], S. 4)

Weiters wurde hinsichtlich der Analyse des Bedarfs erhoben, welche Kriterien bei der Auswahl der Kurse und Veranstaltungen für die Land- bzw. ForstwirtInnen von Bedeutung sind. Dazu wurden die TeilnehmerInnen gebeten, die verschiedenen vorgegebenen Kriterien anhand der Kategorien "sehr wichtig" (100\%), „wichtig“ (50\%) und "unwichtig“ (0\%) zu bewerten.

Bei der Auswahl von Bildungsangeboten sind die Kriterien in den drei Gruppen sehr ähnlich gewichtet. Dabei sind "Thema", ,Erwarteter persönlicher Nutzen" und "Qualifikation und Kompetenz der Trainerin / des Trainers" die wichtigsten Faktoren bei der Kursselektion. „Bekanntheit und Image des Veranstalters" wird hingegen von den Teilnehmern aller betrachteten Bildungsmaßnahmen am unwichtigsten eingestuft.

In diesem Zusammenhang fiel im Zuge der Erstellung dieses Evaluierungsberichtes bei der Durchsicht der verschiedenen Bildungsangebots-Kataloge auf, dass bei den einzelnen Kursen bzw. Veranstaltungen die jeweiligen Kursleiter zwar namentlich angeführt werden, aber keine weiteren Informationen über deren beruflichen Werdegang bzw. deren Qualifikation gegeben werden. 
Besonders bemerkenswert ist, dass von allen 10 Auswahlkriterien die Kosten erst an 8. Stelle stehen. Nur "Bekanntheit und Image des Veranstalters" sowie „Empfehlungen von anderen" hat noch weniger Bedeutung.

Abbildung 54: Auswahlkriterien - Vergleich nach Bildungsmaßnahmen

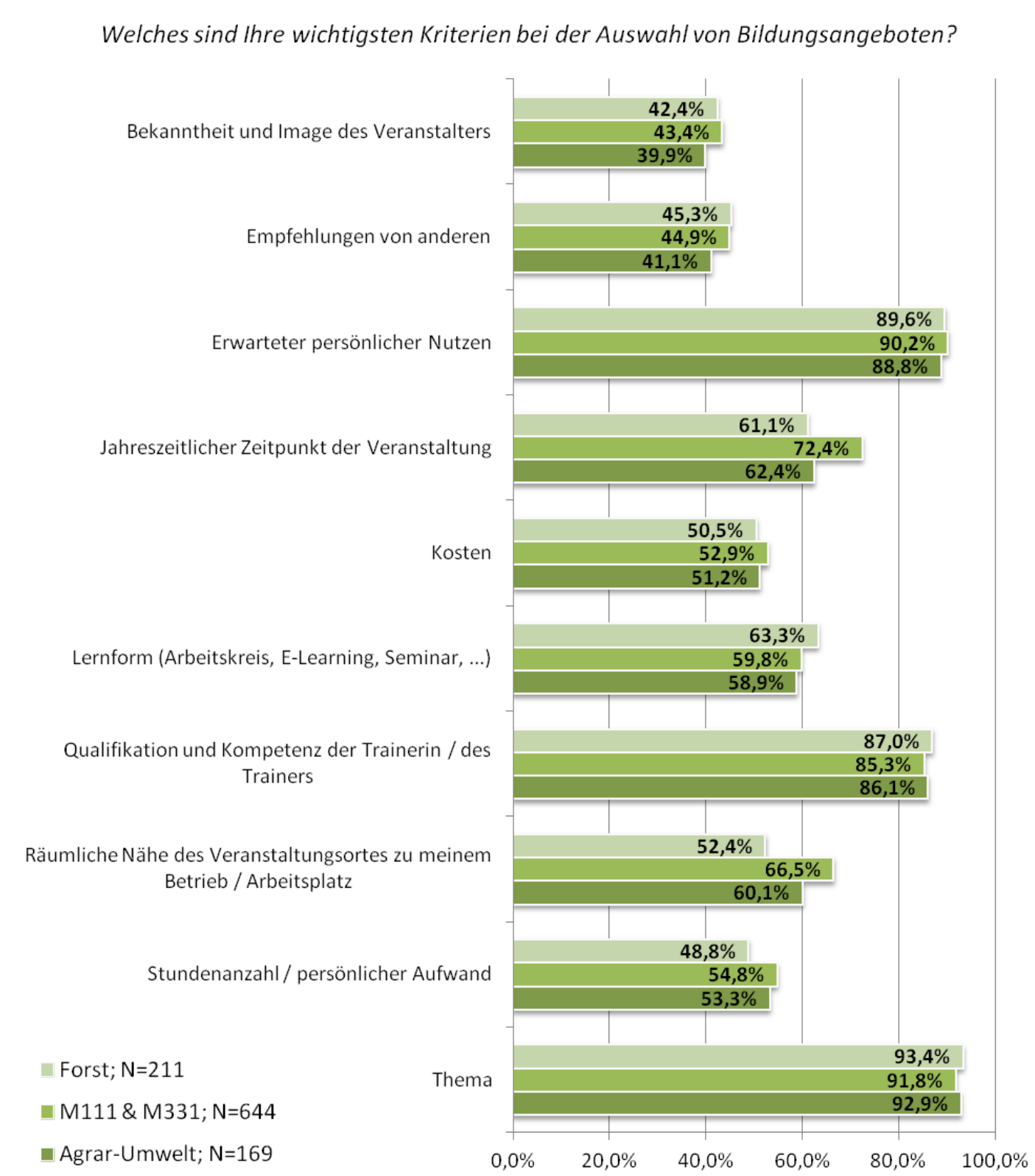

\section{Gender}

Um Unterschiede zwischen den Antworten von Frauen und Männern zu den oben angeführten Fragen und Darstellungen zu „Bedarf" festzustellen, werden im Folgenden vergleichende Grafiken präsentiert.

Sowohl Frauen als auch Männer wählten beim Thema "Kommunale und soziale Dienstleistungen" mehrheitlich die Kategorie "keine Angabe", die weiblichen Befragten bewerteten zudem auch "Erhaltung und Verbesserung des ländlichen Erbes" zum Großteil in dieser Kategorie. Gleichzeitig ist bei beiden aber auch die Anzahl derjenigen, die bzgl. dieses Themenfeldes die Kategorie "zu gering" wählten im Vergleich mit den anderen Themenfeldern sehr hoch bzw. bei den Frauen am 
höchsten. Männer wünschen sich vor allem im Bereich „Nachwachsende Rohstoffe und erneuerbare Energie" mehr Bildungsangebote.

\section{Abbildung 55a: Bewertung des Bildungsangebotes (absolut) - Gender}

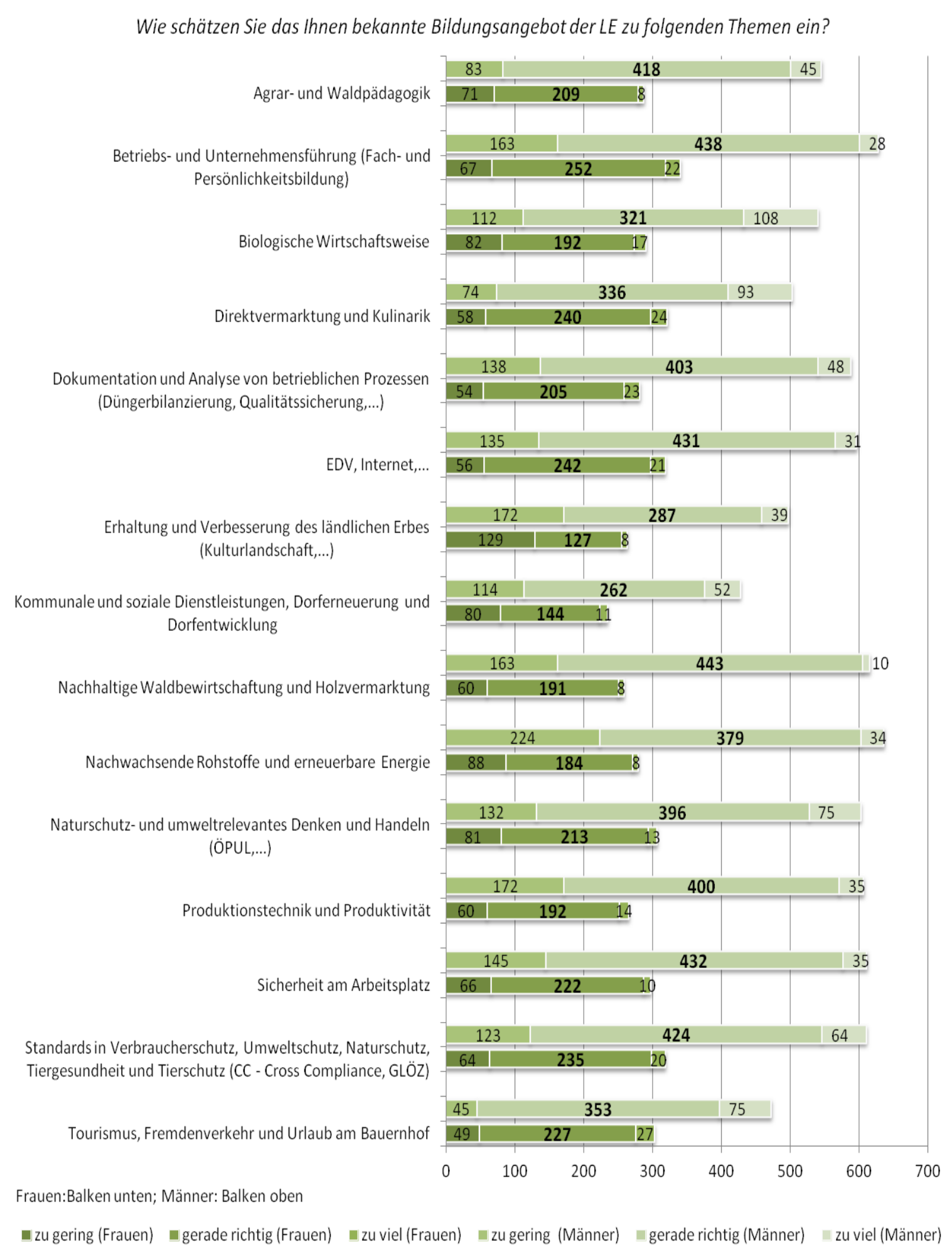


Abbildung 55b: Bewertung des Bildungsangebotes (in \%) - Gender

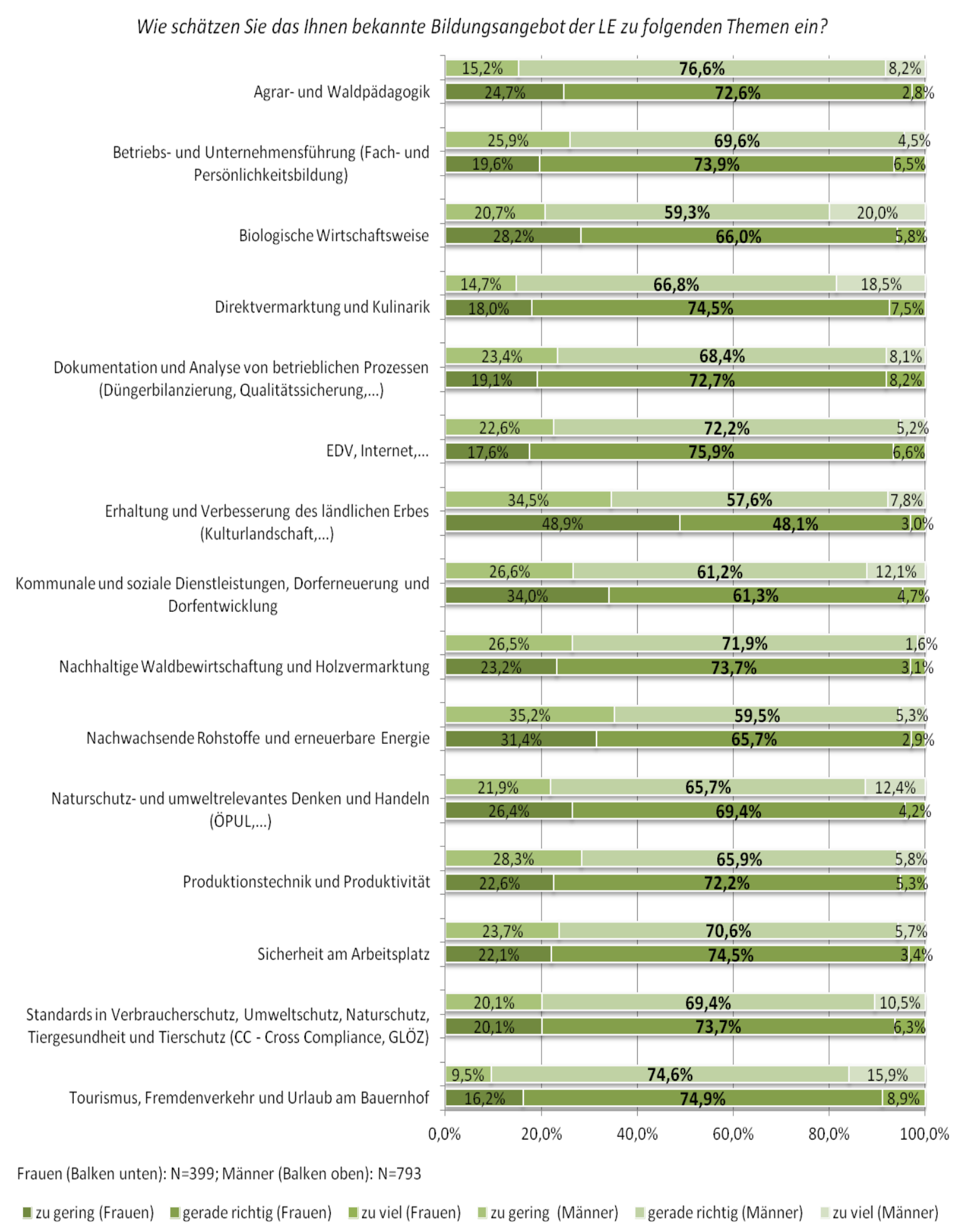


Deutliche Unterschiede zwischen Frauen und Männern bzgl. der Nachfrage an Kursen zu bestimmten Themen lassen sich anhand der Auswertung der Frage nach den besuchten Kursen erkennen. Die befragten Frauen nahmen vor allem an Kursen zu Betriebs- und Unternehmensführung, Direktvermarktung und Kulinarik sowie Standards in Verbraucherschutz etc. teil. Männer hingegen waren neben den Kursen zu Betriebs- und Unternehmensführung vor allem an Angeboten zur nachhaltigen Waldbewirtschaftung sowie zu Produktionstechnik interessiert. Am wenigsten nachgefragt wurden von Frauen die Kurse zu nachwachsenden Rohstoffen und erneuerbarer Energie, von den Männern Kurse zum Thema Kommunale und soziale Dienstleistungen.

Abbildung 56: Besuchte Kurse - Gender

Zu welchen der folgenden Themen haben Sie Bildungsangebote der LE besucht?

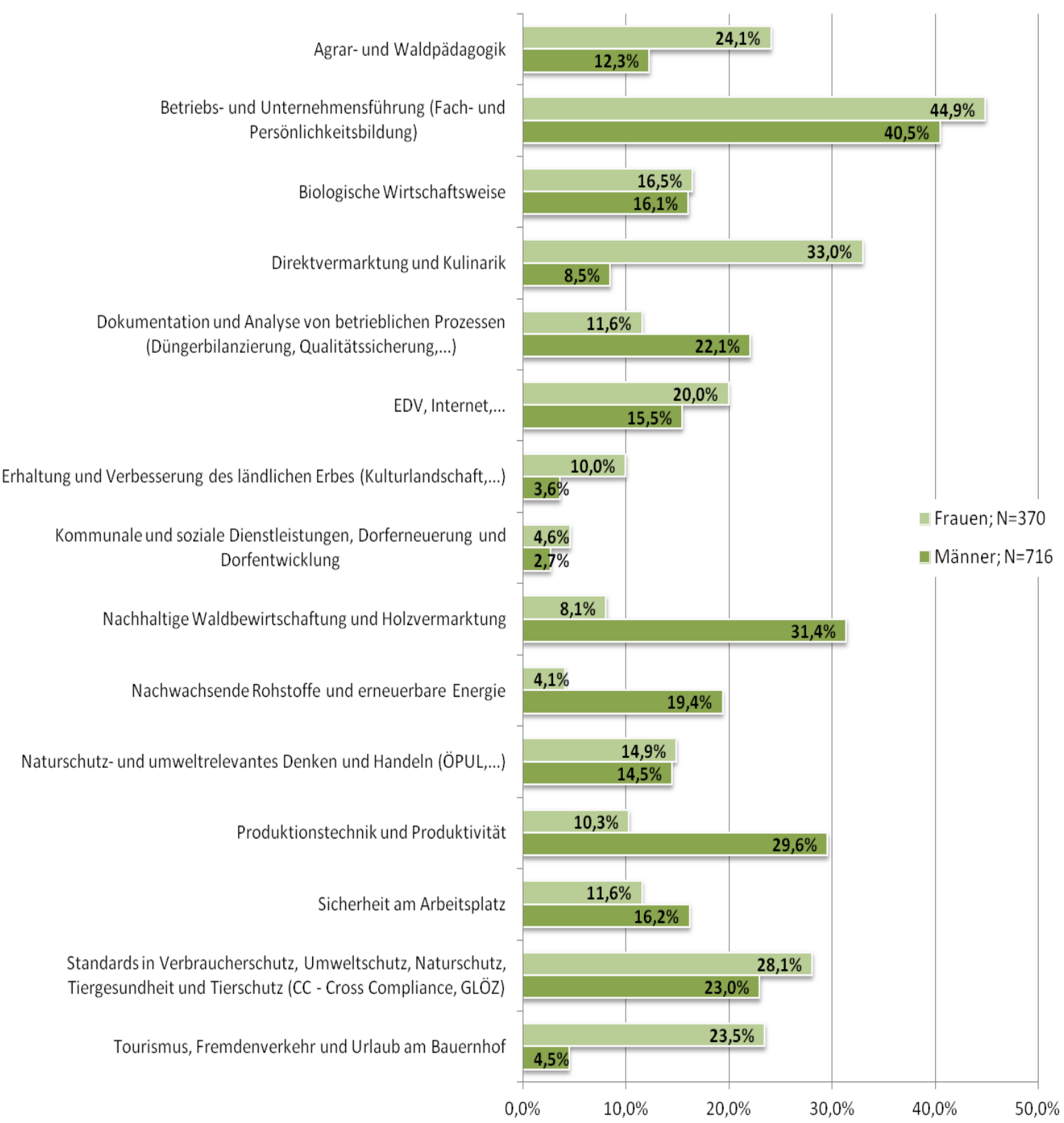


Broschüren und Folder waren sowohl für Frauen als auch für Männer bedeutsame Informationsquellen für Bildungsangebote der LE. 62,5\% der Frauen und 46,4\% der Männer gaben an, über dieses Medium von den Kursveranstaltungen erfahren zu haben. Am wenigsten informativ bzgl. der Bildungsangebote waren für Frauen Beratungsgespräche, und für Männer Veranstaltungen wie etwa Landwirtschaftsmessen.

Bei dieser Frage konnten in der Online-Umfrage maximal drei Antwortmöglichkeiten gewählt werden.

Abbildung 57: Informationsquellen zu den Bildungsangeboten - Gender

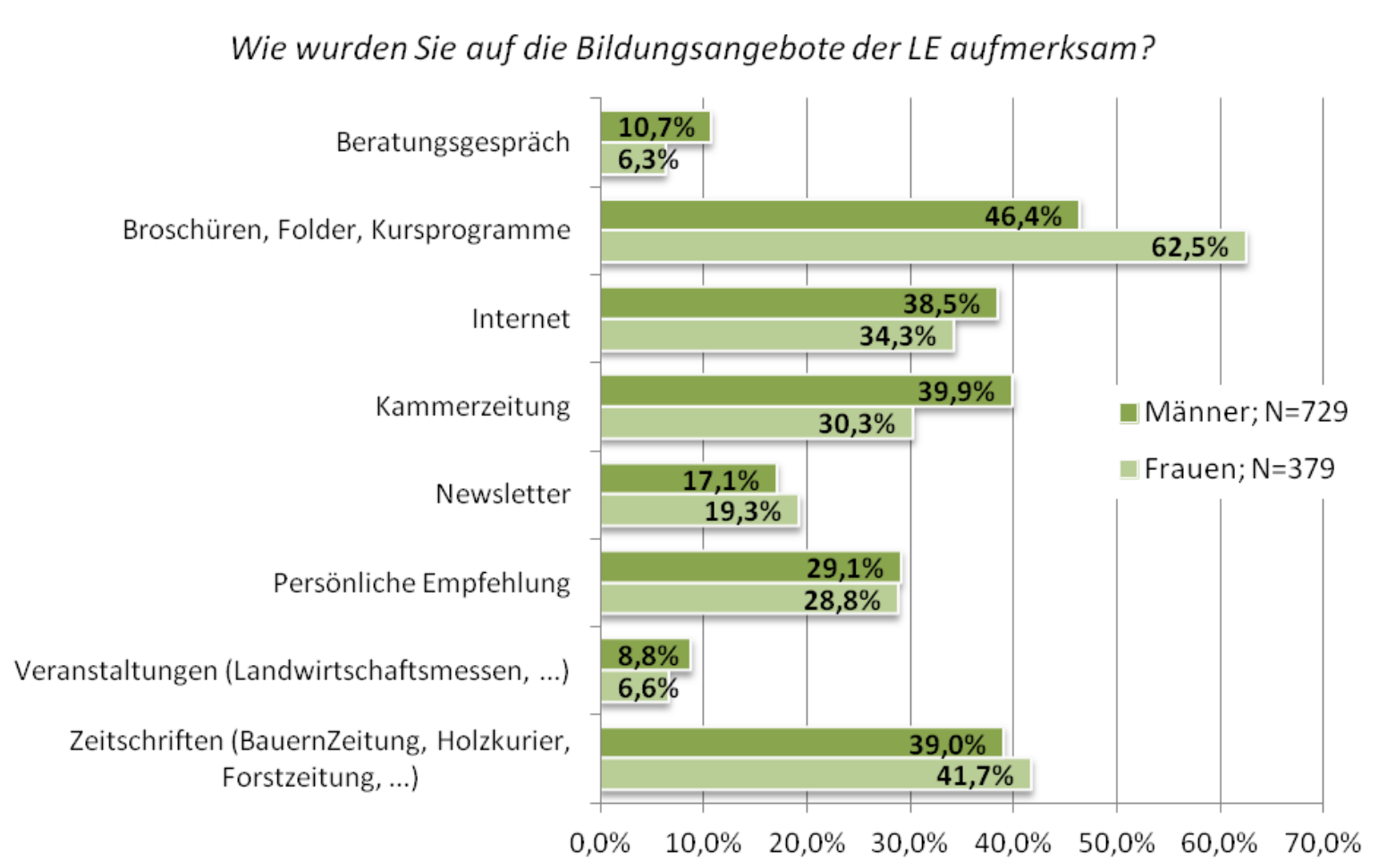

Bezüglich der Auswahl einzelner Kurse und Veranstaltungen bewerten Frauen und Männer die Bildungsangebote sehr ähnlich. Im Vergleich zu den befragten männlichen Land- bzw. Forstwirten spielt für Frauen die räumliche Nähe des Veranstaltungsortes zum eigenen Betrieb / Arbeitsplatz jedoch eine bedeutend größere Rolle. 
Abbildung 58: Auswahlkriterien - Vergleich nach Bildungsmaßnahmen

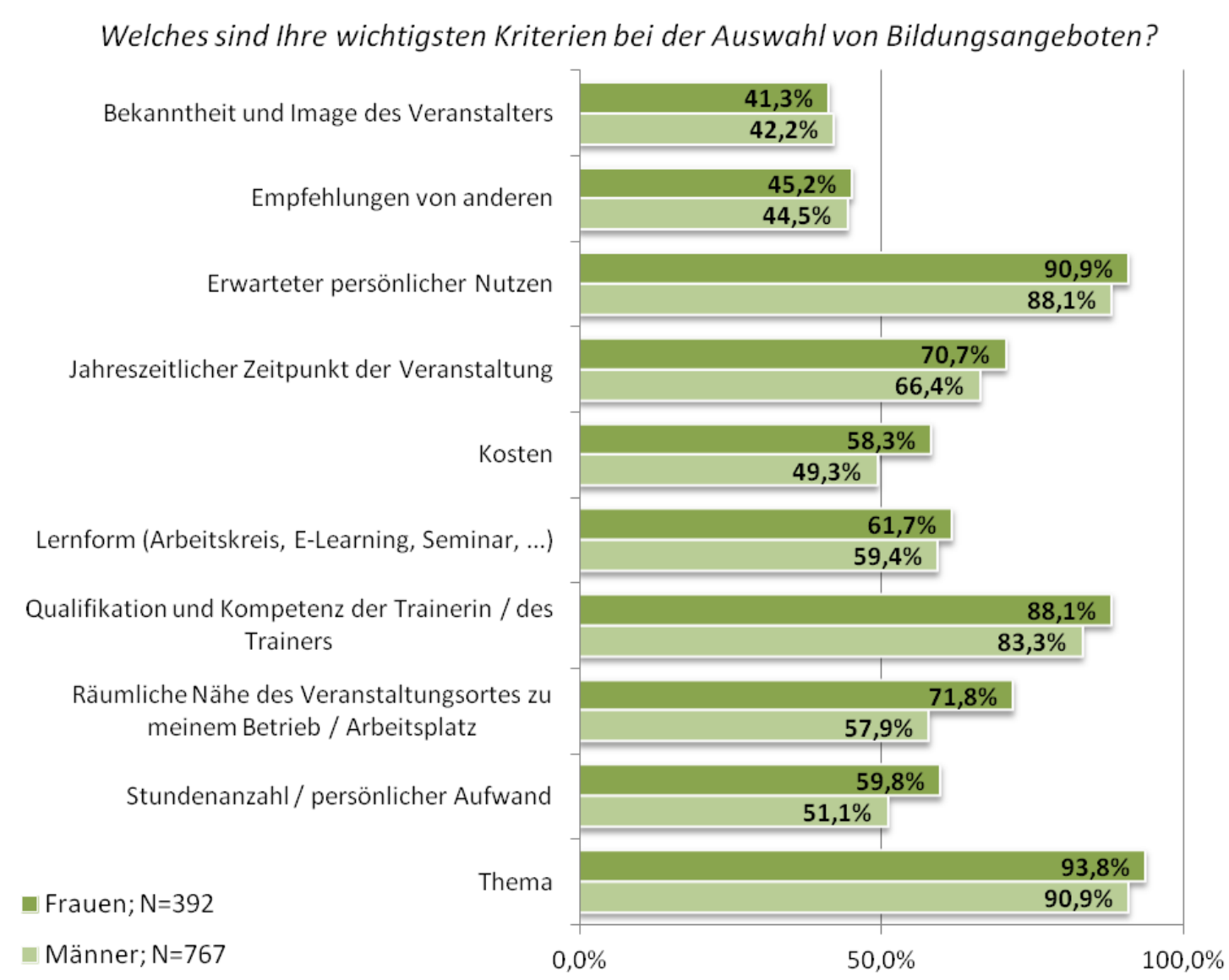

Seitens eines geförderten Veranstalters wurde zwar speziell zum Faktor "Kosten“ folgende Aussage in den Dialog Interviews getätigt:

"Landwirte können sich nicht geförderte Kurse gar nicht leisten, auch weil diese Ausgaben nicht steuer-reduzierend sind."

Diese Aussage wird indes aus Sicht der Land- bzw. ForstwirtInnen nicht bestätigt, denn sowohl für Frauen als auch für Männer rangieren die Kosten erst an vor-vorletzter Stelle.

\subsection{Verbesserung des Humanpotenzials}

Wie sehr die Bildungsangebote der LE zur Verbesserung des Humanpotenzials beigetragen haben wird in diesem Kapitel anhand der Auswertung der Online-Befragung bzgl. des Nutzens dargestellt. Diese zentrale Frage aus dem Fragebogen wird ergänzt durch eine vergleichende Auswertung zur Bewertung der in den Kursen zum Einsatz gekommenen Lernformen.

\section{Bildungsmaßnahmen M 111 \& M 331}

Im Vergleich zu den Antworten aus den Gruppen Agrar-Umwelt und Naturschutz und Forstbildungsmaßnahmen stuften Personen, die Kurse aus M 111 und M 331 besucht haben den Nutzen etwas weniger positiv ein - in dieser Gruppe wurde die Kategorie „Nützlich" häufiger gewählt, als in den beiden nachfolgend abgebildeten Gruppen. 
Abbildung 59: Empfundener Nutzen der Bildungsangebote - M 111 \& M 331

Wie nützlich fanden Sie die Bildungsangebote der LE für Ihre berufliche Weiterentwicklung insgesamt?

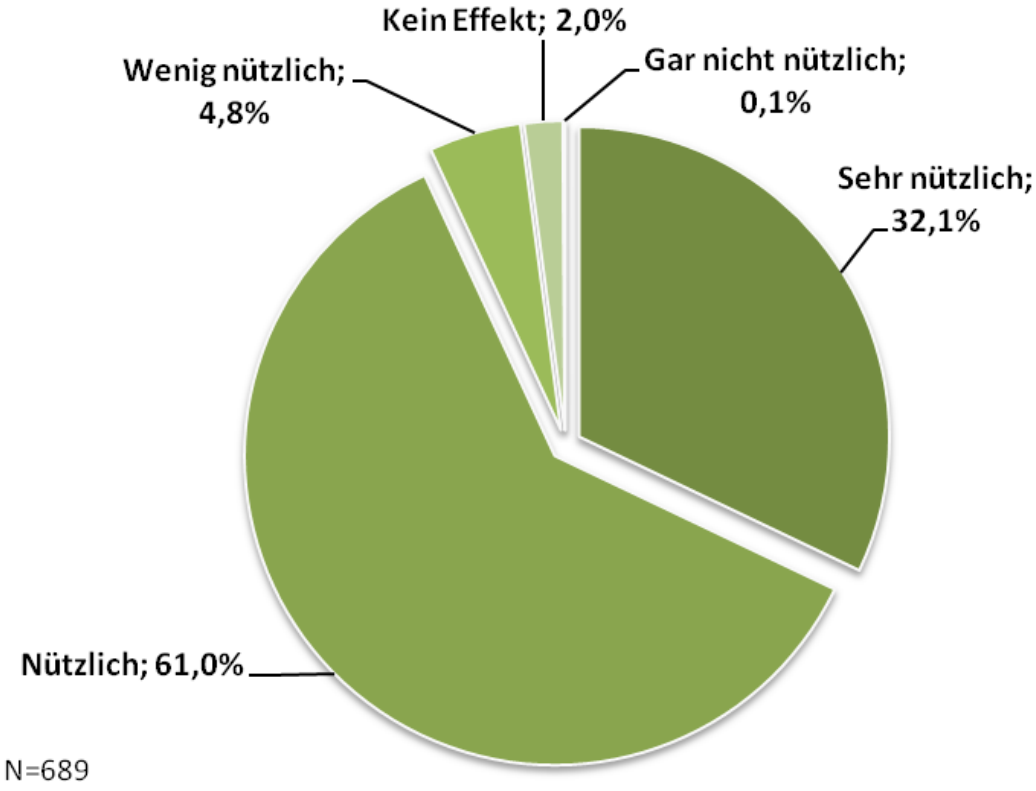

@ Sehr nützlich - Hat mich entscheidend weiter gebracht.

Nützlich - Ich habe neue / weiterführende Impulse für meine berufliche Tätigkeit erhalten.

Wenig nützlich - Geringe Verbesserung zu früher feststellbar.

- Kein Effekt - Es ist für mich kein Unterschied zu früher feststellbar.

Gar nicht nützlich - Hat mich auf einen falschen beruflichen Weg gebracht.

\section{Agrar-Umwelt und Naturschutz}

Die Kategorie „gar nicht nützlich“ wurde in dieser Gruppe nicht ausgewählt.

Abbildung 60: Empfundener Nutzen der Bildungsangebote - Agrar-Umwelt und Naturschutz

Wie nützlich fanden Sie die Bildungsangebote der LE für Ihre berufliche Weiterentwicklung insgesamt?

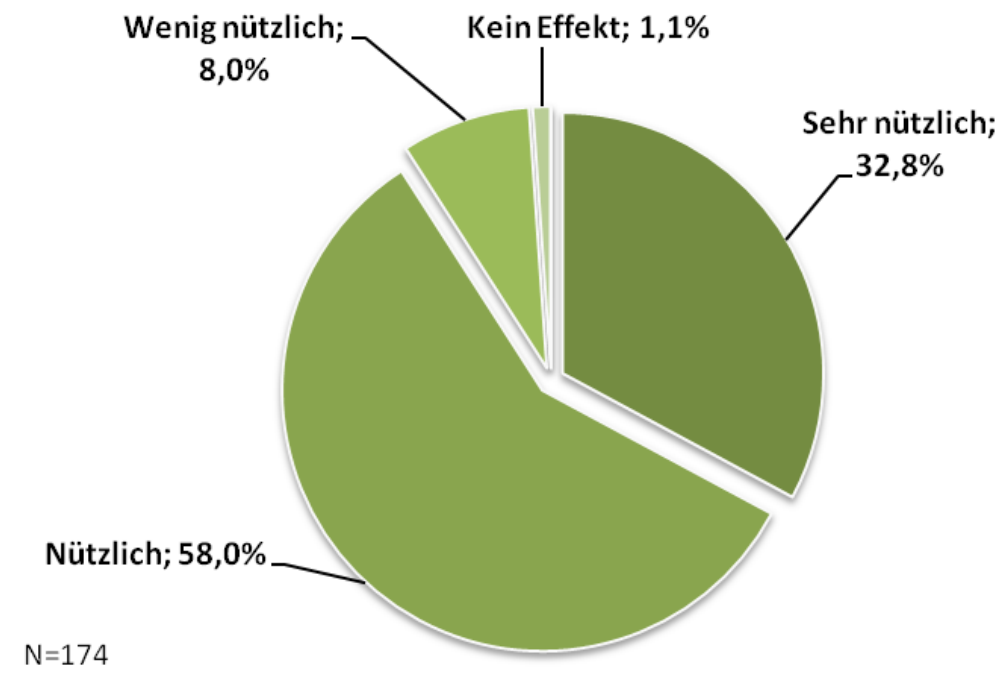

- Sehr nützlich - Hat mich entscheidend weiter gebracht.

Nützlich - Ich habe neue / weiterführende Impulse für meine berufliche Tätigkeit erhalten.

- Wenig nützlich - Geringe Verbesserung zu früher feststellbar.

Kein Effekt - Es ist für mich kein Unterschied zu früher feststellbar.

- Gar nicht nützlich - Hat mich auf einen falschen beruflichen Weg gebracht. 


\section{Forstbildungsmaßnahmen}

In der Gruppe der Personen, die Bildungsmaßnahmen aus dem forstlichen Bereich besuchten ist der Anteil derer, die diese Bildungsangebote als sehr nützlich empfinden deutlich größer, als bei den zuvor beschriebenen Gruppen. Die Kategorie "gar nicht nützlich“ wurde in dieser Gruppe nicht ausgewählt.

Abbildung 61: Empfundener Nutzen der Bildungsangebote - Forstbildungsmaßnahmen

Wie nützlich fanden Sie die Bildungsangebote der LE für Ihre berufliche Weiterentwicklung insgesamt?
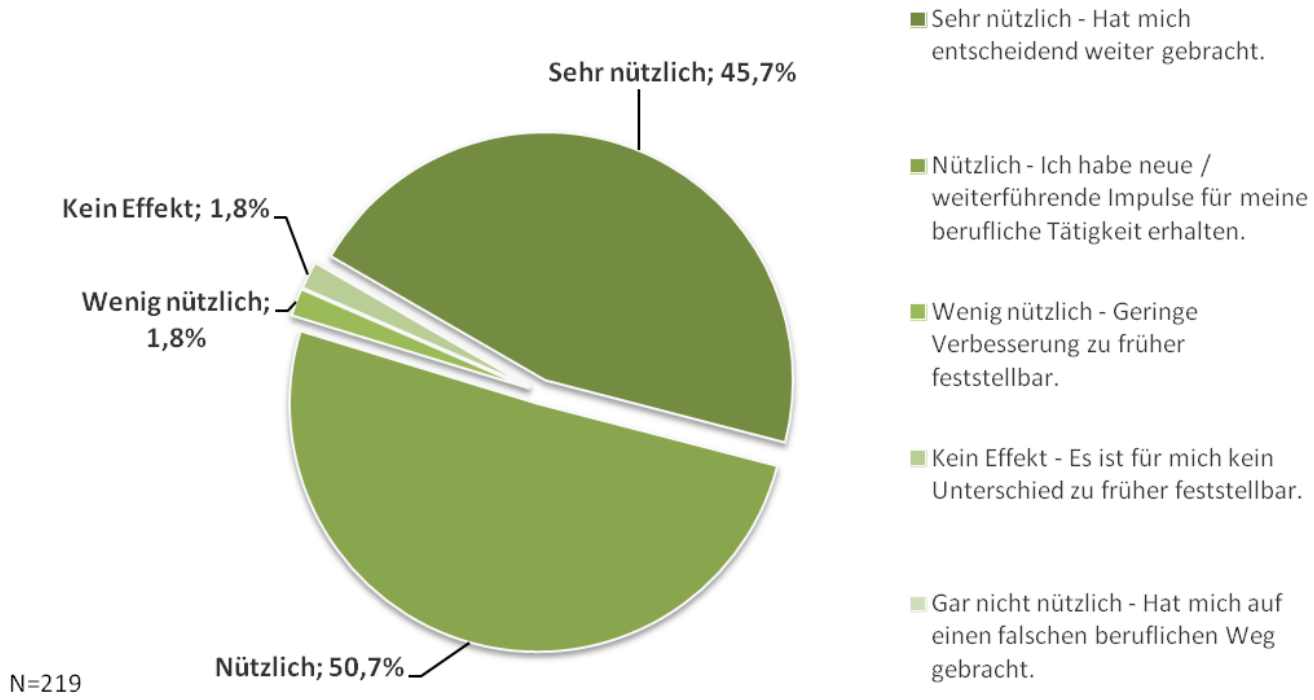

Der Nutzen einer Bildungsveranstaltung hängt neben dem jeweiligen Thema auch mit den zum Einsatz kommenden Lernformen bzw. methodischen Ansätzen und gewählten Kursformen zusammen. Besonders beliebt sind bei allen drei Vergleichsgruppen die Lernformen "Seminare und Kurse", „Betriebsbesuche / Exkursionen" und „Erfahrungsaustausch und Arbeitskreise". 
Abbildung 62: Lernformen - Vergleich nach Bildungsmaßnahmen

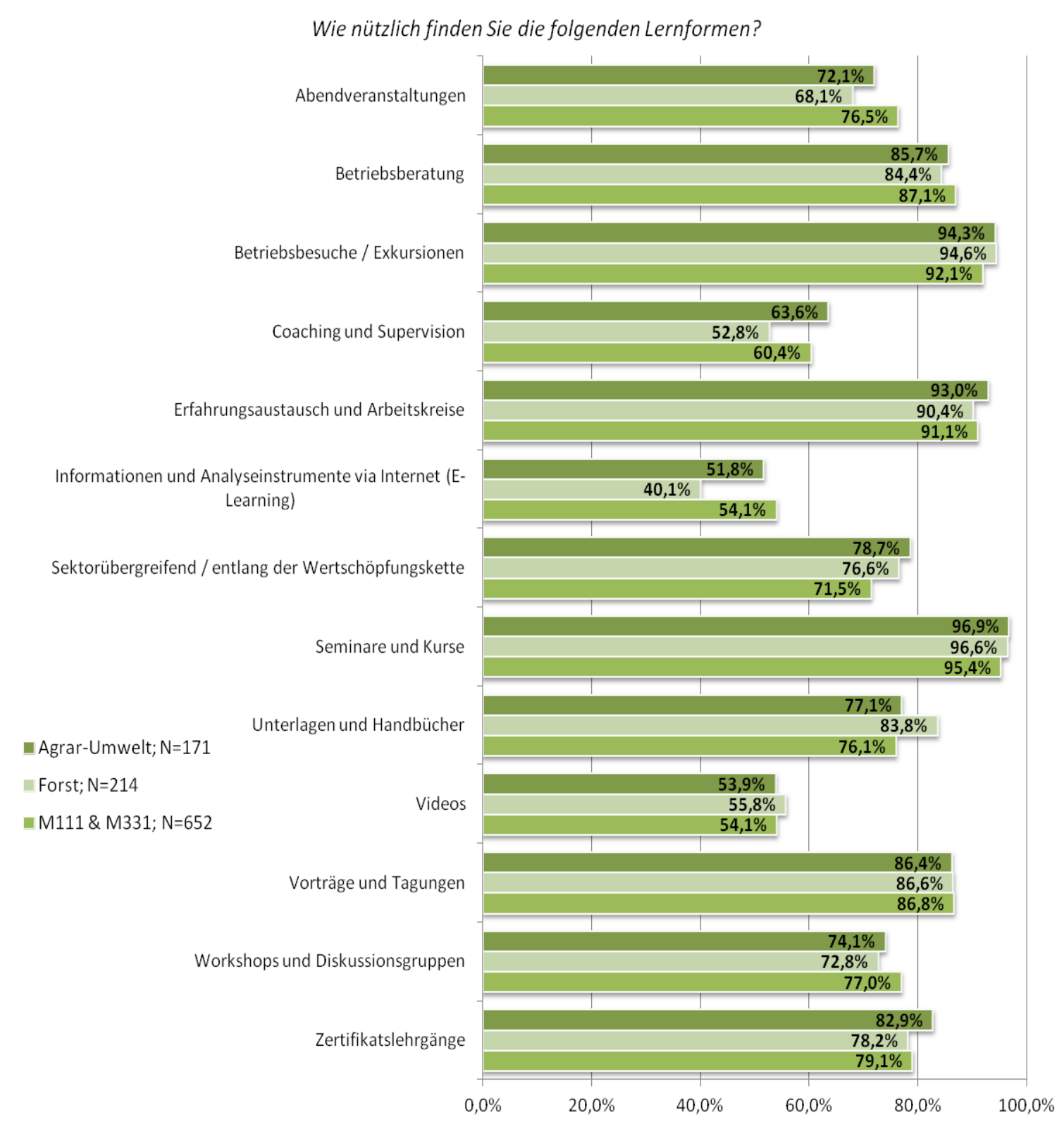

In den Dialog Interviews wurde folgendes zu den Lernformen angemerkt:

„Der Trend geht in Richtung Zertifikatslehrgänge und damit zu längeren Ausbildungen."

"Weiterbildungsseminare / -Kurse via Partner LFI werden schlecht angenommen, Know-HowTransfer direkt vor Ort hingegen, betriebsindividuell, wird sehr gut angenommen." 


\section{Gender}

In ihrer Empfindung bzw. Einschätzung zum Nutzen der besuchten Bildungsveranstaltungen liegen Frauen und Männer sehr eng beisammen. 93, 9\% der Männer und 92,2 \% der Frauen wählten bei der unten dargestellten Frage die Kategorien „Sehr nützlich“ und „Nützlich“.

Abbildung 63: Empfundener Nutzen der Bildungsangebote - Gender

Wie nützlich fanden Sie die Bildungsangebote der LE für Ihre berufliche Weiterentwicklung insgesamt?

Sehr nützlich - Hat mich entscheidend weiter gebracht.

Nützlich - Ich habe neue / weiterführende Impulse für meine berufliche Tätigkeit erhalten.

Wenig nützlich - Geringe Verbesserung zu früher feststellbar.
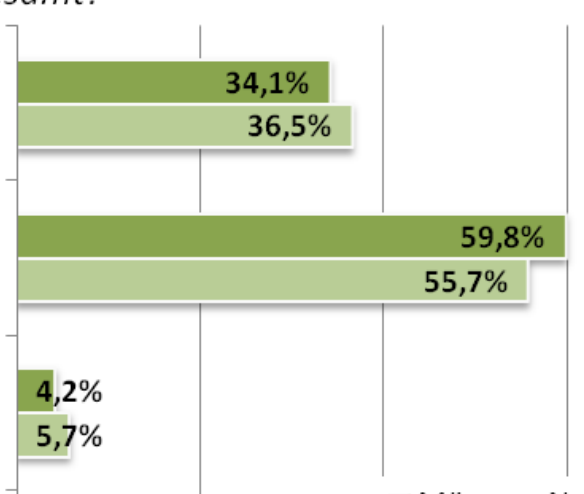

Kein Effekt - Es ist für mich kein Unterschied zu früher feststellbar.

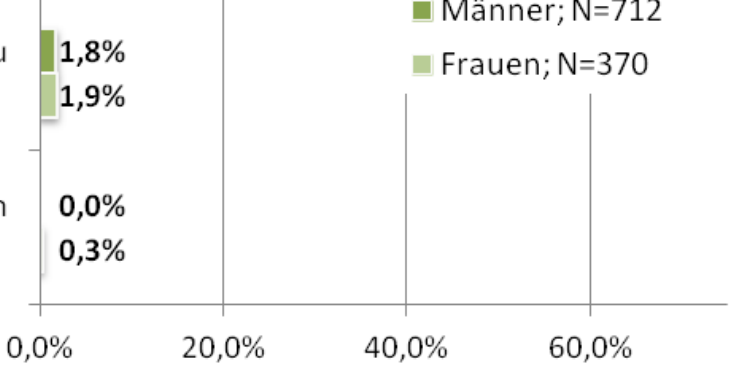

Wie oben bei der vergleichenden Betrachtung der drei Bildungsmaßnahmen-Gruppen zu den Lernformen dargestellt, sind "Seminare und Kurse", "Betriebsbesuche / Exkursionen" und "Erfahrungsaustausch und Arbeitskreise" die drei beliebtesten Lernformen. Diese Reihung verändert sich auch nicht bei der gendermäßigen Betrachtung der Antworten. Frauen haben bei fast allen Lernformen häufiger die Aussage „sehr nützlich" getroffen, als die Männer, besonders bei „Coaching und Supervision" und "Zertifikatslehrgänge" haben Frauen eine deutlich positivere Meinung (siehe Abbildung 64). 
Abbildung 64: Lernformen - Gender

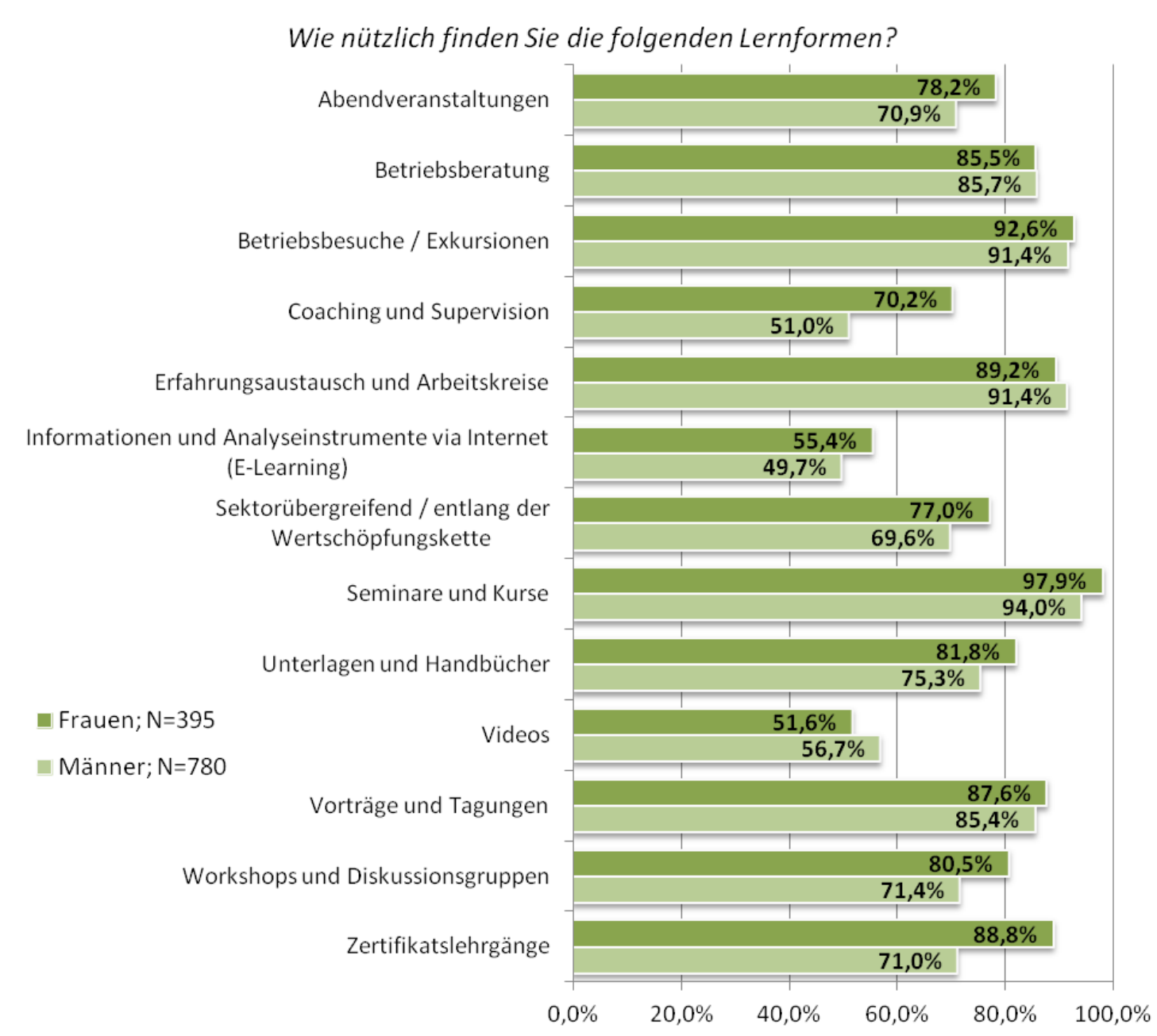

\subsection{Diversifizierung in nicht-agrarische Bereiche}

Bei der Frage nach einer zusätzlichen Tätigkeit in nicht-agrarischen Bereichen wurden den Teilnehmern mehrere Diversifizierungsbereiche vorgeschlagen. Die vorgegebenen Antwortmöglichkeiten beziehen sich auf mögliche, im Programm der LE festgelegte Bereiche.

In den Dialog Interviews wurde zum Thema Diversifikation in nicht-agrarische Bereiche folgende Aussage getätigt:

"Vorher gab es ca. 70 Produzenten, jetzt sind es 214. Daneben sind ca. 114 neue Produkte entstanden, sowie neue Naturparkläden. Diese stellen einen echten Zusatzverdienst für Naturpark-Bauern dar."

\section{Bildungsmaßnahmen M 111 \& M 331}

Die Frage erlaubte eine Auswahl von maximal zwei Bereichen. Fast die Hälfte der Land- bzw. ForstwirtInnen, die Bildungsveranstaltungen aus $M 111$ und M 331 besucht haben, sind jedoch in keinem der angeführten nicht-agrarischen Bereiche tätig. 
Abbildung 65: Nicht-agrarische Bereiche - M 111 \& M 331

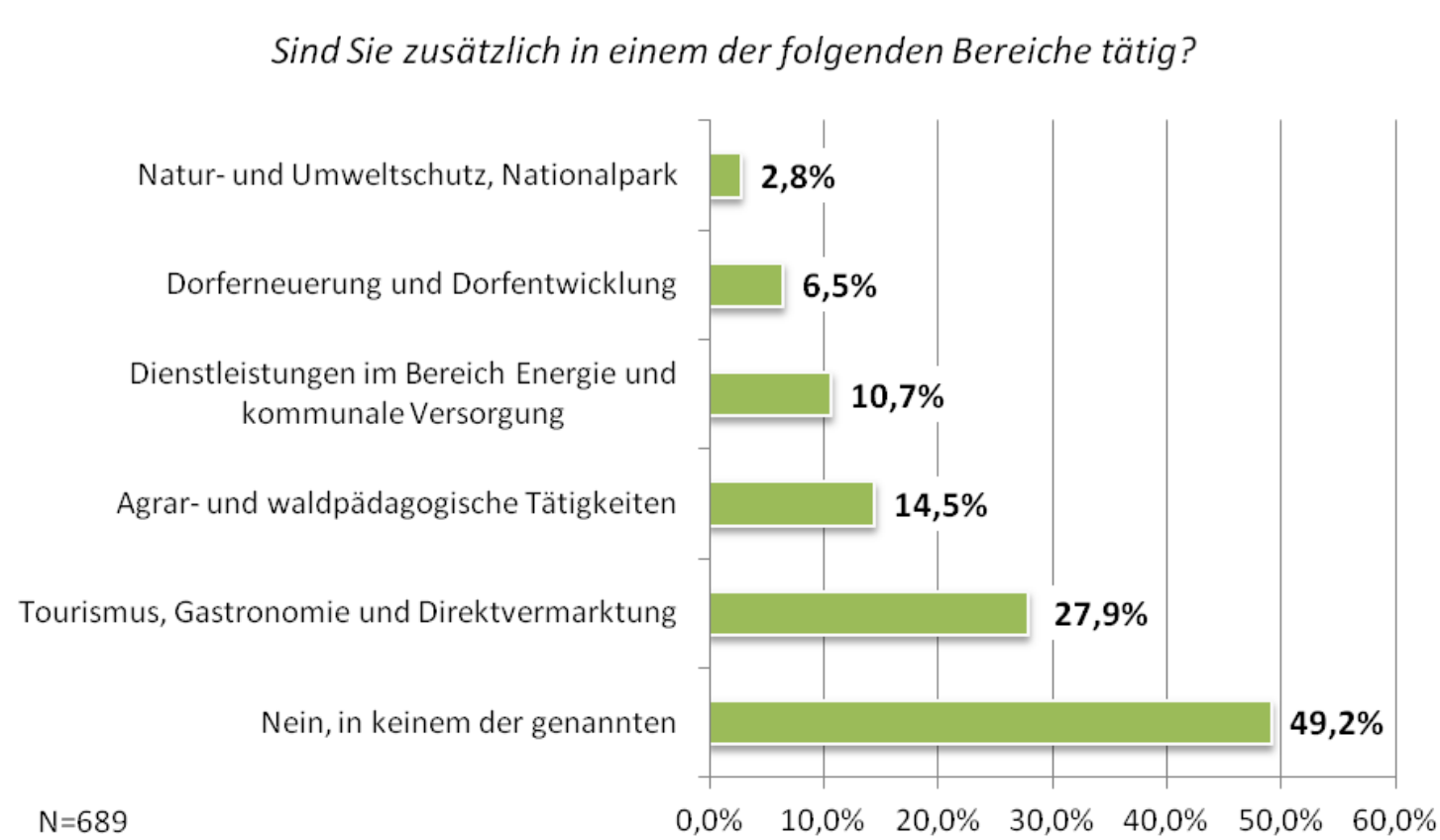

\section{Agrar-Umwelt und Naturschutz}

Jene Personen, die Kurse zu Agrar-Umwelt und Naturschutz besuchten, sind neben ihrer landbzw. forstwirtschaftlichen Tätigkeit hauptsächlich noch in Tourismus, Gastronomie und Direktvermarktung tätig, oder in agrar- und waldpädagogischen Aktivitäten engagiert. Diese Personen sind deutlich diversifizierter tätig als jene, welche Bildungsveranstaltungen aus M 111 und M 331 oder welche Kurse aus dem Forstbereich besucht haben.

Abbildung 66: Nicht-agrarische Bereiche - Agrar-Umwelt und Naturschutz

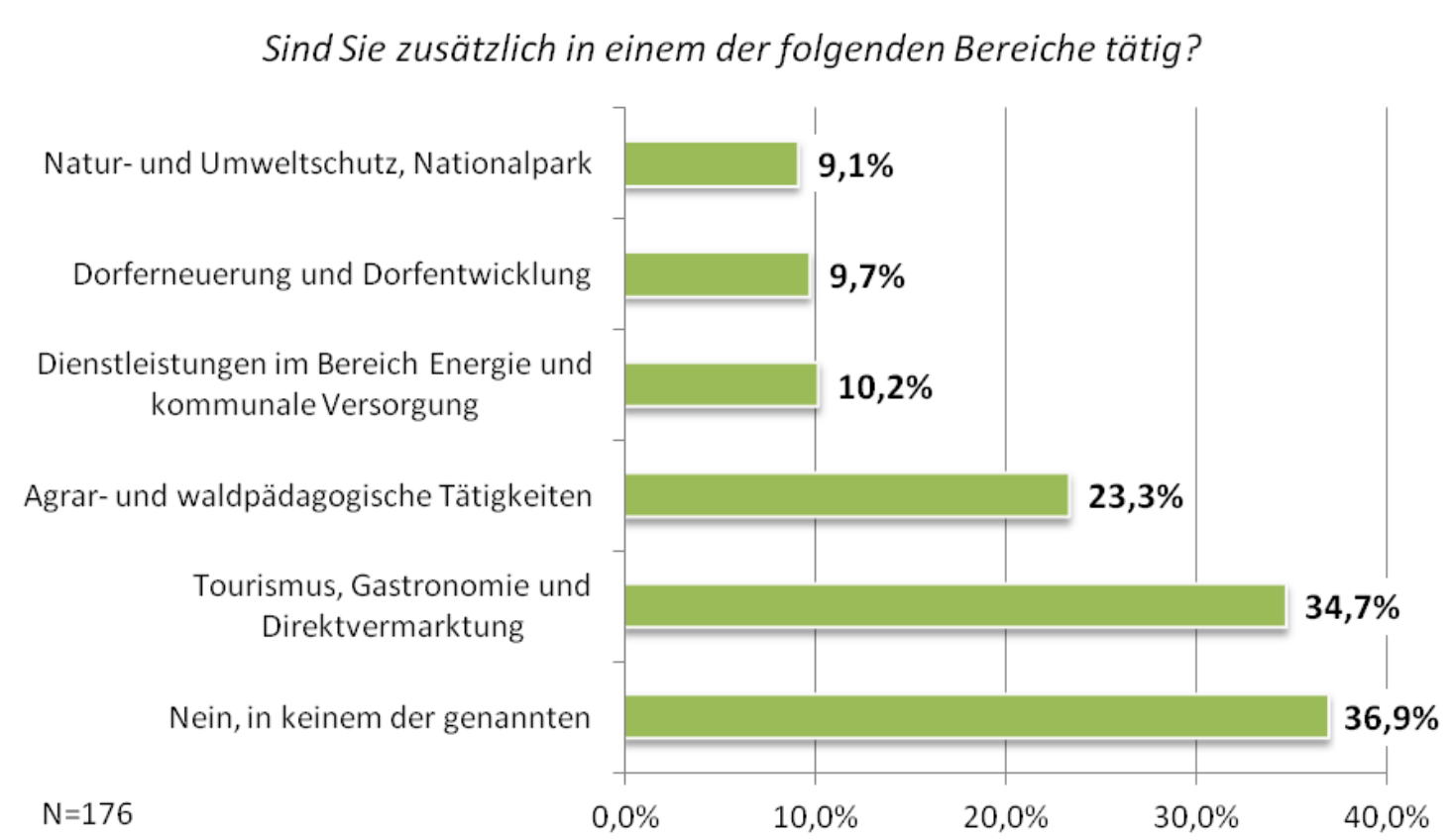




\section{Forstbildungsmaßnahmen}

Mehr als die Hälfte der Personen aus der Land- bzw. Forstwirtschaft, die Kurse aus dem Forstbereich besuchten, sind nicht zusätzlich in nicht-agrarischen Bereichen tätig.

Abbildung 67: Nicht-agrarische Bereiche - Forstbildungsmaßnahmen

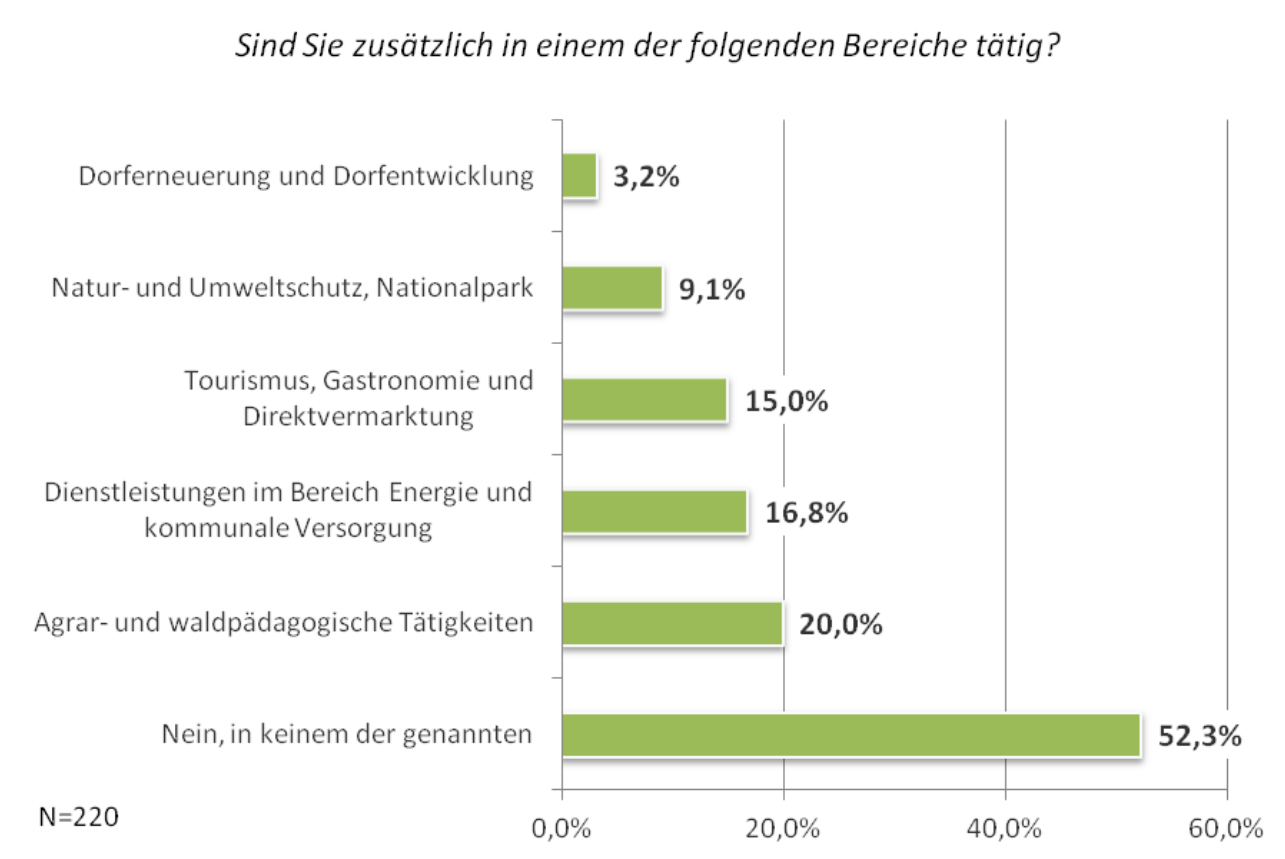

Land-bzw. ForstwirtInnen, die keine Bildungsangebote besucht haben

Auch Land- bzw. ForstwirtInnen, die keine Kurse der LE absolvierten, diversifizieren in nichtagrarische Bereiche. Im Vergleich mit Land- bzw. ForstwirtInnen, die an LEBildungsveranstaltungen teilgenommen haben, fällt jedoch auf, dass letztere deutlich stärker diversifizieren.

Abbildung 68: Nicht-agrarische Bereiche - Forstbildungsmaßnahmen

Sind Sie zusätzlich in einem der folgenden Bereiche tätig?

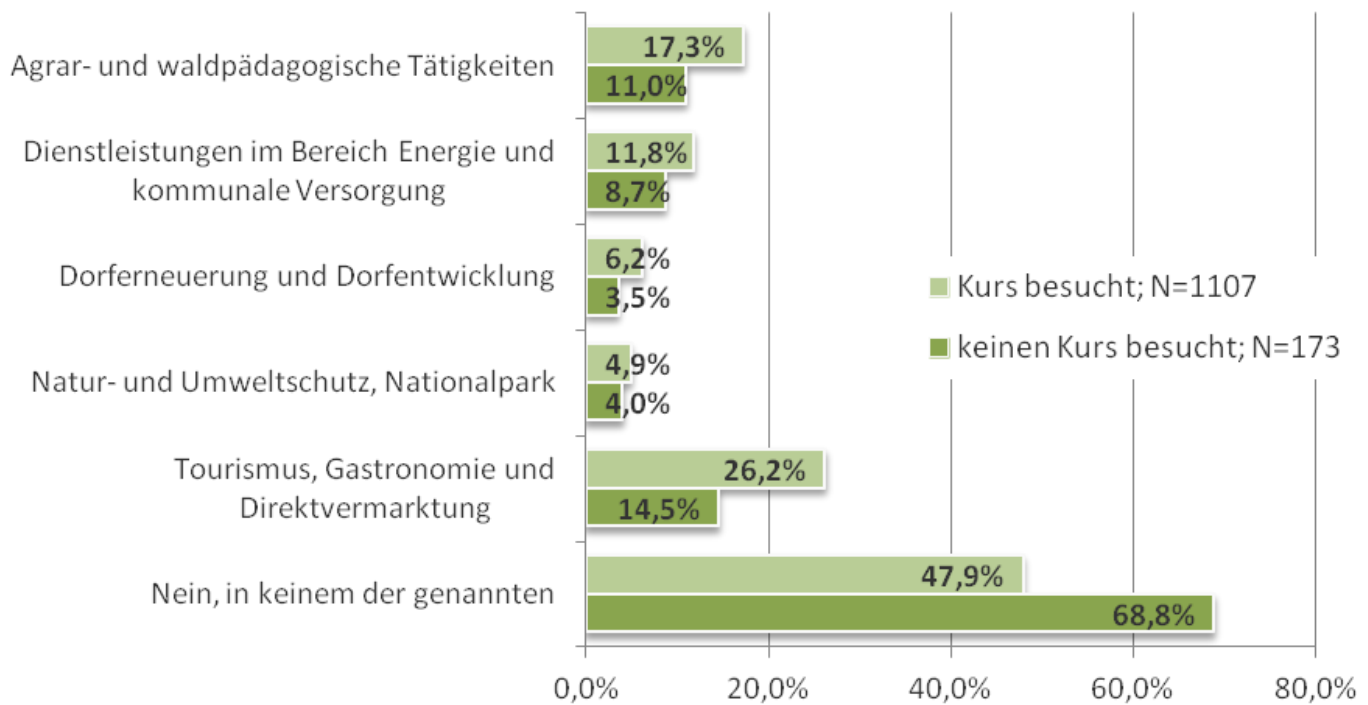




\section{Gender}

Die befragten Frauen sind im Vergleich zu den Männern mehr in nicht-agrarische Tätigkeiten involviert - dabei vor allem in Tourismus, Gastronomie und Direktvermarktung sowie Agrar- und Waldpädagogik. Die Kategorie "Dienstleistungen im Bereich Energie und kommunale Versorgung" wurde jedoch deutlich häufiger von Männern, als von Frauen ausgewählt.

Abbildung 69: Nicht-agrarische Bereiche - Gender

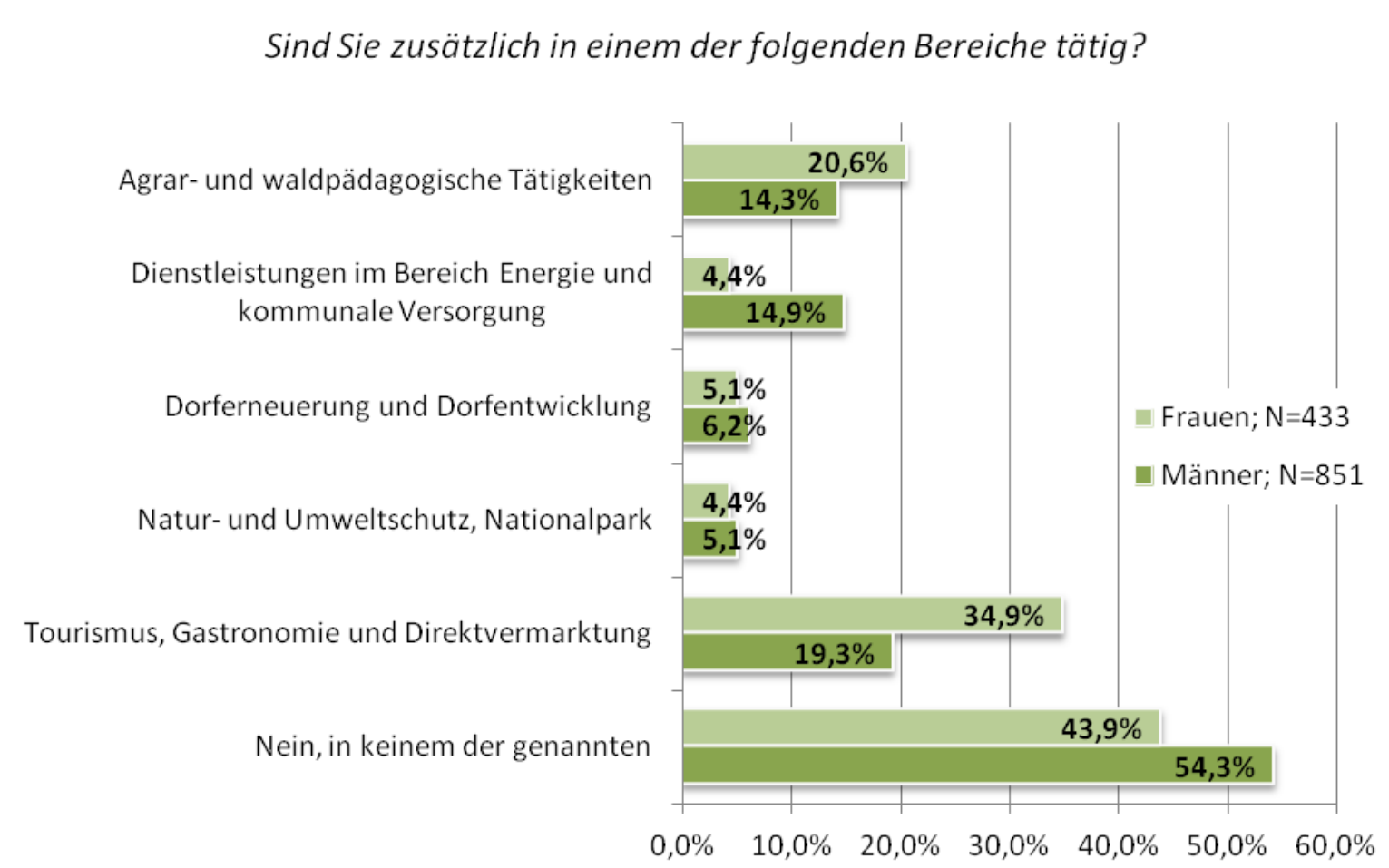

\subsection{Einsatz des erlangten Wissens}

Zum Einsatz des in den Bildungsangeboten erworbenen Wissens lassen sich einerseits aus den Antworten zu den in den Kapiteln 4.1-4.5 dargestellten Fragen aus dem Fragebogen Rückschlüsse ziehen, andererseits gibt auch der zuvor abgebildete Vergleich zwischen Land- bzw. ForstwirtInnen, die Kurse besucht haben und jenen, die keine Kurse der LE besuchten, klare Hinweise (siehe Abbildung 68).

Abbildung 70 vergleicht nun die, von diesen beiden Gruppen wahrgenommene Veränderung im Betrieb bzw. im ländlichen Raum im Detail. Wie schon in der zusammenfassenden Graphik in Abbildung 25 (S. 33) ersichtlich, wurden in der Gruppe der Land- bzw. ForstwirtInnen, die Kurse besucht haben, die Veränderungen deutlich häufiger mit "mehr" bewertet. Dies deutet ebenfalls auf einen wechselseitigen Zusammenhang zwischen dem, durch Bildungsangebote der LE erworbenen neuen Wissen und den wahrgenommenen Veränderungen hin. 
Abbildung 70: Veränderung seit 2007 im Detail - Vergleich nach Teilnahme

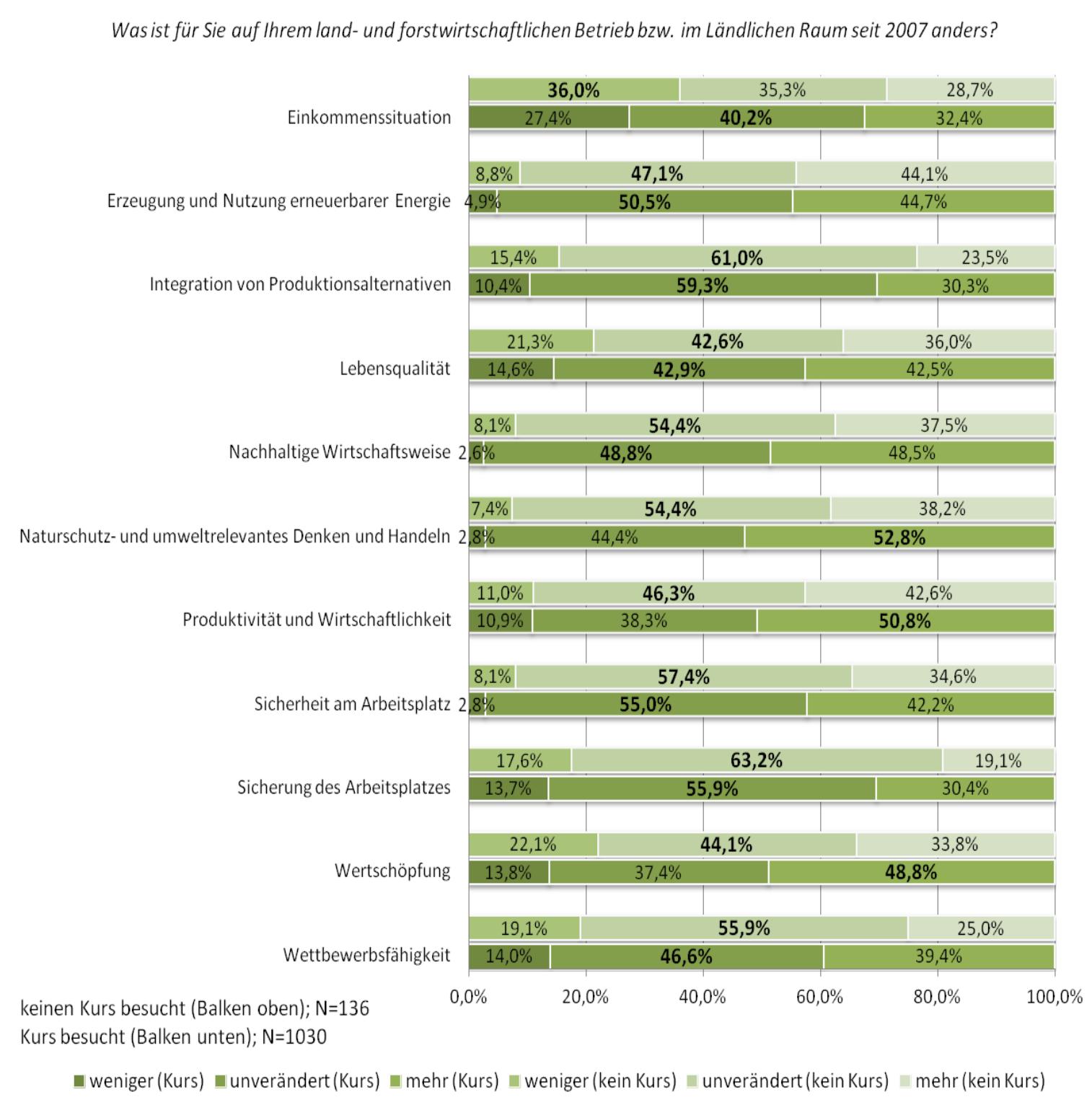

In den Dialog Interviews wurde auf das Thema „Einsatz des erlangten Wissens" folgendermaßen eingegangen:

„Man bekommt Mut, Neues umzusetzen und die Komfortzone zu verlassen (z.B. Umstrukturierung)."

„Wichtig für die Existenzssicherung der LW / FW"

"Wichtig für Zukunft: Persönlichkeitsbildung (mit sich selbst befassen)“"

„Jungzüchter setzen im Betrieb ihrer Eltern moderne Konzepte um.“

„ICh konnte nach dem Seminar über Kraftfutter die Milchproduktion steigern - um 1.200I pro Kuh/ Jahr." 
„Das Euter-Gesundheits-Seminar brachte Erkenntnisse bzgl. Hygiene und war hilfreich bei der Bekämpfung von Krankheitserregern."

„Der Nutzen ist für mich einerseits die Bildung - es gibt immer neue Erkenntnisse in der Landwirtschaft - andererseits der Erfahrungsaustausch mit anderen Landwirten, aber auch die Dokumentation und Analyse der Betriebsdaten."

„Man geht viel aufmerksamer durch den Wald und sieht und erkennt Krankheiten schon früher."

\subsection{Verbesserung der Lebensqualität}

Die nachstehend angeführten Abbildungen zeigen Detailauswertungen der in Kapitel 4.1 betrachteten Frage aus der Online-Befragung "Was ist für Sie auf Ihrem land- und forstwirtschaftlichen Betrieb bzw. im Ländlichen Raum seit 2007 anders?". Bei einer vergleichenden Betrachtung der unterschiedlichen Bildungsmaßnahmen werden Unterschiede deutlich. Besonders jene Gruppe, die an Bildungsangeboten zu Agrar-Umwelt und Naturschutz teilgenommen hat, sieht zum Thema „Lebensqualität" seit 2007 Großteils eine Veränderung ins Positive.

In den Dialog-Interviews gingen die Interviewpartner ebenfalls auf dieses Thema ein:

„Imagegewinn für Landwirtschaft. Das Tier bekommt einen anderen Stellenwert und ist nicht mehr einfach nur Fleischlieferant."

„Bäuerinnen können sich in ihrer pädagogischen oder therapeutischen Praxis am Bauernhof verwirklichen."

\section{Bildungsmaßnahmen M 111 \& M 331}

58,5\% der Teilnehmer, die dieser Gruppe zugeordnet werden können, sehen keine Veränderung oder Verschlechterung bzgl. der Lebensqualität.

Abbildung 71: Lebensqualität - M 111 \& M 331

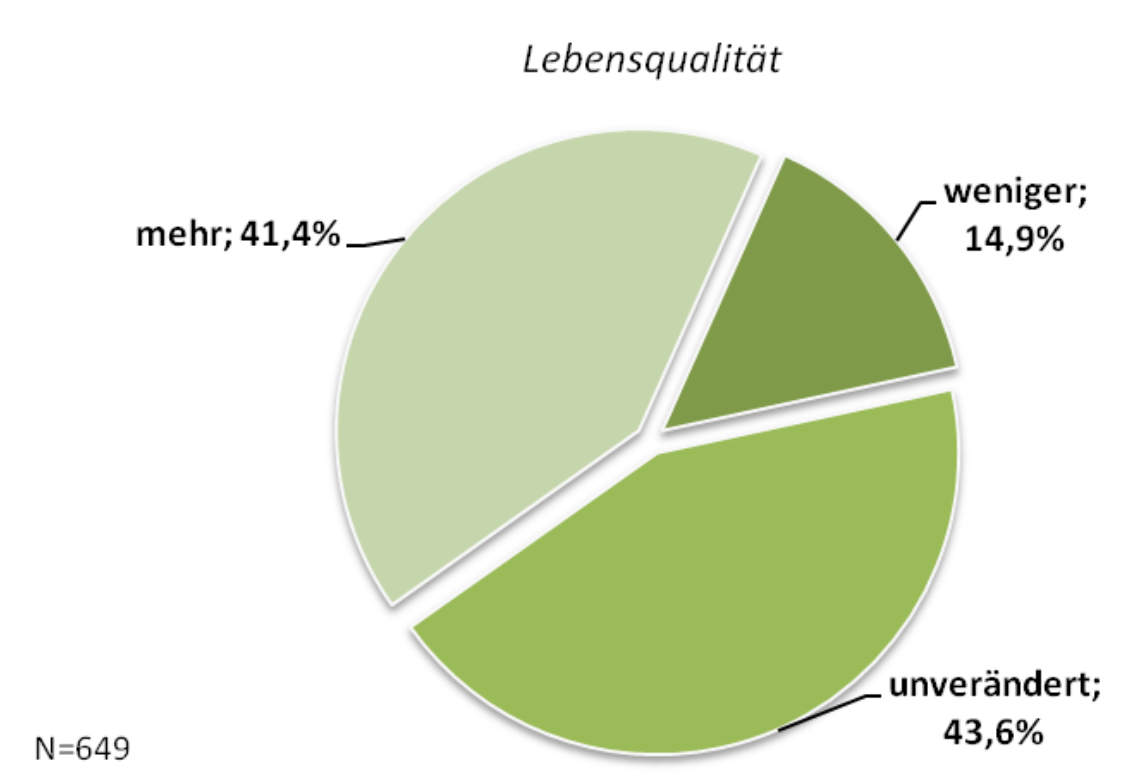




\section{Agrar-Umwelt und Naturschutz}

Die Veränderung bzgl. Lebensqualität wird von jenen Personen, die an Bildungsveranstaltungen der Agrarumweltmaßnahmen teilnahmen, am positivsten eingeschätzt.

Abbildung 72: Lebensqualität - Agrar-Umwelt und Naturschutz

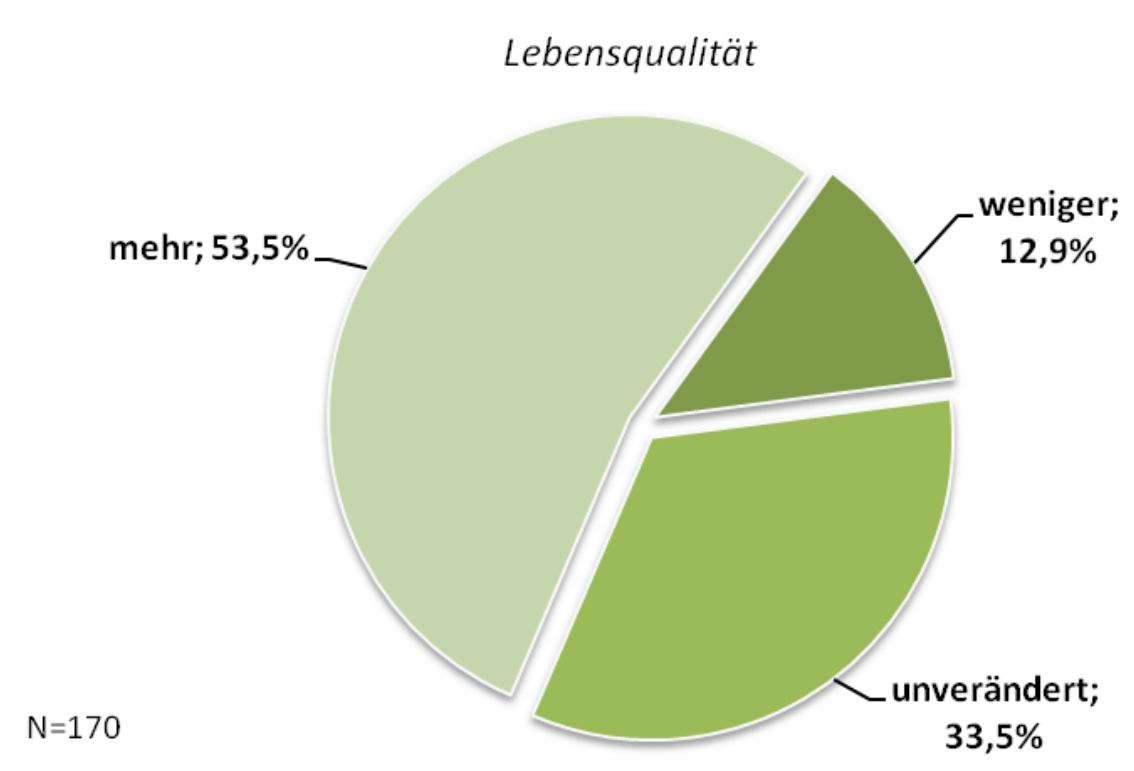

\section{Forstbildungsmaßnahmen}

Die Teilnehmer aus dieser Gruppe wählten, verglichen mit den anderen Bildungsmaßnahmen deutlich seltener die Kategorie "mehr".

Abbildung 73: Lebensqualität - Forstbildungsmaßnahmen

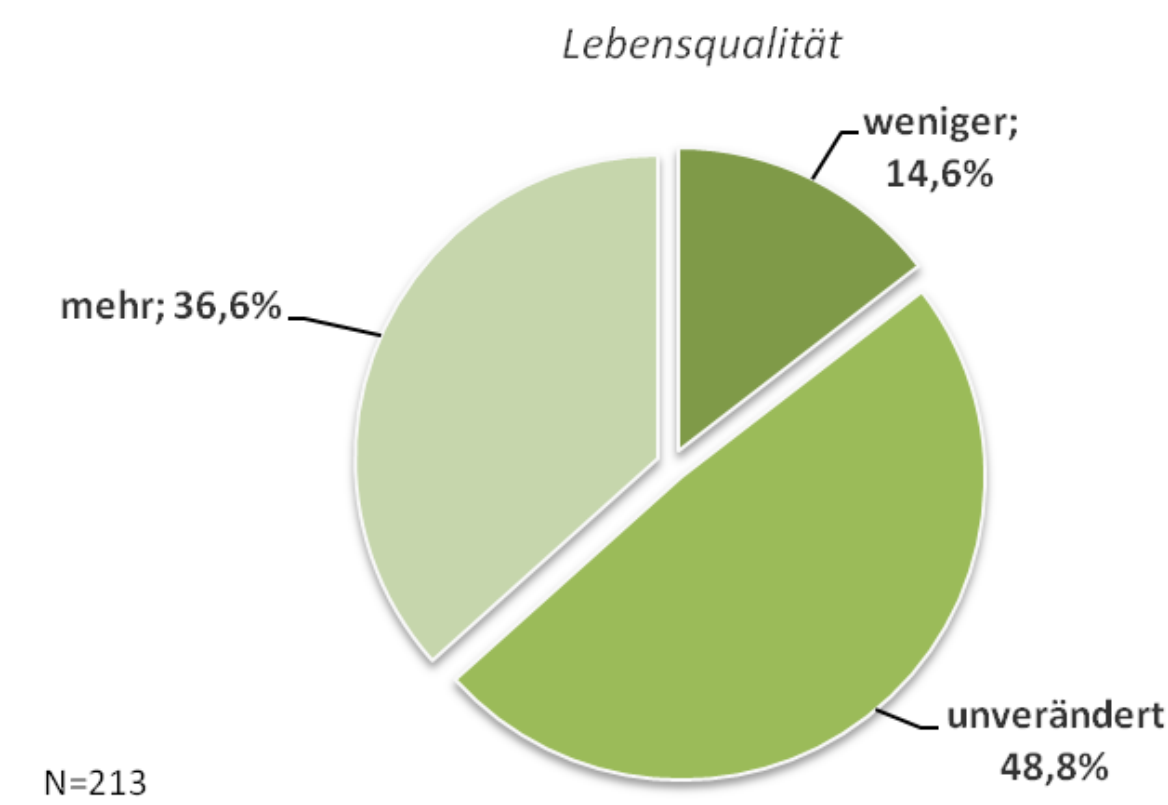




\section{Gender}

Bei der Betrachtung der Genderunterschiede fällt auf, dass Frauen die Veränderung bzgl. Lebensqualität seit 2007 deutlich positiver einschätzen als die männlichen Land- bzw. Forstwirte.

Abbildung 74: Lebensqualität - Gender

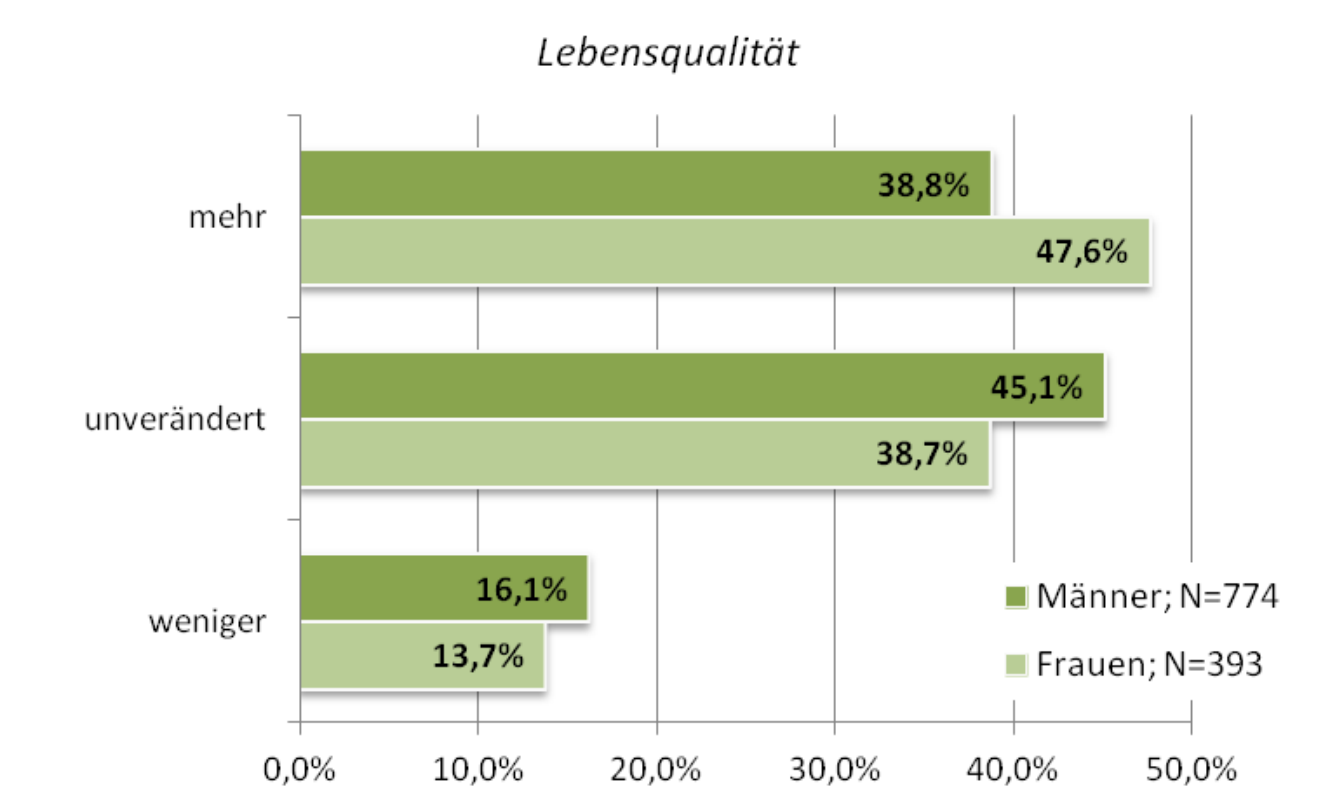

\subsection{Regionale Entwicklung, Wissenstransfer und innovative Ideen}

Bezüglich „Regionale Entwicklung" zeigt die unten stehende Abbildung 75, dass Personen aus der Land- bzw. Forstwirtschaft, die die Möglichkeit einer Aus- und Weiterbildung im Rahmen der LE nutzten, engagierter in nicht-agrarischen, die regionale Entwicklung unterstützenden Bereichen tätig sind. Die Grafik bezieht sich auf die bereits in Kapitel 4.6 behandelte Frage aus der OnlineBefragung "Sind Sie zusätzlich in einem der folgenden Bereiche tätig?".

Abbildung 75: Regionale Entwicklung - nicht-agrarische Bereiche

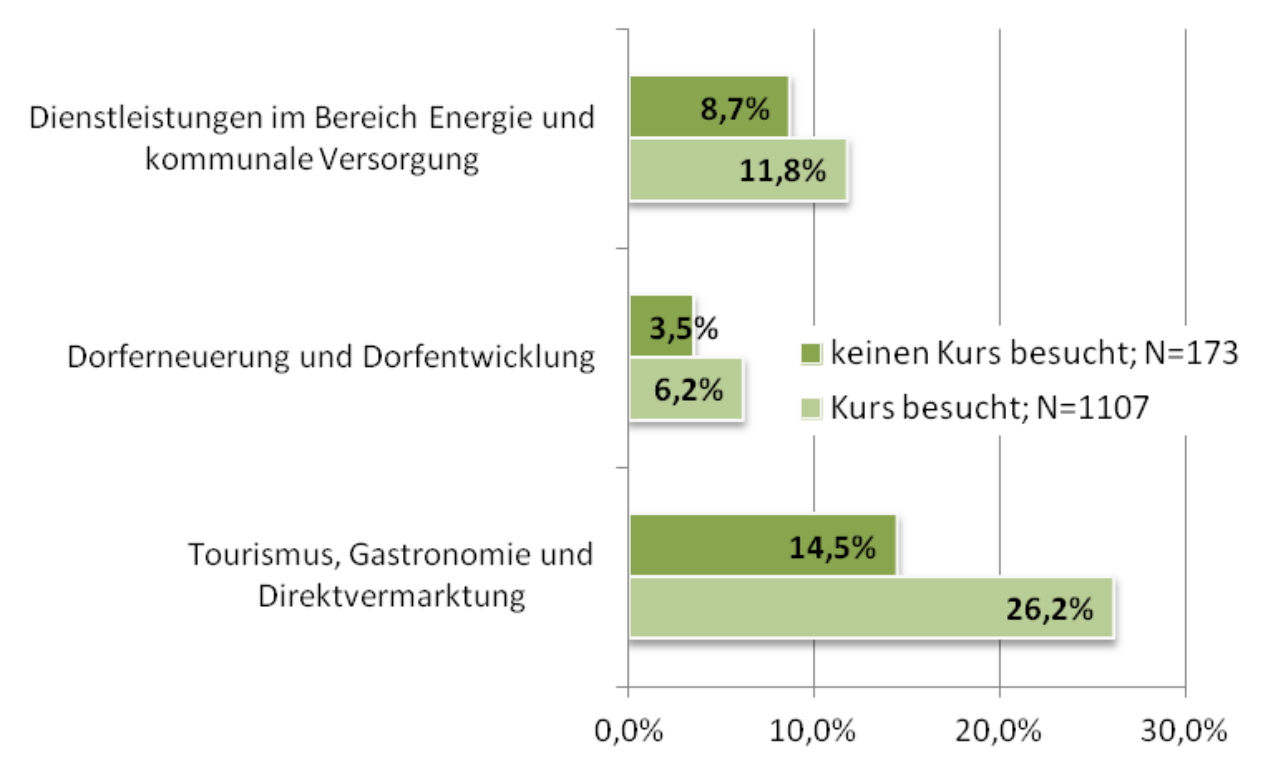


Aus der Auswertung über die seitens der Land- bzw. ForstwirtInnen (gesamt) nachgefragten Themen der Bildungsangebote (Abbildung 76) geht hervor, dass die Themen zum Bereich „Regionale Entwicklung" (in der Grafik zuoberst und in dunkler Farbe angeführt) im Vergleich zu allen anderen weniger nachgefragt werden. Ein Vergleich mit den Abbildungen 100 und 101 (siehe Kapitel 4.11.2) und den entsprechenden Abbildungen in Kapitel 4.4 zeigt auch, dass das Angebot der Bildungsveranstalter zu diesem Thema geringer ist und seitens der Land- bzw. ForstwirtInnen auch ein Wunsch nach einem größeren Angebot dazu besteht.

\section{Abbildung 76: Regionale Entwicklung - Bildungsangebote}

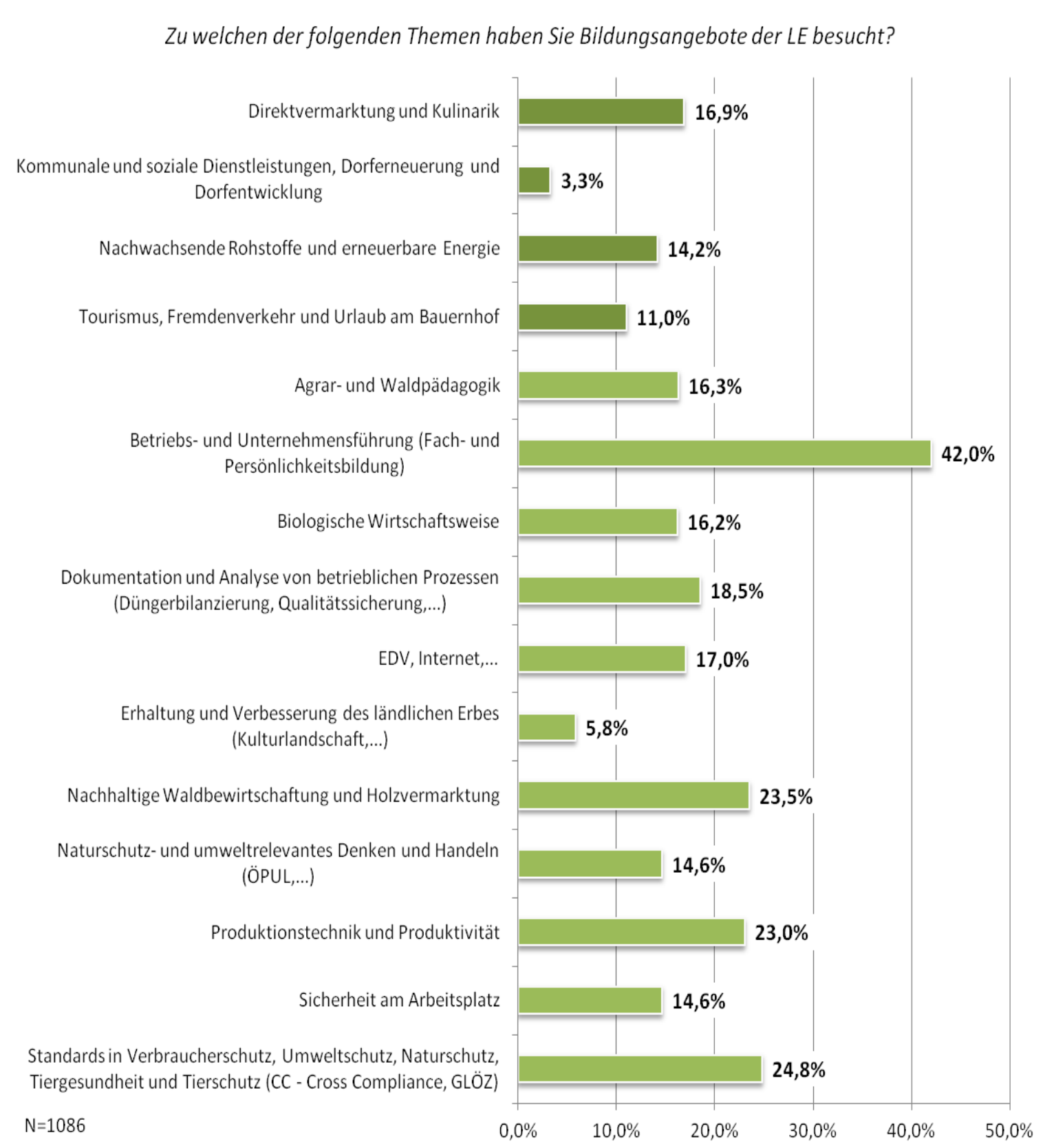


Ein Vergleich ausgewählter, nicht-agrarischer Tätigkeitsfelder zeigt, in welchem Ausmaß Personen, die zu den verschiedenen Bildungsmaßnahmen Kurse besuchten, in den Teilbereichen der regionalen Entwicklung tätig sind.

Abbildung 77: Regionale Entwicklung - Vergleich nach Bildungsmaßnahmen

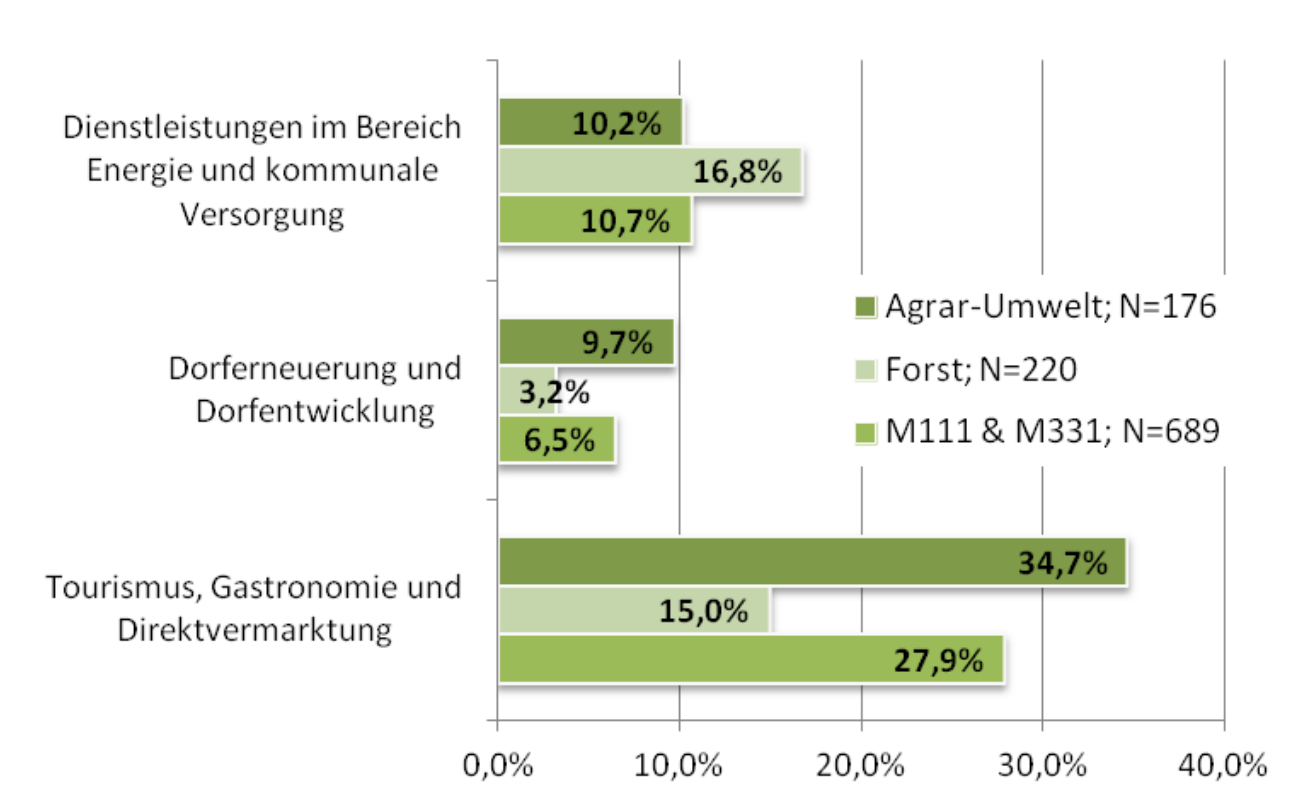

Folgende Aussagen aus den Dialog Interviews behandeln die, in diesem Kapitel betrachteten Themenfelder:

„Durch die Bildungsangebote wird auch der Blickwinkel erweitert."
„Man erhält fachlichen Input von den Referenten."
„Sehr wichtig ist auch der Austausch zwischen den TeilnehmerInnen."

\subsection{Chancengleichheit}

Das Thema Chancengleichheit wurde in den vorhergehenden Kapiteln jeweils bei den Vergleichen der Antworten von Frauen und Männern aus der Land- bzw. Forstwirtschaft beachtet.

Die Beteiligung an Kursen und Veranstaltungen der Ländlichen Entwicklung war bei den befragten Frauen sogar etwas höher als bei den befragten Männern aus der Land- und Forstwirtschaft. 
Abbildung 78: Chancengleichheit - Kursteilnahme

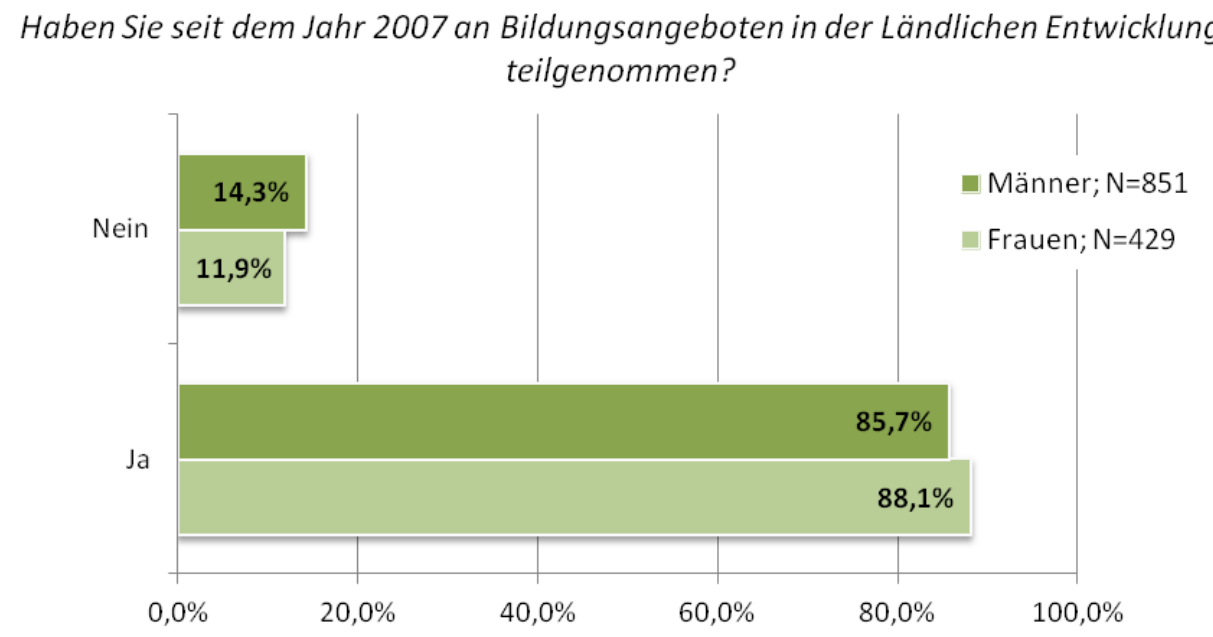

Sehr unterschiedlich sind die Gründe, warum auf eine Teilnahme an Bildungsangeboten der LE verzichtet wurde. Bei Frauen spielt dabei der Faktor Zeit eine wesentlich größere Rolle, als bei den Männern, die den Fragebogen beantworteten. Männer hingegen sehen sich bereits als ausreichend (aus-) gebildet und können keinen Nutzen für die berufliche Tätigkeit erkennen. Hoch ist bei beiden auch der Anteil jener, die angaben, keine Informationen zu den Bildungsangeboten erhalten zu haben. Der Preis für die Kurse und Veranstaltungen spielt sowohl bei den Frauen als auch bei den Männern eine eher unwichtigere Rolle.

Abbildung 79: Chancengleichheit - Gründe für Nicht-Teilnahme

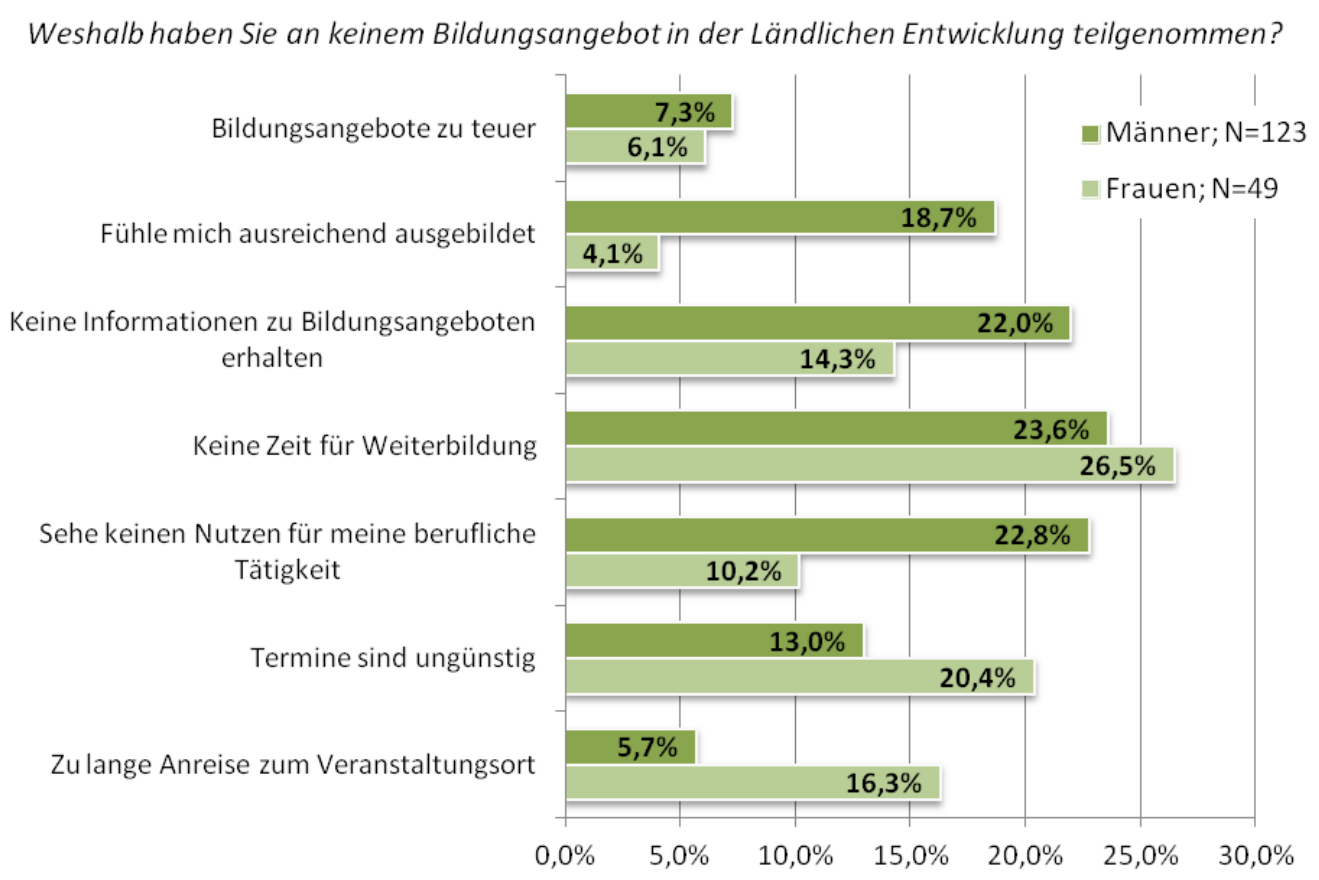

Der Vergleich der Schulbildungen zeigt weitere Unterschiede auf. Von den Personen, die den Fragebogen beantwortet haben, besitzen Land- bzw. Forstwirtinnen zwar häufiger einen Universitätsabschluss oder eine Matura, als die männliche Vergleichsgruppe, gleichzeitig ist bei den Frau- 
en der Anteil jener, die über einen Pflichtschulabschluss verfügen, deutlich höher als bei den Land- bzw. Forstwirten. Bei den Männern gaben daneben wesentlich mehr an, die Meisterprüfung absolviert zu haben.

Abbildung 80: Schulbildung nach Gender - Frauen

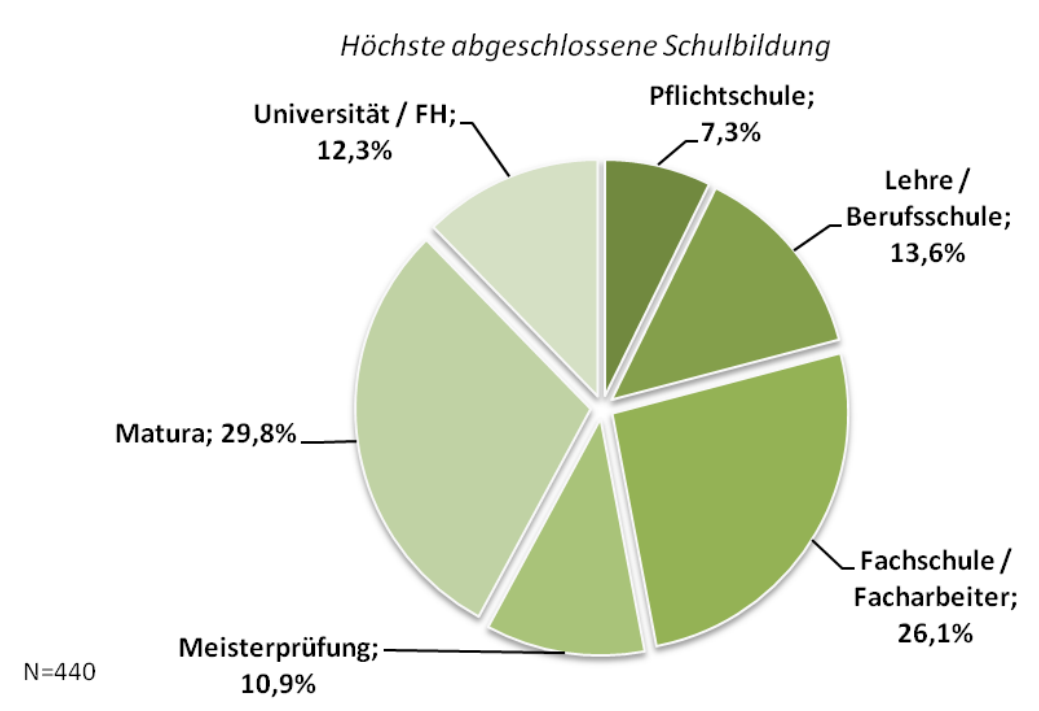

Abbildung 81: Schulbildung nach Gender - Männer

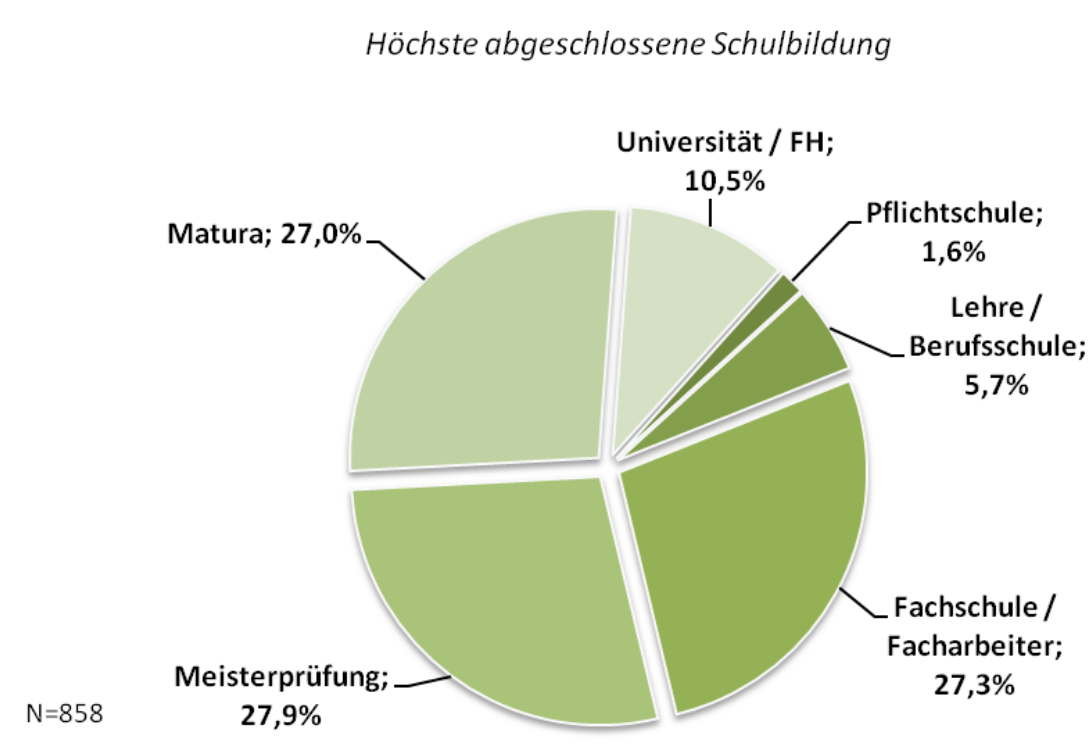

Bezüglich Chancengleichheit wurden in den Dialog Interviews folgende Wünsche und Anregungen geäußert:

"Gleichstellung von Frauen und Männern ist wichtig. Es sollte der Frage nachgegangen werden, warum Frauen zu bestimmten Veranstaltungen nicht kommen. 42\% der Hofführer sind weiblich! Das sollte sich in den Teilnehmer-Zahlen widerspiegeln!"

„Speziell Frauen ansprechen, welche Bildungsmaßnahmen für sie interessant wären." 
„Es wäre wichtig, frauenspezifische Bildungsprojekte gefördert zu bekommen. Ein Projekt nur für Frauen wäre nicht gefördert worden und musste auch für Männer geöffnet werden."

„Frauen-Kurse ermöglichen Lernen / Austausch in einer sicheren Umgebung."

\subsection{Weitere Analyseresultate}

Zusätzlich zu den vom Auftraggeber in der Ausschreibung formulierten Fragestellungen ergaben sich im Laufe der Evaluierung weitere Fragestellungen, auf die in diesem Kapitel näher eingegangen werden soll. Die Auswertungen beleuchten dabei weitere Zielgruppen des Fragebogens. Einerseits wird die Gruppe der Seminarbäuerinnen, Schule-am-Bauernhof BetreiberInnen und WaldpädagogInnen näher betrachtet, andererseits wird der Fragebogen hinsichtlich der Gruppe der Bildungsveranstalter ausgewertet und schließlich wird auf jene TeilnehmerInnen an der Online-Befragung näher eingegangen, die ebenfalls Kurse der LE absolviert haben, jedoch nicht in der Land- bzw. Forstwirtschaft tätig sind.

\subsubsection{Seminarbäuerinnen, Schule-am-Bauernhof Betriebe, WaldpädagogInnen}

299 Personen, die an der Online-Befragung teilnahmen gaben an, im agrar- und waldpädagogischen Bereich tätig zu sein. Die genaue Aufteilung in die verschiedenen Tätigkeitsbereiche ist in Abbildung 82 dargestellt.

In den Dialog Interviews wurden folgende Anmerkungen zur Agrar- und WaldpädagogikAusbildung gemacht:

„Alle 2-3 Jahre wäre ein Austausch mit anderen Anbietern von Schule am Bauernhof wichtig."

„Ranger-Austausch zwischen Lehrgängen verschiedener Nationalparks ist möglich geworden."

Abbildung 82: Aufteilung Agrar- und Waldpädagogik

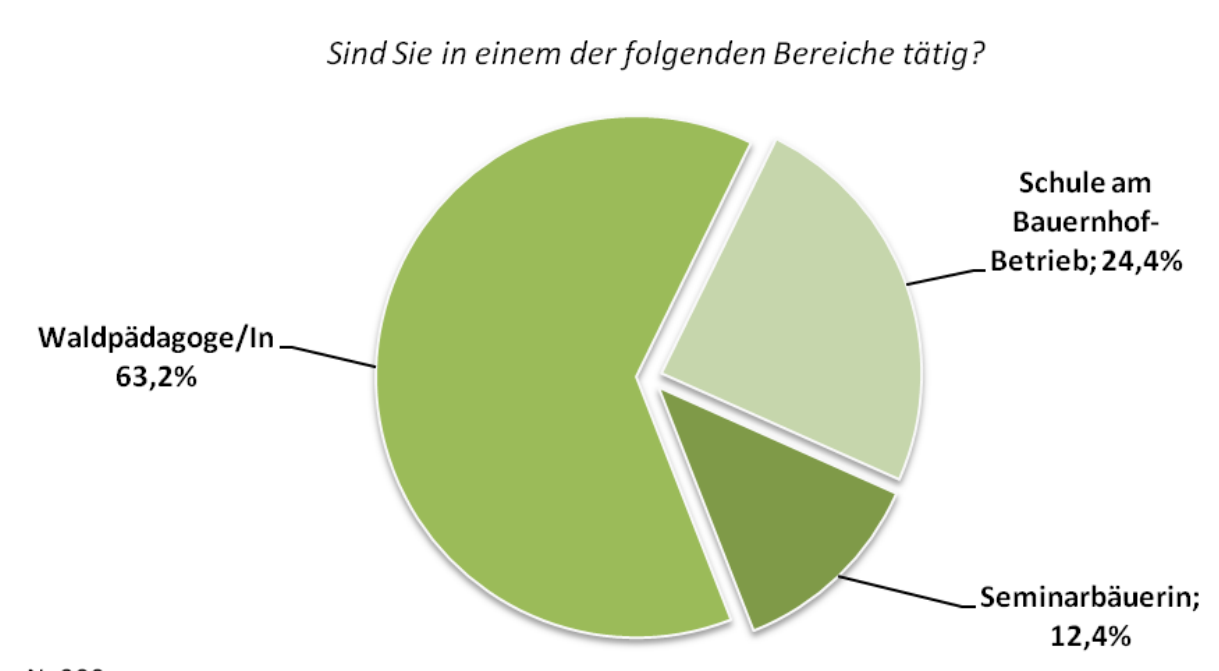

$\mathrm{N}=299$ 
Der Großteil der Agrar- und WaldpädagogInnen kommt aus land- und forstwirtschaftlichen Betrieben. Der Rest stammt zu einem großen Teil aus anderen, nicht-land- oder forstwirtschaftlichen Berufen.

Abbildung 83: Aufteilung nach Betrieb / Organisation - Agrar- und Waldpädagogik In welchem Betrieb bzw. in welcher Organisation sind Sie tätig?

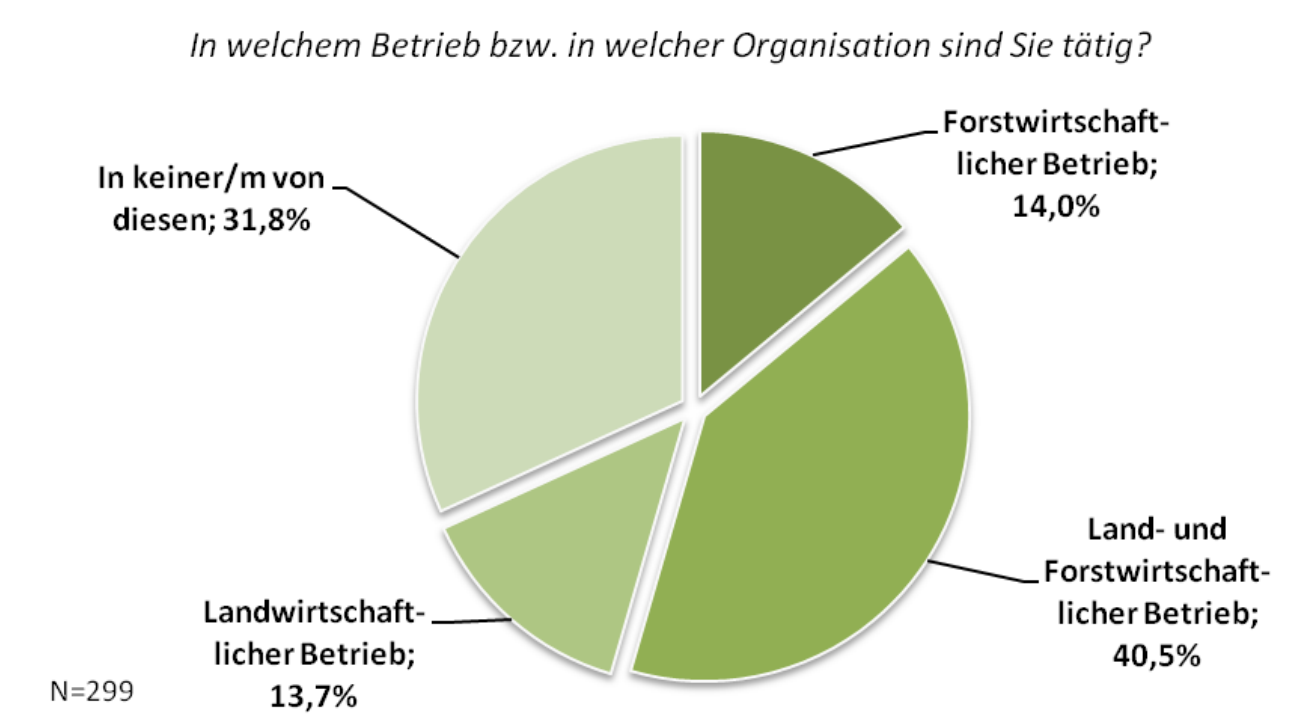

Jene Personen, die in der Kategorie „In keiner/m von diesen“ antworteten, sind fast zur Gänze als Waldpädagogen tätig.

Abbildung 84: Aufteilung Agrar- \& Waldpädagogik - TeilnehmerInnen aus anderen Branchen Sind Sie in einem der folgenden Bereiche tätig?

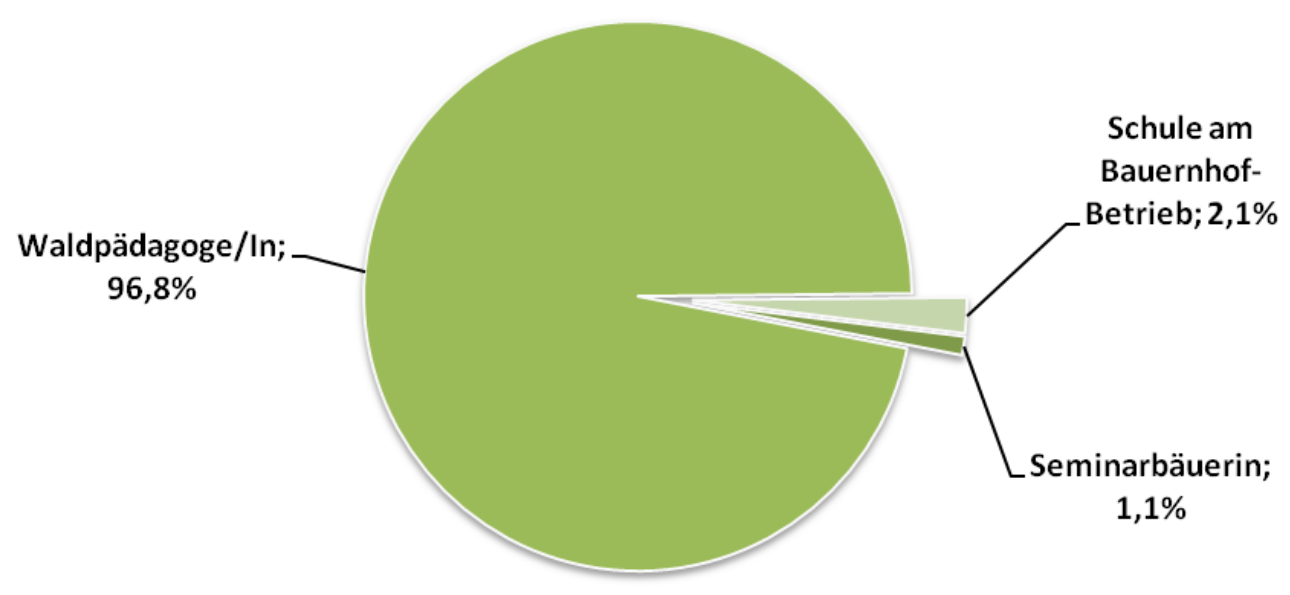

$\mathrm{N}=95$

In weiterer Folge wurde die Personengruppe der Agrar- und Waldpädagogen in der OnlineUmfrage noch speziell zur agrar- und waldpädagogischen Ausbildung befragt. Die Bewertung ist dabei durchwegs sehr positiv. Die mit Abstand meisten negativen Bewertungen erhielt die Aussage, dass die Ausbildung alle relevanten Inhalte umfasst. 
Abbildung 85: Empfundener Nutzen - Agrar- und WaldpädagogInnen gesamt

Stimmen Sie den folgenden Aussagen über die Ausbildung zur/m Seminarbäuerin / Waldpädagogen oder zur Schule am Bauernhof Betreiberin zu?

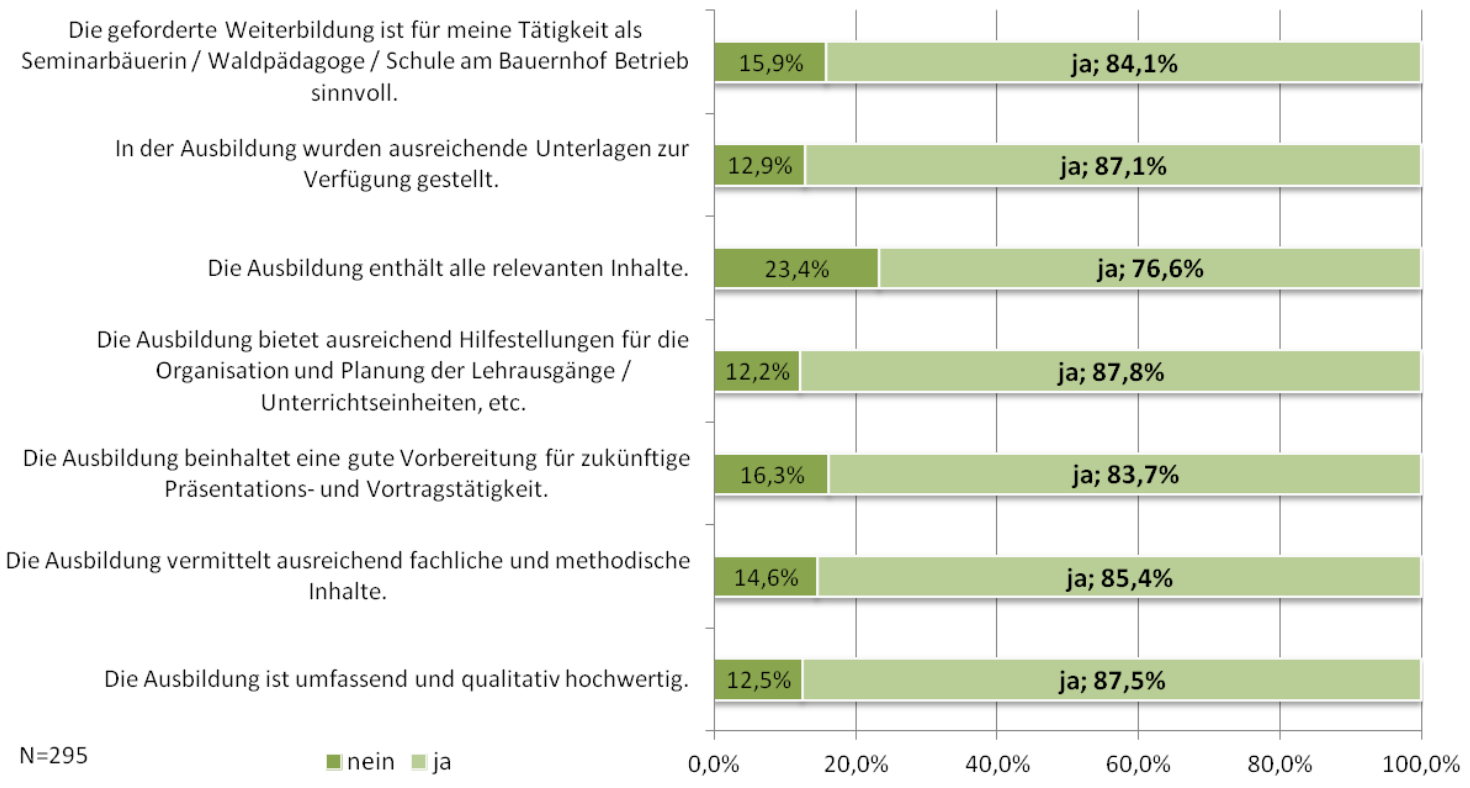

Diese Frage zur Bewertung der Agrar- und Waldpädagogischen Ausbildung wird nun differenzierter, zunächst jeweils getrennt für die Seminarbäuerinnen, die WaldpädagogInnen und die Schule am Bauernhof-Betriebe, und später für Land- bzw. ForstwirtInnen sowie TeilnehmerInnen aus nicht-agrarischen Berufen betrachtet.

\section{Abbildung 86: Empfundener Nutzen - Seminarbäuerinnen}

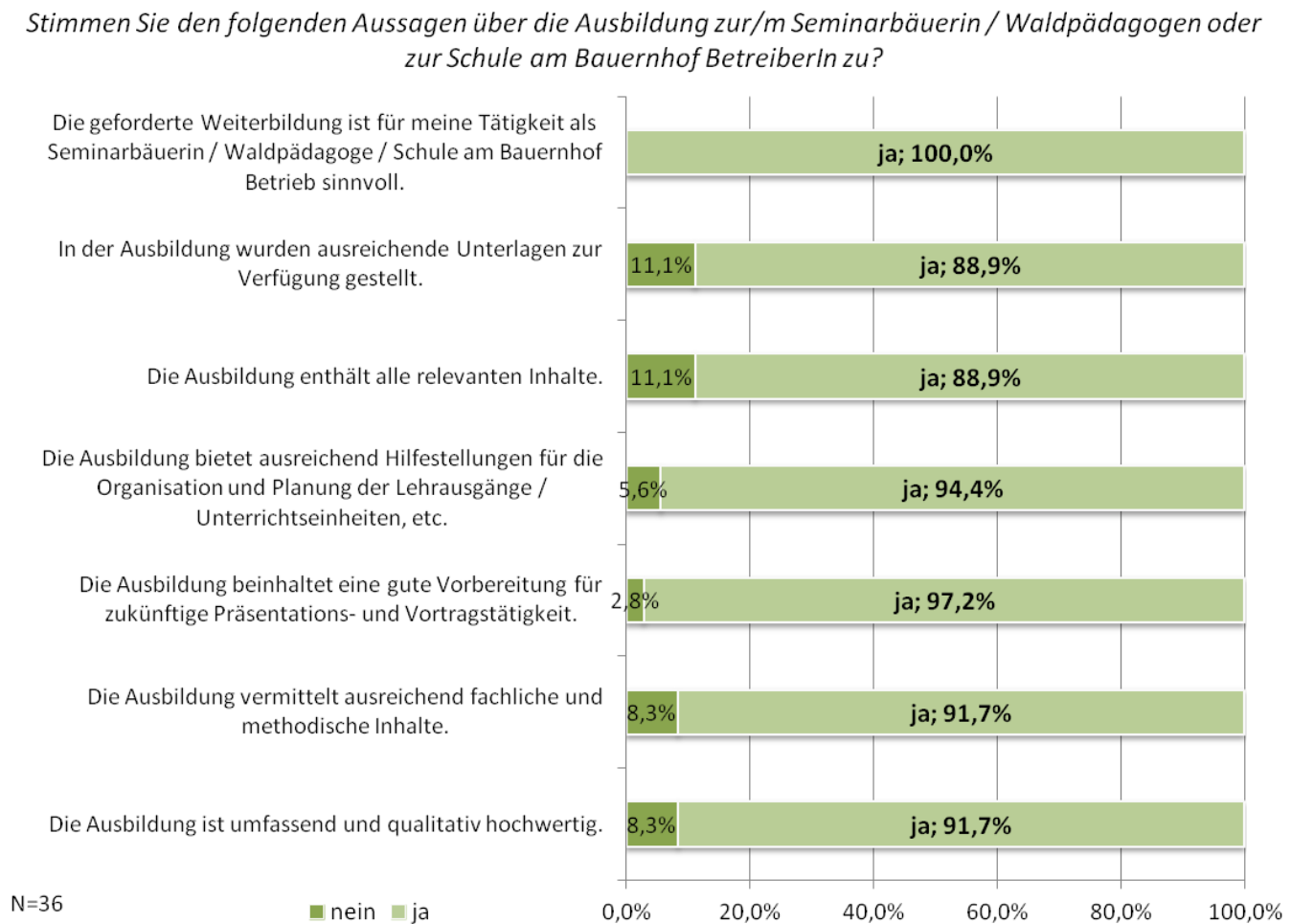


Abbildung 87: Empfundener Nutzen - WaldpädagogInnen

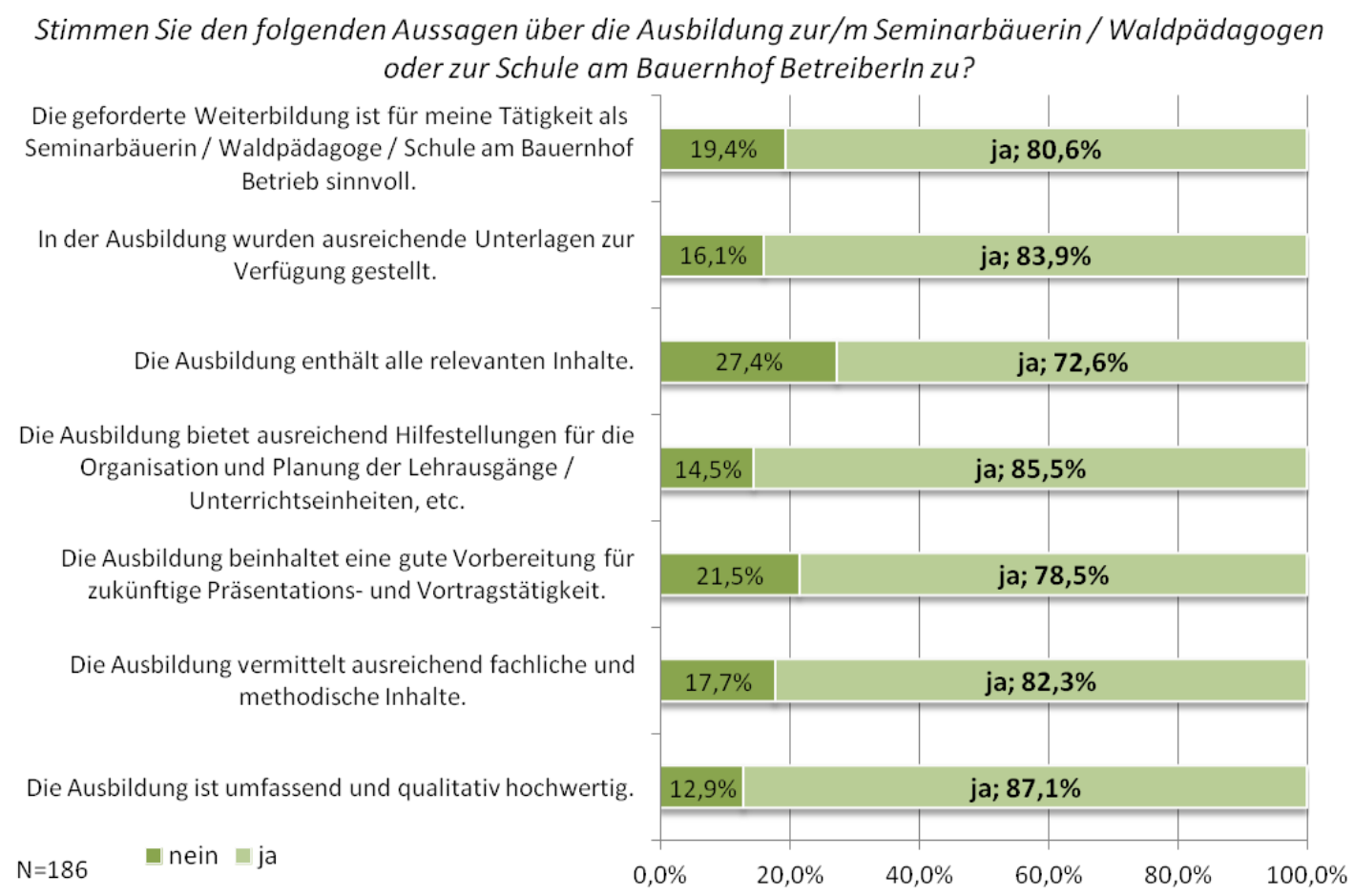

Abbildung 88: Empfundener Nutzen - Schule am Bauernhof-Betriebe

\section{Stimmen Sie den folgenden Aussagen über die Ausbildung zur/m Seminarbäuerin / Waldpädagogen oder} zur Schule am Bauernhof Betreiberln zu?

Die geforderte Weiterbildung ist für meine Tätigkeit als Seminarbäuerin / Waldpädagoge / Schule am Bauernhof Betrieb sinnvoll.

In der Ausbildung wurden ausreichende Unterlagen zur Verfügung gestellt.

Die Ausbildung enthält alle relevanten Inhalte.

Die Ausbildung bietet ausreichend Hilfestellungen für die Organisation und Planung der Lehrausgänge / Unterrichtseinheiten, etc.

Die Ausbildung beinhaltet eine gute Vorbereitung für zukünftige Präsentations- und Vortragstätigkeit.

Die Ausbildung vermittelt ausreichend fachliche und methodische Inhalte.

Die Ausbildung ist umfassend und qualitativ hochwertig.
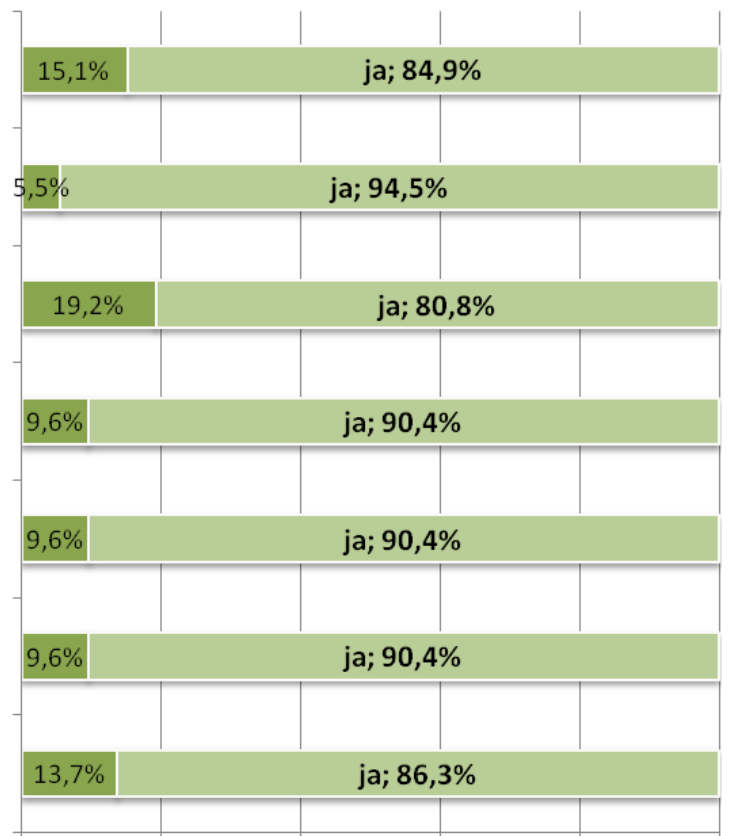

$\mathrm{N}=73 \quad$ nein $\mathbf{a j a}$

$0,0 \%$

$20,0 \%$

$40,0 \%$

$60,0 \%$

$80,0 \%$

$100,0 \%$ 


\section{Abbildung 89: Empfundener Nutzen - Land- bzw. ForstwirtInnen}

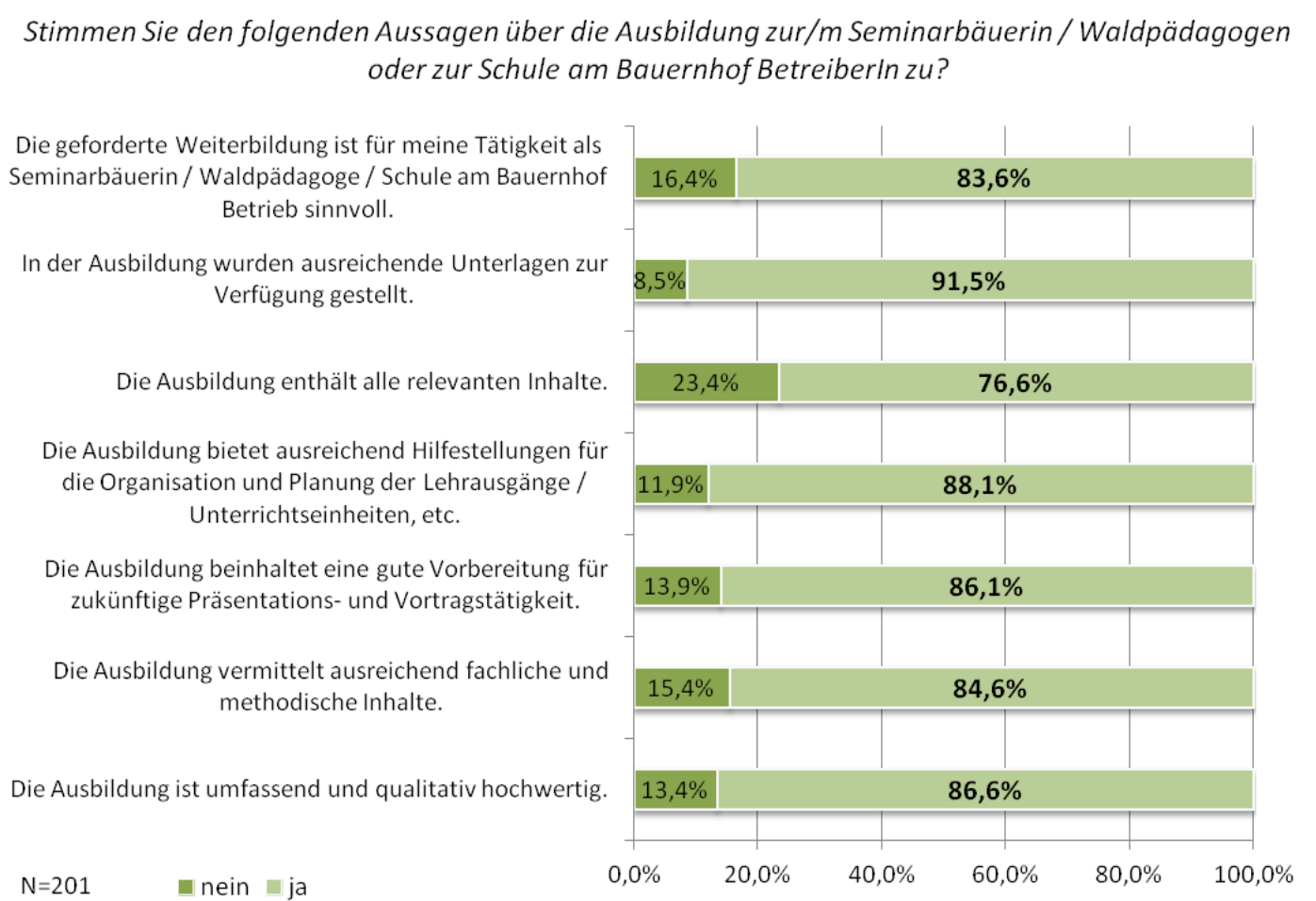

Abbildung 90: Empfundener Nutzen - Teilnehmerlnnen aus anderen Branchen

Stimmen Sie den folgenden Aussagen über die Ausbildung zur/m Seminarbäuerin / Waldpädagogen oder zur Schule am Bauernhof Betreiberin zu?

Die geforderte Weiterbildung ist für meine Tätigkeit als Seminarbäuerin / Waldpädagoge / Schule am Bauernhof Betrieb sinnvoll.

In der Ausbildung wurden ausreichende Unterlagen zur Verfügung gestellt.

Die Ausbildung enthält alle relevanten Inhalte.

Die Ausbildung bietet ausreichend Hilfestellungen für die Organisation und Planung der Lehrausgänge / Unterrichtseinheiten, etc.

Die Ausbildung beinhaltet eine gute Vorbereitung für zukünftige Präsentations- und Vortragstätigkeit.

Die Ausbildung vermittelt ausreichend fachliche und methodische Inhalte.

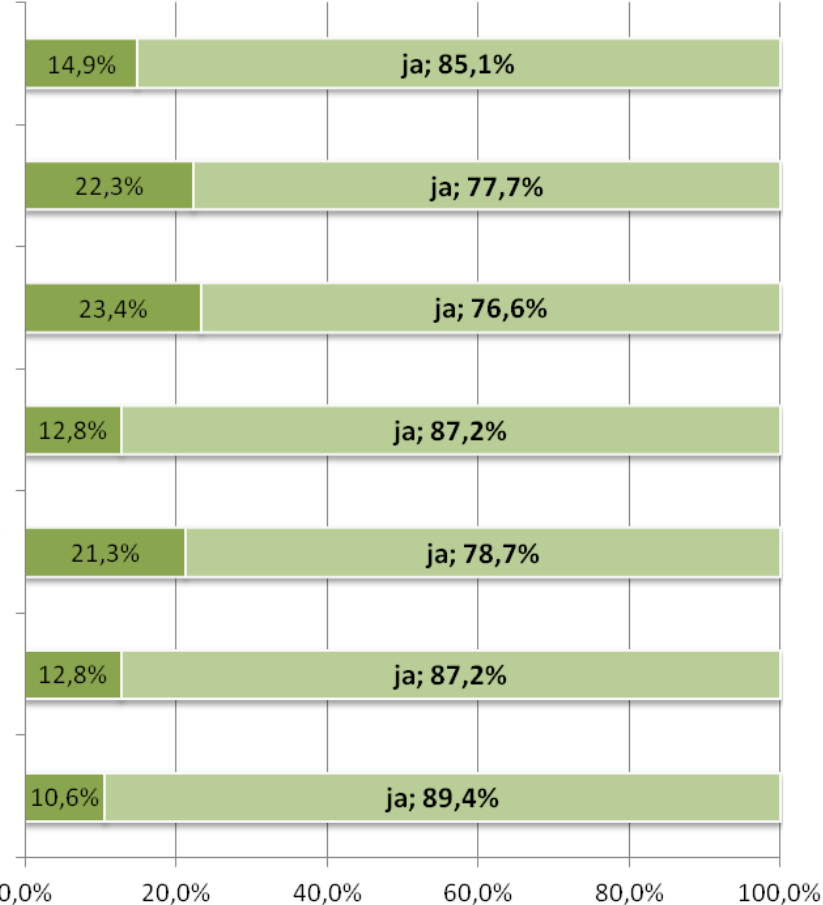


Neben Kursen zu Agrar- und Waldpädagogik besuchte diese Gruppe der Befragten noch weitere Kurse und Bildungsveranstaltungen der Ländlichen Entwicklung (siehe Abbildung 91).

Abbildung 91: besuchte Kurse - Agrar- und Waldpädagogen

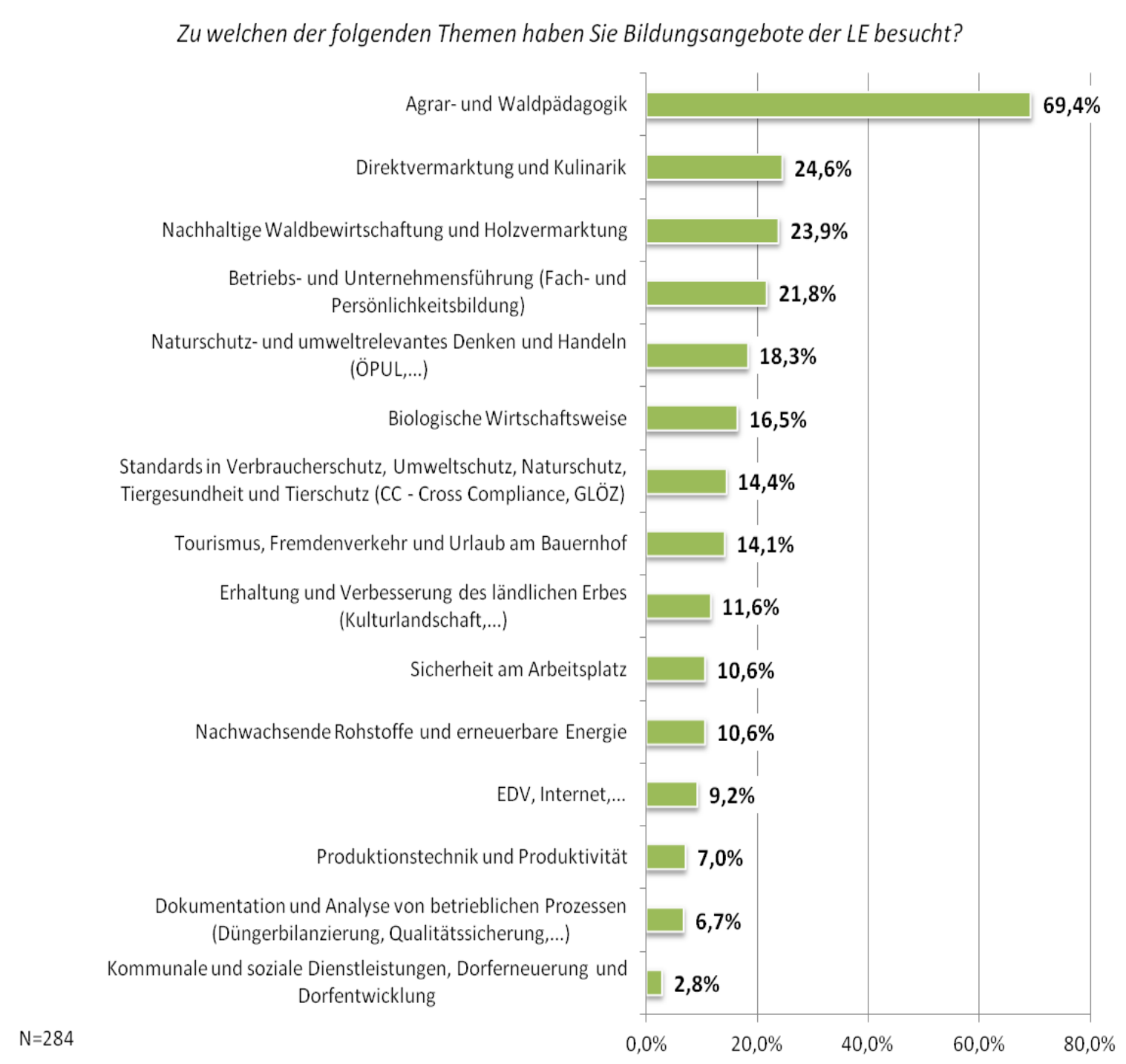

Befragt zu den Veränderungen seit 2007 sehen die meisten der Agrar- und WaldpädagogInnen eine persönliche Verbesserung bzw. Steigerung hinsichtlich Naturschutz und Umwelt. 
Abbildung 92: Veränderungen seit 2007- Agrar- und Waldpädagogen

Was ist für Sie auf Ihrem land- und forstwirtschaftlichen Betrieb bzw. im Ländlichen Raum seit 2007 anders?

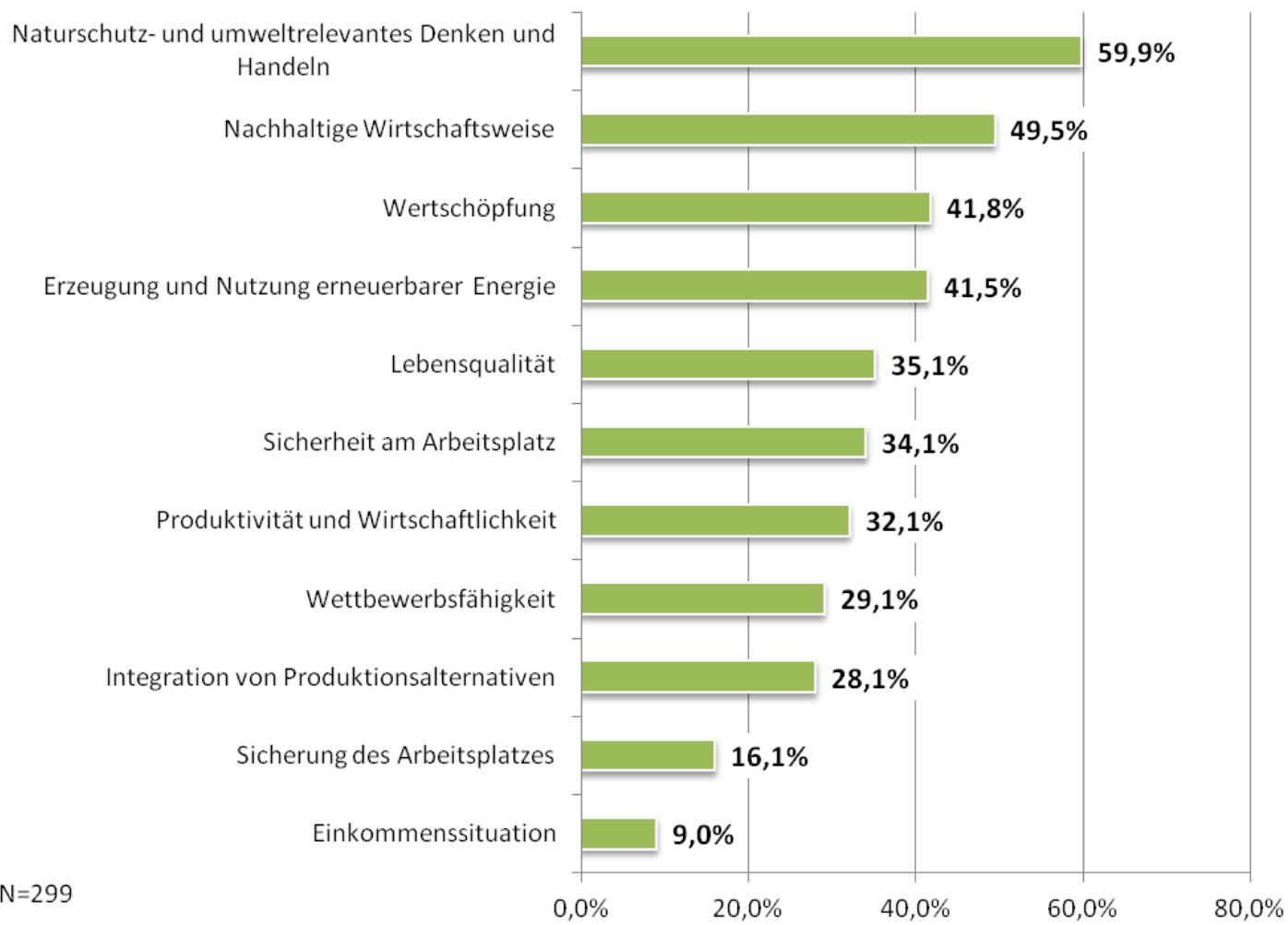

\subsubsection{Bildungsveranstalter}

Seitens der Bildungsveranstalter beantworteten insgesamt 259 Frauen und 392 Männer den Fragebogen. Der Großteil (fast drei Viertel) dieser Gruppe gab an, im Rahmen des laufenden Programms der Ländlichen Entwicklung Fördergelder erhalten zu haben.

\section{Abbildung 93: Anteil der Veranstalter mit erhaltener Veranstalterförderung}

Erhielt Ihre Organisation Veranstalterförderung im Rahmen der Ländlichen Entwicklung 2007-2013?

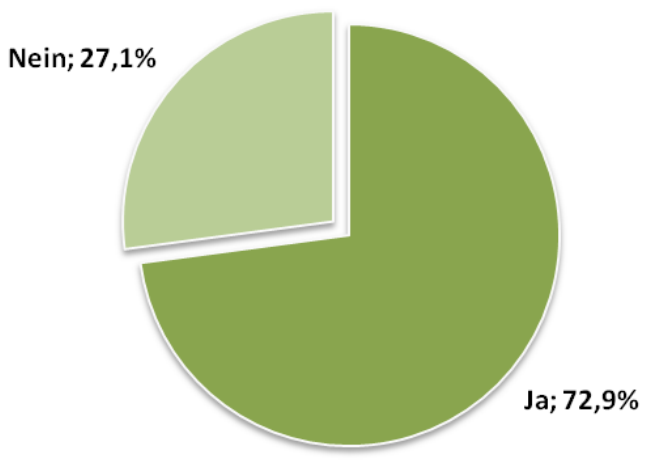


Die meiste Zeit wenden die Bildungsveranstalter für die "Vorbereitung, Durchführung und Nachbearbeitung" von Bildungs- und Informationsmaßnahmen auf. Die "Förderungsabwicklung" ist ebenfalls sehr aufwändig für die befragte Gruppe der Veranstalter. Mit der Erstellung von Bedarfsstudien beschäftigen sich fast alle Untergruppen aus dem Kollektiv der Veranstalter (ausgenommen Natur- und Umweltschutz) am wenigsten. Neben der Darstellung der zusammengefassten Antworten aller befragten Veranstalter (Abbildung 94), werden nachfolgend die einzelnen Veranstalter separat betrachtet.

Abbildung 94: Arbeitsaufwand nach Tätigkeit - Veranstalter gesamt

Wie viel Arbeitszeit wenden Sie für die folgenden Tätigkeiten jeweils auf?

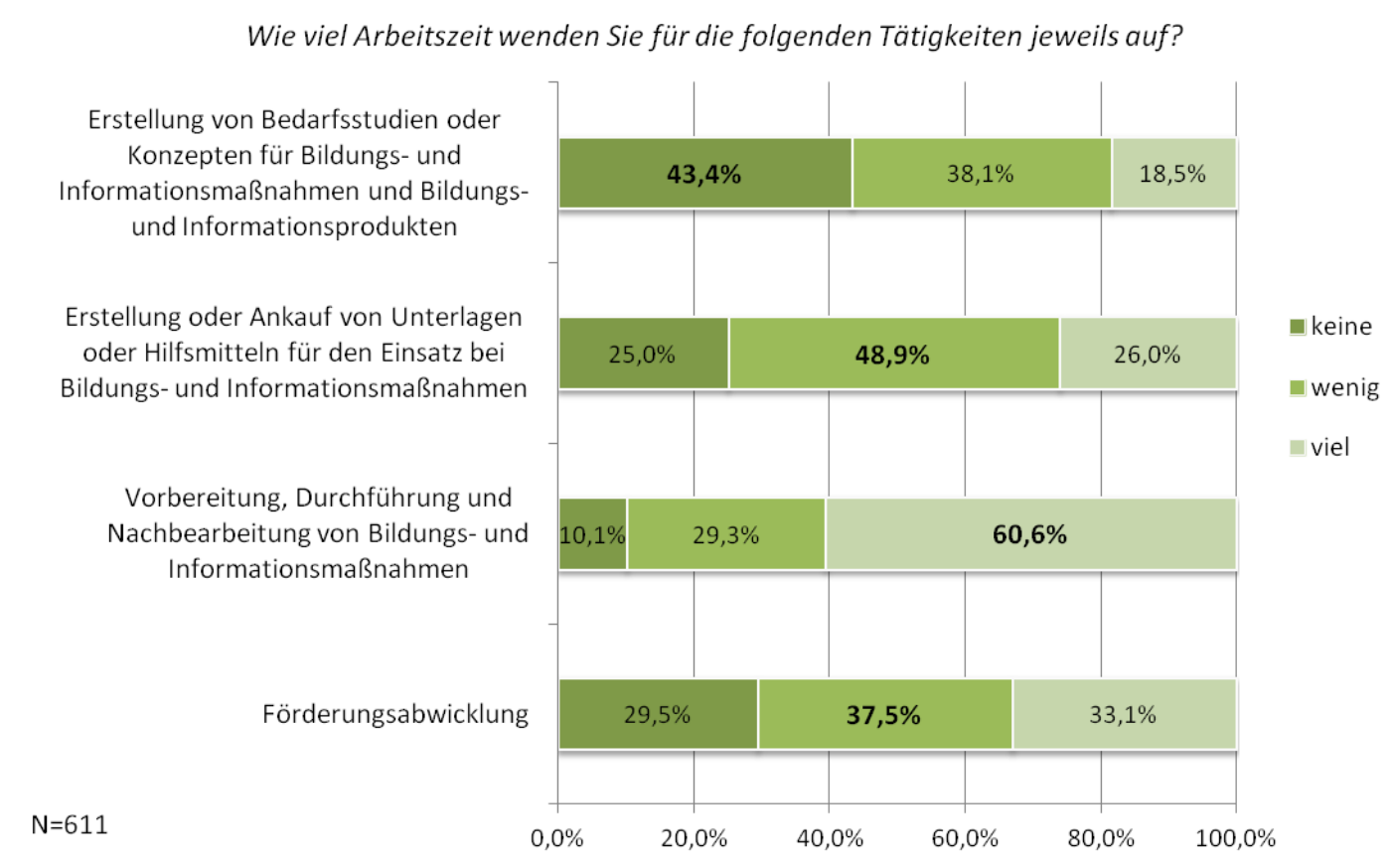

Abbildung 95: Arbeitsaufwand nach Tätigkeit - FAST

Wie viel Arbeitszeit wenden Sie für die folgenden Tätigkeiten jeweils auf?

Erstellung von Bedarfsstudien oder Konzepten für Bildungs- und Informationsmaßnahmen und Bildungsund Informationsprodukten

Erstellung oder Ankauf von Unterlagen oder Hilfsmitteln für den Einsatz bei Bildungs- und Informationsmaßnahmen

Vorbereitung, Durchführung und Nachbearbeitung von Bildungs-und Informationsmaßnahmen

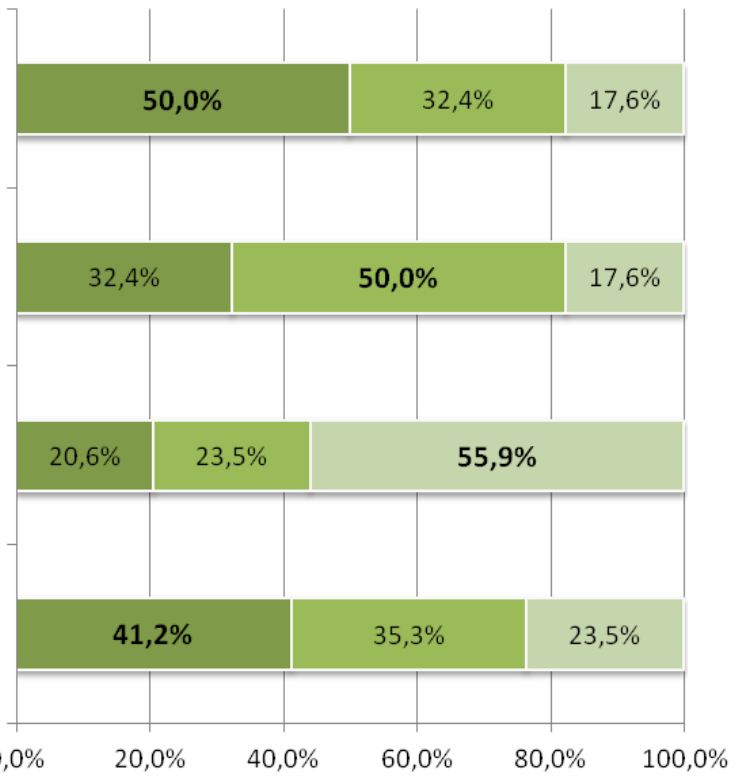


Abbildung 96: Arbeitsaufwand nach Tätigkeit - LFI

Wie viel Arbeitszeit wenden Sie für die folgenden Tätigkeiten jeweils auf?

Erstellung von Bedarfsstudien oder Konzepten für Bildungs- und Informationsmaßnahmen und Bildungs- und Informationsprodukten

Erstellung oder Ankauf von Unterlagen oder Hilfsmitteln für den Einsatz bei Bildungs- und Informationsmaßnahmen

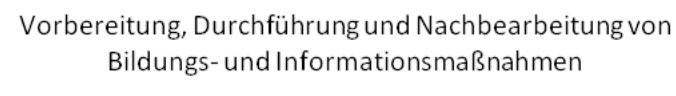
Bildungs- und Informationsmaßnahmen
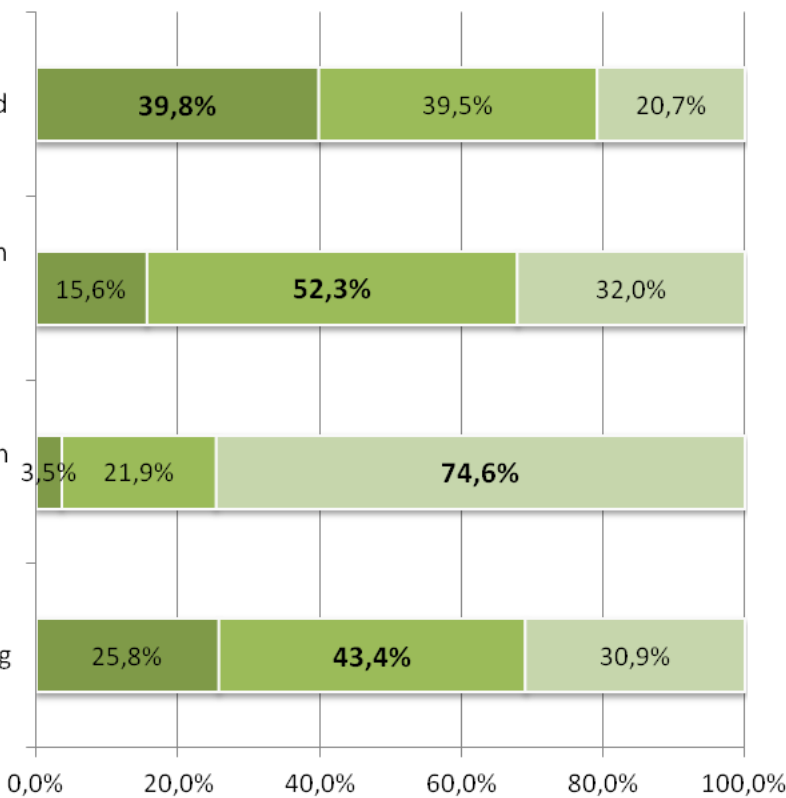

\section{Abbildung 97: Arbeitsaufwand nach Tätigkeit - Natur- \& Umweltschutz}

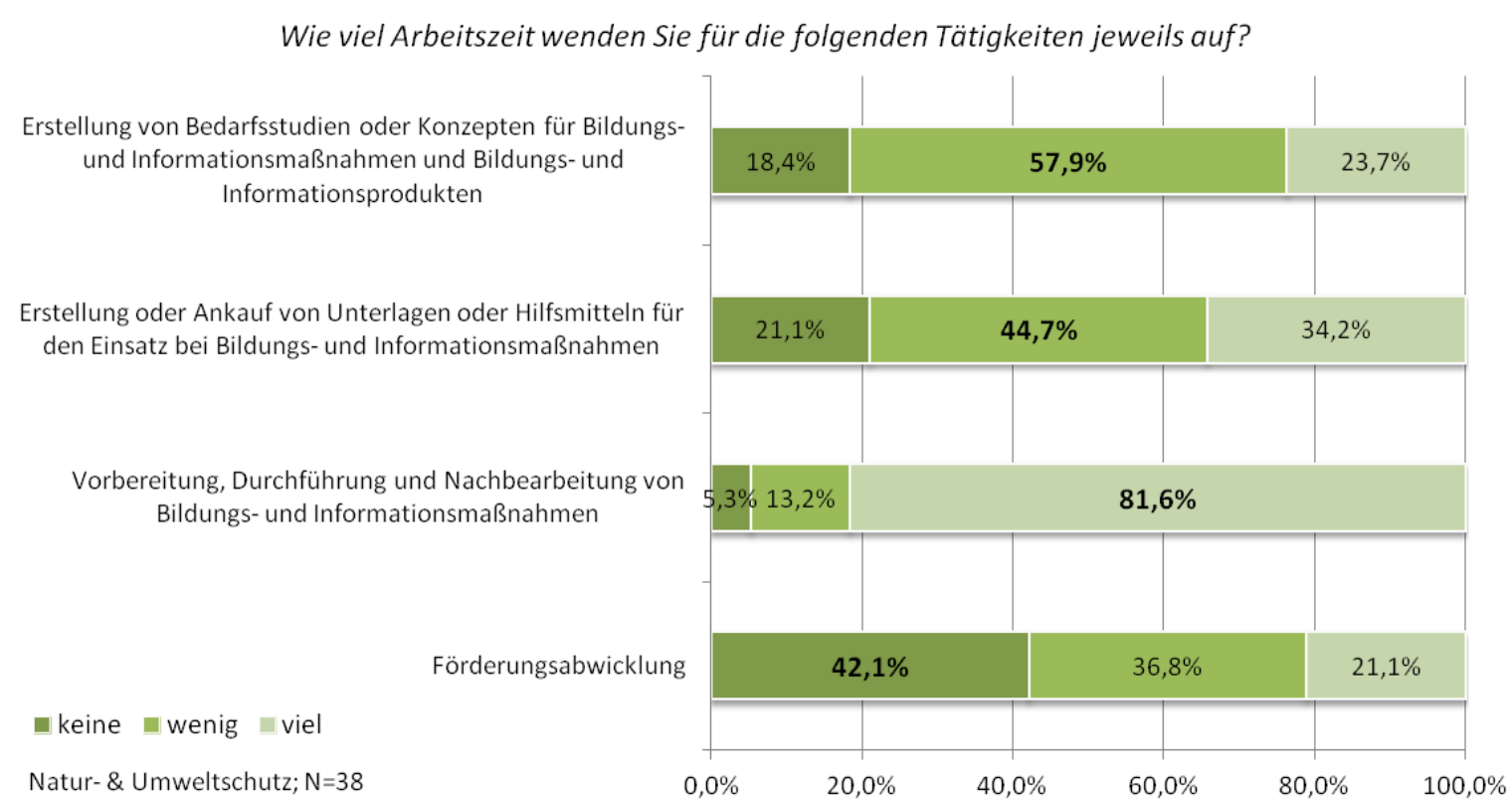


Abbildung 98: Arbeitsaufwand nach Tätigkeit - öffentliche Dienststellen

Wie viel Arbeitszeit wenden Sie für die folgenden Tätigkeiten jeweils auf?

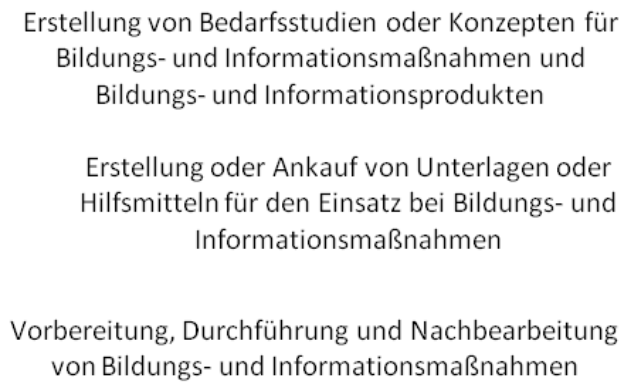

Erstellung von Bedarfsstudien oder Konzepten für Bildungs- und Informationsmaßnahmen und Bildungs- und Informationsprodukten

Erstellung oder Ankauf von Unterlagen oder Hilfsmitteln für den Einsatz bei Bildungs- und Informationsmaßnahmen

Vorbereitung, Durchführung und Nachbearbeitung von Bildungs- und Informationsmaßnahmen

keine wenig viel

Öffentliche Dienststelle; $\mathrm{N}=225$

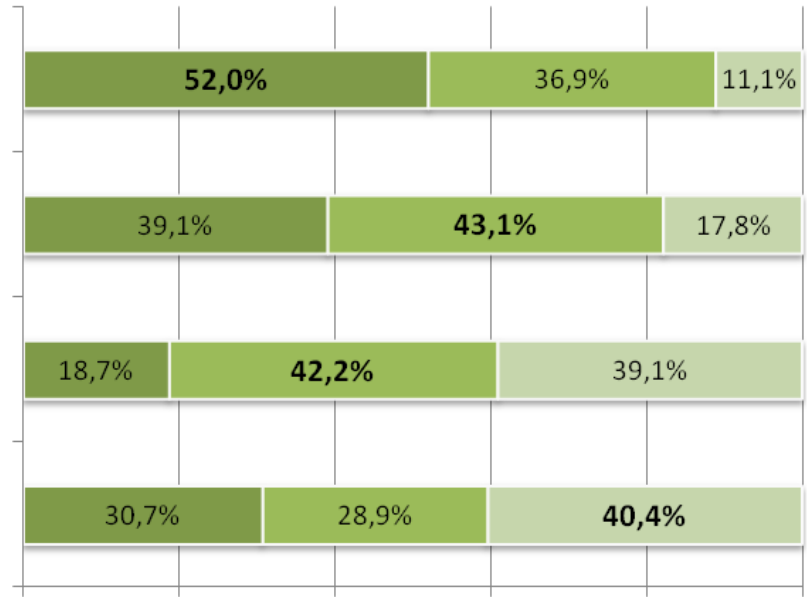

$0,0 \%$

$40,0 \%$

$60,0 \%$

$80,0 \%$

\section{Abbildung 99: Arbeitsaufwand nach Tätigkeit - Sonstige Bildungsveranstalter}

Wie viel Arbeitszeit wenden Sie für die folgenden Tätigkeiten jeweils auf?

Erstellung von Bedarfsstudien oder Konzepten für Bildungsund Informationsmaßnahmen und Bildungs- und

Informationsprodukten

Erstellung oder Ankauf von Unterlagen oder Hilfsmitteln für den Einsatz bei Bildungs- und Informationsmaßnahmen

Vorbereitung, Durchführung und Nachbearbeitung von Bildungs- und Informationsmaßnahmen

sonstige Bildungsveranstalter; $\mathrm{N}=58$

Förderungsabwicklung

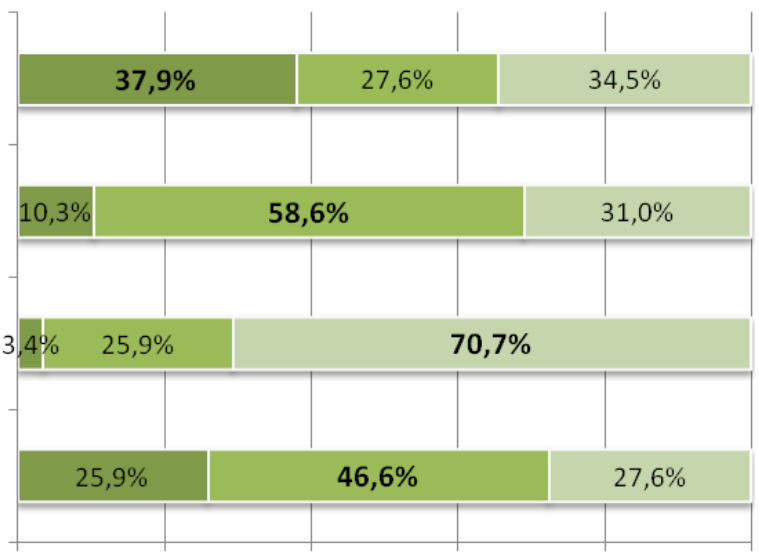

$\begin{array}{lllll}0,0 \% & 20,0 \% & 40,0 \% & 60,0 \% & 80,0 \%\end{array} \quad 100,0 \%$

Bei der Gegenüberstellung der Bewertungen des Bildungsangebotes der Veranstalter mit den Bewertungen der Land- bzw. ForstwirtInnen ist ersichtlich, dass seitens der Veranstalter durchgehend öfter die Kategorie „zu gering“ gewählt wurde als bei den Land- bzw. ForstwirtInnen. 


\section{Abbildung 100a: Bewertung des Bildungsangebote (absolut) - Vergleich Veranstalter / öffentliche Dienststellen vs. $L W / F W$}

Wie sind Ihrer Einschätzung nach die folgenden Themen im Bildungsangebot der LE vertreten?

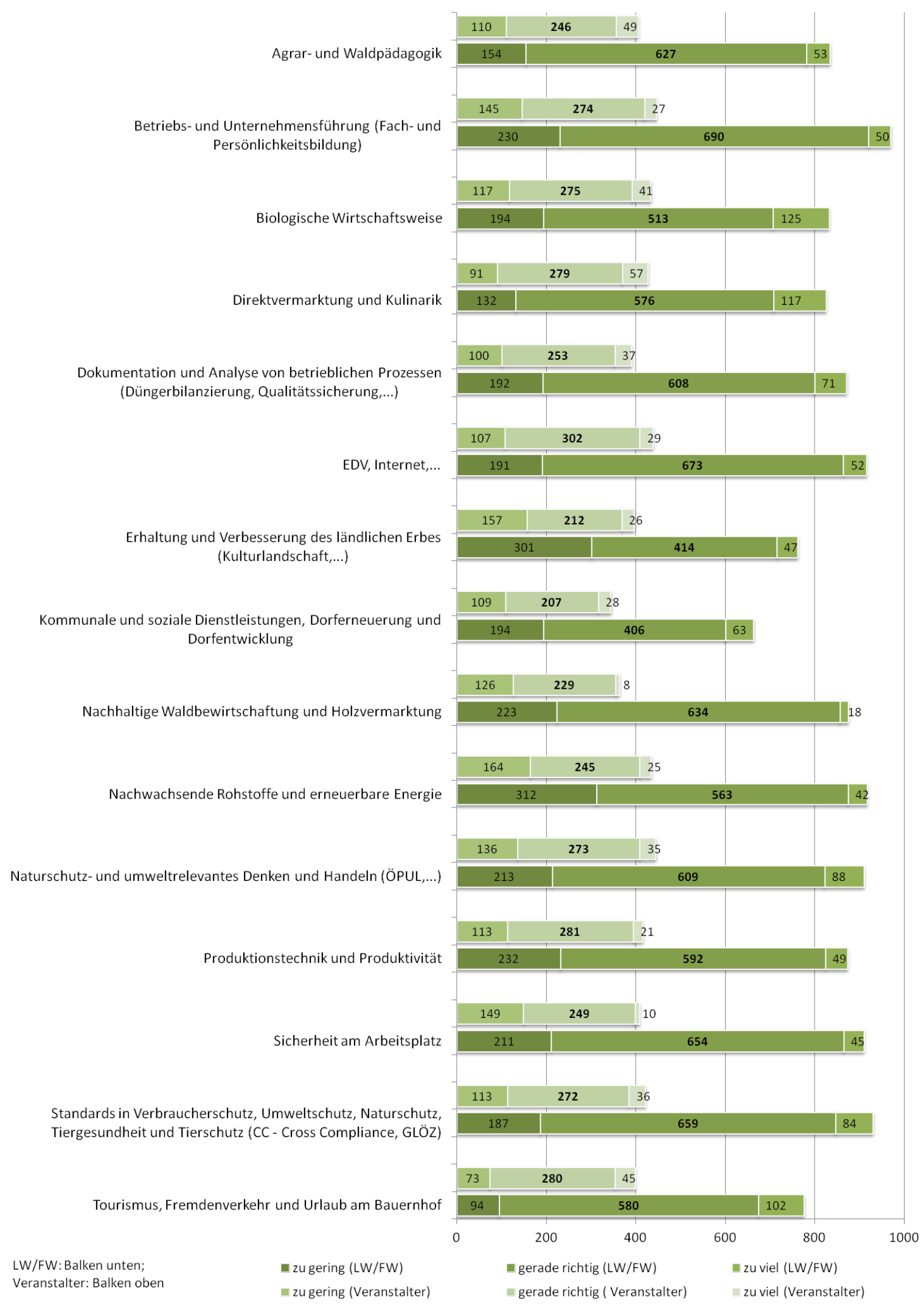


Abbildung 100b: Bewertung des Bildungsangebotes (in \%) - Vergleich Veranstalter / öffentliche Dienststellen vs. $L W / F W$

Wie sind Ihrer Einschätzung nach die folgenden Themen im Bildungsangebot der LE vertreten?

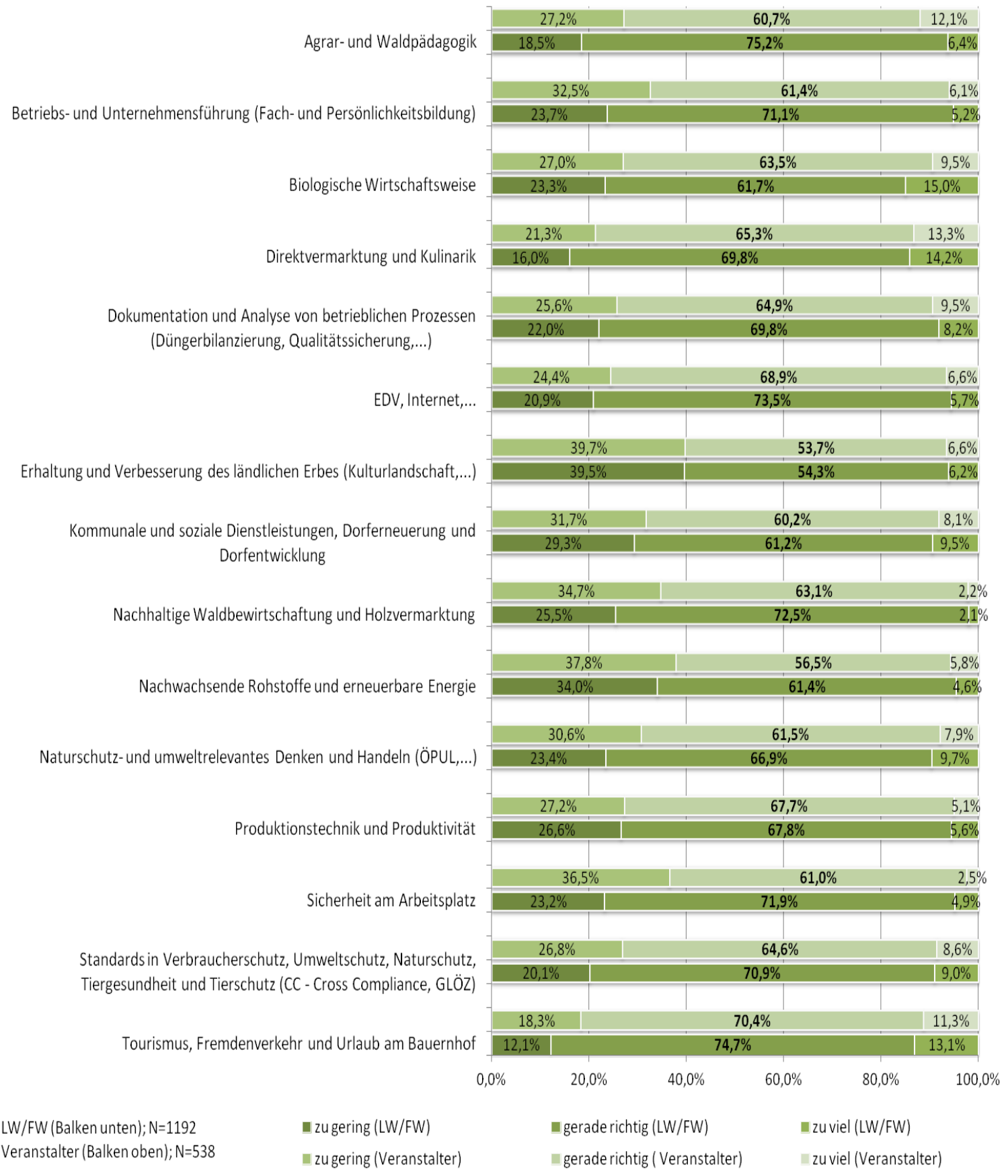


Aus Abbildung 101 gehen etwaige Schwerpunkte im Bildungsangebot der Veranstalter hervor. Manche Themen werden vorrangig von einer Teilgruppe der Bildungsveranstalter angeboten - z.B. "Produktionstechnik und Produktivität" von den LFIs, oder „Nachhaltige Waldbewirtschaftung und Holzvermarktung" durch die Forstlichen Ausbildungsstätten.

\section{Abbildung 101: Themen der Bildungsangebote - Veranstalter im Vergleich}

Zu welchen der folgenden Themen haben Sie selbst Bildungsangebote in der LE erstellt bzw. durchgeführt?

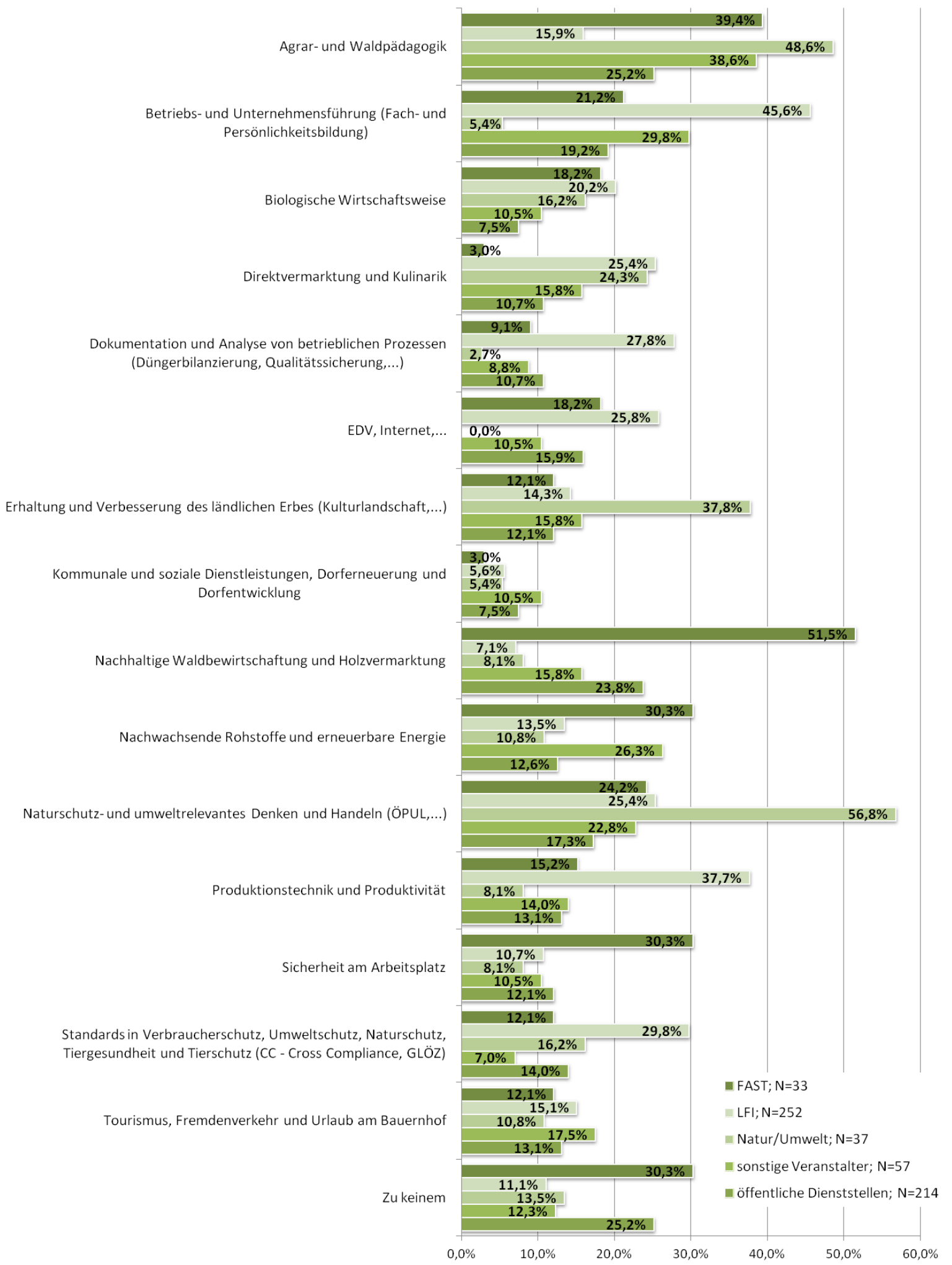


Die Auswertung in Abbildung 102 geht näher auf das Angebot seitens der Veranstalter und das Interesse bzw. die Nachfrage an bestimmten Themen bzw. Kursen und Veranstaltungen seitens der Land- bzw. ForstwirtInnen ein. Dabei fallen vor allem die Unterschiede bei den Themengebieten "Agrar- und Waldpädagogik", "Erhaltung und Verbesserung des ländlichen Erbes", "Nachhaltige Waldbewirtschaftung und Holzvermarktung" und "Naturschutz- und umweltrelevantes Denken und Handeln" auf.

\section{Abbildung 102: Angebot und Nachfrage an Bildungsangeboten}

Zu welchen der folgenden Themen haben Sie selbst Bildungsangebote in der LE erstellt bzw. durchgeführt? / Zu welchen der folgenden Themen haben Sie Bildungsangebote der LE besucht?

Agrar- und Waldpädagogik

Betriebs- und Unternehmensführung (Fach- und Persönlichkeitsbildung)

Biologische Wirtschaftsweise

Direktvermarktung und Kulinarik

Dokumentation und Analyse von betrieblichen Prozessen (Düngerbilanzierung, Qualitätssicherung,...)

EDV, Internet,...

Erhaltung und Verbesserung des ländlichen Erbes (Kulturlandschaft,...)

Kommunale und soziale Dienstleistungen, Dorferneuerung und Dorfentwicklung

Nachhaltige Waldbewirtschaftung und Holzvermarktung

Nachwachsende Rohstoffe und erneuerbare Energie

Naturschutz- und umweltrelevantes Denken und Handeln (ÖPUL,...)

Produktionstechnik und Produktivität

Sicherheit am Arbeitsplatz

Standards in Verbraucherschutz, Umweltschutz, Naturschutz, Tiergesundheit und Tierschutz (CC - Cross Compliance, GLÖZ)

Tourismus, Fremdenverkehr und Urlaub am Bauernhof
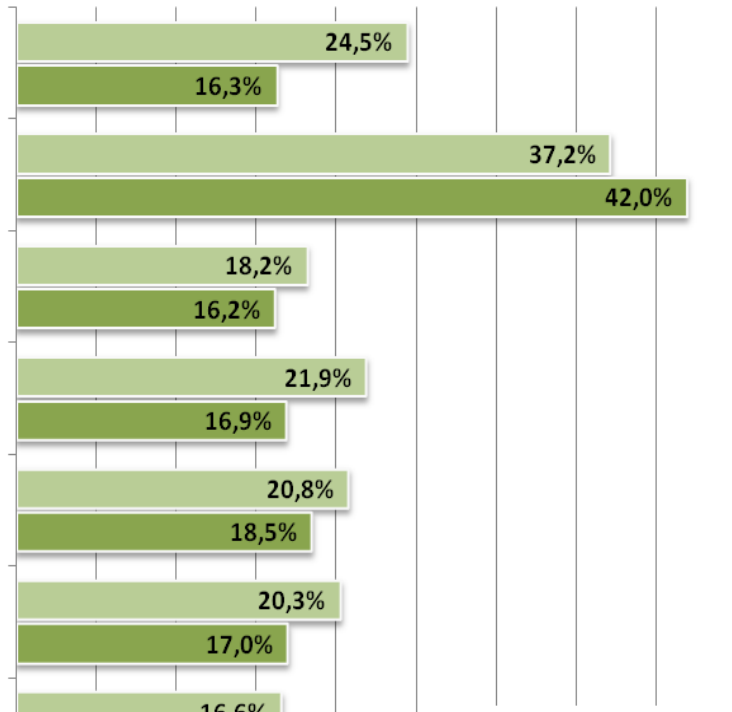

Veranstalter; $\mathrm{N}=379$

LW/FW; $\mathrm{N}=1086$

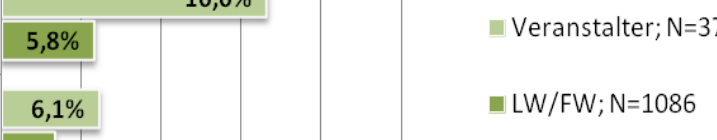

$2,4 \%$
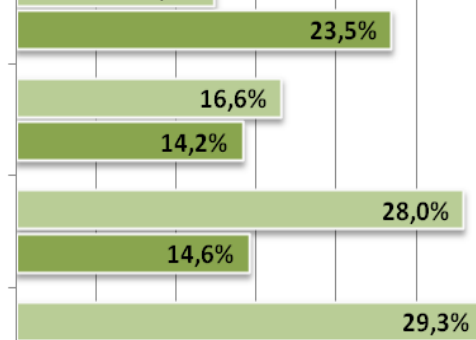

$23,0 \%$

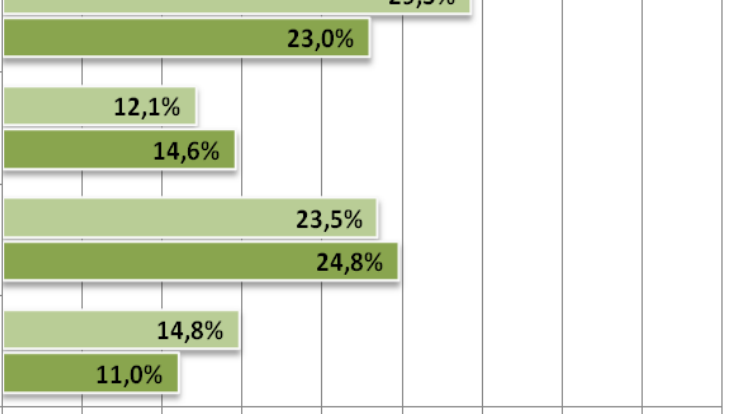

$1,0 \%$

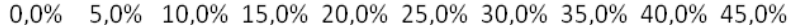


Unterschiedlich stark werden von den einzelnen Veranstaltern die verschiedenen Medien bei der Bewerbung der Bildungsveranstaltungen genutzt. Sehr beliebt bei allen Teilgruppen der Veranstalter ist der Einsatz von Broschüren, Foldern und Katalogen sowie des Internets. Von den LFIs werden auch sehr stark die Kammerzeitungen genutzt.

Abbildung 103: Bewerbung der Bildungsangebote - Veranstalter im Vergleich

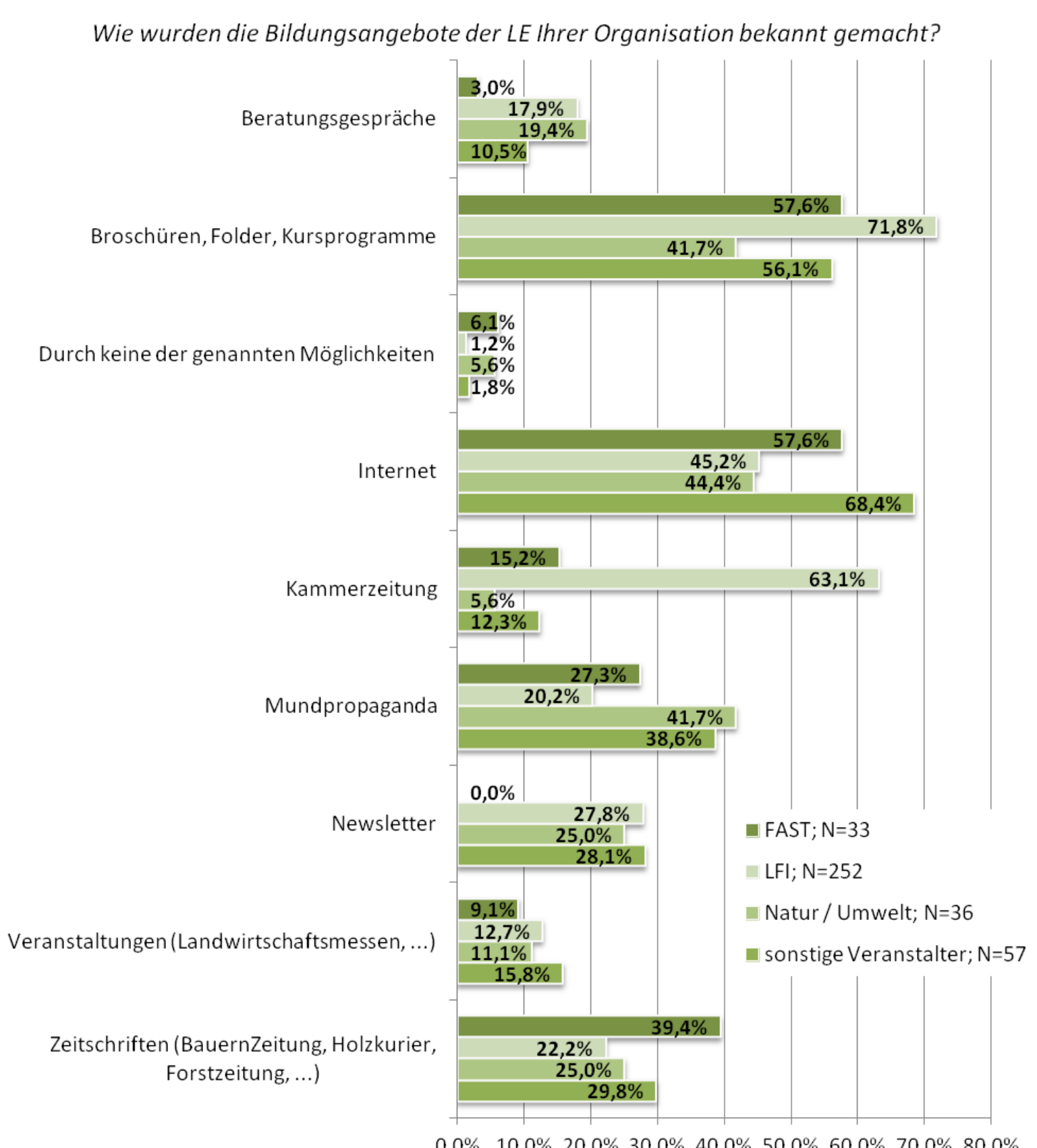

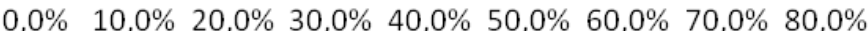


Beim Vergleich der Antworten der Veranstalter zur vorherigen, in Abbildung 103 dargestellten Frage, und jenen der Land- bzw. ForstwirtInnen zeigt sich, dass Werbeaufwand seitens der Veranstalter und Wahrnehmung der Kurse über das jeweilige Medium seitens der Land- bzw. ForstwirtInnen sich nicht immer decken. Die Land- bzw. ForstwirtInnen beziehen Informationen beispielsweise viel stärker aus Zeitschriften wie Bauernzeitung oder Holzkurier, als diese von den Veranstaltern genutzt werden (siehe Abbildung 104).

Abbildung 104: Bewerbung der Bildungsangebote und Wahrnehmung seitens der LW /FW

Wie wurden die Bildungsangebote der LE Ihrer Organisation bekannt gemacht? / Wie wurden Sie auf die Bildungsangebote aufmerksam?

Beratungsgespräch

Broschüren, Folder, Kursprogramme
Internet
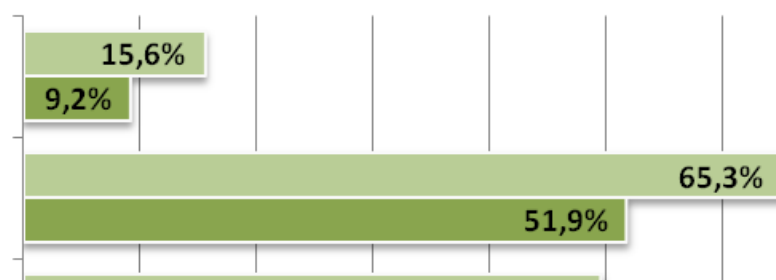

$65,3 \%$

Kammerzeitung

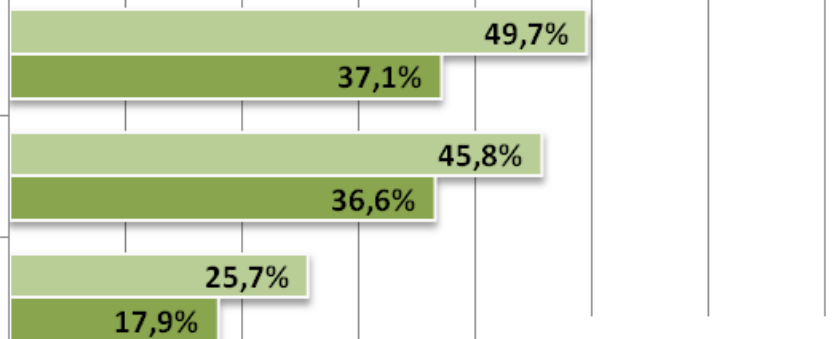

Newsletter

Persönliche Empfehlung

Veranstaltungen (Landwirtschaftsmessen, ...)

Zeitschriften (BauernZeitung, Holzkurier, Forstzeitung, ...)

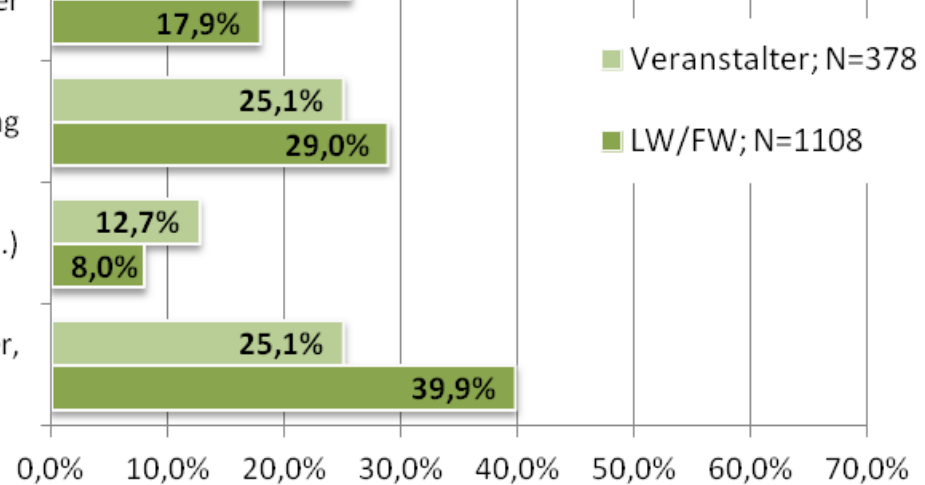


Sehr unterschiedlich stellen sich die Auswertungen der einzelnen Teilgruppen der Veranstalter zur Frage dar, wie sich die Situation in der Land- und Forstwirtschaft seit 2007 verändert hat.

\section{Abbildung 105: Einschätzung der Veränderung seit 2007 - Veranstalter im Vergleich}

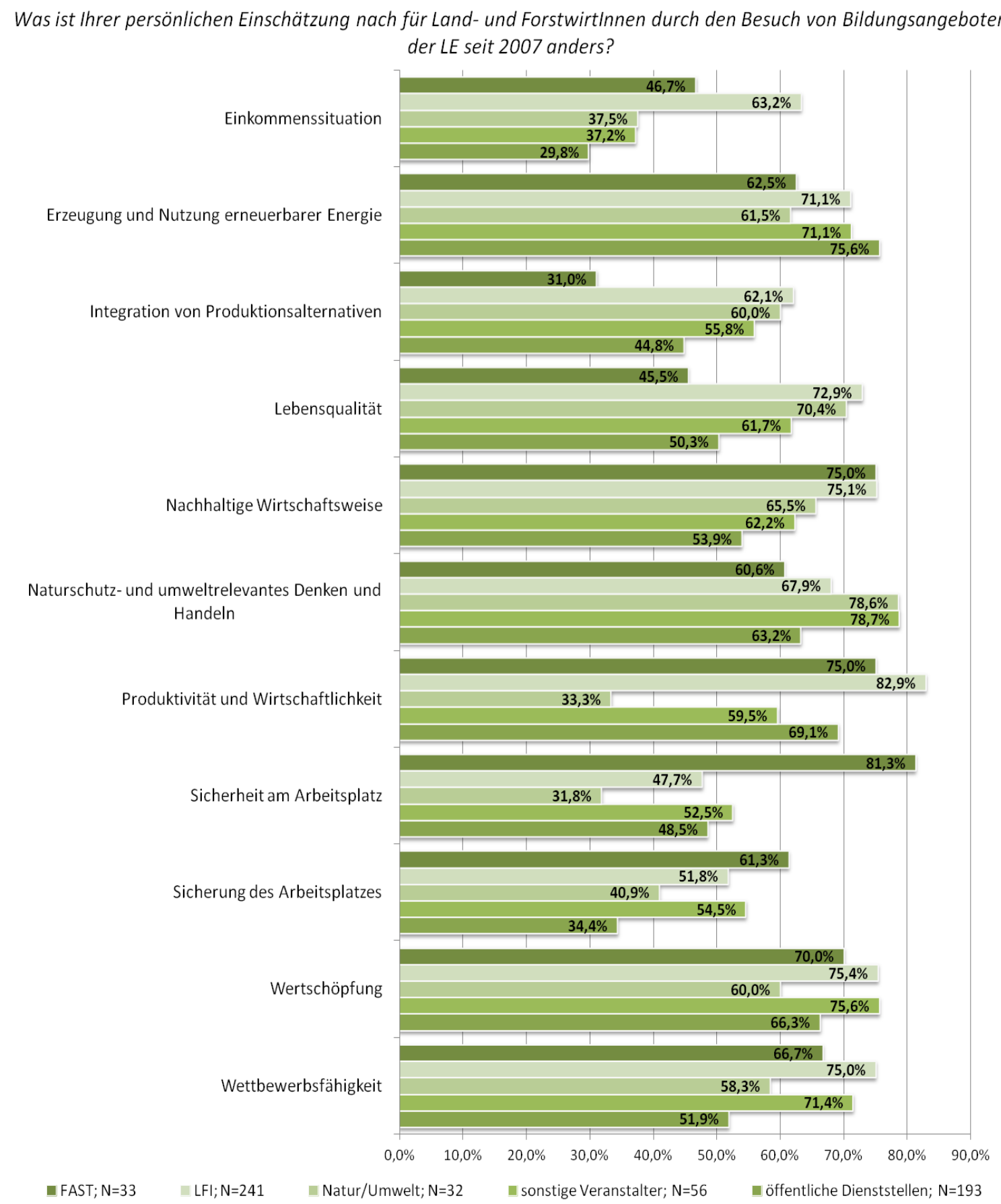

Werden die Einschätzungen von Veranstaltern jenen der Land- bzw. ForstwirtInnen, die Bildungsangebote in Anspruch genommen haben, gegenüber gestellt fällt auf, dass die Einschätzungen der Veranstalter deutlich positiver sind, als die Einschätzung bzw. Wahrnehmung der Land- bzw. ForstwirtInnen selbst. Am stärksten zeigt sich der Unterschied beim Vergleich der Angaben zur Veränderung der Einkommenssituation. 
Abbildung 106: Einschätzung der Veränderung seit 2007 - Vergleich Veranstalter vs. LW / FW

Was ist Ihrer persönlichen Einschätzung nach für Land- und ForstwirtInnen durch den Besuch von Bildungsangeboten der LE seit 2007 anders? / Was ist für Sie auf Ihrem land- und forstwirtschaftlichen Betrieb bzw. im Ländlichen Raum seit 2007 anders?

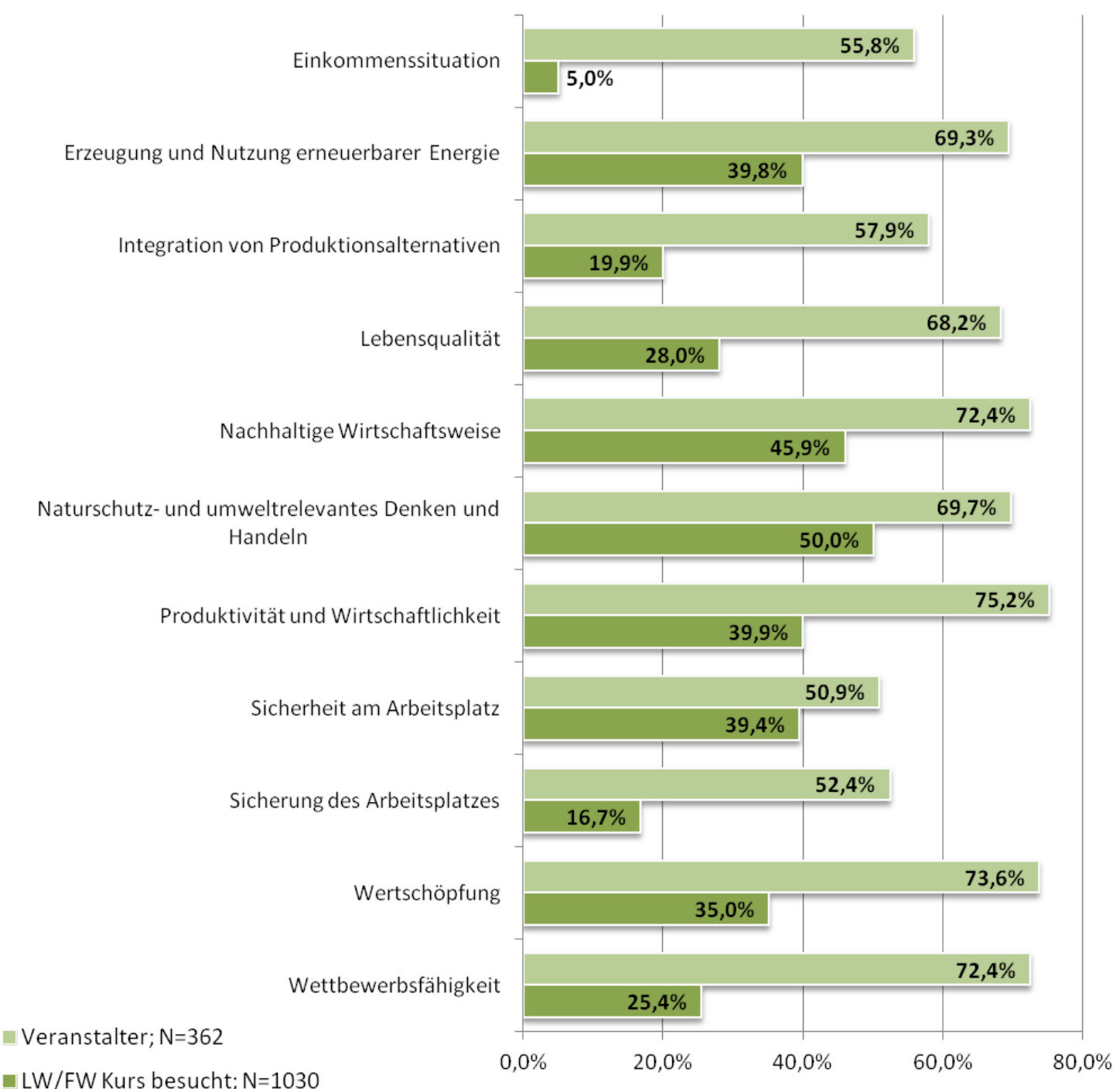




\subsubsection{TeilnehmerInnen an Kursen der LE aus anderen Branchen}

Der Fragebogen erreichte auch Personen, die zwar Bildungsveranstaltungen der Ländlichen Entwicklung besuchten, jedoch in anderen Branchen als der Land- und Forstwirtschaft ihren beruflichen Tätigkeiten nachgehen und somit nur teilweise zu den Zielgruppen gemäß Bildungsmaßnahme M 331 und jedenfalls nicht zur Zielgruppe gemäß Bildungsmaßnahme M 111 gehören. In diesem Kapitel wird auf diese Personengruppe näher eingegangen bzw. werden auch Vergleiche mit den Aussagen der Land- bzw. ForstwirtInnen hergestellt.

Die schulische Ausbildung unterscheidet sich stark von der der Land- bzw. ForstwirtInnen. Die meisten der TeilnehmerInnen aus nicht-agrarischen Berufen haben einen Hochschulabschluss.

Abbildung 107: Schulbildung - Vergleich nach Branche

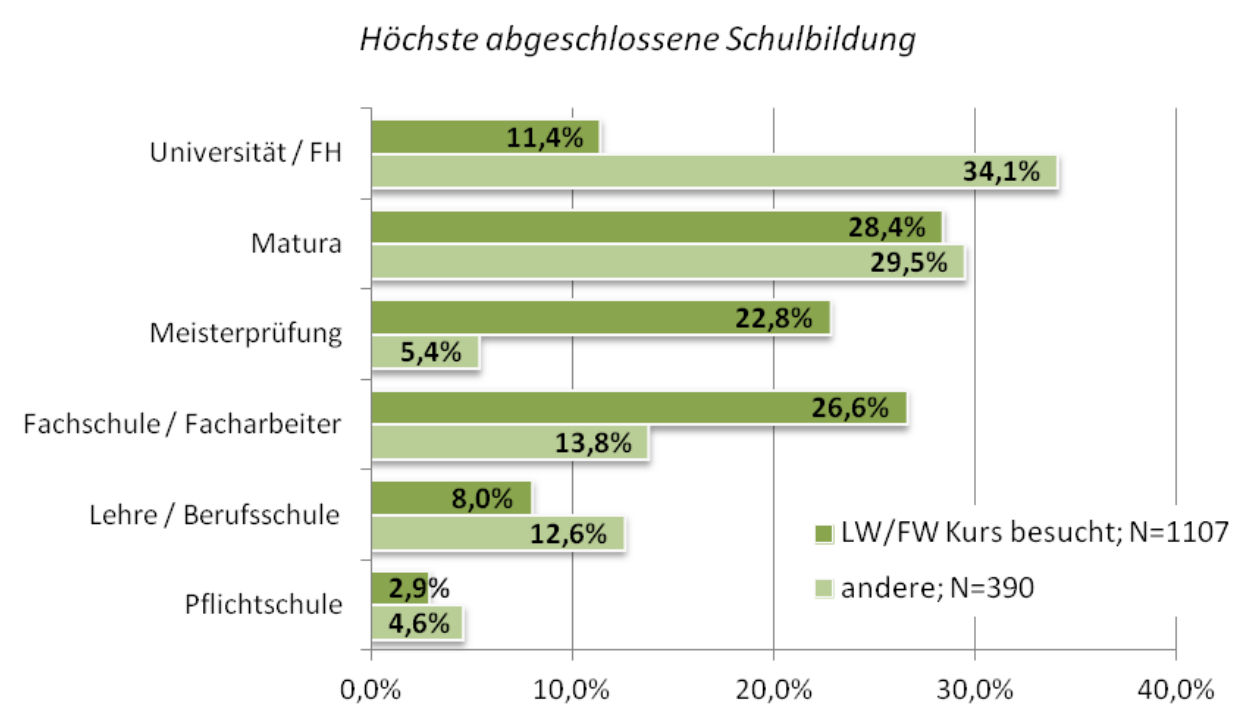

Während die Land-bzw. ForstwirtInnen als Bildungsanbieter Großteils die Ländlichen Fortbildungsinstitute in den Bundesländern wählten, war der Großteil der befragten Personen aus anderen Branchen an Aus- bzw. Weiterbildungen der Forstlichen Ausbildungsstätten interessiert. 
Abbildung 108: Gewählte Bildungsanbieter - Vergleich nach Branche

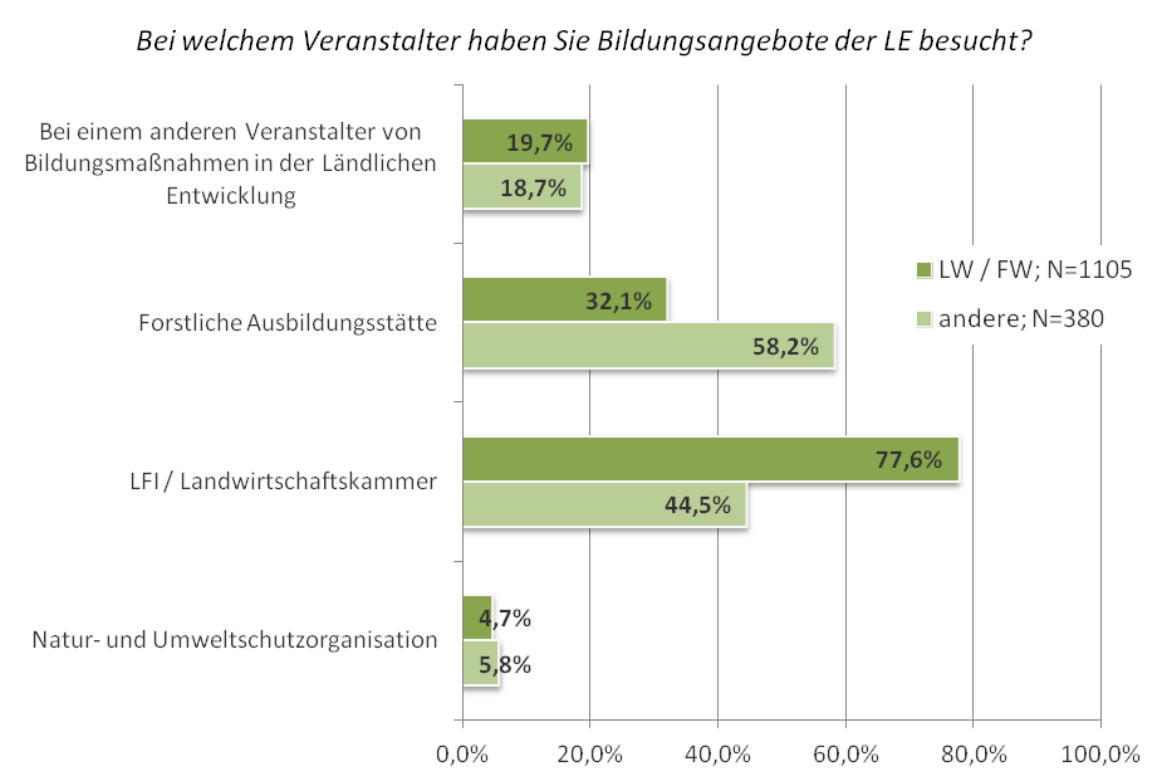

Deutlich stärker als bei den Land-bzw. ForstwirtInnen kamen die Personen aus nicht-land- bzw. forstwirtschaftlichen Bereichen durch persönliche Empfehlungen zu den Kursen der LE. Die Kammerzeitung sowie andere agrarische Zeitschriften werden hingegen eher weniger als Informationsquelle zu den Kursangeboten gesehen bzw. sind in dieser Gruppe weniger verbreitet.

Abbildung 109: Informationsquellen zu den Bildungsangeboten - Vergleich nach Branche

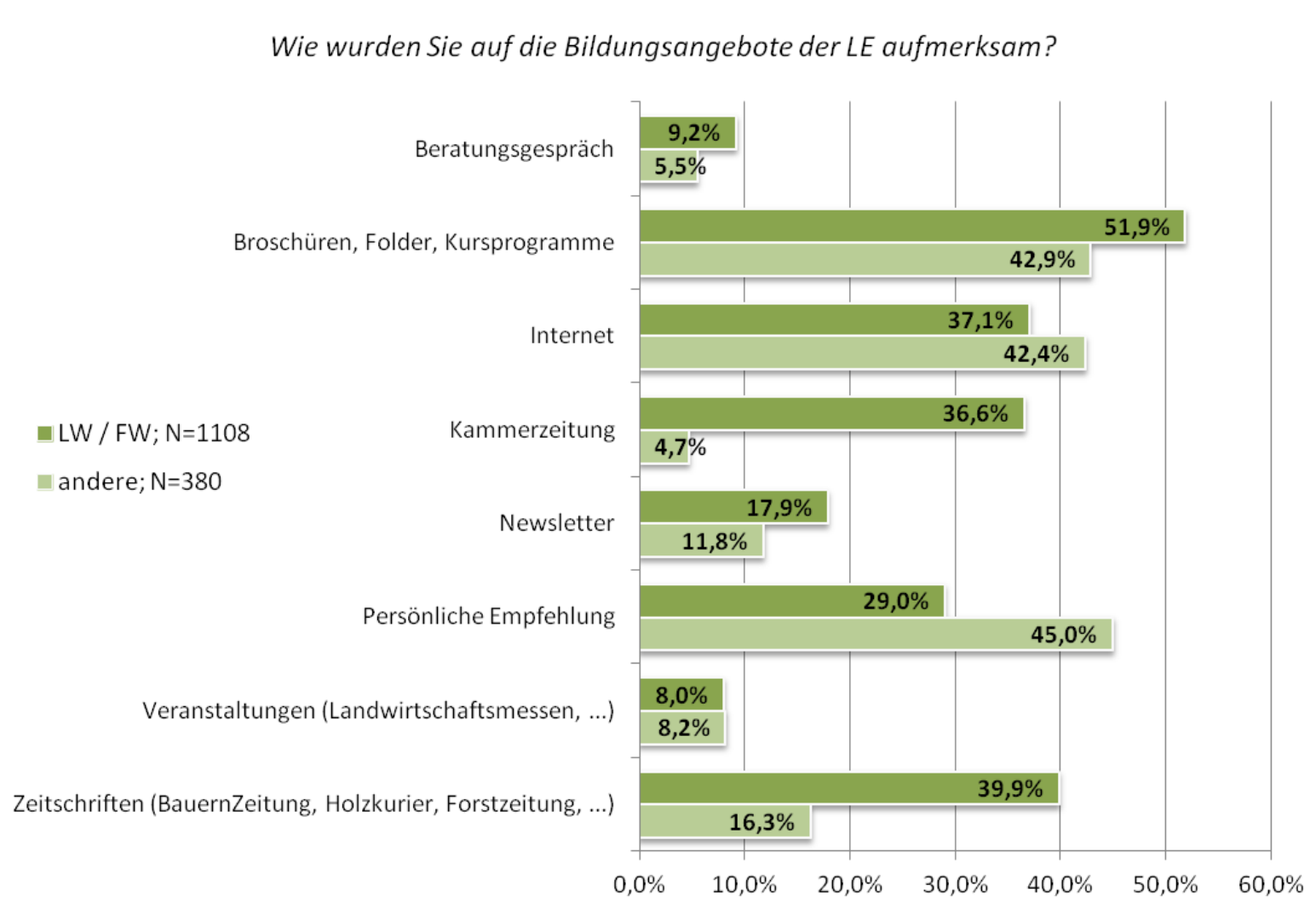


Die TeilnehmerInnen aus anderen Branchen sehen vor allem bei den Themenbereichen „Biologische Wirtschaftsweise", "Standards in Verbraucherschutz, Umweltschutz, Naturschutz etc." und "Naturschutz- und umweltrelevantes Denken und Handeln" mehr noch als die Land-bzw. ForstwirtInnen ein zu geringes Angebot.

\section{Abbildung 110a: Bewertung des Bildungsangebotes (absolut) - Vergleich nach Branche}

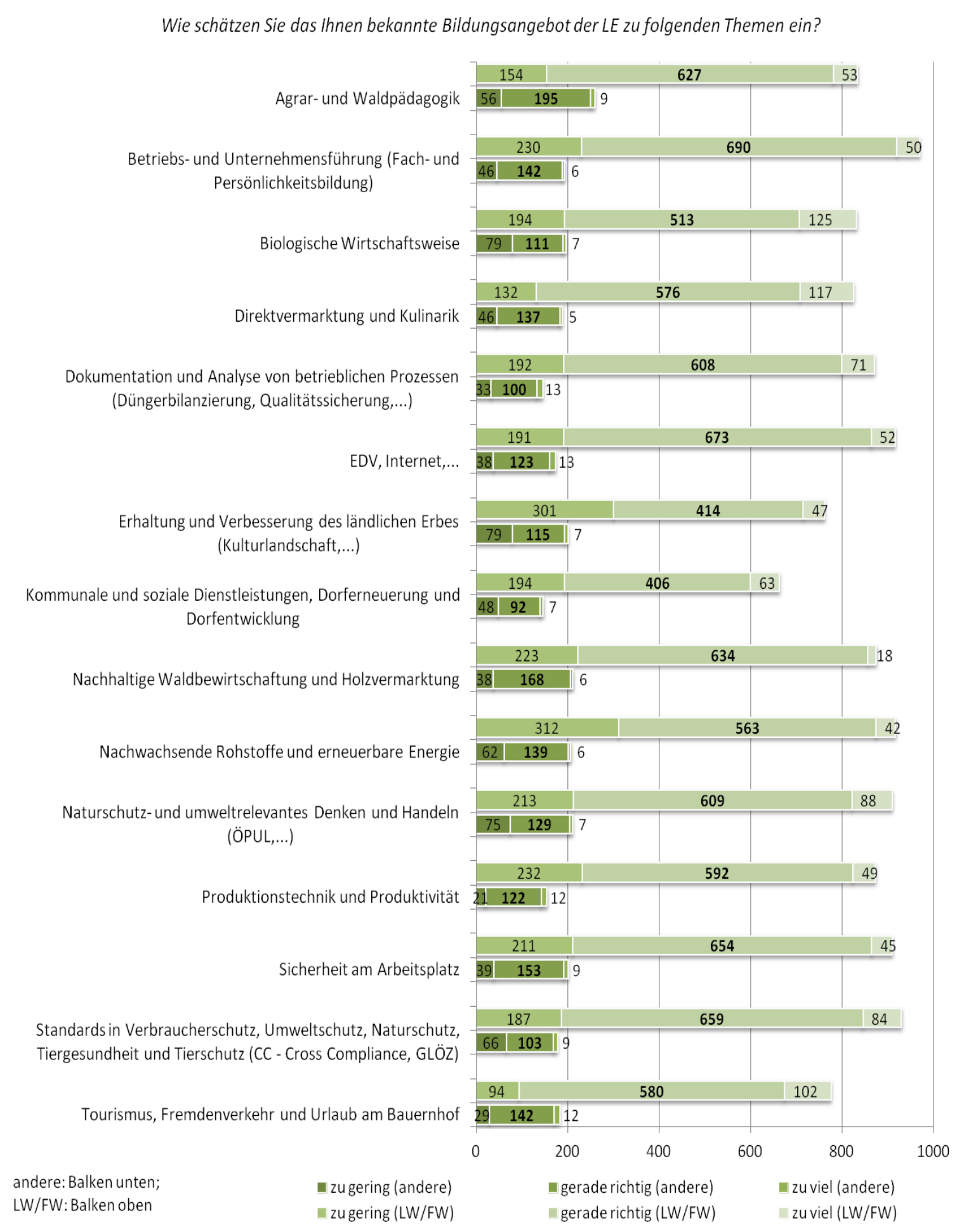




\section{Abbildung 110b: Bewertung des Bildungsangebotes (in \%) - Vergleich nach Branche}

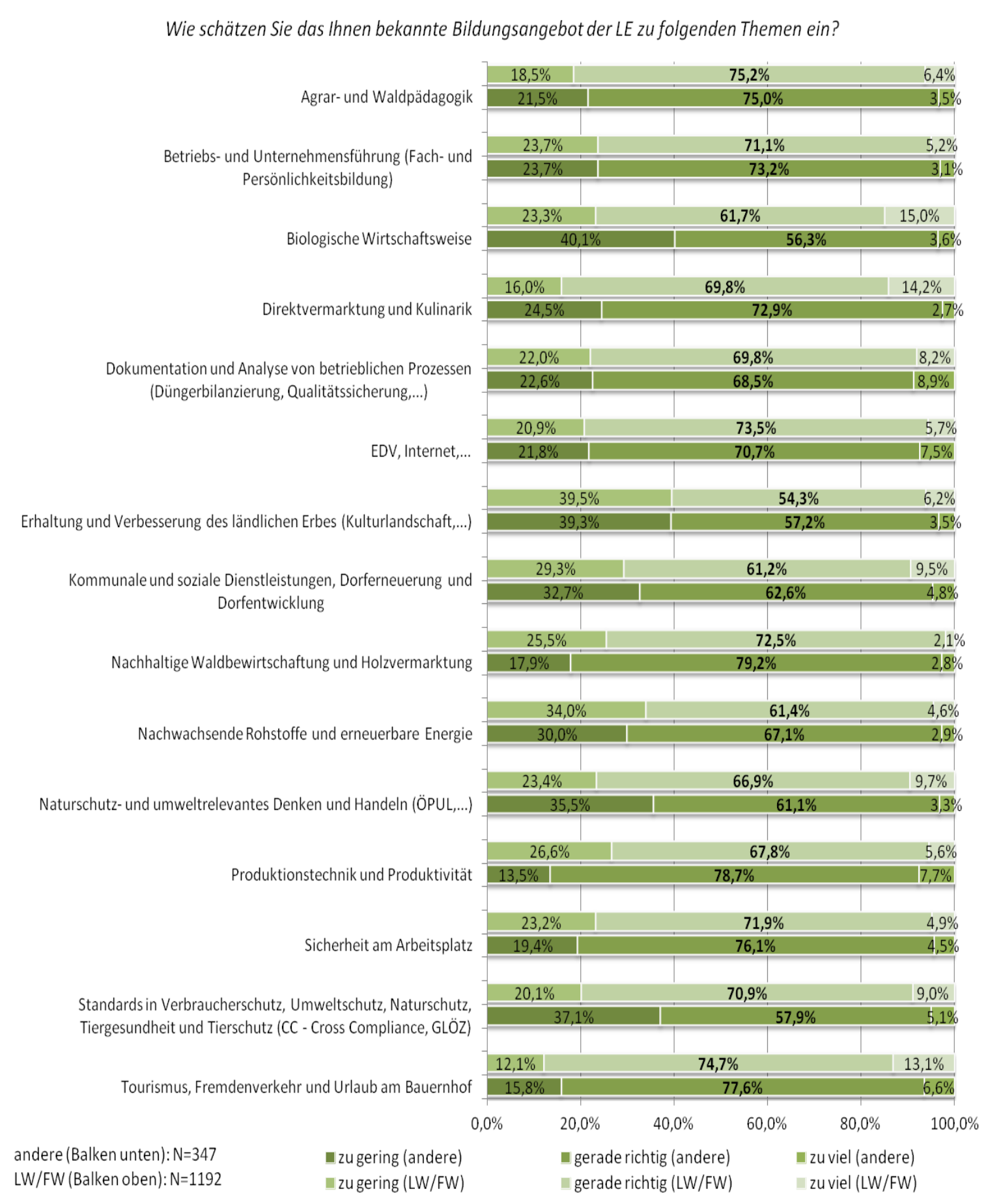


Sehr wichtig sind für die Kursteilnehmer aus anderen Branchen, so wie auch bei den Land- bzw. ForstwirtInnen, das jeweilige Thema der Bildungsangebote, der erwartete Nutzen, und Qualifikation und Kompetenz der Trainer. Die Kosten der Bildungsveranstaltungen und eine eventuelle persönliche Empfehlung zu einem Kurs nehmen für die Nicht-Land- bzw. ForstwirtInnen einen deutlich höheren Stellenwert bei der Entscheidung über eine mögliche Kursteilnahme ein.

\section{Abbildung 111: Auswahlkriterien - Vergleich nach Branche}

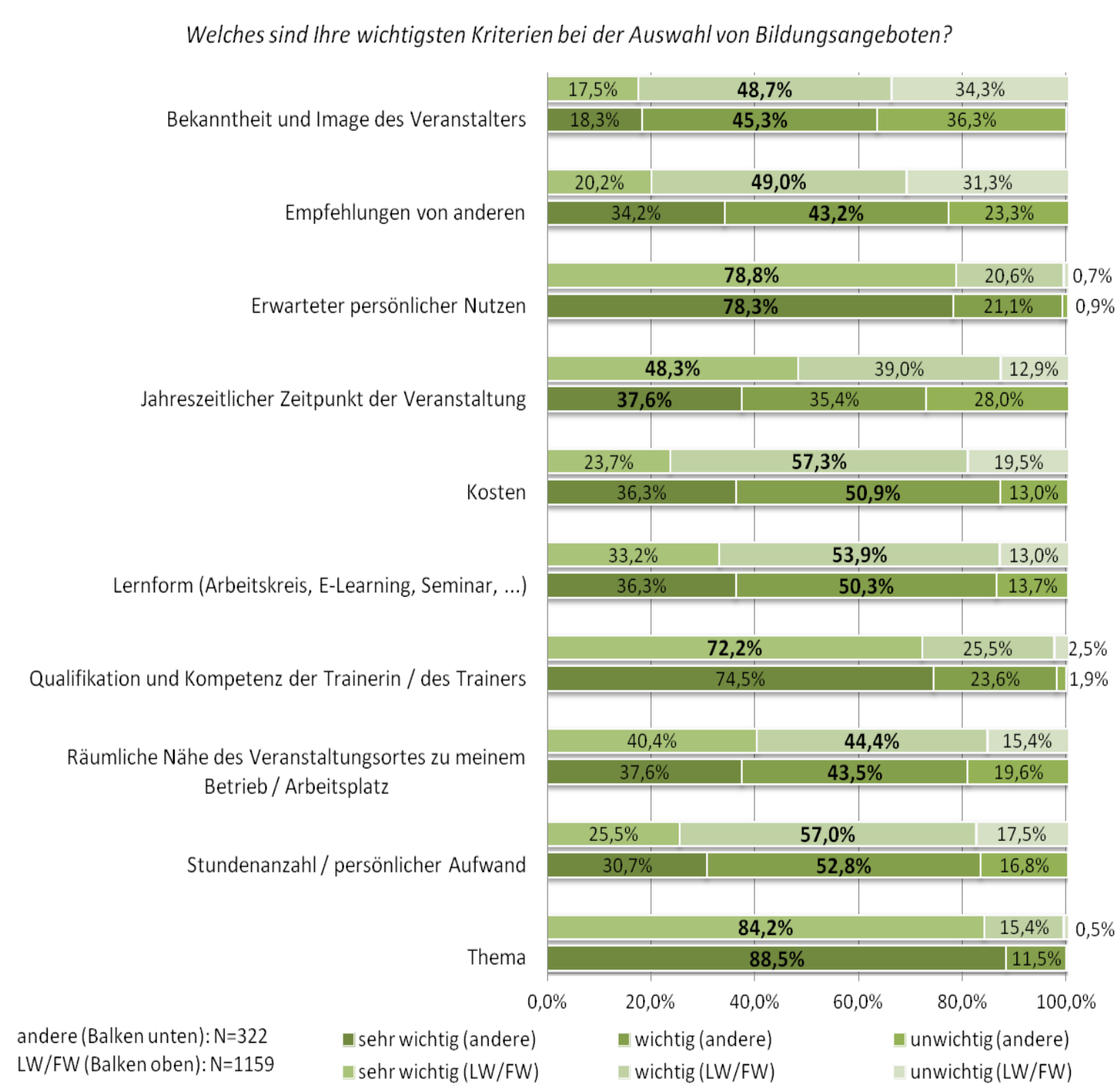


Sehr unterschiedlich ist auch das Interesse bzgl. der verschiedenen Themen der Kurse. Seitens der Personen aus anderen Branchen besteht starkes Interesse an Angeboten zu Agrar- und Waldpädagogik. Kurse zu Prozessen bzw. Aspekten alltäglicher Tätigkeiten in Land- und Forstwirtschaft wurden vergleichsweise deutlich weniger nachgefragt. Das hohe Interesse der Personen aus anderen Branchen an Kursen zum Thema "Nachhaltige Waldbewirtschaftung und Holzvermarktung" weist auf die vielen hoffernen / urbanen / neuen Waldbesitzer in Österreich hin.

Abbildung 112: Besuchte Kurse - Vergleich nach Branche

Zu welchen der folgenden Themen haben Sie Bildungsangebote der LE besucht?

Agrar- und Waldpädagogik

Betriebs- und Unternehmensführung

(Fach- und Persönlichkeitsbildung)

Biologische Wirtschaftsweise

Direktvermarktung und Kulinarik

Dokumentation und Analyse von betrieblichen Prozessen...

EDV, Internet,...

Erhaltung und Verbesserung des ländlichen Erbes (Kulturlandschaft,...) Kommunale und soziale Dienstleistungen, Dorferneuerung und Dorfentwicklung

Nachhaltige Waldbewirtschaftung und Holzvermarktung

Nachwachsende Rohstoffe und erneuerbare Energie

Naturschutz- und umweltrelevantes Denken und Handeln (ÖPUL,...)

Produktionstechnik und Produktivität

Sicherheit am Arbeitsplatz

Standards in Verbraucherschutz, Umweltschutz, Naturschutz,...

Tourismus, Fremdenverkehr und Urlaub am Bauernhof
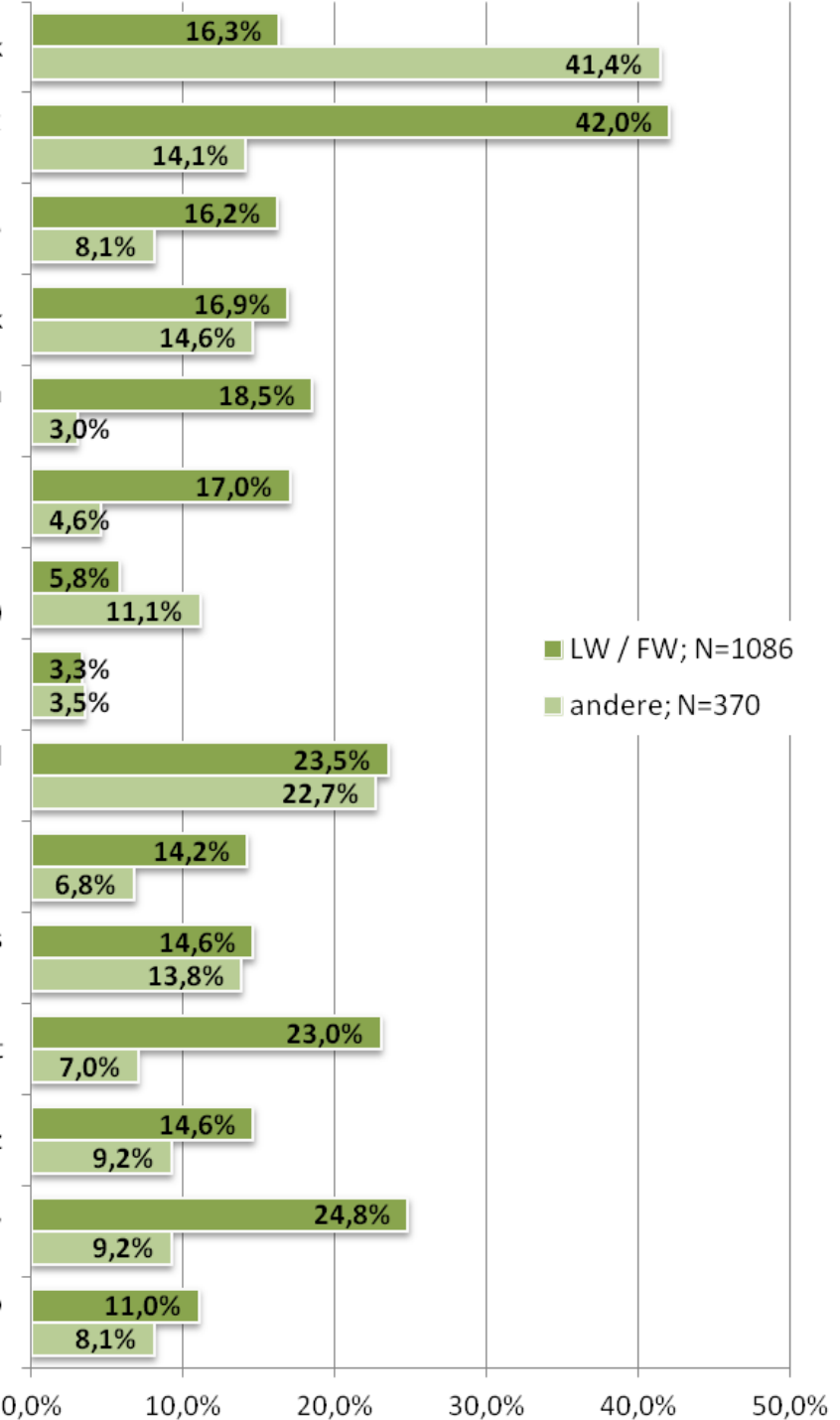
Die zur Bewertung gestellten Lernformen wurden in beiden Vergleichsgruppen in etwa gleich eingestuft. Auch bei Nicht-Land- bzw. ForstwirtInnen sind "Seminare und Kurse", "Erfahrungsaustausch und Arbeitskreise“ und „Betriebsbesuche / Exkursionen" die drei beliebtesten Lernformen.

Abbildung 113: Lernformen - Vergleich nach Branche

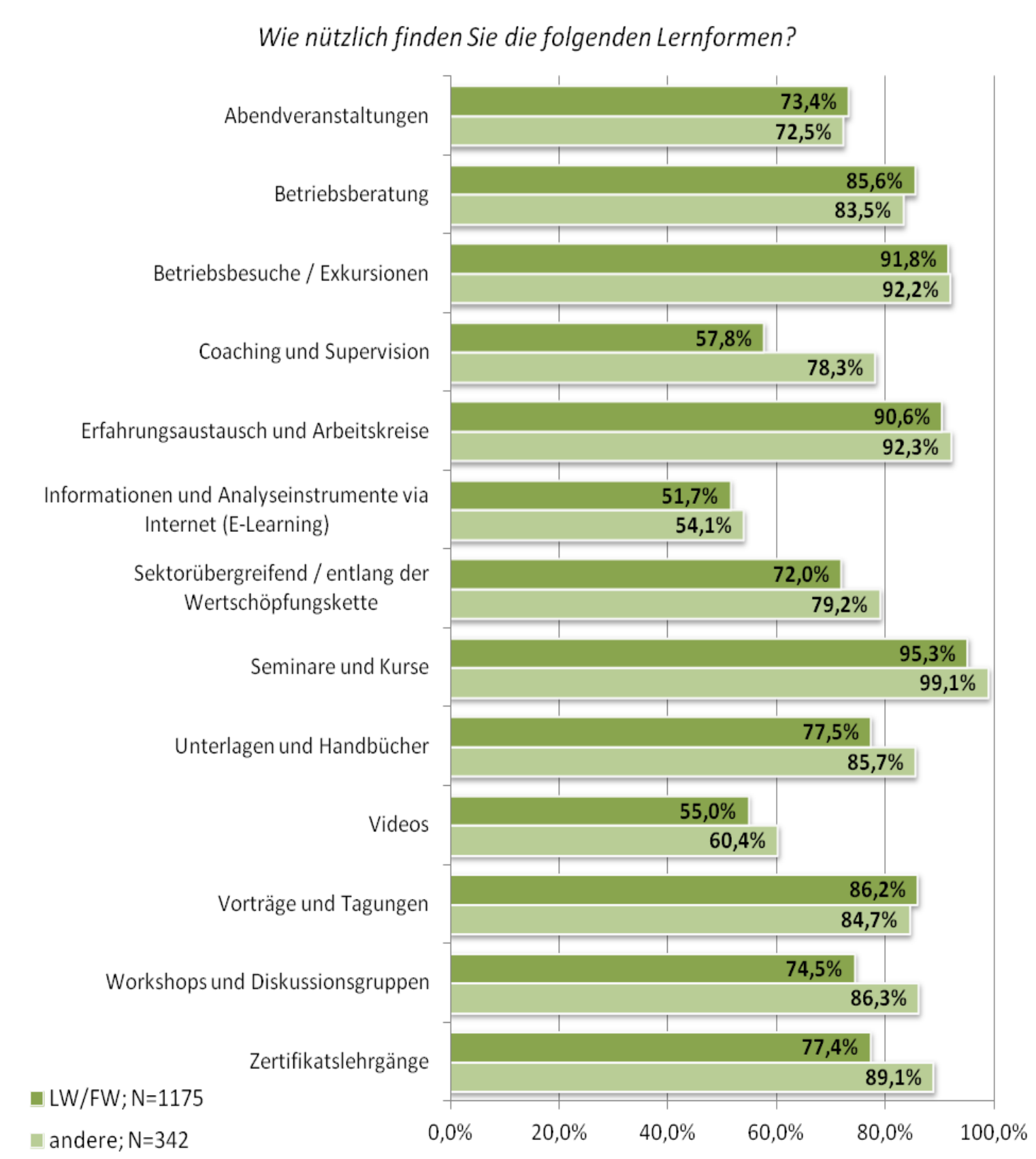


Personen aus nicht-agrarischen Berufen empfinden den Nutzen der LE-Bildungsangebote noch etwas positiver, als die Vergleichsgruppe der Land- bzw. ForstwirtInnen.

\section{Abbildung 114: Empfundener Nutzen der Bildungsangebote - Vergleich nach Branche}

Wie nützlich fanden Sie die Bildungsangebote der LE für Ihre berufliche Weiterentwicklung insgesamt?

Sehr nützlich - Hat mich entscheidend weiter gebracht

Nützlich - Ich habe neue / weiterführende Impulse für meine berufliche Tätigkeit erhalten.

Wenig nützlich - Geringe Verbesserung zu früher feststellbar.

Kein Effekt - Es ist für mich kein Unterschied zu früher feststellbar.
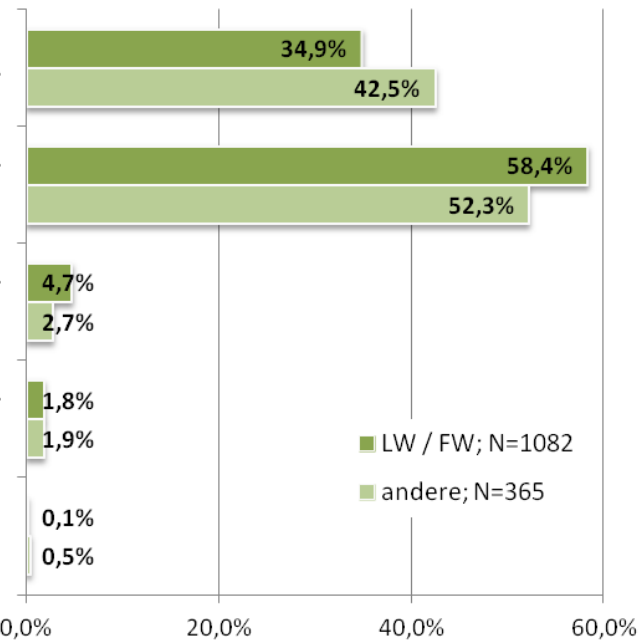

Die Einschätzungen der Nicht-Land- und ForstwirtInnen bezüglich der Veränderungen im Ländlichen Raum liegen näher bei den Einschätzungen der Land- bzw. ForstwirtInnen als jene der Veranstalter (vgl. Abbildung 106). Teilweise bewerteten die befragten Personen aus anderen Branchen die Situation pessimistischer als die Land- bzw. ForstwirtInnen.

Abbildung 115: Veränderungen seit 2007 - Vergleich nach Branche

Was ist für Sie auf Ihrem land- und forstwirtschaftlichen Betrieb bzw. im Ländlichen Raum seit 2007 anders?

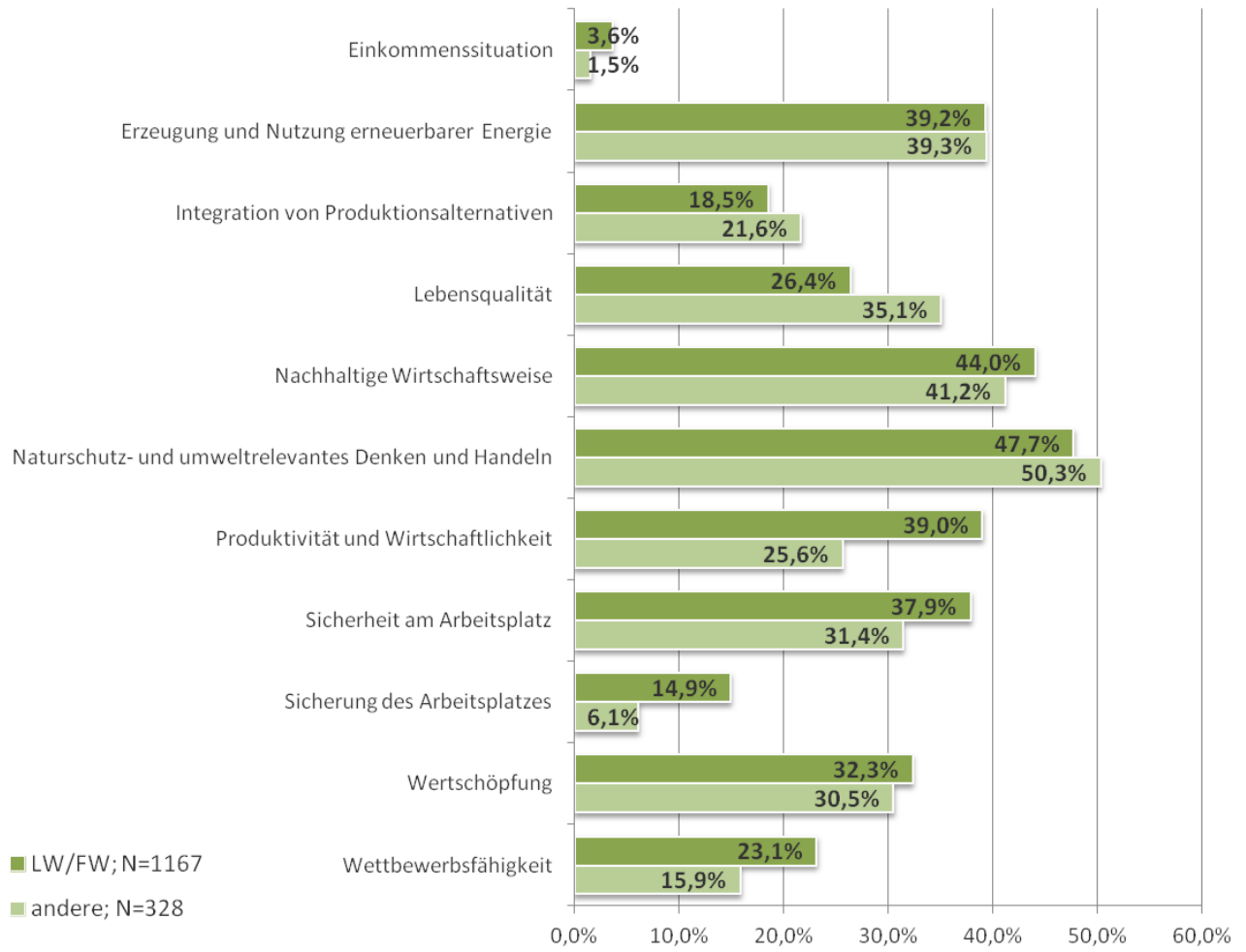




\section{VERBESSERUNGSPOTENTIALE}

Dass die Bildungsmaßnahmen M 111, M 331, Agrar-Umwelt und Naturschutz sowie Forstbildungsmaßnahmen seit 2007 Nutzen und Wirkung erbracht haben, geht klar aus Kapitel 4 hervor. Dabei ergibt sich ein komplexes, differenziertes und durchaus positives Bild. Somit könnte auch der Standpunkt vertreten werden, dass sich die bisherigen Bildungsmaßnahmen bewährt haben und in dieser Form beibehalten und weitergeführt werden sollten. Mehrere Gründe sprechen aber dagegen:

- Auch wenn Nutzen und Wirkung ersichtlich sind, bedeutet dies nicht, dass die Maßnahmen Pareto-effizient sind. Pareto-Effizienz bezeichnet in der Ökonomie einen Zustand, in dem es nicht möglich ist, eine Eigenschaft zu verbessern, ohne zugleich eine andere verschlechtern zu müssen. Auf Grund der permanenten gesellschaftlichen Veränderung ist Pareto-Effizienz auch nicht ein Zustand, der, wenn er einmal erreicht ist, aufrecht bleibt. Daher macht es Sinn, immer wieder nach Pareto-effizienteren Fördermaßnahmen zu suchen.

- Das Förderprogramm LE 14-20 setzt mit "Wissenstransfer und Innovation" deutlich neue Schwerpunkte gegenüber LE 07-13. Während Weiterbildung die "Fortsetzung oder Wiederaufnahme organisierten Lernens nach Abschluss einer unterschiedlich ausgedehnten ersten Bildungsphase" ist, bezeichnet Wissenstransfer den Austausch und die Beziehungen zwischen Wissenschaft und Praxis. Hierbei kann sowohl im engeren Sinne eine Kooperation zwischen Wissenschaft und land- bzw. forstwirtschaftlichen Betrieben, als auch im weiteren Sinne jegliche Form der Wissenschaftskommunikation in die Praxis gemeint sein. Innovation schließlich ist eine neue Leistung, ein neues Produkt, welche neuen Nutzen stiftet, neuen Anforderungen oder unausgesprochenen Bedürfnissen gerecht wird und daher von Märkten oder von der Gesellschaft angenommen wird und sich verbreitet. Dieser geänderte Fokus des Programms LE hat zur Folge, dass die Bildungsmaßnahmen um Wissenstransfer- und InnovationsMaßnahmen erweitert werden müssen.

- Bekannt ist, dass die Evolution Vorbild für (land-) wirtschaftliche Entwicklung vor allem bei Innovationsfähigkeit als auch bei Resilienz, d.h. Widerstandsfähigkeit, ist. Daraus hat sich auch als neuer Wissenschaftszweig die „Evolutionsökonomik" herauskristallisiert. Basierend darauf, sowie auf Erkenntnissen der Inselbiogeographie ergeben sich für die wirkungsvolle Förderung von Wissenstransfer und Innovation neue Ansätze.

In diesem Sinne zeigen die im Folgenden dargestellten Verbesserungspotenziale Möglichkeiten auf, um die, in der neuen Förderperiode im Zentrum der Priorität 1 stehenden Schwerpunkte "Wissenstransfer und Innovation" zielgerichtet und an die Bedürfnisse der Land- bzw. Forstwirtschaft angepasst zu gestalten und anzubieten.

\subsection{Prioritäten, Ziele und Schwerpunktsetzungen für LE 14-20 Priorität I}

Die verschiedenen, in diesem Kapitel ausführlich betrachteten Verbesserungspotentiale wurden für die folgenden Bereiche identifiziert: Qualität der Maßnahmengestaltung; Themen der Bildungsangebote; Lernformen der Bildungsangebote. Zusätzlich werden für zukünftige Monitoringsund Evaluierungsprozesse Wirkungsindikatoren spezifiziert und in diesem Zusammenhang eine Strukturierung der zu sammelnden Daten vorgeschlagen.

\subsubsection{Qualität der Maßnahmengestaltung}

Aus der Online-Umfrage sowie den Dialog-Interviews lassen sich eine Reihe von Schlussfolgerungen bezüglich des Nutzen und der Schwächen der bisherigen Maßnahmenumsetzung ziehen. 


\subsubsection{Doppelgleisigkeit}

Doppelgleisigkeiten sind bei der Förderung der Vorbereitung, Durchführung und Nachbereitung von Berufsbildungs- und Informationsmaßnahmen vielfach gegeben, weil die Förderung auch darauf abzielt, dass die Maßnahmen zu den Land- und ForstwirtInnen kommen und nicht umgekehrt. Deshalb gibt es in verschiedenen Regionen viele, einander ähnelnde Berufsbildungs- und Informationsmaßnahmen. Vorteil dieser Doppelgleisigkeit ist, dass sie die Reisezeiten für die TeilnehmerInnen reduziert. Nachteil dieser Förderungsstrategie ist, dass ähnliche Berufsbildungs- und Informationsmaßnahmen in benachbarten Regionen einander konkurrenzieren. Dies führt mitunter dazu, dass keine der doppelgleisig angebotenen Veranstaltungen mangels genügender Anzahl TeilnehmerInnen stattfindet.

\subsubsection{Zielerreichung}

Wie bereits in Kapitel 4 ausführlich dargelegt, gibt es gemäß den Einschätzungen der Land- bzw. ForstwirtInnen einen klaren Zusammenhang zwischen den Berufsbildungs- und Informationsmaßnahmen und den angestrebten Wirkungs-Zielen.

\subsubsection{Verhältnismäßigkeit zwischen Kosten und Wirkungen}

Da die Wirkungen nicht-monetäre Größen sind - siehe z.B. Abbildung 24 - ist ein Vergleich zwischen Kosten und Wirkungen seriöser weise nicht möglich. Allerdings können aus Abbildung 25 das BMLFUW und die Europäische Kommission selbst die Verhältnismäßigkeit zwischen den Kosten gemäß Abbildung 2 und Abbildung 4 und den mit dieser Studie nunmehr erhobenen Wirkungen einschätzen. Was indes durch die Dialog-Interviews klar wurde ist, dass die Kosten der Förderungsabwicklung aus Sicht der geförderten Veranstalter in Relation zum Förderungsbetrag hoch sind und insbesondere auch höher als in der vorangehenden Periode der LE. Da der Abrechnungsaufwand bei kleineren Förderbeträgen nicht entsprechend niedriger ist als bei großen Förderbeträgen, sind bei kleinen Förderbeträgen, insbesondere auch aber nicht nur bei TeilnehmerlnnenFörderungen die Kosten der Förderungsabwicklung besonders hoch. Eine Vereinfachung der Abrechnungsformalitäten mit dem Ziel, die Kosten der Förderungsabwicklung deutlich zu senken, wird daher empfohlen. Aussagen hierzu aus den Dialog-Interviews:

"Größte Hürde sind Haftungsfragen und Vorfinanzierung - ca. ein $3 / 4$ Jahr muss vorfinanziert werden bei der LE (Ländliche Entwicklung)."

„Großer bürokratischer Aufwand für Abrechnung und Dokumentation - Sollte einfacher gestaltet werden."

„Die Förderabwicklung ist sehr aufwendig, die sollte unbürokratischer sein, z.B. mittels Gemeinkostenpauschalen. Derzeit muss jede Kostenposition über Rechnung belegt werden. Innerhalb der genehmigten Kosten sollten Kosten zwischen Kostenpositionen verschiebbar sein - ist derzeit nicht möglich."

„Bei der TeilnehmerInnen-Förderung kann es bis zu 1 Jahr brauchen, bis die Teilnehmerlnnen das ausgelegte Geld zurück erhalten - das allerdings ohne fixe Zusage vor Kursbeitragsfälligkeit."

„Formalismus bei Antrag und Abwicklung ist enorm."

„Die AMA verlangt generell viel zu viele Daten bei der Abwicklung."

„Auf Länderebene ist die Abwicklung unterschiedlich. Der Bund sollte klare Abwicklungsrichtlinien haben." 
„Ein erheblicher Teil des Geldes wird für die Förderabwicklung benötigt - dieser Betrag fehlt dann bei der Erzielung von Wirkung."

„Was passiert mit den vielen Berichten und Statistiken? Die Abrechnung ist sowohl elektronisch als auch mehrfach in Papierform abzugeben - das kostet Geld und Platz!"

\subsubsection{Mitnahmeeffekte}

Der Mitnahmeeffekt bezeichnet die Inanspruchnahme finanzieller Förderungen als Belohnung für ein Verhalten, das auch ohne den zusätzlichen Anreiz stattgefunden hätte. Das Ausmaß eines Mitnahmeeffekts lässt sich empirisch nicht exakt ermitteln. Seitens der geförderten Veranstalter wurde in den Dialog-Interviews fast unisono argumentiert, dass ohne Veranstalterförderung die Bildungsveranstaltungen so teuer sein müssten, dass sich die Land- bzw. ForstwirtInnen diese nicht leisten könnten. Diesen Aussagen steht einerseits die Abbildung 93 entgegen, wonach immerhin rund $25 \%$ der Personen von Veranstaltern bei der Online-Umfrage angaben, keine Förderung erhalten zu haben. Weiters sagten jene Land- bzw. ForstwirtInnen, welche keine Kurse der Ländlichen Entwicklung besucht haben gemäß Abbildung 21, dass die Kurskosten das unwichtigste Kriterium war, das sie von Kursbesuchen abgehalten hat. Schließlich waren für all jene Land- bzw. ForstwirtInnen, welche Kurse der Ländlichen Entwicklung besucht haben, die Kurskosten das vorvorletzte Kriterium bei der Kurs-Auswahl - siehe Abbildung 54. All dies deutet darauf hin, dass die Mitnahmeeffekte vor allem bei Förderung von „Vorbereitung, Durchführung und Nachbereitung von Berufsbildungs- und Informationsmaßnahmen" sowie bei Teilnehmerlnnen-Förderungen erheblich sind. Eher geringe Mitnahmeeffekte sind hingegen bei der "Erstellung von Bedarfsstudien oder Konzepten" sowie bei der "Erstellung oder Ankauf von Unterlagen oder Hilfsmittel" zu vermuten, da in den Dialog-Interviews mehrfach nachvollziehbar dargelegt wurde, dass solche „Erstellungen“ die finanziellen Möglichkeiten der meisten Veranstalter - vielleicht mit Ausnahme der LFIs - überstiegen hätten.

Deshalb wird empfohlen, die Förderung von „Vorbereitung, Durchführung und Nachbereitung von Berufsbildungs- und Informationsmaßnahmen" sowie Teilnehmerlnnen-Förderungen stark zu reduzieren bzw. auslaufen zu lassen und dafür die Förderung von „Erstellung von Konzepten“ sowohl für Weiterbildungsmaßnahmen als auch für Beratungsmaßnahmen ebenso stark auszuweiten. Dies ist auch im Einklang mit der Ordnungspolitik der Europäischen Kommission primär die sogenannten vorwettbewerblichen (pre-competitive) Aktivitäten, wie Forschung, Entwicklung und Konzepterarbeitung, zu fördern und nicht die Erbringung von Dienstleistungen selbst, weil die Förderung der Erbringung von Dienstleistungen selbst stets eine marktverzerrende Wirkung hat zwischen jenen, deren Dienstleistungserbringung gefördert werden, und jenen, die eine solche Förderung nicht erhalten.

\subsubsection{Bundes/änderübergreifende Bildungsprojekte}

Bundesländerübergreifende Bildungsprojekte nehmen eine wichtige Sonderstellung aus zwei Gründen ein: Zum Einen war die Förderung der "Erstellung von Bedarfsstudien oder Konzepten" sowie der "Erstellung oder Ankauf von Unterlagen oder Hilfsmittel" nach Aussagen in den DialogInterviews ausschließlich im Rahmen bundesländerübergreifender Bildungsprojekte möglich. Zum Anderen konnten Landwirtschaftskammer-ferne Veranstalter Förderungen nur im Rahmen bundesländerübergreifender Bildungsprojekte in Anspruch nehmen, da nur das BMLFUW den Landwirtschaftskammer-fernen Veranstaltern diese Möglichkeit bot, wohingegen in den Bundesländern selbst fast ausschließlich die LFIs Förderungen beantragen konnten bzw. erhielten. Innovative Bildungsprojekte finden sich daher vorzugsweise in der Gruppe der bundesländerübergreifenden Bildungsprojekte, wohingegen die Bildungsprojekte in den Bundesländern eher durch die Förderung bestehender LFI-Angebote charakterisiert sind, mit vermuteten hohen Mitnahmeeffekten. 


\subsubsection{Erreichung der gewünschten Zielgruppen}

Bei den Zielgruppen fällt auf, dass bei der Online-Umfrage immerhin 23\% der Personen, welche an Kursen der Ländlichen Entwicklung teilgenommen haben, keine Land- oder ForstwirtInnen sind. Umgekehrt gaben bei der Online-Umfrage 13\% der Land- oder ForstwirtInnen an, seit 2007 keine Kurse der Ländlichen Entwicklung besucht zu haben. Gemäß Abb. 112 „Besuchte Kurse Vergleich nach Branche" zeigt sich indes, dass diese $23 \%$ der Personen vor allem Bildungsmaßnahmen M 331 und zu einem deutlich geringeren Teil Bildungsmaßnahmen M 111 in Anspruch genommen haben. Damit fällt die Erreichung der gewünschten Zielgruppen durchaus positiv aus.

Trotzdem muss darauf hingewiesen werden, dass gemäß Abb. 112 auch Personen, die keine Landoder ForstwirtInnen sind, an Bildungsmaßnahmen M 111 teilgenommen haben und damit eine Förderung erhalten haben, die ihnen gemäß Förderungsrichtlinien nicht zustand. Darüber hinaus ist anzumerken, dass die Zielgruppen bei der Bildungsmaßnahme $M 331$ nicht präzise genug beschrieben sind, um ex-post empirisch festzustellen, inwieweit bei M 331 die gewünschten Zielgruppen erreicht wurden.

Um daher für die nächste Periode die Begünstigung von Personen, die nicht zur Zielgruppe gehören, zu vermeiden, bietet sich zur Umsetzung der Verordnung (wie im Vorschlag zur Verordnung dargestellt) an, eine Förderungsabwicklung basierend auf dem Modell des Innovationsschecks, wie von der Österreichischen Forschungsförderungsgesellschaft (FFG) angewendet, zu realisieren. Dabei würden Personen der spezifizierten Zielgruppen zunächst einen "Wissenstransfer- und Innovationsscheck" beantragen. Nach einer Prüfung des Antrags auf Erfüllung der Förderkriterien erhält der Antragsteller den Scheck, der bei einem berechtigten Anbieter (Gewerbeschein, Ingenieurkonsulent, Universitäten, Fachhochschulen, etc.) von Kursen, Know-how oder Wissen, das den Zielen bzw. dem Programm der LE entspricht, eingelöst werden kann. Der Anbieter der Weiterbildung / des Wissenstransfers reicht nach Leistungserbringung den Scheck bei der Förderstelle ein, die die Auszahlung des Betrages an den Anbieter veranlasst. Eine Realisierung der Förderung mittels des vorgeschlagenen Modells trägt somit den Anforderungen der EU-Verordnung Rechnung:

„Die Unterstützung wird dem Anbieter der Ausbildung oder des sonstigen Wissenstransfers oder sonstiger Informationsmaßnahmen gewährt." (siehe Anhang III, Dok. [61], S. 45)

Auch in den Dialog-Interviews wurde diese Thematik mehrfach angesprochen:

„Teilnehmer-Förderung sollte bleiben, aber sollte erst nach der Teilnahme eingereicht werden."

„Förderabwicklung ist in jedem Bundesland anders, das ist mühsam."

„Es sollte länderübergreifende Veranstalterförderung geben, oder - einfacher - TeilnehmerlnnenFörderung."

„Die Veranstalter-Förderung führt dazu, dass Kurse keinen Wert haben."

\subsubsection{Genderspezifische Gestaltung der Bildungsmaßnahmen}

Wie aus den Auswertungen (siehe auch Kapitel 4.10 insbes. Abbildung 79) hervorgeht, gibt es noch großes Potenzial, die Zielgruppe der Frauen für Bildungsangebote zu gewinnen. Denn während Männer sich zu einem großen Teil schon als genug ausgebildet / wissend einstufen, und daher eine Inanspruchnahme von Bildung als nicht attraktiv gesehen wird, sehen die befragten Frauen eher eine Wissenslücke. Als wichtiger, hinderlicher Faktor bzgl. Teilnahme an den diversen Angeboten wurde seitens der Frauen "Zeit" identifiziert. In Zukunft gilt es daher, besonders auf 
die Bedürfnisse von Frauen einzugehen. Die hohen Antwortzahlen in den Kategorien "Termine sind ungünstig" und "Zu lange Anreise zum Veranstaltungsort" zeigen konkret Bereiche auf, wo rasch und unkompliziert reagiert werden kann, um mehr Frauen die Möglichkeit der Teilnahme zu bieten.

\subsubsection{Beratungs- und Veranstaltungs-Förderungslandschaft}

Von den 37 geförderten Veranstaltern erhielten 5, also 13,5\%, rund 61 \% der gesamten Veranstalterförderung. Dabei wird allerdings jedes LFI getrennt betrachtet. Nimmt man die 10 LFIs als eine, zusammengehörige Organisation, dann erhielt diese eine Organisation, also 3,6 \% der 28 geförderten Veranstalter, $67 \%$ der gesamten Veranstalterförderung.

Bei Beratungen - der neben Veranstaltungen ebenso wichtigen und von Organisationen und Unternehmen tendenziell stärker nachgefragten Form des Wissenstransfers - ist diese FörderungsVerteilung noch asymmetrischer, da ausschließlich die Beratungsleistungen der Landwirtschaftskammern gefördert werden.

Dass diese asymmetrische Verteilung der Fördermittel vor allem für jene Organisationen ein wichtiges Thema ist, welche keine oder im Vergleich mit den LFls eher kleine Förderungen erhielten, zeigen die Aussagen der Dialog-Interviews:

„Es gibt keine Projekteinreichungs- und -vergabetransparenz."

„Die Vergabe von Projekten sollte transparenter werden. Es fehlt sowohl ein Kriterienkatalog, als auch eine Entscheidungsbegründung. Förderprogramm ist für Neue schwer zugänglich - es gibt viele gute Projektideen, die Mangels Transparenz dem Ministerium nicht zur Kenntnis gelangen.

„Nicht-zertifizierte Bildungsträger können nicht direkt Anträge einreichen, außerdem braucht es eine Vorfinanzierung. Wird nicht per Anzahlung vorfinanziert, darf aber monatlich abgerechnet werden. Daher muss vorfinanziert werden, obwohl keine Gewinne erwirtschaftet werden dürfen."

„Eine Überlegung wäre, bei der Veranstalterförderung nur die Konzeption und Unterlagenerstellung zu fördern, und die Durchführung ausschließlich über die TeilnehmerInnen-Förderung abzuwickeln. Somit würde das System weniger wettbewerbsverzerrend zwischen verschiedenen Bildungsangeboten werden."

„Anbieterszene sollte geöffnet werden, damit mehr Markt entsteht, aber auch WIFI-Kurse besucht werden können."

„Die Förderung / das Programm sollte geöffnet werden, sodass mehr Organisationen / Veranstalter teilnehmen können."

"Die Landwirtschaftskammer als „Berater-Monopolist" hat nicht die Berater-Ressourcen um alle Betriebe zu beraten."

„Die Trennung zwischen Beratung und Qualifizierung ist künstlich."

„Auch Einzelberatung sollte möglich sein, statt weitere Broschüren oder Standardseminare."

"Leicht förderbar sind Veranstaltungen, aber gibt schon sehr viel Angebote. Beratung wäre manchmal besser, aber ist nicht förderbar."

„Bildung und Beratung muss eins sein im Sinne der Förderung, nicht so wie es jetzt ist."

„Auch Einzel-Know-How-Transfer für einzelne Betriebe sollte ermöglicht werden, nicht nur Kurse und Seminare für Gruppen." 
„Es darf zu keiner Benachteiligung von privaten Beratern kommen!“

Sollten die Landwirtschaftskammern und die LFIs tatsächlich die, im Sinne der Ziele der LE überwiegend besten Bildungs- und Beratungsprojekte bei den fördergebenden Stellen eingereicht haben, wäre diese Förderungs-Verteilung gerechtfertigt. Nachdem jedoch die FörderungsVerteilung von Beratung und Veranstaltung im land- und forstwirtschaftlichen Sektor im Vergleich mit anderen Wirtschaftssektoren ungewöhnlich asymmetrisch ist, liegt die Vermutung nahe, dass die Landwirtschaftskammern und die LFIs bei der Zuteilung von Bildungs- und noch mehr bei der Zuteilung von Beratungs-Fördermittel bevorzugt wurden.

Wenn ein Anbieter auf Grund von erhaltenen Fördermitteln seine Leistungen deutlich billiger anbieten kann als die Mitbewerber, führt dies zu Marktverzerrungen. In der Land- und Forstwirtschaft sind diese Marktverzerrungen im Bereich Bildung sowie Beratung im Vergleich mit anderen Wirtschaftssektoren hoch. Dies wirkt sich nachteilig auf die Vielfalt von Bildungs- sowie Beratungsangeboten für die Land- und Forstwirtschaft aus.

Eine wichtige Ursache für die bevorzugte Zuteilung von Fördermitteln an Landwirtschaftskammern und LFIs dürfte sein, dass die Fördergeber als formale Voraussetzung den Nachweis eines zertifizierten Qualitätsmanagementsystems verlangen. So sinnvoll dies für große Industriebetriebe ist, so wenig bewährt sich ein solches zertifiziertes QM-System bei der Überprüfung, ob eine Organisation, welche Beratungs- oder Weiterbildungsleistungen anbietet, das entsprechende Wissen hat, denn im Bereich wissensintensiver Dienstleistungen ist primär das Wissen der Personen bedeutsam und eher wenig, ob mittels eines Qualitätsmanagementsystems die Qualität in einer Organisation geprüft und verbessert wird.

Die überwiegende Anzahl an Unternehmen, welche Beratungs- oder Weiterbildungsleistungen anbieten, sind Kleinstunternehmen mit weniger als 10 Beschäftigten, für die die Implementierung eines zertifizierten Qualitätsmanagementsystems nicht nur teuer ist, sondern auch keinen Beitrag zur Verbesserung der Expertise des Unternehmens leistet. Deshalb scheuen die meisten ExpertInnen-Unternehmen im Bereich Beratung und Weiterbildung eine solche Zertifizierung und operieren lieber in Märkten, wo dies nicht Bedingung ist.

Laut Europäischer Kommission wird für das Programm LE kein zertifiziertes Qualitätsmanagementsystem verlangt; es genügt die landesübliche Konzession für Beratungs- oder Weiterbildungsleistungen als formale Förderungsvoraussetzung: „Die Anbieter von Wissenstransfer und Informationsdiensten müssen über die geeigneten Fähigkeiten in Form von qualifiziertem Personal und regelmäßigen Schulungen zur Durchführung dieser Aufgabe verfügen." (siehe Anhang III, Dok. [61], S. 46). In Österreich könnten dies der entsprechende Gewerbeschein bzw. die Ingenieurkonsulenten der Fachgruppen Natürliche Ressourcen, Raumplanung, Landschaftsplanung und Geographie sowie Wasserwirtschaft sein. Wesentlich ist, dass ein Formalkriterium, welches keine Aussage über die Relevanz sowie Qualität eingereichter Bildungs- oder Beratungs-Projekte zulässt aber Kleinstunternehmen mit hoher Expertise benachteiligt, zugunsten der Prüfung von Relevanz und Qualität eingereichter Projektanträge abgeschafft wird.

Es wird daher empfohlen, bei der Auswahl von Projekten in LE 2020 das Formalkriterium "zertifiziertes QM-System " abzuschaffen und durch das Formalkriterium Gewerbeschein, Ingenieurkonsulent, Land- und Forstwirt bzw. Wissenschaftseinrichtung (Universitäten und Fachhochschulen) zu ersetzen.

Weiters wird empfohlen, über entsprechende, regelmäßig stattfindende und stichtagsbezogene öffentliche Ausschreibungen zur Einreichung von Projekten in den Bereichen Bildung, Wissenstransfer und Innovation aufzufordern und dann die, im Sinne der LE 2020-Ziele bestgeeignetsten Projekte durch eine ExpertInnen-Jury zur Förderung auszuwählen und die Ablehnung von Projek- 
ten so zu begründen, dass die Förderwerber davon lernen und es beim nächsten Mal besser machen können. Orientieren könnte sich diese Vorgehensweise bei der Auswahl förderbarer Projekte an der 2004 gegründeten Österreichischen Forschungsförderungsgesellschaft.

Die Umsetzung dieser beiden Empfehlungen würde auch dem, in einem früheren Evaluierungsbericht formulierten Ziel Rechnung tragen: „Es wäre gerade auch in Hinblick auf ein möglichst vielfältiges und unterschiedliches Bildungsangebot, das immer komplexere Problemstellungen zu berücksichtigen hat, wünschenswert, den Zugang zu Fördermitteln auf einen erweiterten Kreis an Bildungsanbietern auszuweiten." - siehe Anhang III, Dok. [24], S. 59 ).

All diese Aspekte passen auch mit der Aussage aus der Online-Umfrage zusammen - siehe Kapitel 4.4 (Abbildung 54) -, wonach für die befragten Land- bzw. ForstwirtInnen „Bekanntheit und Image des Veranstalters" am wenigsten hingegen "Qualifikation und Kompetenz der TrainerInnen" äuBerst wichtig sind.

Klar ist, dass die empfohlene Marktöffnung bei Beratung und Weiterbildung nicht unbedingt auf Gegenliebe bei den voraussichtlichen Verlierern einer solchen Marktöffnung stoßen wird, auf der anderen Seite werden die Land- und ForstwirtInnen davon enorm profitieren, insbesondere hinsichtlich der Wettbewerbsfähigkeit und damit auch der Einkommenssituation - zwei der Kriterien, bei denen gemäß Abbildung 24 die Veränderung seit 2007 nicht ganz so positiv war, wie bei den meisten anderen Kriterien.

In den Dialog-Interviews wurde all dies so geäußert:

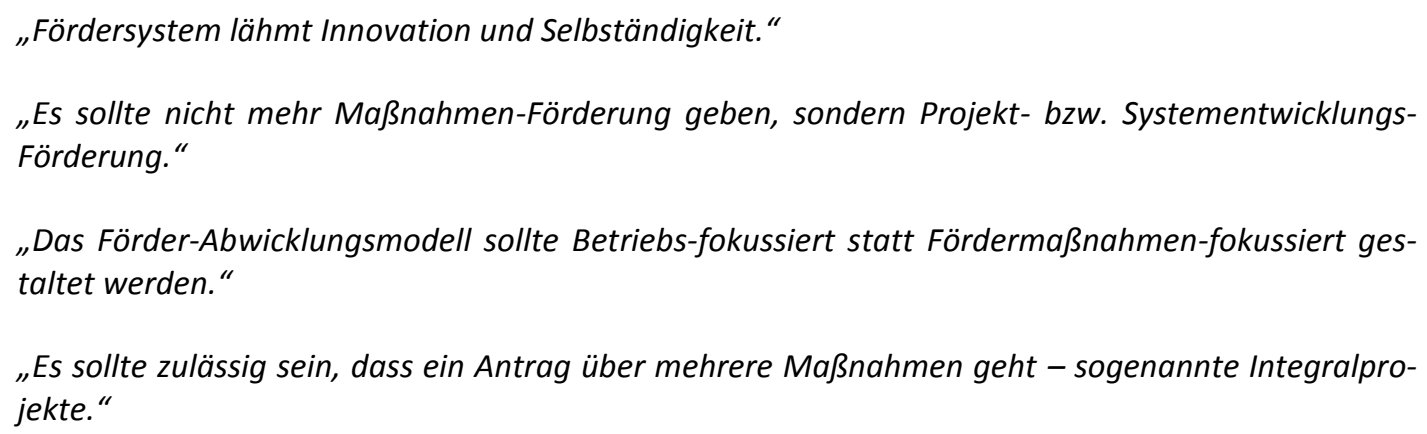

\subsubsection{Verbreitung wissenschaftlicher Erkenntnisse}

Beim Vergleich mit Programmen der LE anderer Europäischer Länder wie bspw. Schweden (siehe auch Anhang III, Dok. [50]) fällt auf, dass besonders in der Maßnahme 111 der Teilaspekt betreffend die Verbreitung wissenschaftlicher Erkenntnisse und innovativer Verfahren keine gesonderte Stelle einnimmt bzw. in der Umsetzung des Programms in den Hintergrund tritt. In Hinblick auf den, im Programm für die Periode 2014-2020 wichtigen neuen Schwerpunkt Wissenstransfer und Innovation sollte indes besonderes Augenmerk auf folgende Aspekte gelegt werden: Schaffung von "Meeting-Places" zum Austausch von Informationen zwischen Wissenschaftlern und landund forstwirtschaftlichen Betrieben; Erstellung einer Wissensdatenbank bzw. Webplattform, um den in der Land- und Forstwirtschaft tätigen Personen direkten Zugriff zu Ergebnissen aus der Forschung zu ermöglichen; insbesondere aber auch die Öffnung der Beratungs- und Veranstaltungs-Förderungslandschaft wie in Kapitel 5.1.1.7 dargelegt.

In den Evaluation Cafés gab es dazu eine Reihe von Anregungen:

„Lehre - Forschung - Beratung - Landwirte verknüpfen"

„Koordination von Wissenschaft und Bildungsanbieter; Netzwerk schaffen" 
„Wissenstransfer zwischen Forschung - Bildung - Beratung - Praxis: Austausch / Kooperation in beide Richtungen"

"Nutzenorientierter, systematischer Austausch und Zusammenarbeit zwischen Forschung und Praxis"

\subsubsection{Austausch und Vergleich mit anderen EU-Ländern}

Generell wird ein systematischer Austausch und Benchmarking sowohl auf der Ebene der Landund ForstwirtInnen, als auch auf der Ebene der Beratungs- und Weiterbildungsanbieter sowie auf der Ebene der Förderstellen mit anderen Ländern der EU empfohlen, denn Wissenstransfer und Innovation erfolgt umso effektiver, je größer der Wissenspool ist, aus dem geschöpft wird.

Auch in den Dialog-Interviews wurde auf die Bedeutung des "globalen Denkens, lokalen Handelns" hingewiesen:

"Exkursionen werden nicht mehr gefördert, das ist ganz schlecht, weil Betriebsbesuche im Ausland z.B. Schweiz besonders lernträchtig sind und besonders gut ankommen."

„Junge Bauern sollten viel mehr in Richtung Auslands-Praktika (bei exzellenten Betrieben) gefördert werden."

In LE 2020 sollten deshalb auch Projekte gefördert werden, welche den Wissenstransfer von anderen als ausschließlich österreichischen Wissenschafts-Organisationen zu österreichischen landund forstwirtschaftlichen Betrieben zum Gegenstand haben.

\subsubsection{Wissenstransfer - Themengebiete}

Hinweise für die zukünftigen, den Bedürfnissen der Zielgruppe angepassten Themengebiete des Wissenstransfers geben die zuvor in Kapitel 4 präsentierten Abbildungen 47, 49, 51, 100. Bei der Auswahl der förderungswürdigen Themengebiete ist es wichtig diese gemeinsam mit den Landbzw. ForstwirtInnen festzulegen, um unterrepräsentierte Querschnittsthemen - derzeit z.B. „Ressourcen-Schonung / guter Boden“ - zu identifizieren.

Aussagen aus den Dialog-Interviews:

„Bildungsangebote sind oft von oben > es kommt jemand, der einem sagt, wie es geht."

„Für Jungbauern/ -bäuerinnen sollte es mehr Bildungsangebote zum Thema, wie der Hof erhalten werden kann geben."

„Es sollte mehr zum Thema „Bauer als Unternehmer" geben."

"Generell braucht es mehr Bildungsangebote zu Hofübergabe/-übernahme - auch an andere (Familienexterne)."

"Was fehlt ist eine Segmentierung des Bildungsangebotes entsprechend den Bedürfnissen."

„Für die Zukunft wäre es gut, sektorübergreifende Angebote zu haben - insbesondere entlang der Wertschöpfungskette und regional, z.B. Wald > Holz > Tischlerei > Möbel."

"Noch mehr Fokus auf Wald als wichtiges ökologisches System." 
Anregung aus den Evaluation Cafés:

"Bedarfsgerechte Entwicklung von Bildungs- und Beratungsangeboten"

\subsubsection{Wissenstransfer - Lernformen}

\subsubsection{Exkursionen}

Exkursionen wurden in der LE zuletzt nicht mehr gefördert. Aus Abbildung 62 in Kapitel 4.5 geht jedoch hervor, dass es sich dabei um eine, von den Land- bzw. ForstwirtInnen als äußerst nützlich angesehene Lernform handelt. Im Vorschlag zur EU-Verordnung für die Programmperiode 20142020 heißt es dazu: „Der Wissenstransfer und die Informationsaktionen sollten nicht nur über herkömmliche Schulungen erfolgen, sondern den Bedürfnissen der ländlichen Akteure angepasst sein. Daher sollten Workshops, Coaching, Demonstrationstätigkeiten, Informationsaktionen, aber auch kurzzeitige Austausch und Besuchsprogramme für Landwirte unterstützt werden." (siehe Anhang III, Dok. [61], S. 14)

Die nachstehend angeführten Aussagen aus den Dialog-Interviews sprechen u.a. ebenfalls die Thematik der zuletzt nicht mehr geförderten Exkursionen und deren Wichtigkeit für die Landbzw. ForstwirtInnen an:

„Wir wissen, dass Bauern am Liebsten von Bauern lernen!"

„Es gibt große Seminarmüdigkeit, vor allem vom LFI, gleichzeitig Kürzungen bei Exkursionen."

„Netzwerk-Bildung sollte mehr gefördert werden z.B. über Arbeitskreise, Workshops, Stammtische, etc."

„Es ist wichtig, bestimmte Maßnahmen, wo es um Innovation geht zu 100\% zu fördern. Bei Seminaren wäre es möglich die Förderquote zu reduzieren."

"Arbeitskreise sind am wirkungsvollsten."

"Die Kombination zwischen Fachbildung und Persönlichkeitsbildung ist besonders wirksam im Sinne der Weiterentwicklung eines landwirtschaftlichen Betriebes."

„Bauern, vor allem Männer, schätzen am Meisten Persönlichkeitsbildung im Rahmen einer Fachbildung, wie z.B. Unternehmensführung."

„Arbeitskreise zur Betriebsauswertung führen dazu, dass schwächere Betriebe von den besseren Betrieben lernen."

„Was fehlt sind moderne Kommunikationsarchitekturen statt klassische Lehrer-zentrierte Veranstaltungen."

„Von-einander-lernen verstärken!"

„Arbeitskreise waren sehr gut! Das ist „an der Basis lernen" und sollte verstärkt werden - mit begleitendem Coaching."

"Arbeitskreis hat mehr gebracht, als die Meisterausbildung."

"Bildung und Beratung ist zu sehr getrennt. Es sollte mehr Bildungsmaßnahmen in Form von Beratung geben, z.B. kleine Arbeitskreise." 
„Wertschöpfung im ländlichen Raum muss gefördert und verstärkt werden - über Wertschöpfungsketten vom (Lebensmittel-) Rohstoff zum fertigen Produkt."

\subsubsection{Beratung}

Wie bereits in Kapitel 3.1.1.7 begründet, muss Beratung und Weiterbildung als zusammengehörig und einfach als zwei verschiedene Formen des Wissenstransfers gesehen werden. Darüber hinaus ist das Wissen, welches für eine moderne, innovative Land- und Forstwirtschaft erforderlich ist, bereits viel zu aufgefächert und diversifiziert, als dass dies bei den Landwirtschaftskammern alleine angesiedelt werden kann.

Deshalb wird empfohlen, dass die Förderung

- sowohl von Beratung als auch von Bildung gleich gestaltet wird,

- offen für alle Organisationen wird, welche zur Erbringung von Beratungs- oder BildungsLeistungen nach österreichischem Recht befugt bzw. konzessioniert sind, und

- der vorhandenen Asymmetrie bei der Verteilung der Fördermittel bei Beratung und Bildung in der Land- und Forstwirtschaft als Angebotsvielfalt-hemmend entgegenwirkt.

Zugänglichkeit von Wissen für Land- und ForstwirtInnen bedeutet vor allem Zugänglichkeit zu allen Anbietern von Wissen ohne Preis- und damit Marktverzerrungen durch FörderungsUngleichverteilung.

In den Dialog-Interviews kam dies klar zum Ausdruck:

„Die Kammer ist die offizielle Bauern-Beratung, die auch gefördert wird, aber außerhalb der EUFörderung. Die Berater sind alle Kammer-Angestellte."

„Die Landwirtschaftskammer als „Berater-Monopolist" hat nicht die Berater-Ressourcen um alle Betriebe zu beraten."

„Die Trennung zwischen Beratung und Qualifizierung ist künstlich."

„Auch Einzelberatung sollte möglich sein, statt weitere Broschüren oder Standardseminare."

"Leicht förderbar sind Veranstaltungen, aber gibt schon sehr viel Angebote. Beratung wäre manchmal besser, aber ist nicht förderbar."

„Bildung und Beratung müssen stärker zusammengebracht werden."

"Bildung und Beratung muss eins sein im Sinne der Förderung, nicht so wie es jetzt ist."

„Beratung sollte auch gefördert werden."

„Auch Einzel-Know-How-Transfer für einzelne Betriebe sollte ermöglicht werden, nicht nur Kurse und Seminare für Gruppen."

„Gute Maßnahmen hängen mit guter Beratung zusammen."

„Es darf zu keiner Benachteiligung von privaten Beratern kommen!"

„Konzession für Berater müssten genügen" 


\subsection{Strukturierung der Datenbasis für Auswertungen und Ergebnisse}

Im VO-Vorschlag LE2020 steht: „Um sicherzustellen, dass Informationen auf EU-Ebene aggregiert werden können, sollte vorgesehen werden, dass eine Reihe von gemeinsamen Indikatoren Teil des Systems ausmacht. Schlüsselinformationen über die Durchführung der Entwicklungsprogramme für den ländlichen Raum sollten elektronisch aufgezeichnet und gespeichert werden, um die Datenaggregation zu erleichtern. Die Begünstigten sollten daher die Mindestangaben übermitteln müssen, die für das Monitoring und die Evaluierung erforderlich sind." - siehe Anhang III, Dok. [61], S. 27).

Dem ist grundsätzlich wenig hinzuzufügen. Ergänzend wird empfohlen, dass jedes, im Bereich Wissenstransfer und Innovation geförderte Projekt bei Projektabschluss gemeinsam mit der finanziellen Projektabrechnung einen, von allen Projekt-Stakeholdern ausgefüllten OnlineFragebogen abliefern muss. Dieser Online-Fragebogen sollte für ganz Österreich einheitlich sein und von allen, am jeweiligen Projekt mitwirkenden bzw. daraus Nutzen ziehenden Personen ausgefüllt werden, d.h. sowohl von allen LeistungserbringerInnen (BeraterInnen, TrainerInnen, ...) als auch von allen LeistungsempfängerInnen (Land- und ForstwirtInnen).

Inhalt des Online-Fragebogens könnte eine abgespeckte Variante des für diese Studie entwickelten Fragebogens sein, wobei aber die Wirkungsindikatoren den Zielen von LE 2020 entsprechend angepasst werden müssten.

Wichtig ist dabei die Erkenntnis, dass proaktive Steuerung nicht durch Wirkungsindikatoren und deren Auswertung erfolgen kann, weil diese stets erst nach Projektende zur Verfügung stehen sondern durch entsprechende Ausschreibungen mit klaren Ausschreibungszielen und durch die sachkundige Auswahl jener Projekte, die den Ausschreibungszielen am besten entsprechen und eine hohe Erfolgswahrscheinlichkeit der Zielerreichung aufweisen.

Steuern bedeutet, ein angestrebtes Ziel bestmöglich zu erreichen. Dazu ist es erforderlich, klare und operationalisierte qualitative als auch quantitative Ziele von den Projekten zu erhalten, bevor diese gefördert werden, zu beurteilen, inwiefern diese Projektziele dazu beitragen, die Programmziele zu erreichen und schließlich, bei Projektende einen Soll-Ist-Vergleich bezüglich der Projektziele empirisch zu ermitteln, um daraus Erkenntnisse zu gewinnen - sowohl für die Fördergeber als auch für die Fördernehmer.

\subsection{Wirkungsindikatoren}

Alle Projekte, welche zur Förderung eingereicht werden, sollten klare qualitative und quantitative Projektziele haben, welche dem Fördergeber vorgelegt werden. Diese Projektziele wiederum müssen sich den Zielen einer bestimmten Ausschreibung unterordnen. Die Ausschreibungsziele wiederum müssen sich den Zielen des Programms LE 2020 unterordnen. Wirkungen eines Projektes sollten daher stets nur in Bezug auf die Ausschreibungsziele und damit auf die Programm-Ziele ermittelt werden, da diese Ziele ja die intendierten Wirkungen festlegen. Alle anderen Wirkungen sind nicht intendiert und daher als Nebenwirkungen zu betrachten, welche im Sinne zielorientierten Handelns nebensächlich sind. Sinn und Zweck der Wirkungsindikatoren ist daher, die Wirkungen zu ermitteln, nicht die Nebenwirkungen.

Bei komplexen, immateriellen Zielen ist es grundsätzlich unmöglich Wirkungsindikatoren zu spezifizieren, welche in Einheiten wie EURO oder Anzahl Teilnehmerlnnen gemessen werden können. Das Erreichen von Zielen wie Chancengleichheit oder Wettbewerbsfähigkeit ist nicht dadurch ermittelbar, dass die Kosten, die TeilnehmerInnen-Anzahl und deren Geschlecht gemeldet werden. 
Wirkungsindikatoren darüber, inwieweit ein Projekt, eine Ausschreibung, ein Programm zur Erreichung vorab definierter Ziele beigetragen hat, können daher nur durch Einschätzungen der an einem Projekt beteiligten Personen ermittelt werden, so wie dies in dieser Studie erfolgt ist.

Die Wirkungsindikatoren für diese Studie wurden auf Basis der Ziele der Bildungsmaßnahmen der LE 07-13 erarbeitet und entsprechend ausgewertet - siehe insbesondere alle Wirkungsindikatoren, wie sie in den Abbildungen in Kapitel 4 aufscheinen.

Auf Basis der Ziele des Wissenstransfers und Innovation von LE 2020 sollten daher die Wirkungsindikatoren erarbeitet werden, so wie dies in dieser Studie für die Bildungsmaßnahmen von LE 0713 gemacht wurde, und dann diese Wirkungsindikatoren für jedes abgeschlossene, geförderte Projekt erhoben werden, so wie dies in Kapitel 5.2 dargelegt ist.

So wie beim Austesten neuer Medikamente in der Medizin, sollten dieselben Wirkungsindikatoren auch bei einer Kontrollgruppe von Land- und ForstwirtInnen erhoben werden, die aus Personen bestehen muss, die in das jeweilige Projekt nicht involviert waren.

Die Auswertungen dieser Wirkungsindikatoren pro Projekt und pro Ausschreibung sollten dann dazu verwendet werden, die zukünftige Auswahl von Projekten und die Beschreibung zukünftiger Ausschreibungen dahingehend zu modifizieren, dass Programmziele, die nicht so gut erreicht werden, an Bedeutung gewinnen, und Ziele, die gut erreicht werden, an Bedeutung verlieren. Dies ist die Quintessenz von ex post Steuerung durch Wirkungsindikatoren! 


\section{ANHÄNGE}




\section{ANHANG I-ABKÜRZUNGSVERZEICHNIS}

BMLFUW

LFI

M 111

M 331

$\mathrm{N}$
Bundesministerium für Land- und Forstwirtschaft, Umwelt und Wasserwirtschaft Ländliches Fortbildungsinstitut Maßnahme 111 - Berufsbildung und Information Maßnahme 331 - Ausbildung und Information Bezeichnet jeweils die Grundgesamtheit der Befragten 


\section{ANHANG II - ABBILDUNGSVERZEICHNIS}

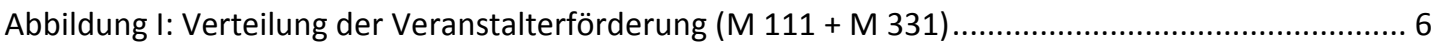

Abbildung II: Verteilung der Veranstalterförderung (M $111+$ M 331) - LFIs zusammengefasst............ 6

Abbildung III: Veränderungen für Land- bzw. ForstwirtInnen seit 2007 - nach Teilnahme.................. 7

Abbildung IV: Empfundener Nutzen der Bildungsangebote - M 111 \& M 331 ................................. 8

Abbildung V: Empfundener Nutzen der Bildungsangebote - Agrar-Umwelt und Naturschutz .............. 8

Abbildung VI: Empfundener Nutzen der Bildungsangebote - Forstbildungsmaßnahmen .................... 9

Abbildung VII: Chancengleichheit - Gründe für Nicht-Teilnahme ............................................... 9

Abbildung 1: Maßnahme 111 (Berufsbildung und Information) - Gesamtförderung ........................ 15

Abbildung 2: Maßnahme 111 (Berufsbildung und Information) - Veranstalterförderung .................. 16

Abbildung 3: Maßnahme 331 (Ausbildung und Information) - Gesamtförderung ............................ 17

Abbildung 4: Maßnahme 331 (Ausbildung und Information) - Veranstalterförderung ...................... 18

Abbildung 5: TeilnehmerInnen am Fragebogen (gesamt) ....................................................... 20

Abbildung 6: Teilnehmerlnnen aus anderen Branchen - Besuch von Bildungsangeboten der LE........ 20

Abbildung 7: Aufteilung der TeilnehmerInnen (gesamt) nach Gender ........................................... 21

Abbildung 8: TeilnehmerInnen (gesamt) nach Altersgruppe ..................................................... 21

Abbildung 9: Teilnehmerlnnen (gesamt) nach Bundesland ......................................................... 22

Abbildung 10: TeilnehmerInnen (Land- / ForstwirtInnen) nach Bundesland ................................... 22

Abbildung 11: TeilnehmerInnen (KursbesucherInnen aus anderen Branchen) nach Bundesland ....... 23

Abbildung 12: TeilnehmerInnen (öffentliche Dienststellen und Veranstalter) nach Bundesland ........ 23

Abbildung 13: Aufteilung der TeilnehmerInnen (gesamt) nach Schulbildung.................................... 24

Abbildung 14: Aufteilung der Land- bzw. ForstwirtInnen nach Gender ............................................ 26

Abbildung 15: Aufteilung der Land- bzw. ForstwirtInnen nach Altersgruppe ................................... 26

Abbildung 16: Aufteilung der Land- bzw. ForstwirtInnen nach Schulbildung ................................. 27

Abbildung 17: Aufteilung der Land- bzw. ForstwirtInnen nach Haupt- und Nebenerwerb ................ 27

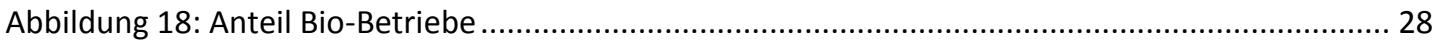

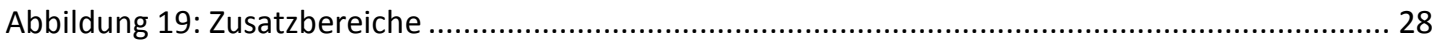

Abbildung 20: Teilnahme an Bildungsveranstaltungen der LE 07-13 ............................................ 29

Abbildung 21: Begründung für die Nicht-Teilnahme an Bildungsangeboten der LE .......................... 29

Abbildung 22: Begründung für die Nicht-Teilnahme an Bildungsangeboten nach höchster abgeschlossener Schulbildung ................................................................................... 30

Abbildung 23: Veranstalter, bei welchen die Land- bzw. ForstwirtInnen Kurse der LE besucht haben

Abbildung 24: Veränderungen für Land- bzw. ForstwirtInnen seit 2007 - nach Bildungsmaßnahme . 33

Abbildung 25: Veränderungen für Land- bzw. ForstwirtInnen seit 2007 - nach Teilnahme................. 34

Abbildung 26: Gegenüberstellung der Einschätzungen zwischen 2007 und 2013 nach Gender ......... 35

Abbildung 27: Veränderungen bzgl. Wettbewerbsfähigkeit seit 2007 - M 111 \& M 331 ................... 36

Abbildung 28: Veränderungen bzgl. Wettbewerbsfähigkeit seit 2007 - Agrar- Umwelt und Naturschutz......

Abbildung 29: Veränderungen bzgl. Wettbewerbsfähigkeit seit 2007 - Forstbildungsmaßnahmen ... 37

Abbildung 30: Veränderungen bzgl. Wettbewerbsfähigkeit seit 2007 - keine Bildungsangebote besucht.

Abbildung 31: Veränderungen bzgl. Wettbewerbsfähigkeit seit 2007 - Gender ............................ 38

Abbildung 32: Veränderungen bzgl. nachhaltiger Wirtschaftsweise seit 2007 - M 111 \& M 331........ 38

Abbildung 33: Veränderungen bzgl. Naturschutz- und umweltrelevantem Denken und Handeln seit 2007 - M 111 \& M 331.

Abbildung 34: Veränderungen bzgl. erneuerbarer Energie seit 2007 - M 111 \& M 331.................... 39

Abbildung 35: Veränderungen bzgl. nachhaltiger Wirtschaftsweise seit 2007 - Agrar- Umwelt und Naturschutz ................................................................................................... 40

Abbildung 36: Veränderungen bzgl. Naturschutz- und umweltrelevantem Denken und Handeln seit 2007 - Agrar- Umwelt und Naturschutz ........................................................... 40

Abbildung 37: Veränderungen bzgl. erneuerbarer Energie seit 2007 - Agrar- Umwelt und Naturschutz 
Abbildung 38: Veränderungen bzgl. nachhaltiger Wirtschaftsweise seit 2007 Forstbildungsmaßnahmen 41

Abbildung 39: Veränderungen bzgl. Naturschutz- und umweltrelevantem Denken und Handeln seit 2007 - Forstbildungsmaßnahmen

Abbildung 40: Veränderungen bzgl. erneuerbarer Energie seit 2007 - Forstbildungsmaßnahmen......42

Abbildung 41: Nachhaltige Wirtschaftsweise - Vergleich ................................................................43

Abbildung 42: Naturschutz- und umweltrelevantem Denken und Handeln - Vergleich .....................43

Abbildung 43: Erzeugung und Nutzung erneuerbarer Energie - Vergleich ......................................44

Abbildung 44: Veränderungen bzgl. nachhaltiger Wirtschaftsweise seit 2007 - Gender....................44

Abbildung 45: Veränderungen bzgl. Naturschutz- und umweltrelevantem Denken und Handeln seit 2007 - Gender.

Abbildung 46: Veränderungen bzgl. erneuerbarer Energie seit 2007 - Gender..................................45

Abbildung 47a: Bewertung des Bildungsangebotes (absolut) - M 111 \& M 331 ..............................47

Abbildung 47b: Bewertung des Bildungsangebotes (in \%) - M 111 \& M 331 ....................................48

Abbildung 48: Besuchte Kurse - M 111 \& M 331 ..........................................................................49

Abbildung 49a: Bewertung des Bildungsangebotes (absolut) - Agrar-Umwelt und Naturschutz .........50

Abbildung 49b: Bewertung des Bildungsangebotes (in \%) - Agrar-Umwelt und Naturschutz ..............51

Abbildung 50: Besuchte Kurse - Agrar-Umwelt und Naturschutz ...................................................52

Abbildung 51a: Bewertung des Bildungsangebotes (absolut) - Forstbildungsmaßnahmen .................53

Abbildung 51b: Bewertung des Bildungsangebotes (in \%) - Forstbildungsmaßnahmen .....................54

Abbildung 52: Besuchte Kurse - Forstbildungsmaßnahmen ..........................................................55

Abbildung 53: Informationsquellen $\mathrm{zu}$ den Bildungsangeboten - Vergleich nach

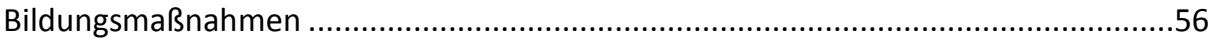

Abbildung 54: Auswahlkriterien - Vergleich nach Bildungsmaßnahmen ........................................57

Abbildung 55a: Bewertung des Bildungsangebotes (absolut) - Gender...........................................58

Abbildung 55b: Bewertung des Bildungsangebotes (in \%) - Gender................................................59

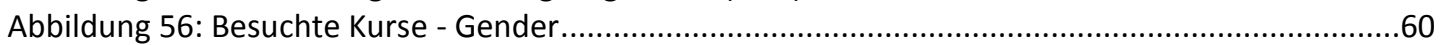

Abbildung 57: Informationsquellen zu den Bildungsangeboten - Gender ....................................61

Abbildung 58: Auswahlkriterien - Vergleich nach Bildungsmaßnahmen ..........................................62

Abbildung 59: Empfundener Nutzen der Bildungsangebote - M 111 \& M 331 ................................63

Abbildung 60: Empfundener Nutzen der Bildungsangebote - Agrar-Umwelt und Naturschutz ...........63

Abbildung 61: Empfundener Nutzen der Bildungsangebote - Forstbildungsmaßnahmen ..................64

Abbildung 62: Lernformen - Vergleich nach Bildungsmaßnahmen .................................................65

Abbildung 63: Empfundener Nutzen der Bildungsangebote - Gender................................................66

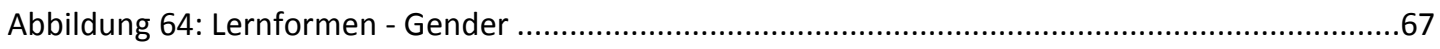

Abbildung 65: Nicht-agrarische Bereiche - M 111 \& M 331 .........................................................68

Abbildung 66: Nicht-agrarische Bereiche - Agrar-Umwelt und Naturschutz...................................68

Abbildung 67: Nicht-agrarische Bereiche - Forstbildungsmaßnahmen..........................................69

Abbildung 68: Nicht-agrarische Bereiche - Forstbildungsmaßnahmen............................................69

Abbildung 69: Nicht-agrarische Bereiche - Gender ....................................................................70

Abbildung 70: Veränderung seit 2007 im Detail - Vergleich nach Teilnahme .......................................71

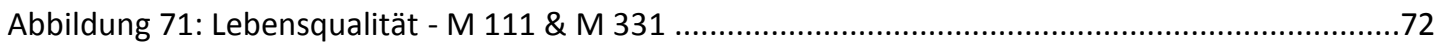

Abbildung 72: Lebensqualität - Agrar-Umwelt und Naturschutz ..................................................73

Abbildung 73: Lebensqualität - Forstbildungsmaßnahmen ........................................................73

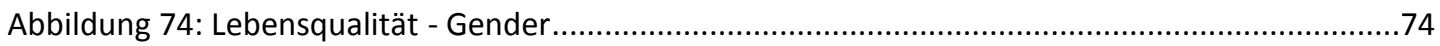

Abbildung 75: Regionale Entwicklung - nicht-agrarische Bereiche ................................................74

Abbildung 76: Regionale Entwicklung - Bildungsangebote ..........................................................75

Abbildung 77: Regionale Entwicklung - Vergleich nach Bildungsmaßnahmen ....................................76

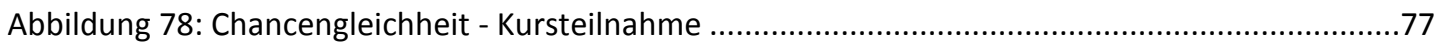

Abbildung 79: Chancengleichheit - Gründe für Nicht-Teilnahme .......................................................77

Abbildung 80: Schulbildung nach Gender - Frauen ....................................................................78

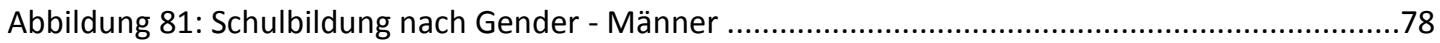

Abbildung 82: Aufteilung Agrar- und Waldpädagogik....................................................................79

Abbildung 83: Aufteilung nach Betrieb / Organisation - Agrar- und Waldpädagogik ............................80

Abbildung 84: Aufteilung Agrar- \& Waldpädagogik - TeilnehmerInnen aus anderen Branchen...........80

Abbildung 85: Empfundener Nutzen - Agrar- und WaldpädagogInnen gesamt..................................81

Abbildung 86: Empfundener Nutzen - Seminarbäuerinnen .........................................................81 


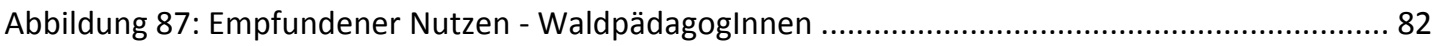

Abbildung 88: Empfundener Nutzen - Schule am Bauernhof-Betriebe ........................................ 82

Abbildung 89: Empfundener Nutzen - Land- bzw. ForstwirtInnen ................................................ 83

Abbildung 90: Empfundener Nutzen - TeilnehmerInnen aus anderen Branchen ............................... 83

Abbildung 91: besuchte Kurse - Agrar- und Waldpädagogen .................................................... 84

Abbildung 92: Veränderungen seit 2007- Agrar- und Waldpädagogen ............................................. 85

Abbildung 93: Anteil der Veranstalter mit erhaltener Veranstalterförderung................................. 85

Abbildung 94: Arbeitsaufwand nach Tätigkeit - Veranstalter gesamt........................................... 86

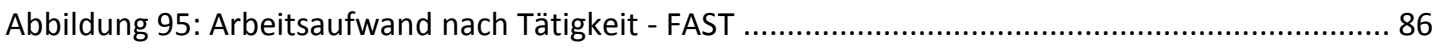

Abbildung 96: Arbeitsaufwand nach Tätigkeit - LFI .................................................................. 87

Abbildung 97: Arbeitsaufwand nach Tätigkeit - Natur- \& Umweltschutz ....................................... 87

Abbildung 98: Arbeitsaufwand nach Tätigkeit - öffentliche Dienststellen ........................................ 88

Abbildung 99: Arbeitsaufwand nach Tätigkeit - Sonstige Bildungsveranstalter................................ 88

Abbildung 100a: Bewertung des Bildungsangebote (absolut) - Vergleich Veranstalter / öffentliche

Dienststellen vs. LW / FW ............................................................................ 89

Abbildung 100b: Bewertung des Bildungsangebotes (in \%) - Vergleich Veranstalter / öffentliche

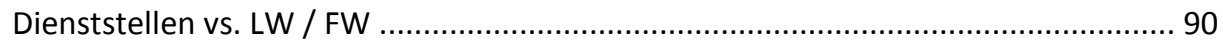

Abbildung 101: Themen der Bildungsangebote - Veranstalter im Vergleich ................................... 91

Abbildung 102: Angebot und Nachfrage an Bildungsangeboten .................................................... 92

Abbildung 103: Bewerbung der Bildungsangebote - Veranstalter im Vergleich ................................ 93

Abbildung 104: Bewerbung der Bildungsangebote und Wahrnehmung seitens der LW /FW ............ 94

Abbildung 105: Einschätzung der Veränderung seit 2007 - Veranstalter im Vergleich....................... 95

Abbildung 106: Einschätzung der Veränderung seit 2007 - Vergleich Veranstalter vs. LW / FW......... 96

Abbildung 107: Schulbildung - Vergleich nach Branche ............................................................. 97

Abbildung 108: Gewählte Bildungsanbieter - Vergleich nach Branche ........................................... 98

Abbildung 109: Informationsquellen zu den Bildungsangeboten - Vergleich nach Branche .............. 98

Abbildung 110a: Bewertung des Bildungsangebotes (absolut) - Vergleich nach Branche.................. 99

Abbildung 110b: Bewertung des Bildungsangebotes (in \%) - Vergleich nach Branche .................... 100

Abbildung 111: Auswahlkriterien - Vergleich nach Branche .................................................... 101

Abbildung 112: Besuchte Kurse - Vergleich nach Branche ...................................................... 102

Abbildung 113: Lernformen - Vergleich nach Branche............................................................. 103

Abbildung 114: Empfundener Nutzen der Bildungsangebote - Vergleich nach Branche .................. 104

Abbildung 115: Veränderungen seit 2007 - Vergleich nach Branche ............................................. 104 


\section{ANHANG III - LISTE ALLER DOKUMENTE}

\begin{tabular}{|c|c|c|c|}
\hline & Titel & Herausgeber; Jahr; Ort & Dateiname \\
\hline [I] & & & B1 - Beschreibung des Veranstalters.doc \\
\hline [2] & & & $\begin{array}{l}\text { B2 - Beschreibung des Bildungsvorha- } \\
\text { bens.doc }\end{array}$ \\
\hline [3] & & & $\begin{array}{l}\text { B3-a \& B4b \& LSB - Bildung M331b\&d } \\
\text { Antrag \& Evaluierung NEU 09.xls }\end{array}$ \\
\hline [4] & & & Datenschutzverpflichtungserklärung.doc \\
\hline [5] & & & $\begin{array}{l}\text { Pruefproto- } \\
\text { koll UZAB ÖKL Prüfung 17122010.xls }\end{array}$ \\
\hline [6] & & BMLFUW; 2012; Wien & Projektauswahlkriterien für Bildung.doc \\
\hline [7] & & FAST Ort; & Kursfragebogen FAST Ort.doc \\
\hline [8] & $\begin{array}{l}\text { 26.KEBÖ-Statistik (2010 oder Arbeitsjahr 2009/10) An- } \\
\text { zahl der Teilnahmen }\end{array}$ & & image 002.png \\
\hline [9] & $\begin{array}{l}\text { Abschlussbericht Förderantrag OEBI-F1-008/10, Forstli- } \\
\text { che Aus- und Weiterbildung M } 111\end{array}$ & $\begin{array}{l}\text { Bundesforschungs- und Ausbildungszent- } \\
\text { rum für Wald, Naturgefahren und Land- } \\
\text { schaft; } 2011\end{array}$ & Abschlussbericht M111 008-10.doc \\
\hline$[10]$ & $\begin{array}{l}\text { Abschlussbericht Förderantrag OEBI-F1-009/10 (forstli- } \\
\text { che Nachwuchskräfte), Forstliche Aus- und Weiterbil- } \\
\text { dung M } 111\end{array}$ & $\begin{array}{l}\text { Bundesforschungs- und Ausbildungszent- } \\
\text { rum für Wald, Naturgefahren und Land- } \\
\text { schaft; } 2012\end{array}$ & $\begin{array}{l}\text { Abschlussbericht M111 forstl. Nach- } \\
\text { wuchs.doc }\end{array}$ \\
\hline$[11]$ & $\begin{array}{l}\text { Abschlussbericht Förderantrag OEBI-F1-012/11, Forstli- } \\
\text { che Aus- und Weiterbildung M } 111\end{array}$ & $\begin{array}{l}\text { Bundesforschungs- und Ausbildungszent- } \\
\text { rum für Wald, Naturgefahren und Land- } \\
\text { schaft; } 2012\end{array}$ & Abschlussbericht M111 008-10 1.doc \\
\hline$[12]$ & $\begin{array}{l}\text { Abschlussbericht Förderantrag OEBI-F1-013/11 (forstli- } \\
\text { che Nachwuchskräfte), Forstliche Aus- und Weiterbil- } \\
\text { dung M } 111\end{array}$ & $\begin{array}{l}\text { Bundesforschungs- und Ausbildungszent- } \\
\text { rum für Wald, Naturgefahren und Land- } \\
\text { schaft; } 2011\end{array}$ & $\begin{array}{l}\text { Abschlussbericht M111 forstl. Nach- } \\
\text { wuchs. doc 1.doc }\end{array}$ \\
\hline$[13]$ & Abschlussbericht Förderantrag OEBI-F3-012/10, Forstli- & Bundesforschungs- und Ausbildungszent- & Abschlussbericht M331 010-09.doc \\
\hline
\end{tabular}




\begin{tabular}{|c|c|c|c|}
\hline & Titel & Herausgeber; Jahr; Ort & Dateiname \\
\hline & che Aus- und Weiterbildung M 331 & $\begin{array}{l}\text { rum für Wald, Naturgefahren und Land- } \\
\text { schaft; } 2011\end{array}$ & \\
\hline$[14]$ & $\begin{array}{l}\text { Abschlussbericht Förderantrag OEBI-F3-020/11, Forstli- } \\
\text { che Aus- und Weiterbildung M } 331\end{array}$ & $\begin{array}{l}\text { Bundesforschungs- und Ausbildungszent- } \\
\text { rum für Wald, Naturgefahren und Land- } \\
\text { schaft; } 2012\end{array}$ & $\begin{array}{l}\text { Abschlussbericht M331 } 010-091 . d o c \\
\text { 1.doc }\end{array}$ \\
\hline$[15]$ & $\begin{array}{l}\text { Akkreditierung der System CERT ZertifizierungsgmbH zur } \\
\text { Zertifizierung von Personen (3.4.2009) }\end{array}$ & BMWFJ; 2009; Wien & str 2_BGB.pdf \\
\hline$[16]$ & Antrag auf Fördermittel & & Bildung Veranstalter.xls \\
\hline$[17]$ & $\begin{array}{l}\text { Antrag auf Fördermittel zur Förderung eines Vorhabens } \\
\text { entsprechend den „sonstigen Maßnahmen“ des Öster- } \\
\text { reichischen Programms für die Entwicklung des Ländli- } \\
\text { chen Raumes 2007-2013 }\end{array}$ & ARGE Huhn \& Co; 2009; Schlierbach & OEBI-L1-063_09 Geflügelwirtschaft III.pdf \\
\hline$[18]$ & Bericht des Rechnungshofes Reihe BUND 2012/7 & Rechnungshof; 2012; Wien & Rechnungshof_Leader.pdf \\
\hline$[19]$ & Bericht Weiterbildungssaison 2011/2012 & ARGE Huhn \& Co; 2012; Schlierbach & \\
\hline$[20]$ & $\begin{array}{l}\text { Beurteilungsbogen für die Sonderkategorie „Kooperati- } \\
\text { on“ }\end{array}$ & 2012 & \\
\hline$[2 \mid]$ & $\begin{array}{l}\text { Bewilligungscheckliste zum Antrag auf Fördermittel } \\
\text { Maßnahme 111/331 - Bildung Veranstalterförderung }\end{array}$ & BMLFUW; 2009; Wien & Pruefliste M 111 -OEBI-L1-063-09.pdf \\
\hline$[22]$ & Checkliste zur Auswahl von externen Kurstandorten & & $\begin{array}{l}\text { UZAB-Checkliste_Auswahl- } \\
\text { Kursstandorte_2010-12.doc }\end{array}$ \\
\hline$[23]$ & Endbericht M 111 Projekt & ARGE Huhn \& Co; 2011 & Endbericht 2.9.2011.pdf \\
\hline$[24]$ & $\begin{array}{l}\text { Evaluierung Berufsbildung: Halbzeitevaluierung der } \\
\text { Maßnahme Berufsbildung des ländlichen Entwicklungs- } \\
\text { programms }\end{array}$ & $\begin{array}{l}\text { AWI - Bundesanstalt für Agrarwirtschaft; } \\
\text { 2004; Wien }\end{array}$ & \\
\hline$[25]$ & Fachtrainer/in Zertifikat von SystemCERT & $\begin{array}{l}\text { SystemCERT, Zertifizierungsgesellschaft; } \\
\text { 2010; Leoben }\end{array}$ & str 1_Zertifikat Strohmaier.pdf \\
\hline [26] & Feedbackbogen LFI Österreich & LFI Österreich; 2009 & Feedback Bildungsmanagement leer.docx \\
\hline$[27]$ & Feedbackbogen LFI Österreich & LFI Österreich; 2010; Wien & $\begin{array}{l}\text { LFI Feedbackbogen Rev. 02-2010 vom } \\
\text { 3.8.2010.doc }\end{array}$ \\
\hline [28] & Foto von Audit 2010 & & Audit2010 010.jpg \\
\hline
\end{tabular}




\begin{tabular}{|c|c|c|c|}
\hline & Titel & Herausgeber; Jahr; Ort & Dateiname \\
\hline [29] & Genehmigung des Antrages aus der Maßnahme 111 & BMLFUW; 2009; Wien & Genehmigungsschreiben.pdf \\
\hline$[30]$ & $\begin{array}{l}\text { Kernaussagen des Projekts Wirkungsforschung für die } \\
\text { agrarische Bildung und Beratung }\end{array}$ & $\begin{array}{l}\text { Österreichisches Institut für Erwachse- } \\
\text { nenbildung (OIEB), Hochschule für Agrar- } \\
\text { und Umweltpädagogik; } 2012\end{array}$ & $\begin{array}{l}\text { Wirkungsforschung_BMLFUW_17 } \\
\text { 2012_Handout.pdf }\end{array}$ \\
\hline$[3 \mid]$ & $\begin{array}{l}\text { Kursprogramm 2012; Aus- und Weiterbildung an den } \\
\text { Forstlichen Ausbildungsstätten Ort und Ossiach }\end{array}$ & $\begin{array}{l}\text { Bundesforschungs- und Ausbildungszent- } \\
\text { rum für Wald, Naturgefahren und Land- } \\
\text { schaft; 2012; Wien }\end{array}$ & Kursprogramm12_Vers111108.pdf \\
\hline [32] & LFI Jahresbericht 2011 & LFI Österreich; 2011; Wien & LFI Jahresbericht 2011 Kern_final.pdf \\
\hline$[33]$ & Maßnahme 111 - Evaluierungsbericht 2010 & 2010 & M_111.pdf \\
\hline [34] & Maßnahme 111 - Evaluierungsbericht 2010 & 2010 & M_111korrekturen.doc \\
\hline [35] & Maßnahme 331 - Evaluierungsbericht 2010 & 2010 & M_331.pdf \\
\hline [36] & $\begin{array}{l}\text { Mein Betrieb - Meine Zukunft, Frühjahrstagung LK Vor- } \\
\text { arlberg }\end{array}$ & BMLFUW; 2012; Wien & $\begin{array}{l}\text { Paller_MB-BZ_15.03.2012_Hohenems } \\
\text { (1).pdf }\end{array}$ \\
\hline$[37]$ & $\begin{array}{l}\text { Mein Betrieb - Meine Zukunft, Monitoring-Bericht für } \\
\text { 2011/ } 2012\end{array}$ & & $\begin{array}{l}\text { TOP 2__Monitoringbericht_MB- } \\
\text { MZ_2011_2012.pdf }\end{array}$ \\
\hline [38] & $\begin{array}{l}\text { Österr. Umweltzeichen - Erläuterungen zur Richtlinie } \\
\text { UZAB Außerschulische Bildungseinrichtungen }\end{array}$ & BMLFUW; 2010; Wien & $\begin{array}{l}\text { UZÄB-Kriterien- } \\
\text { ERLÄUTERUNGEN_2011.pdf }\end{array}$ \\
\hline [39] & $\begin{array}{l}\text { Österr. Umweltzeichen Richtlinie UZAB; Außerschulische } \\
\text { Bildungseinrichtungen }\end{array}$ & BMLFUW; 2010; Wien & $\begin{array}{l}\text { UZAB_Bildungseinrichtungen- } \\
\text { Richtlinie_R2a-v3a_2010.pdf }\end{array}$ \\
\hline [40] & $\begin{array}{l}\text { Österreichisches Programm für die } \begin{array}{l}\text { Entwicklung des } \\
\text { Ländlichen Raums 2007-2013; Fassung nach } \\
\text { 5.Programmänderung }\end{array} \\
\end{array}$ & BMLFUW, 2011; Wien & \begin{tabular}{|l|} 
Fas- \\
sung_nach_5.Programmänderung_20102 \\
011.pdf
\end{tabular} \\
\hline$[4 \mid]$ & $\begin{array}{l}\text { Österreichisches Programm für die Entwicklung des } \\
\text { Ländlichen Raums 2007-2013, Fassung nach 6. Pro- } \\
\text { grammänderung }\end{array}$ & BMLFUW; 2012; Wien & Programm_V7.pdf \\
\hline [42] & $\begin{array}{l}\text { Österreichisches Umweltzeichen - Prüfbericht Außer- } \\
\text { schulische Bildungseinrichtungen }\end{array}$ & & UZAB-Prüfbericht ÖKL 2010.doc \\
\hline [43] & $\begin{array}{l}\text { Österreichisches Umweltzeichen für Außerschulische } \\
\text { Bildungseinrichtungen }\end{array}$ & BMLFUW; 2012; Wien & $\begin{array}{l}\text { uzab_k2a- } \\
\text { v2_bildungseinrichtungen_2012_folder_ } \\
\text { a5.pdf }\end{array}$ \\
\hline [44] & Positionspapier der Land- und forstwirtschaftlichen & Land- und forstwirtschaftliche Bundes- & \\
\hline
\end{tabular}




\begin{tabular}{|c|c|c|c|}
\hline & Titel & Herausgeber; Jahr; Ort & Dateiname \\
\hline & $\begin{array}{l}\text { Bundes-Lehrlings- und Fachausbildungsstelle für die } \\
\text { Verhandlungen zur Ländlichen Entwicklung 2014-2020 }\end{array}$ & $\begin{array}{l}\text { Lehrlings- und Fachausbildungsstelle; } \\
\text { 2012; Salzburg }\end{array}$ & \\
\hline [45] & Presseaussendung & Landjugend Österreich; 2011; Wien & PA_ISO-Zertifizierung_2011.doc \\
\hline [46] & Pressemitteilung & $\begin{array}{l}\text { Bundesforschungs- und Ausbildungszent- } \\
\text { rum für Wald, Naturgefahren und Land- } \\
\text { schaft; 2010; Gmunden und Ossiach }\end{array}$ & $\begin{array}{l}\text { PA_ISO- } \\
\text { Zertifizierung_Ausbildungsstaetten.doc }\end{array}$ \\
\hline [47] & Projektbericht Schule am Bauernhof 2010/2011 & LFI Österreich; 2012 & \\
\hline [48] & Qualitätshandbuch der LFI-Bundesgeschäftsstelle & LFI Österreich; 2012; Wien & Qualitätshandbuch LFI.pdf \\
\hline [49] & Qualitätsmanagement Ländliches Fortbildungsinstitut & LFI Österreich, 2012 & Bericht QM des LFI Juli 2012.docx \\
\hline [50] & $\begin{array}{l}\text { Rural Development Programme for Sweden - the period } \\
\text { 2007-2013 }\end{array}$ & $\begin{array}{l}\text { The Swedish Ministry of Agriculture; 2008; } \\
\text { Stockholm }\end{array}$ & \\
\hline$[51]$ & $\begin{array}{l}\text { Schule am Bauernhof in Oberösterreich; Auswertung der } \\
\text { Rückmeldungen absolvierter Bauernhofbesuche von } \\
\text { Lehrerinnen und Lehrern }\end{array}$ & 2010 & \\
\hline [52] & $\begin{array}{l}\text { Schule am Bauernhof in Oberösterreich; Auswertung der } \\
\text { Rückmeldungen absolvierter Bauernhofbesuche }\end{array}$ & 2011 & \\
\hline [53] & $\begin{array}{l}\text { Schule am Bauernhof in Oberösterreich; Auswertung der } \\
\text { Rückmeldungen absolvierter Bauernhoftage von Lehre- } \\
\text { rinnen und Lehrern aus dem Jahr } 2008 \text { und Empfehlun- } \\
\text { gen für die weitere Gestaltung der Programme auf den } \\
\text { Betrieben }\end{array}$ & $\begin{array}{l}\text { Hochschule für Agrar- und Umweltpäda- } \\
\text { gogik, Bachelorarbeit Thomas Neudorfer; } \\
\text { 2010; Wien }\end{array}$ & \\
\hline [54] & $\begin{array}{l}\text { Staatspreis für beispielhafte Waldwirtschaft; Beurtei- } \\
\text { lungsbogen für Betriebe mit beispielhafter Waldwirt- } \\
\text { schaft; Änderungsvorschlag } 6.11 .2012\end{array}$ & BMLFUW; 2012; Wien & \\
\hline [55] & $\begin{array}{l}\text { Statusbericht Priorität } 1 \text { - Wissenstransfer und Innovati- } \\
\text { on }\end{array}$ & BMLFUW; 2013 & Statusbericht PR 1.pdf \\
\hline [56] & $\begin{array}{l}\text { Strategische Schlussfolgerungen für Priorität } 1 \text { - Bildung } \\
\text { und Beratung }\end{array}$ & BMLFUW; 2012; Wien & $\begin{array}{l}\text { Strategische Schlussfolgerungen_PR 1- } \\
\text { Bildung und Beratung mit Matrixzuord- } \\
\text { nung.docx }\end{array}$ \\
\hline$[57]$ & $\begin{array}{l}\text { Strategische SWOT - Fragestellungen für die Strategie- } \\
\text { analyse - Priorität 1: „Innovation und Wissenstransfer“ }\end{array}$ & BMLFUW; 2012; Wien & $\begin{array}{l}\text { PR 1_Strategische SWOT Tableau Pr1 } \\
\text { Innovation_04122012.docx }\end{array}$ \\
\hline
\end{tabular}




\begin{tabular}{|c|c|c|c|}
\hline & Titel & Herausgeber; Jahr; Ort & Dateiname \\
\hline [58] & Technischer Zwischenbericht zur SWOT-Analyse & BMLFUW; 2013; Wien & Technischer Zwischenbericht_v3_0 \\
\hline [59] & $\begin{array}{l}\text { Verordnung (EG) Nr. 1698/2005 des Rates vom } \\
\text { 20.9.2005 über die Förderung der Entwicklung des länd- } \\
\text { lichen Raums durch den Europäischen Landwirtschafts- } \\
\text { fonds für die Entwicklung des ländlichen Raums (ELER) }\end{array}$ & Europäische Union; 2005; Brüssel & Verordnung des Rates.pdf \\
\hline [60] & Verpflichtungserklärung der Förderungswerber & & Verpflichtungserklärung-Beilage 09.pdf \\
\hline$[61]$ & $\begin{array}{l}\text { Vorschlag für eine VERORDNUNG DES EUROPÄISCHEN } \\
\text { PARLAMENTS UND DES RATES über die Förderung der } \\
\text { ländlichen Entwicklung durch den Europäischen } \\
\text { Landwirtschaftsfonds für die Entwicklung des ländlichen } \\
\text { Raums (ELER) }\end{array}$ & Europäische Kommission, 2011; Brüssel & VO-Vorschlag_LE2020.pdf \\
\hline [62] & Wirkungsforschung für das agrarische Bildungswesen & $\begin{array}{l}\text { Österreichisches Institut für Erwachse- } \\
\text { nenbildung (OIEB), Hochschule für Agrar- } \\
\text { und Umweltpädagogik; } 2012\end{array}$ & $\begin{array}{l}\text { Agrarpädagogische Hochschule Wir- } \\
\text { kungsforschung Bildung.pdf }\end{array}$ \\
\hline [63] & $\begin{array}{l}\text { Zertifikat der Quality Austria Trainings-, Zertifizierungs- } \\
\text { und Begutachtungs GmbH an nature adventure \& pro- } \\
\text { ject planning GmbH }\end{array}$ & $\begin{array}{l}\text { Quality Austria Trainings-, Zertifizierungs- } \\
\text { und Begutachtungs GmbH, 2011; Wien }\end{array}$ & Deutsch_ZER_schulewald.pdf \\
\hline [64] & $\begin{array}{l}\text { Zertifikat der Quality Austria Trainings-, Zertifizierungs- } \\
\text { und Begutachtungs GmbH an Landjugend Österreich }\end{array}$ & $\begin{array}{l}\text { Quality Austria Trainings-, Zertifizierungs- } \\
\text { und Begutachtungs GmbH, 2011; Wien }\end{array}$ & Landjugend ISO Zertifikat.pdf \\
\hline
\end{tabular}


Endbericht Bildungsevaluierung Ländliche Entwicklung LE 07-13 